\title{
Factors Influencing Provision of Enterprise Training: A Study of India's Information Technology (IT) Sector
}

by

Ashish Malik

\begin{abstract}
A thesis submitted to the
Victoria University of Wellington

in fulfilment of the requirements for the degree of

Doctor of Philosophy

in Human Resource Management and Industrial Relations
\end{abstract}

Victoria University of Wellington 


\begin{abstract}
Policy debates and academic research have emphasised the need for investment in human capital for improving national and organisational competitiveness and reducing unemployment. However, limited attempts have been undertaken to understand the factors that explain a firm's decision to invest in training. Most studies have been undertaken in the context of manufacturing firms in developed countries. Extant training demand models do not fully explicate the reasons for differences in training provision between firms operating in one industry sector. Further, the extant literature has often neglected a much cited need to consider, among other external factors, the influence of an organisation's clients on its decision to invest in training. Finally, little is known of the factors that influence a firm's decision to invest in training in the context of born-global, high-technology firms in a developing country context. This thesis is an attempt to bridge the above gaps. More specifically, it addresses how various factors-internal and external to the firm-interact with each other in shaping the final provision of training. Further, the thesis explores the reasons for variations in training between firms.
\end{abstract}

Owing to the still evolving state of theory and a relatively unexplored contextual setting, case study research is considered an appropriate method for this study. This thesis examines factors influencing training in ten organisations in India's IT services sector. Findings from case analysis suggest that the nature and extent of training is a result of complex interaction between an organisation's internal and external environment. An organisation's competitive strategy, the service markets it caters to, clients' specifications, workplace change, employee turnover, the temporal dimension of a process/project, and process complexity are found to be important factors in training decision-making. Contrary to the established view of training as an integral part of a firm's human resource management infrastructure, this thesis finds support for strong linkages between a firm's training infrastructure and its operations management. Further, this thesis reveals the critical and unexplored link between an organisation's quality management systems and its human resource management, as well as its learning and market orientation capabilities, in shaping the nature and extent of training. Findings from the study are then used in the development of a conceptual framework for understanding training decisionmaking in dynamic and high-growth outsourcing environments. Finally, areas for future research are identified. 
Dedicated to my father, the late Shri Rohit Kumar Malik

(26.2.1934-11.11.1991) 


\section{ACKNOWLEDGEMENTS}

It is difficult for me to encapsulate in this short acknowledgement the names of all of the people who have inspired me to complete my doctorate. Nevertheless, and in line with Hindu mythology, I would first like to thank my mother, Asha Malik. Secondly, I would like to express my deepest gratitude to my gurus (and supervisors), Dr Stephen B Blumenfeld and Professor George Lafferty. Without their support, motivation, and guidance at various stages of my learning, this thesis would not have reached its fruition. Their intellectual knowledge of theory and the research process made my learning experience memorable.

I would also like to thank all the case organisations for extending their willingness to participate in this research and providing support in data collection and feedback. I am indebted to my employer, The Open Polytechnic of New Zealand, for allowing me the research and development time and financial assistance for this study. I am also grateful to financial support received for this research from Victoria University.

This acknowledgement would be incomplete without my referring to the support and inspiration of my families of Nitish and Manish Malik, and Ved and Neelam Duggal, and of friends Pravir, Sandeep, and Srikanth. A very fond and special acknowledgement is due to my wife Namita, and my sons Madhav and Dhruv, for their patience, love, and sacrifices during this period of my learning. 


\section{TABLE OF CONTENTS}

$\begin{array}{rrr}\text { I. } & \text { Abstract } & \text { ii } \\ \text { II. } & \text { Acknowledgements } & \text { vii } \\ \text { III. } & \text { List of Figures } & \text { xi } \\ \text { IV. } & \text { List of Tables } & \text { xiii }\end{array}$

\section{CHAPTERS}

$\begin{array}{lr}\text { 1. Introduction } & 1\end{array}$

2. The IT Sector and its Contextual Environment 10

3. Literature Review 30

$\begin{array}{ll}\text { 4. Methodology } & 78\end{array}$

5. Within-Case Analyses $\quad 99$

$\begin{array}{ll}\text { 5.1. Organisation A } & 101\end{array}$

5.2. Organisation B 122

5.3. Organisation C 136

5.4. Organisation D 153

5.5. Organisation E 166

5.6. Organisation F 180

5.7. Organisation G 196

5.8. Organisation H 209

5.9. Organisation I 223

5.10. Organisation J 235

6. Cross-Case Analysis 251

$\begin{array}{ll}\text { 7. Discussion } & 284\end{array}$

$\begin{array}{lr}\text { 8. Conclusion } & 297\end{array}$

$\begin{array}{ll}\text { References } & 311\end{array}$

Appendices

Appendix-1 Information sheet 332

Appendix-2 Consent form 334

Appendix-3 Case study protocol 335

Appendix-4 Feedback request form 346

Appendix-5 Applying Six Sigma to a simple voice process 348 


\section{LIST OF FIGURES}

1. Growth of India's IT Sector 12

2. ITSS \& ITeS/BPO industry verticals

3. The Waterfall Model: Software development lifecycle 14

4. Continuum of ITeS/BPO services 16

5. A systems approach to performance improvement through HRD 33

6. Theory framework for applied disciplines: Boundaries, contributing, core, useful, novel, and irrelevant components 35

7. Market characteristics, competitive dynamics, and HR strategy in services 50

8. The Hayton et al. (1996) Model

9. A framework for market-based organisational learning 67

10. Specific roles of case study research in the context of general method of theory building 82

11. List and criteria for selection of multiple cases 89

12. Typical data analysis sequence 95

13. Business growth across verticals $\quad 102$

14. Temporal dimension of training: Changing emphases 105

15. Temporal dimension of training: Changing emphases 126

16. Temporal dimension of training: Changing emphases 139

17. Ownership structure of Organisation D 154

18. Temporal dimension of training: Changing emphases 157

19. Temporal dimension of training: Changing emphases 170

20. Temporal dimension of training: Changing emphases 184

21. Temporal dimension of training: Changing emphases 200

22. Organisation H: services portfolio 210

23. Temporal dimension of training: Changing emphases 213

24. Temporal dimension of training: Changing emphases 227

25. Temporal dimension of training: Changing emphases 239

26. Competitive strategy and volume of training 262

27. Process or project complexity and volume of training 264

28. Recruitment strategy and volume of training 267

29. Market orientation and volume of training 270

30. Learning orientation and volume of training 271

31. Quality management systems and volume of training 273

32. Evolving training capabilities and human resource costs 277

33. Temporal dimension of process life cycle and training volume 279

34. A framework for understanding enterprise training in India's IT sector 287 


\section{LIST OF TABLES}

1. Geographical destinations $\quad 12$

2. IT industry sector-wise break-up 15

3. India's rising productivity for software revenue per employee 21

4a. Theoretical foundations of main factors influencing investment in training: Focus and level of analysis $\quad 74$

4b. Study's questions and its relationship to the research questions 76

$\begin{array}{ll}\text { 5. Key categories } & 100\end{array}$

6. Conceptually clustered matrix of factors 119

7. Conceptually clustered matrix of factors 134

8. Conceptually clustered matrix of factors 149

9. Conceptually clustered matrix of factors 163

10. Conceptually clustered matrix of factors 178

11. Conceptually clustered matrix of factors 194

12. Conceptually clustered matrix of factors 206

13. Conceptually clustered matrix of factors 220

14. Conceptually clustered matrix of factors 233

15. Conceptually clustered matrix of factors 247

16. Nature and extent of training 256

17. Factors influencing training 263

18. Comparative table of growth in employee numbers of case organisations 281

19. Key propositions and study's findings 300 


\section{CHAPTER 1}

\section{INTRODUCTION}

This chapter presents an overview of the thesis. It sets out the research objectives and briefly discusses the context, the methodology, and the mode of analysis. It also lays out the structure of the thesis.

Extant literature acknowledges the role training, learning, and skill development play in the development of the individual, organisational, and dynamic capabilities necessary for achieving competitive advantage (Lepak \& Snell, 2003; Pfeffer, 1994; Teece et al., 1990; Harrison \& Kessels, 2004). Recent studies suggest that, globally, close to $\$ 100$ billion per annum is invested in employee training (Ketter, 2006). Further, investment in training and education has been noted to enhance national competitiveness and minimise the risk of unemployment (ILO, 2001; OECD, 1994; Bishop, 1994; Felstead \& Green, 1995; Ashton \& Green, 1996). Although the extant literature is well developed on the supply side of skills focusing on a nation's training and education infrastructure, the debate over the relative demand for the skills created by the educational infrastructure exists in policy, academic and business communities (Felstead et al, 2002; Keep \& Mayhew, 1996). The issue of the relevance of such skills needs to be understood in the context of where these skills are needed most - the organisations. Improvements at a national level can occur only in the context of improvement of the provision of training at an organisational level (Cappelli, 1994; Porter et al., 2004).

Most studies that explain training dynamics at an organisational level (Pettigrew et al., 1989; Dostie \& Montmarquette, 2007; Smith \& Hayton, 1999; Ridoutt et al., 2002; Smith et al., 2003, 2004) have been undertaken in a developed country context, focusing on manufacturing sector firms. With regard to developing countries such as India, and its services sector, especially its fast-growing IT services sector, little research has been undertaken on investment in training at an organisational level, despite an increasing gap between the demand for, and supply of, a skilled pool of human capital (Narayanan \& Neethi, 2005; NTITSD, 2003; NASSCOM-KPMG, 2004). Recent estimates from India's IT and IT-enabled services (ITeS) sectors suggest that US\$1.5-2.0 billion per annum was spent on training in 2007, with the top four players accounting for close to US\$500 
million of the total amount spent, an average training budget of about US\$5000 per person (Economic Times, 2007). This thesis examines the factors that drive a firm's decision to invest in training in India's information technology (IT) sector. This study also seeks to explain why some firms invest more in training than other firms in the same industry sector.

The study is situated within the wider literature on human resource development (HRD), and focuses on a much-cited and often neglected need to study the external factors, especially the influence of an organisation's customers/clients, in a firm's decision to train (Leimbach \& Baldwin, 1997; Bing et al., 2003; Short, 2006). External factors are particularly important given the distinctive features of the IT sector, such as the high level of interdependence between service providers and client firms, shorter project/process life cycles, and the dynamic nature of the coordination that exists between client and provider firms (Banerjee, 2004, 2006). In addition to extending the extant theory-building attempts in the field of enterprise training (Pettigrew et al., 1988; Dostie \& Montmarquette, 2007; Smith \& Hayton, 1999; Ridoutt et al., 2002; Smith et al., 2003, 2004), this study's distinctive contribution is in identifying the influence of market orientation, learning orientation and quality management systems on the nature and extent of training in a dynamic outsourcing environment, in the context of India's IT sector. Before the study's research problem and contributions are explicated, the following section provides a definition of training for the purposes of this study.

\section{Defining Training}

The study and practice of HRD focuses mainly on issues relating to training and development, and career and organisation development (McMaglan, 1989; De Cieri \& Holland, 2006; Swanson \& Holton, 2001). This stream of literature has witnessed attempts at HRD conceptualisation and theory building (Barrie \& Pace, 1998; Kuchinke, 1998; Lee, 2001; Mankin, 2001; McLean, 1998, 1999; McLean \& McLean, 2001; Sambrook \& Stewart, 1998; Swanson, 2001). A definition of HRD that has received significant consensus is that it is "a process for developing and unleashing human expertise through organisation development and personnel training and development for the purpose of improving performance" (Swanson \& Holton, 2001, p. 4).

Despite this definition and given the central focus training has within the field of HRD, there are varying academic and practitioner definitions of training, development, 
education and learning (Garavan, 1997). The Cambridge Dictionary defines training as the process of learning the skills you need to do a particular job or activity. Generally, within the human resource management (HRM) and HRD literatures, training is defined as a planned and systematic effort to modify knowledge, skills and attitudes through learning experiences, to achieve effective performance in an activity or a range of activities (Garavan et al., 2000). Garavan (1997) argues that one should "view the concepts of training, development and education as an integrated whole with the concept of learning as the glue which holds them together" (p. 40). In a major research project on enterprise training in Australia, Hayton et al. (1996) considered enterprise training as "all forms of skill formation activity relevant to the operation of the enterprise. This includes formal and informal training, and on-site and off-site education and training" (Hayton et al., as cited in Ridoutt et al., 2002, p. 27).

While most training definitions are focused on individuals and their performance on the job, for the purposes of this thesis the definition provided by Hayton et al (1996) is modified. Training is broadly conceptualised, in the context of organisations operating for profit, as: all learning activities relevant to the operation of an enterprise and includes formal and informal training, development, and education provided by the organisation, internally or externally. Further description of the dimensions of the nature and extent of training considered in this study is offered in Chapter 3.

\section{The Problem in Context}

The research problem in this study is grounded at two levels: a theoretical level and a practitioner level. In the 2003 special issue, Advances in Developing Human Resources (the Academy of HRD's flagship journal for theory advancement issues) - identified some key challenges faced by the field of HRD and set forth a new agenda for researchers. Among a range of issues identified, the special issue pointed towards the need for an organisation to move away from an inward-looking approach, serving a narrow set of stakeholders, usually as its shareholders, to an outward looking approach, serving the needs of a wider group of stakeholders, such as its customers (Bing et al., 2003; May et al., 2003; Rouna et al., 2003; Packar \& Sharar, 2003). The need to also consider what customers or clients need from HRD has been identified in the past (Leimbach \& Baldwin, 1997) and even recently noted as a "gap between research and practice" following a 10-year review of HRD literature (Short, 2006). Although some 
authors have advanced the key underlying theories of HRD (Swanson, 2001; Mankin, 2001) and have outlined the main dependent variables or outcomes for HRD (Swanson, 1999; Swanson \& Holton, 2005), the issue of adopting an outward-looking approach, and of undertaking research that delineates the training drivers (an approach that precedes any intended outcomes of HRD) is largely unaddressed.

For an understanding of the problem at a theoretical level, firstly, a deeper analysis and explanation of the phenomenon is needed on what drives or triggers investment in training. This analysis should precede the discussion on the why or outcomes/purpose of HRD (for example, performance improvement, productivity enhancement, individual learning). Although there have been some attempts to delineate and model the factors that appear to drive or trigger investment in training (OECD/CERI, 1988; Pettigrew et al., 1988; Green et al., 2003; Dostie \& Montmarquette, 2007; Smith \& Hayton, 1999; Smith et al., 2003, 2004;), the research generally lacks theoretical elegance and articulation to HRD and its related theories. With the exception of Smith and Dowling (2001) and Ashton and Sung (2006), the above studies also tended to adopt an input-output model of certain factors that drive firms' investment in training. Although the above models offer us a framework and some understanding of the relationships between key explanatory factors, the studies offer limited understanding of why one factor does not explain the variance in another industry or even within the same industry type.

Secondly, existing research has predominantly been undertaken in the context of developed economies, such as those of the UK, USA, Australia, Canada, New Zealand and other OECD countries. In the context of developing countries, such as India, limited literature is available about training in general, and its drivers in particular. Further, the main purpose of the above studies was usually to investigate and possibly address the governments' socio-economic policy needs relating to education and training. For example, UK studies looked at why national skills and training systems failed (Finegold \& Soskice, 1988). Australian studies sought to clarify or demystify the low level of training provision by firms (Smith \& Hayton, 1999; McIntyre et al., 1996). Following a holistic approach to developing human capability in New Zealand workplaces, researchers from New Zealand (Bryson \& O’Neil, 2008) examined the drivers and barriers at institutional, organisational and individual levels. Academics and practitioners in developing countries have to rely almost exclusively on research disseminated by North American and European HRD journals. Further, as such research tends to be from 
developed countries, contextual factors, which are gaining importance, remain largely unaddressed in a developing country context (Woodall, 2005). Also, given that HRD is an applied discipline, this research thesis will utilise Swanson's (2007) work for building a theoretical framework in applied disciplines such as HRD. To this end, this thesis will consider HRD's relevant theoretical foundations, as well as include any novel theories that best explain the phenomenon under study (Swanson, 2007).

Thirdly, for firms to adopt an outward-looking approach, it is important that they look at the needs of their external clients or customers (Leimbach \& Baldwin, 1997; Bing et al., 2003; Short, 2006). In the context of research in HRD, it is important not only because of limited literature on the topic, but also because of the added value that might emerge from an organisation's clients or customers, given the richness and reach of information, as well as the influence clients/customers now have on an organisation's products and services. Added value could occur through customer feedback about product/process improvements and ideas for new product development. Such feedback loops are noted in the service, high technology, and consumer goods sectors (Baker \& Sinkula, 2002, 2005). Further, staying close to customers/clients presents HRD practitioners and academics with an opportunity to engage in evidence-based HRD practice (Hamlin, 2007) and to address the academic discourse about the disconnection between HRD theory and practice (Holton, 2004; Yorks, 2005).

Given that interaction between service providers and clients/customers is highest in the services sector, and especially in the high technology areas, where clients and service organisations demonstrate dynamic coordination and collaboration to enable service delivery (Banerjee, 2004), this research project focuses on one of India's services sectors, its IT sector. Recent research points to the limited studies on HRM and skills development issues of IT professionals (Githens et al., 2008; Kaplan \& Lerouge, 2007). Further reasons for choosing India's IT sector are discussed in the following section.

First, within the services sector in India, its IT sector is relatively under-researched for HRD issues in general (Rao \& Abraham, 1986; Yadapadithaya, 2001) and for training drivers in particular. Second, the sector is posting dynamic growth rates in revenues and employment and is constantly undergoing technological change, thus requiring new research to inform theory-building and future practice needs for the sector and its subsectors. Third, the organisations vary greatly in the size and range of services offered, and 
there is likely to be more than a single explanation for investment in training. Typically, large IT firms secure long-term contracts in a range of industries and specialisations, whereas small and medium-sized firms attract short-term contracts in a narrow area of specialisation. Fourth, given the dynamic environment, short product/process lifecycles, and the constant need for knowledge acquisition, transfer, and absorption to and from clients, a study of the temporal dimension of the projects/process in relation to training is appropriate. Such diversity and dynamism in the industry presents an opportunity for HRD researchers-theorists to explore how best to synthesise or modify any applicable theoretical frameworks suitable for ensuring an outward-looking and market-based organisational learning approach (Sinkula et al., 1997; Baker \& Sinkula, 1999, 1999a, 2002, 2005). This framework will be explained later in the literature review chapter.

At a practitioner level, first, insights into training drivers and the reasons for them are important for HRD practitioners to effectively utilise and combine available resources with a view to engaging in evidence-based HRD (Hamlin, 2007). Second, given the uneven profile of India's IT sector (Heeks, 1998) and that the bulk of the revenues are accounted for by large firms, which constitute less than $1 \%$ of the total firms operating in the sector (Ethiraj et al., 2005), it is important for HRD practitioners to understand any differences in the level of training drivers in small, medium-sized and large firms, as well as those operating in different sub-sectors of the industry. Third, the sector's high employee turnover and growth in employment in the last two decades requires HR and HRD practitioners to gain a better understanding of how to deal with ongoing people management and HRD issues (NASSCOM, 2004).

Using a case study methodology, this research will analyse the factors that trigger investment in training in the context of India's information technology (IT) sector and provide detailed descriptions and analysis of how these factors interact with each other. Finally, after considering the what and how questions, it will analyse and interpret the contextual reasons why IT firms in India invest in training and offer explanations for variation in training between firms.

To this end, this study aims to:

- identify what the key factors are that drive a firm's decision to invest in training

- explain how an organisation's clients influence its decision to provide training 
- explain how various factors - internal and external-interact and influence the nature and extent of training

- explain why some firms invest more than others even when they are in the same industry.

\section{Research Contributions}

The research is significant for a number of reasons and the key contributions of the study can be summarised on the basis of theoretical, methodological, and practitioner contributions.

\section{Theoretical Contributions}

The research contribution is unique as it shows how firms in a dynamic and high-growth outsourcing environment within the Indian context use its strategic capabilities to respond to market forces. This study brings together previously ignored relationships between constructs from different disciplines in an understandable manner and, in the process, contributes to unexplored and critical dimensions relevant to the body of knowledge pertaining to training and HRD. In particular, the contributions are in the following three areas:

The high-technology services sector in the context of a developing country

Existing HRD literature in India mostly focuses on national HRD trends and the impact of India's liberalisation policies on firms' training and development infrastructures (Rao, 2004; Rao \& Abraham, 1986; Rao \& Varghese, 2009; Yadapadithaya, 2001). There is limited HRD research undertaken in the services sector and even less in the high technology sectors, such as the information technology (IT) sector. Further, recent studies on India's IT sector have concentrated only on the low added-value segment in customer service centres, such as call centres (Taylor \& Bain, 2004; Shah \& Jerrard, 2004). In general, research on call centres focuses on three main strands of literature. The first dominant strand comprises the organisation of work within a Taylorist paradigm and looks at issues related to monotonous work and excessive monitoring and control (Taylor \& Bain, 1999; Burgess \& Connell, 2004). The second strand includes employee voice, resistance, and other coping mechanisms (van den Broek, 2002). The third strand uses a unitary approach in dealing with labour turnover, employee commitment, service quality, training staff, strategic human resource management and business models, and managing stress (Batt et al., 2000; Carton et al., 2004; Fernie and Metcalf, 1998; Hutchinson et al., 
2001; Shah and Jerrard, 2004). This research project contributes to the limited body of research on training drivers in two sub-sectors: India's IT software services (ITSS) and IT-enabled services (ITeS) sub-sectors.

\section{Client or customer perspectives}

Owing to the increasing investment in employer-sponsored training (Economic Times, 2007; Ketter, 2006), shareholders, customers and clients ought to know whether such training is effective and the factors that influence training decision making. Further, given the huge amounts spent on training, HRD practitioners and academics have an obligation to consider the varying needs of a range of stakeholders (Bing et al., 2004; Chermack et al., 2003; Rouna et al., 2003), to consider a two-way customer-service model (Wang, 2003), and to look for theories that help to meet the varied needs of an organisation's stakeholders. To this end, this research contributes by considering training from the perspective of an organisation's clients, employing a market-based organisational learning framework (Sinkula et al., 1997; Baker \& Sinkula, 1999, 1999a).

\section{Temporal dimension}

Although some neo-human capital theorists (Woznaik, 1984, 1987; Bartel \& Lichtenberg, 1987) and learning theorists suggest a temporal dimension in any learning that organisations undergo, there is little known of the training decision-making that occurs at a project or process level. This thesis will fill this gap to help practitioners to plan for the volume and diversity of training for new projects and processes from a temporal dimension.

\section{Methodological Contribution}

In the context of a developing country such as India, this study's contribution is in analysing the findings from the viewpoint of key informants of the study in their natural settings. The relatively under-researched contextual setting and research topic, and the theory-building case-study research strategy adopted (Dooley, 2002; Yin, 2003; Eisenhardt, 1989) to study a dynamic outsourcing environment are unique contributions from a methodological viewpoint. Understanding the phenomenon in a natural setting enables richness and reach. Triangulation of data allows flexibility during various stages of data collection, analysis, and theory-building (Eisenhardt, 1989). 


\section{Practice Contribution}

Owing to the acknowledged role training plays in improving an organisation's performance at various levels, the explanations for variation in training for firms operating in different strategic milieus are critical to practitioners' understanding. The study offers deep insights into the training drivers and the complex decision making that surrounds it. Further, gaining an understanding of how certain organisational capabilities and human resource management practices in a dynamic outsourcing environment are developed will assist practitioners to make strategic choices in their training decision making.

\section{Conclusion}

In this chapter, an overview of the research, of the nature of the research problem, and of the gaps has been presented. In the next chapter, a contextual background of India's wider socio-economic and political environment is provided, to enable a better understanding of the research problem, given its unique cultural, social, economic and political environment and the unique stages of evolution of the IT sector. The rest of the thesis is organised as follows: Chapter 3 critically evaluates the theoretical perspectives from extant literature and identifies the specific research questions that follow. Chapter 4 presents the justification and details of the use of case-study methodology followed by this thesis. Chapter 5 presents the within-case analyses. Chapter 6 discusses themes emanating from cross-case analysis. Chapter 7 discusses the findings and develops a conceptual framework for understanding training decision-making in case organisations. Chapter 8 presents implications for practice, policy, and theory development. The thesis concludes by discussing the limitations of the current research and directions for future research. 


\section{CHAPTER 2}

\section{THE IT SECTOR AND ITS CONTEXTUAL ENVIRONMENT}

\section{Introduction}

This chapter begins with a brief overview of India's socio-economic composition and its demographic details, and then discusses the evolution of its IT sector in light of the global development and expansion of the IT sector. Rao (2004) argues that for framing a nation's HRD needs it is important to understand its human resource endowments, the structure of its economy, its culture, and its demographic characteristics. Understanding how the sector evolved and how it continues to demonstrate growth enables a better understanding of the contextual environment in which firms organise their training activity.

\section{India: Key Demographics}

India is the second most populous country and the largest democracy in the world. With an estimated population of 1.17 billion (World Bank, 2007), India is a country of 34 geographical units, 18 official languages, and numerous religious beliefs and social caste hierarchy systems (Rao, 2004). It is predominantly an agricultural economy, with a literacy rate of about $61 \%$ and about $29 \%$ of its people living below the poverty line (World Bank, 2007).

Socio-cultural beliefs in India reflect a strong orientation towards education: a typical middle-class family would desire their child to become a doctor or an engineer. With a workforce of close to 440 million people and a significant majority (close to 50\%) of its population less than 25 years of age (Rao \& Varghese, 2009), India also has one of the largest pools of technically qualified and English-speaking graduates, working in a range of industries. Although India has 338 universities and about 12,000 higher education colleges producing 2.5 million graduates each year in various disciplines, including about 150,000 engineering graduates (MHRD, 2007), the issue of their immediate 
employability in today's globalised economy is a real concern (NASSCOM-KPMG, 2004). Despite the fact that India boasts one of the oldest technical colleges in the world - the School of Survey in Chennai - set up in 1794, there is a need to increase the industry-academia collaboration to keep up with the growing demands of the IT sector (NASSCOM-KPMG, 2004; NTITSD, 2003).

\section{Economic Development}

Before the Government of India introduced its economic liberalisation programme of the 1990s, the dominant logic was import substitution and self-reliance. Although this led to well-developed primary, secondary and tertiary sectors, it also created the licence-Raj system, which regulated the number of industrialists and their outputs by issuing licenses and regulating quotas. During this period, the education infrastructure expanded to ensure a constant supply of skilled people for these industry sectors. Although most service and manufacturing sectors have been in existence for more than a century (for example, banking, finance, power, insurance, railways, and petroleum refineries), their growth gained further momentum immediately after India became a republic in 1947, and centralised planning took over to meet the twin national goals of import substitution and self-reliance. The result was the development of knowledge, experience and skills in a range of industry sectors and a well-developed labour market, ensuring a gradual but steady growth of industries. However, numerous deterrents to full scale economic development existed in the licence-Raj era, such as corruption, bureaucracy, "red-tapism" and the monopolistic participation of state-owned enterprises in key industry sectors. Foreign investment and ownership was both limited and regulated, leading to limited inflow of foreign exchange and technological knowledge.

When one considers the poor and backward country image that India has had and its protected and technologically underdeveloped economy, which has been on the brink of bankruptcy, the success of India's IT sector needs further exploration. It warrants even more exploration given the mixed bag of media responses about the growth of India's IT sector. The following section provides an overview of the nature and structure of India's IT sector. 


\section{Overview of India's IT Sector}

\section{Revenues, Employment, and Structure}

Although the IT industry started in the mid 1970s, it is only in the last two decades that the sector has witnessed a steady growth in revenue and employee numbers. The sector grew at an average compounded annual rate of 50\% between 1989 and 2000. Only recently has the annual growth rate stabilised to around 30\% per annum (NASSCOM, 2007). Its revenue has increased from US $\$ 175$ million in $1989-90$ to US\$47 billion in 2006-07 (see Figure 1), of which exports, primarily to the US and European markets, comprise $67 \%$ and $25 \%$ respectively of the total revenue (See Table 1). The sector contributes to $5.4 \%$ of India's GDP.

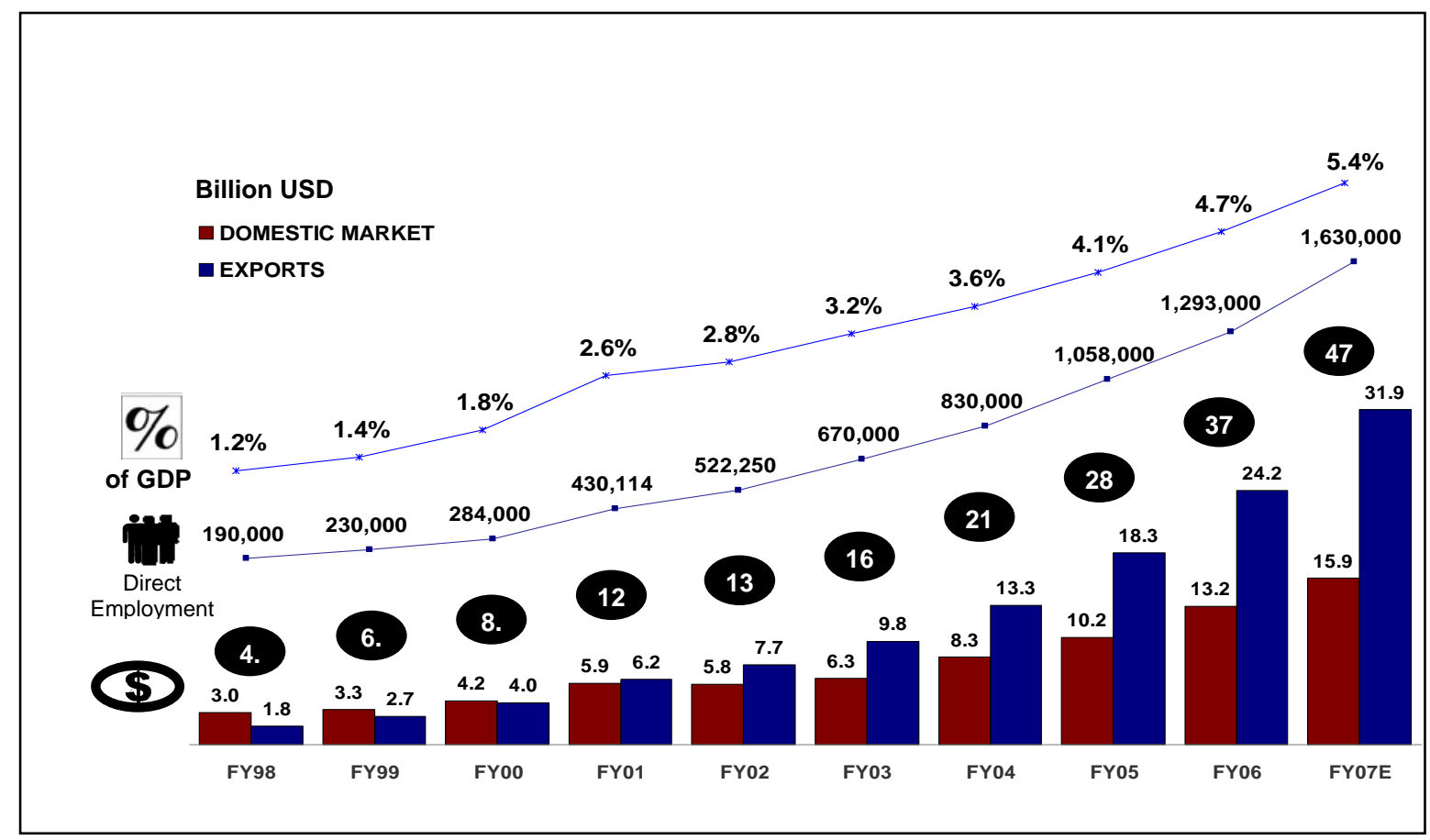

Figure 1. Growth of India's IT sector.

(NASSCOM, 2007)

Table 1

Geographical Destinations

\begin{tabular}{|l|l|l|l|}
\hline Market & $\mathbf{2 0 0 5}$ & $\mathbf{2 0 0 6}$ & $\mathbf{2 0 0 7}$ \\
\hline Americas & $68.30 \%$ & $67.18 \%$ & $67.18 \%$ \\
\hline Europe & $23.10 \%$ & $25.13 \%$ & $25.13 \%$ \\
\hline Rest of the World & $8.60 \%$ & $7.69 \%$ & $7.69 \%$ \\
\hline
\end{tabular}


Recent figures report revenue of US\$64 billion and an employment of 2.1 million for the year ending 2008. Broadly, the sector can be classified into three sub-sectors:

- IT hardware manufacturing (ITHM)

- IT software services (ITSS)

- IT-enabled services (ITeS), which includes business processes outsourcing (BPO) services.

Because the focus of this research is the services sector, the ITHM sub-sector will be excluded from this study. Within ITSS, there are numerous areas of specialisation and service lines. The sector caters to a range of industries (commonly called "verticals" in the sector), and within these industries it caters to a range of specialisations (commonly called "domains" or "horizontals" in the sector). Figure 2 provides a snapshot of the main industries the sector is catering to. BFSI here refers to Banking, Financial Services and Insurance. It is important to note here that these industries

Financial Year 2006

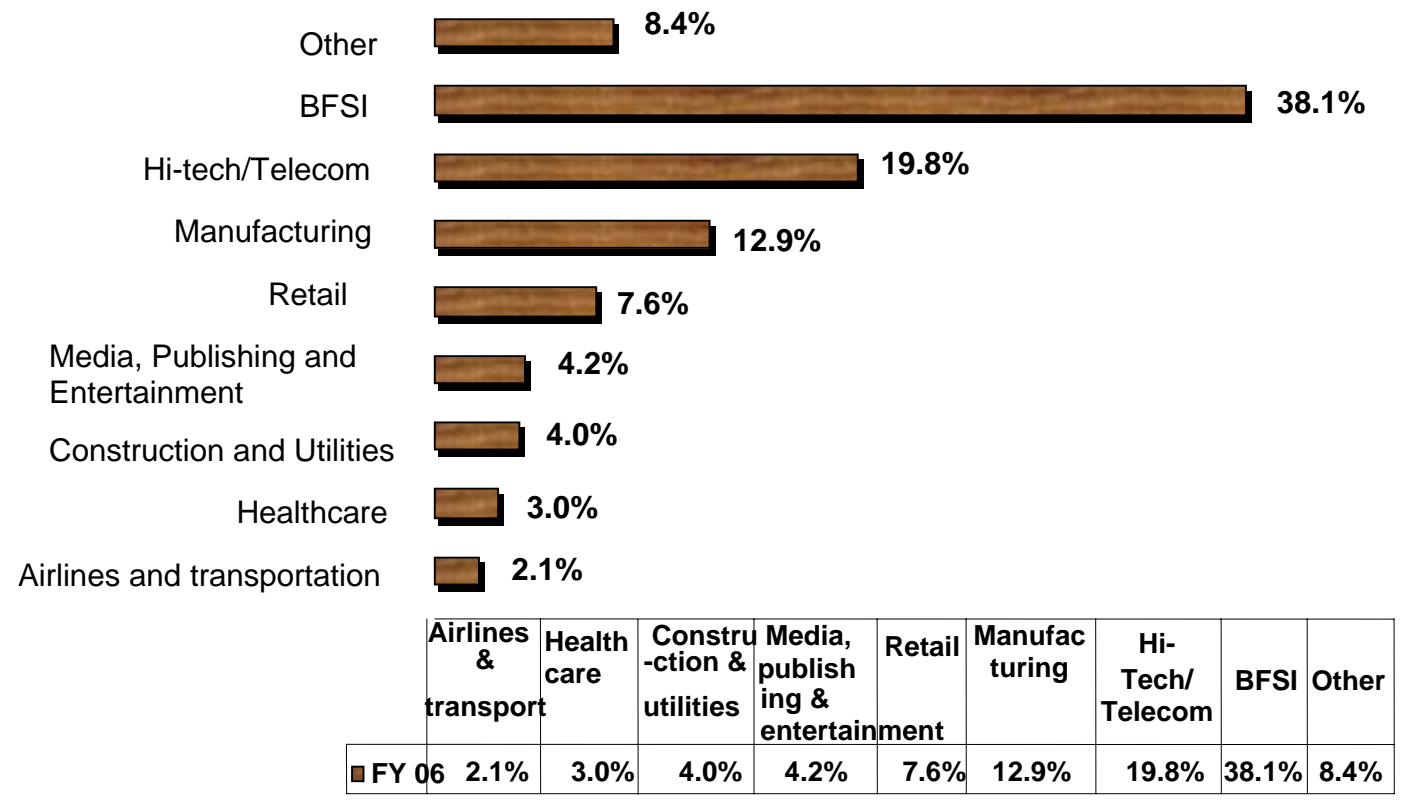

Figure 2. ITSS \& ITeS/BPO industry verticals.

(NASSCOM, 2007)

have also been in operation for a long time in India, some (for example, BFSI, Oil and Gas, Construction and Utilities, Education) for more than a century. This would suggest that a labour market in these sectors is well developed, both in terms of quantity and 
quality. In other words, there exists a labour market of skilled and experienced human resources to be tapped by the ITSS and ITeS/BPO sectors although they are not servicing the cutting edge of the high-end technology market.

The ITSS sector provides services that are typical of a software development life cycle; see, for example, Figure 3 below. The Waterfall Model of the life cycle of software development comprises a requirements analysis, higher and lower level design, coding, testing, and post-production maintenance and support. Other services in ITSS include IT consulting, infrastructure management, networking services, product development and embedded software design and development. Firms may provide services for the entire software development cycle, typical in a product development environment, or provide services for various stages, for example, coding, testing, application maintenance and support, typical of firms operating in a project environment.

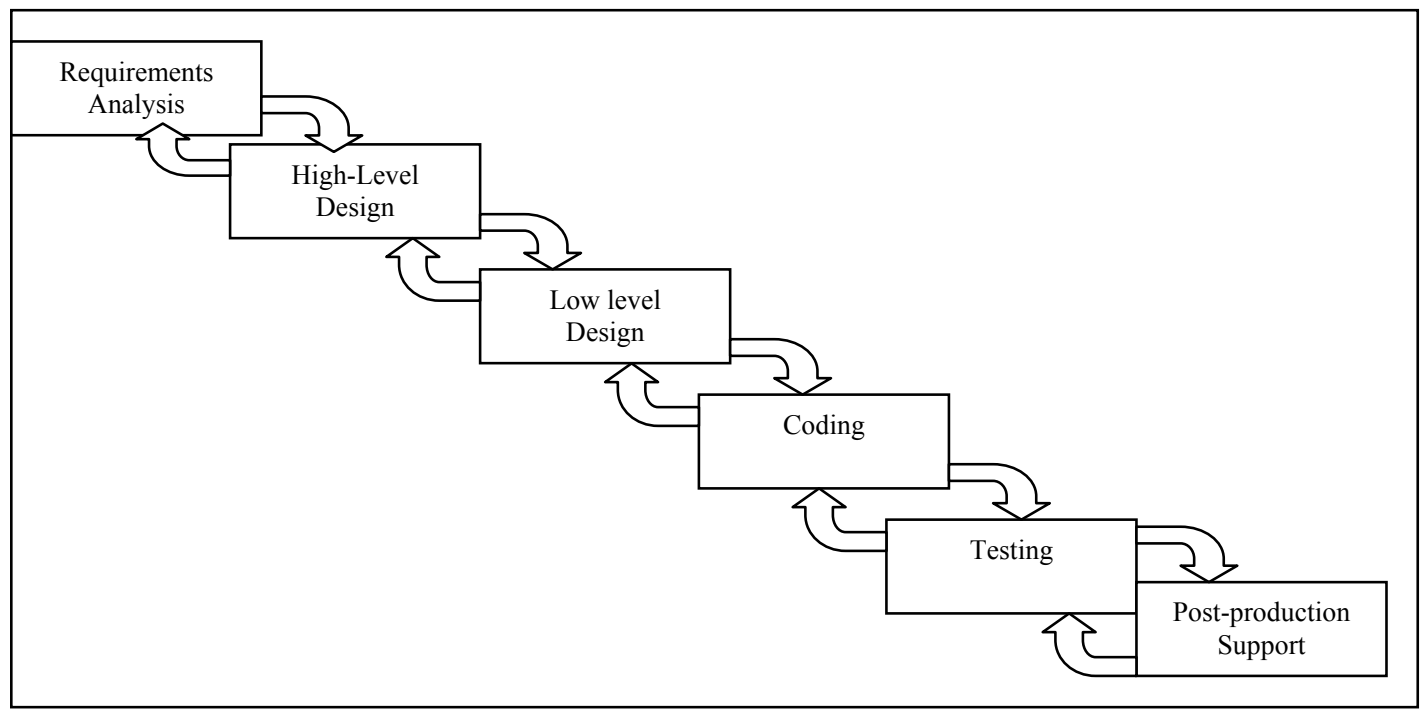

Figure 3. The Waterfall Model: Software development life cycle.

(Adapted from Arora et al., 1999)

In short, ITSS mainly includes firms operating in either a project environment, offering a range of services in the development cycle, or a product environment, or both. Typical software product firms develop and sell software packages, for example, Microsoft's MS Office or SAPs My-SAP-ERP. A closer examination of the sector reveals that the share of product development work relative to IT services (or projects) has always been extremely low. It was $5 \%$ in 1990 . Only recently has the product development and R\&D work increased: from about 8\% of software exports in 1999 (which was also the year that the government made changes to foreign ownership rules, intellectual property protection and 
venture capital (Dossani, 2005; Heeks, 1996, 1998)) to 27\% of ITSS exports in 2007. (Refer to Table 2 for Years 2006 to 2008.) Further examination of the revenues of key players in the sector reveals that less than $1 \%$ of all of the firms in the sector (about 3000) account for almost $60 \%$ of the export revenue (Athreye, 2005; Ethiraj et al., 2005; NASSCOM, 2006). This percentage would suggest that a small number of firms continued to grow at higher than the average growth rate posted by the sector and that there are differences in organisational performance between some of the larger firms and other firms in the sector.

\section{Table 2}

IT Industry: Sector-Wise Break-Up

\begin{tabular}{|c|c|c|c|}
\hline USD billion & FY 2006 & FY 2007 & FY 2008 P \\
\hline IT Services & 17.8 & 23.6 & $30-31$ \\
\hline Exports & 13.3 & 18.0 & - \\
\hline Domestic & 4.5 & 5.6 & - \\
\hline Eng Services and R\&D, S/W Prods & 5.3 & 6.5 & $\sim 8$ \\
\hline Exports & 4 & 4.9 & - \\
\hline Domestic & 1.3 & 1.6 & - \\
\hline ITES-BPO & 7.2 & 9.5 & $11-12$ \\
\hline Exports & 6.3 & 8.4 & - \\
\hline Domestic & 0.9 & 1.1 & - \\
\hline Total Software and Services Revenues & 30.3 & 39.6 & $49-50$ \\
\hline Of which, exports are & 23.6 & 31.4 & $39-40$ \\
\hline Domestic & 6.7 & 8.2 & $\sim 10$ \\
\hline Hardware & 7 & 8.2 & - \\
\hline Total IT Industry (including Hardware) & 37.4 & 47.8 & - \\
\hline
\end{tabular}

(NASSCOM, 2007)

A notable point here is that most of these firms rely on software services rather than software products as their main source of revenue, despite the popular belief that the development of a software brand/product is essential for sustained competitive advantage. While product superiority and leadership is noted for brands such as Microsoft, Oracle, Adobe and SAP, it has been argued that the services market is much larger than the 
product market (Banerjee, 2004). Product development requires higher level investment and capabilities in research and development, marketing and promotion, which most Indian firms did not possess in the early stages of their evolution. Banerjee (2004) argues that product superiority arises from a manufacturing mindset and thus the product will always be perceived as superior, whereas projects, although perceived as risky and less rewarding, offer some advantages over the product environment. For example, the same programming code can arguably be deployed over different projects. Projects require limited upfront investment in R\&D and marketing. While the revenue is generally much higher in product environments owing to global reach, their profitability is similar to that of firms in the IT services sector (Arora, 2006). Finally, even in a product environment, there will always be a need for project service firms to support, maintain, and undertake coding, testing, or even collaborative product development for product firms. This is the market space where the Indian software services firms have carved a niche for themselves, and they are gradually moving up the value chain. What is still to be seen is whether any Indian firms are able to develop and register significant patents for their products, although there are some early signs of confidence emerging (Arora, 2006).

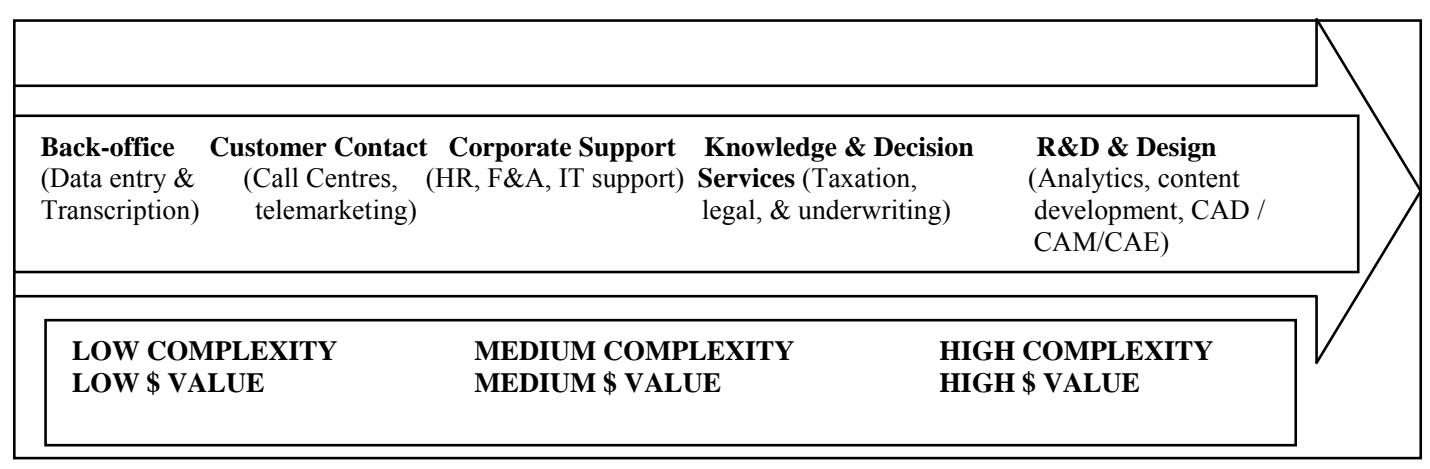

Figure 4. Continuum of ITeS/BPO services.

(Adapted from NASSCOM \& KPMG, 2004)

The ITeS/ BPO services sub-sector on the other hand can be broadly classified into voice (for example, customer care centres such as call centres) and non-voice services (for example, back-office business process outsourcing). The latter attracts a medium to high dollar rate as against the former. Examples of these high-end non-voice services include market analytics, market research, HR outsourcing and legal services. There is almost a price and added value continuum for this sub-sector (See Figure 4 above). 


\section{Business Models and Nature of Contracts}

India's IT sector today has a range of business models in operation. They can be traced back to its stages of evolution. Broadly, the firms in the sector include domestic firms, multinational corporations (MNCs), and some form of collaboration between the two. Firms operate as third-party service providers (most large domestic firms), captive operators (commonly referred to as wholly owned subsidiaries of MNCs) or joint venture business partnerships, for example, third-party service providers setting up a shop for MNCs on a build, operate and transfer (BOT), or build, operate, lease and transfer (BOLT), basis. The business contracts for third-party service providers were mainly "Time and Material" (T\&M) contracts in the early stages of their development, where projects were undertaken on the basis of billable time and materials (labour and infrastructure) supplied for clients' projects. While these contracts are still prevalent, improving organisational capabilities, changing environmental conditions, and the competencies of domestic firms have led to the emergence of fixed-price (FP) contracts (Athreye, 2005). For MNC captives, the business model is based on a simple cost plus a mark-up margin. For domestic firms, T\&M contracts were the norm. Because they lacked credibility to undertake FP projects successfully, the only way that the client firm could get a better deal through service providers was to negotiate a tough (low) dollar rate, to cover any time and material overruns.

\section{Global Growth of High-Tech Clusters and India's IT Sector}

The rapid growth of high-tech clusters around the world has attracted academic interest and produced a burgeoning body of literature offering explanations of the various factors that have led to their emergence (Arora et al, 2001a; Arora \& Gambardella, 2004; Arora \& Athereye, 2002; Bresnahan et al., 2001; Finegold, 1999; Saxenian, 1994, 2001). While the extant literature offers useful insights about the roles of human capital agglomeration, interdependence and cooperation, and their externality-inducing effects on clusters of high-tech firms, in the context of India's IT sector the explanations are not generalisable to a firm level for numerous reasons.

First, the literature offers a partial explanation as to why only one high-tech cluster - the IT sector-evolved and other high-tech sectors in India, such as the pharmaceuticals and engineering hardware sectors, did not perform well (Athreye, 2005; Subramaniam, 1992). 
Second, with reference to the geographical ecologists' model, Indian IT clusters weren't concentrated in any one city and did not follow a set pattern. For example, they started in Mumbai and then became established in New Delhi, Pune and finally Bangalore, which is now referred to as India's Silicon Valley (SV) (Arora et al., 2001a). Third, the catalysts for change in India were different from those in the Silicon Valley example. The state played an indifferent role in India and did not encourage private entrepreneurial activity in the IT sector (Dossani, 2005). Fourth, domestic firms in India did not have enough access to advanced resources (for example, technical, managerial and financial), which affected their service credibility in the overseas markets (Arora et al., 2001a; Athreye, 2005). Fifth, the collaboration between industry and academia was virtually non-existent in the early stages of its evolution. Finally, there was generally an unsupportive entrepreneurial climate for private investment, and entrepreneurs had to find a "way around" the government's dominant logic of development of state-owned enterprises in the high-technology sectors (Dossani, 2005). Examining in detail now how the sector evolved will be useful when considering the review of the theoretical literature in Chapter 3.

\section{The Evolution of India's IT Sector}

Existing literature explains that the evolution and growth of India's IT sector can be broadly grouped into three main strands. Each strand of literature is critically reviewed and limitations from an organisational level analysis are presented. Its diverse theoretical orientations are grounded in literature from:

- human capital agglomeration (economics), comparative advantage, and the role of transnational corporations (TNCs) (Giarrantana et al., 2003; Patalibanda \& Peterson, 2002; Athreye, 2004, 2005)

- public policy, institutional dynamics, and evolving service capability (Athreye,2005; Arora et al., 2001, 2006; Dossani, 2005; Nilakant, 2005)

- strategy, resources, organisational capabilities, and dynamic coordination (Athreye, 2005; Banerjee, 2004; Ethiraj et al., 2005).

\section{Human Capital Accumulation, Comparative Advantage, and the Role of TNCs}

Popular press and academics have argued that the presence of certain factor endowments such as low cost, English-speaking technical human resources (Heeks, 1996) and 
geographical and time advantages favoured India's IT sector (Arora et al., 2001; Arora \& Athreye, 2001; NASSCOM, 2000). Patalibanda \& Peterson (2002) and Giarrantana et al. (2003) argue that initial factor endowments and related arbitrage opportunities led to the entry of TNCs. Their entry had a cumulative impact on the overall stock human capital because of knowledge spillovers to domestic firms from TNCs. Giarrantana et al. (2003) advance three ways that such knowledge from multinational companies spills over to domestic firms:

1. by demonstration effects and imitation through working closely with clients and learning their standard operating procedures (Gorg \& Strobl, 2002a)

2. by labour turnover from multinational companies to domestic firms when experienced employees leave (Banerjee, 2004; Gorg \& Strobl, 2002b)

3. by increasing competition and forcing the domestic firms to increase their efficiency (Caves, 1974).

These views have their detractors (Athreye, 2004, 2005; Subramaniam, 1992; Nilakant, 2005). There was no competition for the software sector, as there was no planned effort by the government to invest in human capital (Dossani, 2005). Instead, it was the hardware sector that caught the government's attention and resources in a planned manner (Subramanian, 1992). Further, if human capital agglomeration was the case, why did firms in other sectors, such as pharmaceutical sector, not mirror the performance of the IT sector (Athreye, 2004; 2005)? This question is partly answered by the changing role of the state, institutional dynamics, and the evolving organisational capabilities of domestic IT firms.

\section{Public Policy, Institutional Dynamics, and Evolving Service Capability}

Although Dossani (2005) generally argues that the state has played an indifferent, and sometimes even hostile, role in the promotion and support of the IT sector, Nilakant (2005) argues that the state has played an active role, whereby certain key actors and institutional dynamics led to the development of the sector. Using the institutional dynamics framework developed by Scott et al. (2000) and Davis et al. (2005), Nilakant (2005) argues that the growth of the sector can be broadly attributed to the role certain key actors played in influencing the dominant logic of the government at that time to adapt its governance structures to deal with the industry's development and growth needs. The 
dominant logic and governance structures prior to liberalisation programmes were: centralised planning, import substitution, and self-reliance. There were certain actors who convinced the government to change its dominant logic and governance structures at various stages of the evolution and growth of the sector.

Nilakant's (2005) phases of growth correspond very closely to Athreye's (2005) four phases of evolving capabilities of domestic firms of India's IT sector. Instead of two separate accounts, an integrated and coherent account of the sector's evolution and growth is presented. This approach is important for this research as the changing emphases noted here reflect the temporal dimension of certain organisational and human capabilities and skills developed at different stages of the sector's evolution. Thus, they reinforce the need to consider a temporal dimension and the contextual factors that drive investment in training and development at an enterprise level.

While Athreye (2004) acknowledges the initial role of agglomeration economies in human capital in the entry of TNCs and the consequent benefits that the sector derived from TNCs' entry, they weren't sufficient to sustain the increasing competitive pressures from tight labour markets due to foreign competition. Athreye $(2004,2005)$ argues that the growth was an outcome of enhanced and unique organisational capabilities developed by domestic Indian firms through their continuous improvisation on the existing business model of TNCs. With the increasing competition in factor markets for both domestic firms and TNCs and the declining billing rates for offshore software services (Singh, 2003), how did domestic firms continue to demonstrate increases in productivity (see Table 3)?

Athreye (2005) argues that because firms in the IT sector were competing with TNCs in the factor markets only and had no competition from them in the product markets, locally and globally, at least in the early stages of their evolution, domestic firms grew and increased their productivity by improvising their organisational capabilities and refining on the business model originally developed by the TNCs. It was this ability of domestic firms to transform and recombine engineers' basic skills and knowledge acquired from the factor markets into better organisational capabilities that allowed such sustained growth. 
According to Athreye (2005), such organisational capabilities include tighter project management and the development of standardised and proprietary tools for efficient deployment of resources. This capability evolved over a period of time and went through four distinct phases of evolution, each phase forcing domestic organisations to improve upon the basic business model, not only to differentiate them from competition but also to increase their productivity. It is further argued that given the generic nature of some of these capabilities, external spillovers allowed for the development of small firms in the sector.

\section{Table 3}

India's Rising Productivity for Software Revenue per Employee

\begin{tabular}{|lcr|}
\hline Year & Employees & Avg. Rev/Employee (\$) \\
\hline $1993-94$ & 90000 & 6198 \\
\hline $1994-95$ & 118000 & 6998 \\
\hline $1995-96$ & 140000 & 8924 \\
\hline $1996-97$ & 160000 & 11036 \\
\hline $1997-98$ & 180000 & 15000 \\
\hline $1998-99$ & 250000 & 15600 \\
\hline 2000 & 284000 & 32,635 \\
2004 & N.A. & 35,362 \\
\hline
\end{tabular}

(Arora et al., 2000 \& Dossani, 2005)

(Avg.re/employee data for 2000 and 2004 is from Dossani, 2005)

Nilakant's (2005) institutional dynamics model and Athreye's (2005) stages of evolving organisational capabilities and development are presented as follows.

Pre-1984

This was the period when the government's dominant logic was import-substitution and self-reliance (Nilakant, 2005). It was also a period of excessive regulation, and it focused exclusively on the development of the hardware sector and supporting state-owned enterprises for IT services (Dossani, 2005). During this period, IBM had to exit from the Indian market owing to the government's regulatory policies. In 1978, IBM quit because the Government's Foreign Exchange Regulation Act (FERA) required IBM to dilute its equity (Nilakant, 2005; Athreye, 2005). With IBM's departure, there was a void in the market for servicing the mainframes and legacy systems it had installed in the domestic 
market. Twelve hundred ex-IBM engineers were employed by a newly formed stateowned enterprise, Computer Maintenance Corporation (CMC), to service the void in the market. Domestic Indian firms were barred from operating in this area. As a result, CMC dominated this niche. However, in the medium term, some knowledge spillovers helped domestic firms to develop capability for local and overseas markets in the area of IBM mainframes installed globally. This capability proved to be extremely beneficial for dealing with legacy systems and migratory projects in the second phase of growth (Nilakant, 2005). This was also the period when there was a move from mainframes to workstations and the development of software could be undertaken independently of hardware platforms (Dossani, 2005). The above period fostered red-tapism and corruption in the government's channels. Domestic firms faced cumbersome regulations and procedures for small hardware and related equipment imports. "Red-tapism" reduced domestic firms' ability to compete and further damaged their already poor credibility. During this phase, software exports were negligible, although some firms were beginning to secure business from overseas.

\section{$1985-1991$}

This was the phase of the worldwide crash of hardware prices and a move away from mainframes to client server systems. The period saw an increased demand for customised software applications development, which coincided with the Indian Government's 1991 reforms and liberalisation programme (Athreye, 2005). Year 1991 was also the time when a change was needed in the dominant institutional logic to enable further development of the IT sector, especially to reduce the high tariffs for importing hardware (Nilakant, 2005). Certain key actors in the government, including the then Prime Minister, Rajiv Gandhi, were influential in changing the dominant logic and liberalised policies to meet the sector's hardware and software needs. During this period, Tata Consultancy Services (TCS), one of India's largest domestic ITSS firms, under the leadership of F. C. Kohli, pioneered the "onsite" business model, described by some as "body-shopping". TCS won two large contracts in the United States: American Express Incorporated and Institutional Group \& Information Co. (IGIC). The nature of contracts for projects was time and materials $(\mathrm{T} \& \mathrm{M})$ projects, as TCS's reputation in the overseas markets was poor. Onsite business models involved firms sending programmers to clients' sites for projects and most learning was "learning by doing" and by working closely with clients. The onsite model led to the development of capabilities such as working closely with clients at their site and understanding their business model and their unique cultural environment. This 
model filled in the credibility gap because by working closely with clients, communication and conflict resolution were more quickly achieved. During this phase, long-term software services contracts secured by large players and their capability to deliver cost-effective and quality services increased India's credibility as a global destination for ITSS.

Also, during this period the onsite model was beginning to be challenged by external forces, mainly by US legislative changes. The US Government enforced tighter H1B visa quotas and stringent qualification norms, and imposed market rate salaries for programmers from India (Nilakant, 2005). This led to the exploration of alternative business models and a need to change the government's dominant logic. The plan of setting up tax-free software technology parks and export-processing zones with satellite communications infrastructure was mooted by a few actors in the Government of India, who were attracted to the establishment of large TNCs like Texas Instruments (TIs) and Citicorp Overseas Limited (COSL). They were allowed to use dedicated satellite links, which had been the exclusive domain of India's Department of Defence and Atomic Energy Commission. Cheaper infrastructure and satellite communications allowed faster development and, given the 12-hour time difference between India and the US, efficiency increased and turnaround times reduced. Additional satellite communications capacities from TI were allowed to be used by domestic firms, which helped them to grow further.

\section{2-1999}

This was the period of full financial liberalisation and large-scale imitative entry of TNCs. By this time, not only were onsite contracts on the decline, but the new model of Offshore Development Centres (ODCs), using dedicated satellite communication links, started gaining momentum (Athreye, 2005; Nilakant, 2005). During this period, competition intensified, especially for domestic firms who were competing with TNCs in the factor markets and who had limited finances for setting up their own satellite facilities. As a result, domestic firms had to improvise on the current ODCs and onsite models. One such strategy was to use "quality" signalling by adopting quality management frameworks such as the Capability Maturity Model (CMM) developed by Carnie-Mellon University's Software Engineering Institute (SEI). The SEI-CMM quality framework has Levels 1 to 5, and it focuses on improving process maturity for software development, as well as on developing better organisational and management capabilities. It resulted in a redefined ODC model and productivity improvements. With better 
organisational capabilities and credibility signalling through quality certifications, domestic firms began to undertake risky but more lucrative fixed-price (FP) contracts.

\section{0 to the present}

This time has been one of consolidation and temporary correction in global software demand. It has also been the period of the dotcom crash and shakeouts in the industry. During this phase, most large domestic firms have established their credibility, increased their internal capabilities in software development and process control, and started moving up the value chain of the software development lifecycle for high-end software services, such as product design, development, and embedded software services. During this period, India's telecommunications sector, including its Internet services, has been deregulated (Athreye, 2005). Private investment in telecommunications has led to an increased telecom infrastructure and bandwidth. As a result, there has been a huge expansion in the ITeS/BPO services, such as call centres and business process outsourcing centres. The number of new players has increased significantly, and even existing ITSS players have added ITeS/BPO service lines for servicing existing and new clients. The offshore business model has allowed huge productivity gains as some large firms have begun to explore and demonstrate the "Babbage Effect", wherein firms are able to split tasks into high- and low-end and pay workers according to the complexity of the task. High-end tasks are assigned to highly qualified and experienced staff and routine, low-end tasks to less qualified staff (Athreye, 2005). Grouping and streaming of such tasks requires the development of certain organisational capabilities to pack and unpack the resources. To summarise, Nilakant's (2005) institutional dynamics framework and Athreye's (2005) explanation of the evolution of capabilities provide us with a better understanding of how the sector has come of age. The need to manage rising costs and employee turnover led to the adoption of certain HRM practices and process management strategies, including:

- investing in corporate training and education

- use of proprietary tools

- knowledge diffusion

- adopting quality standards

- recombination of resources

- innovative reward systems. 
Overall, the focus of developing such organisational capabilities was on software process control and large-scale labour management practices (Athreye, 2005). Although there is general agreement on the role of evolving capabilities and the supportive role of the state in the later stages of their evolution, little is known of how the firms developed these capabilities. More specifically, what are these capabilities, where do they come from and why do they matter? Recent research (Banerjee, 2004; Ethiraj et al., 2005) has shed some light in this area.

\section{Strategy, Resources, Organisational Capabilities, and Dynamic Coordination}

The notion of capabilities is debated and understood differently in different theoretical orientations and focuses on aspects of capability development such as organisational, economic, individual, human, and dynamic capabilities (Barney, 1991; Bryson \& O’Neil, 2008; Eisenhardt \& Martin, 2000; Teece et al., 1997; Winter, 2003). Some have argued that capability is the ability to deploy resources (Amit \& Shoemaker, 1993). Ethiraj et al. (2005) argue that capabilities reflect assets that can generate rent for the organisation. They point to capabilities as quasi-rents as they are an outcome of assets embedded in the organisational context and that the optimal use of such assets is dependent on the presence of other assets such as, managerial capital, appropriate culture and technology, or an ability to effectively put to use a bundle of these organisational assets (Ethiraj et al., 2005).

Having analysed project level data of a large Indian ITSS organisation, Ethiraj et al. (2005) concluded that firms develop certain capabilities best through "learning by doing" and that capabilities evolve over a long period of time. Such capabilities are unique and heterogeneous in nature and cannot be acquired by other firms from factor markets. Thus, they generate rent for the organisation. Further, each organisation has the choice of developing certain capabilities, as different capabilities generate different rents. The decision to invest in a particular capability is partly informed by the demand for, and supply of, software services in the market. The demand for software service, as we know, exists on a continuum, from low-end to high-end, and the corresponding service capability requirements have also changed (Athreye, 2005). On the supply side, the nature of the software contract (T\&M and FP) and the delivery model need consideration. Ethiraj et al. (2005) argue that onsite delivery generally led to the development of clientspecific capabilities, which occur as a result of repeated interactions with the client and of 
understanding better their unique requirements, and of then making changes to ensure that these requirements are met. In the case of the offshore delivery model, tight project management capabilities are needed to ensure the delivery is on schedule in terms of time, cost, and client specifications. On the second issue of whether the nature of the contract was T\&M or FP, Ethiraj et al. (2005) argue that the former led to the development of tighter project management and strong client-specific capabilities, whereas the latter led to the development of strong project management and generic people management capabilities.

In a similar vein, Banerjee (2004) argues that while resources, skills and knowledge are important, how a firm combines and recombines its resources and competencies to generate higher level competencies for achieving competitive advantage is crucial. Banerjee (2004) classifies competencies into three groups: first-order, second-order and core competency. First-order competencies are available from factor markets and every firm can easily acquire them. Second-order competencies are those that are generated by recombining the first-order competency with other resources and rules of the organisation. Using data from small domestic IT start-up firms, Banerjee argues that the core competency of an IT firm is its higher level competency, which is a decision capability because it allows switching from a project to product environment and viceversa. In explaining the growth of India's IT industry, Banerjee $(2004,2006)$ advances two key points: a job-switching thesis and the importance of dynamic coordination for information-in-expectation to yield a novelty or surprise profit. The job-switching thesis argues that human resources with medium to high experience in a given domain can provide novel information that is of value to the hiring firm and may provide an opportunity for growth. These human resources are carriers, as well as generators, of such information-in-expectation and, in Schumpeterian terms, bring surprise profit to the firm. Banerjee (2004) further argues that domestic software firms in India are opportunityseeking and look for dynamic coordination between client firms and suppliers. Banerjee (2004) notes such opportunistic behaviour from the financial investments, wherein the financial reserves of software firms are invested in highly liquid money market instruments as well as having a relatively high level of buffer employees ("employees on bench time") waiting to be deployed for a new project. A software service or product firm seeks coordination amongst software-writing firms and the users of its software through strategic demand management of services by influencing the client organisations' strategic environment. Firms first align to a clients' strategic milieu, and then, by working 
closely with them, influence and change the strategic environment of the clients. Eventually, the service provider firms modify their own strategic environment to service the clients' needs, sometimes using their higher level switching competence, productservice-product competence, as noted above.

While the above accounts have provided us with an understanding of how the sector has evolved over the last three decades, there is scope for further differentiation in the period from 2000 onwards. It has been assumed to be a homogeneous period in the evolution of the sector, despite the recent changes it has witnessed in the revenue composition of ITSS (projects and products) and an emerging value chain in the ITeS/BPO sub-sector (Dossani, 2005; Arora, 2006). An acknowledgement needs to be made given the diversity of business models and increasing capabilities to undertake high-end work (2003 onwards). Further, competition in this latter period is not only in factor markets, but also very real and intense in product markets. In this latter period also, the economy has been posting strong GDP growth rates of $8-9 \%$ per annum, which puts pressure on the overall infrastructure and resources. Although India currently spends about 3.8\% (US $\$ 8.3$ billion) of its GDP on educational infrastructure (http://education.nic.in), recent NASSCOMMcKinsey projections that by 2012 the sector will be poised to post a sales turnover of US\$85 billion and generate direct and indirect employment for about 4.5 million people seem unrealistic (NASSCOM, 2006a; MIT, 2002).

Despite the quantitative expansion of institutions since 1947, there needs to be significant investment and collaboration among all the key players in the system (for example government, employers and individuals) to sustain the projected growth rates. In this race for numbers, it is on the quality of human resources that the greatest attention needs to be focused (MHRD, 2007; NASSCOM-KPMG, 2004; NTITSD, 2003). The problem of sustainability has been further compounded in the last two years by the appreciation of the Indian rupee by about $15 \%$ against the US dollar. Medium- to long-term foreign exchange hedging contracts would provide some respite but are not sufficient. A stronger rupee will have an impact on the profitability of domestic firms and TNCs, although the impact is likely to be greater on domestic ITSS firms than TNCs, and greater still on the ITeS/BPO firms, which cater to the lower end of the market.

There have been some attempts to better understand the development of organisational capabilities, but these have been either for extremely large and high growth domestic 
firms (Ethiraj et al., 2005) or for small ITSS start-ups (Banerjee, 2004). While there is a general agreement that training and development plays an important role in the growth of certain organisational capabilities, further research is needed on the internal and external factors that drive a firm's decision to invest in training, by looking at an organisation's competitive strategy and its work organisation (Aston \& Sung, 2006; Boxall, 2003). This thesis especially examines the influence of external factors in firms operating in a dynamic outsourcing and offshoring environment, using a case-study theory-building approach (Eisenhardt, 1989).

\section{India's IT Sector: Key Conclusions}

Despite the limitations in the existing literature, some key conclusions can be drawn about the evolution of India's IT sector. They are summarised below and will, to a large extent, inform the review of the literature.

\section{Diversity}

Firms in India's IT sector have an uneven profile in terms of performance, the nature of their business models (captive, third-party, joint ventures), services that they provide (software services projects, product development and R\&D, and ITeS/BPO), their industry groups and domains and specialisations, and their mode of delivery (onsite, offshore and blended) (Heeks, 1998; Dossani, 2005, Athreye, 2005; Ethiraj et al., 2005). This distribution is important because it spells out a firm's competitive strategy (Boxall, 2003), identifies the environment (product or project) in which it wants to operate, and is likely to have an impact on the nature and extent of its training demands.

\section{Employee turnover}

Most firms in the sector report high levels of employee turnover. Employees with extensive experience and skills in a domain are seen as a source of profit by less established firms (Banerjee, 2004; Gorg \& Strobl, 2002b). High employee turnover at a lower level is an important people management issue. At both levels there are training implications.

\section{Dynamic coordination}

There is a high degree of coordination, cooperation and interdependence amongst and within firms working on various projects. This is important from a learning perspective as 
a lot of the learning that occurs in these firms is through a skills web and utilises "learning by doing" and "learning from experience" approaches. The participants in this learningsharing process include the firms' clients and suppliers (Banerjee, 2004; Ethiraj et al., 2005).

\section{Organisational capabilities}

With the evolving capabilities of domestic firms, there appears to be a temporal dimension to skills development and training (Athreye, 2005). Although Ethiraj et al. (2005) and Banerjee (2004) have identified certain key capabilities (for example, clientspecific and project management capabilities) and competencies that firms in the IT sector develop, further exploration is needed as to how these capabilities are developed and whether any new organisational capabilities are being developed now.

\section{HRM practices and linkages with training}

Given the rising productivity of firms' human resources, firms are becoming able to implement large-scale people management practices. Thus, a closer look at organisations' HRM practices, their linkages to the overall strategy of the organisation, and their training provision is needed.

Make or buy strategies (a sub-set of the above point). With intensifying competition in product and factor markets, organisations have to confront the make-or-buy decision dilemma from a training perspective. Therefore, it is important to analyse an organisation's HR strategy in terms of the level of skills they are recruiting: entry level (graduate) or lateral (experienced) hiring.

\section{Conclusion}

This chapter provided an overview of India's socio-economic and demographic features, then an account of how the IT sector of India evolved. In the next chapter, a review of literature is provided that takes into account the unique cultural and contextual factors discussed in Chapter 2 and situates the review of literature within the wider field of HRD and HRM. It also considers novel theories that might provide relevant explanations about drivers of training. 


\section{CHAPTER 3}

\section{LITERATURE REVIEW}

\section{Introduction}

The previous chapter provided a review of literature about the evolution of India's IT sector. The emergent themes from the review have implications for skills development, for example:

- the uneven profile of India's IT sector (Heeks, 1998), which suggests that differing levels of capabilities for skills development exist in the sector

- the different types of capabilities firms are developing to ensure sustained high performance (Ethiraj et al., 2005)

- the influence of human capital agglomeration (Patalibanda \& Peterson, 2002)

- investment in quality accreditation (Arora et al., 2000; Kumar, 2001; Arora \& Asundi, 2000)

- the development of a client-specific focus and strong project management capabilities

- the broad divisions in a firm's business strategy (namely a product development strategy or software services projects strategy) (Banerjee, 2004).

Given the extreme diversity in the sector and the multiplicity of growth explanations advanced, this chapter will explore in greater detail, keeping the above themes in mind, the key theoretical underpinnings of what drives a firm's decision to invest in training, with insights about how and why firms invest in training. This chapter begins with an overview of the multidisciplinary foundations of HRD, and then considers Swanson's (2007) approach for developing a theoretical framework in applied disciplines. Next, a review of theoretical perspectives that explain a firm's decision to invest in training is offered. A brief description of training dimensions and a review of specific training demand models follow. Building on useful and contributing theories and other novel theories (Swanson, 2007), this chapter provides an understanding of some specific factors identified in the literature that are significantly correlated to provision of training. The chapter concludes with the unresolved issues in the literature, especially in the context of India's IT sector, which form the basis of this study's research questions. 


\section{Theoretical Diversity in HRD}

As noted in Chapter 1, there is some agreement that HRD focuses on training and development, and career and organisation development issues for performance improvement (McMaglan, 1989; Swanson \& Holton, 2001). Swanson (2001, 2007) suggests there are three sets of underlying theories, namely, economics, psychology, and systems that help us understand the study and practice of HRD. Before reviewing the key factors and those theories identified as contributing, useful, and novel (Swanson, 2007), it is important to acknowledge the theoretical diversity in HRD research, as such. Theoretical diversity is also reflected in the extant enterprise training demand models.

\section{Unity in Diversity?}

The field of HRD is witnessing a period of intense debate. Lee (2001) refuses to define HRD on philosophical, theoretical and practical grounds. Lee considers that researchers should avoid attempts to theorise HRD because it is "necessary to avoid misrepresenting HRD as a thing of being rather than a thing of becoming", and that such attempts "also runs the risk of disengaging from the moral dimensions of HRD” (p. 331). Mankin (2001) suggests that HRD practitioners, academics and researchers should embrace HRD as an ambiguous concept. In his model, Mankin describes the relationship that HRD has with HRM and the strategy, culture and structure of the organisation and the way that they overlap with each other. Mankin further suggests that "it is this ambiguity that provides HRD with its own distinctiveness" (Mankin, 2001, p. 80).

Owing to the apparent lack of boundaries and the diversity of its conceptual bases, for example strategic HRD, knowledge management and learning organisation, attempts by practitioners, academics and researchers have been unsuccessful in establishing a conceptual framework (Garavan et al., 2000; Hatcher, 2000). Some writers from the UK consider the academic status of HRD to be problematic because of the multi-disciplinary nature of research already undertaken in this area (McGoldrick et al., 2001). Its ambiguous and problematic nature is also expressed by the use of terms such as the "emergent nature" of HRD (Walton, 1999) in defining its roots. Further, the use of metaphors for describing HRD, ranging from the River Mississippi (Willis, 1997), through three-legged stool (Swanson, 1999), to centipede and octopus (McLean, 1998), 
confirms the multi-disciplinary and evolving nature of the field of HRD. In a response to Swanson's (1999) three-legged stool metaphor, McLean (1999) says to "Get out the drill, glue and more legs", thus suggesting that the journey towards theory-building in HRD should continue and we should not limit ourselves to a few academic disciplines.

However, Swanson et al. (2000) argue that it is essential to engage in the philosophy of HRD in order to make sense of differing perspectives put forward, namely ontology (how we see the world), epistemology (how we think about the world) and axiology (the values that determine how we should act and actually act in practice and research). To this end, Swanson (2001) put forward an underlying theory of HRD, and challenged those who do not see it as essential for the development of this profession. In his work he argues, "[that] HRD should continue to mature as a discipline and that the integration of selected psychological, economic and systems theories serve as the unique theoretical foundations of HRD” (p. 229).

Given the above discourses and the ongoing theory-building efforts in HRD (Lynham, 2002; Dooley, 2002; Swanson, 1997a), Swanson (2007) makes a strong case for developing a theoretical framework for applied fields of study, such as HRD, and allowing for rival theories and other perspectives to enrich our current understanding. As Swanson (2007) puts it:

\footnotetext{
Having rival theories in a discipline is not a disturbing state of affairs. Not having a welldeveloped theory is disturbing. This holds true when framing an entire discipline or when considering even the smallest phenomenon within a discipline. The assumption is that theoretical challenges from within can only help to advance the theory. (p. 321)
}

The need for development of theoretical foundations is prevalent in applied disciplines such as management (Weick, 1979), HRD (Swanson, 1997, 2001), marketing (Hunt, 1994) and information management (Benbasat \& Zmud, 1999). However, as Swanson (2007) argues, the problem lies in integrating and interpreting the "theory discussion" and "theory research" discourse. To overcome this problem, Swanson presents a "theory framework for applied disciplines" for furthering the state of theory development and for approaching research in a holistic manner. Swanson's (2007) framework is explored later, after HRD's theoretical underpinnings have been considered. 


\section{Theoretical Foundations of HRD}

Swanson (2001) borrowed three sets of theories - one each from economics, psychology, and systems disciplines - to develop a theoretical base for HRD. In particular, the following theories were advanced as the contributing theories for HRD in Swanson's (2001) framework:

- Economics: scarce resource theory, sustainable resource theory, and human capital theory

- Psychology: gestalt psychology theory, behavioural psychology theory, and cognitive psychology

- Systems: general systems theory, chaos theory, and futures theory.

Using a general systems approach, Swanson further presented HRD processes as a performance improvement system (see Figure 5).

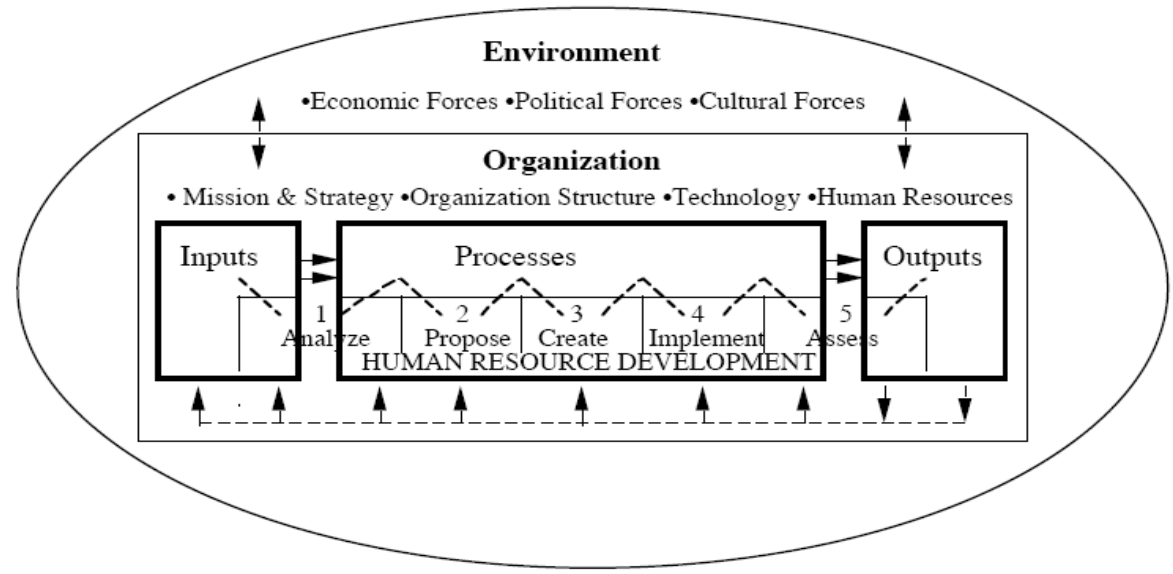

Figure 5. A systems approach to performance improvement through HRD.

(Swanson, 1996, p. 20)

In a systems model of performance improvement, which is a typical input-process-output model, the organisation is depicted as a complex, open system, wherein its mission, strategy, structure, and technology continuously interact with various sub-systems within and outside the economic, political, and cultural milieus in which it operates. Within these complex and multi-level systems, HRD is seen as a process for improving the performance of an organisation's systems and of the people working in it.

Despite the criticisms of the performance paradigm of HRD by supporters of the learning paradigm of HRD (Barrie \& Pace, 1998, 1999; Bierema, 1997; Dirkx, 1997), Holton 
(2002) offers some clarifications to remove the misunderstandings as well as to refute some of the criticisms. Holton (2002) attempts to resolve the ongoing tension between learning and performance paradigms by suggesting that the two are not mutually exclusive; rather, one leads to the other. Further, if HRD were to gain prominence in organisations, the performance paradigm must prevail for HRD to be seen as a strategic partner, one that creates added value in an organisation's performance system. Yang (2004) offers clarification and some explanation of how the two paradigms are linked and how a strategic approach to HRD is essential for a performance paradigm.

Yang (2004) argues that, as applied fields of study, HRD and adult education have to interact with adults in the organisational context for managing learning, performance and change. Further, the three concepts of learning, performance and change are related and learning is the common thread that runs through them. Therefore, it is not possible to have improvements in performance or to successfully implement change without having an element of learning experience in each. Further, as Swanson et al. (1998) conclude, despite being distinct fields of study and practice, adult education and HRD are both dedicated to adult learning; one is not a sub-set of the other. The common ground shared by these two disciplines rests at the following point:

\footnotetext{
When adult learning outcomes and learning process decisions about individuals are bounded by rules and requirements of the organisation, adult learning is HRD. When adult learning outcomes and learning process rules and requirements are located in the individual, it is adult education. (Knowles et al., 1998, p. 121)
}

This section has reviewed the key theoretical foundations, multidisciplinary nature of HRD and, has provided a short overview of the theoretical diversity that exists in the field of HRD. The following section provides an overview of Swanson's (2007) theory framework for applied disciplines.

\section{Developing Theory Frameworks for Applied Disciplines}

In the integration of the diverse theoretical viewpoints into a framework for analysis, Swanson's (2007) guidelines for developing theoretical frameworks in applied disciplines such as HRD are considered a useful starting point because they allow for any novel and emergent themes, factors, and theoretical perspectives to be incorporated in this research. Swanson (2007) proposes six components necessary for developing theoretical frameworks: boundary of the theory, contributing theories, the core theory, useful 
theories, novel theories, and irrelevant theories for an applied discipline. Swanson (2007) describes his above framework as follows:

- The boundary of the theory specifies the purpose, definition and assumptions that frame the theory.

- Its contributing theories are those that address the boundaries of the applied discipline.

- The core theory is best described as the intersection of all the contributing theories and one that addresses its purpose, beliefs and assumptions.

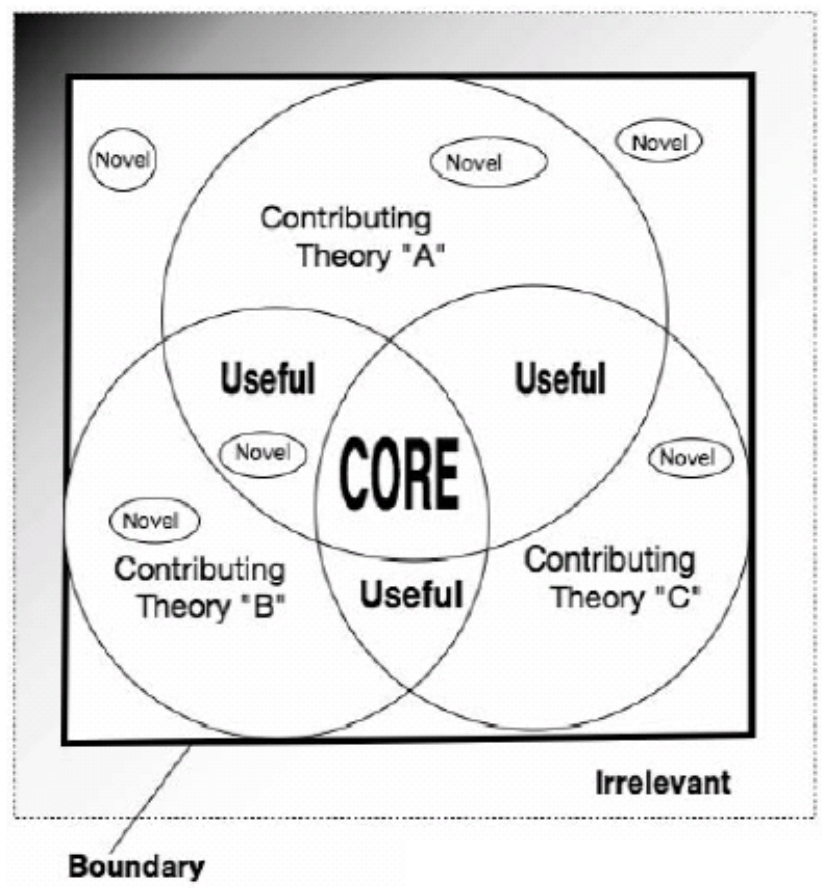

Figure 6. Theory framework for applied disciplines: Boundaries, contributing, core, useful, novel, and irrelevant components.

(Swanson, 2007, p. 328)

- Useful theories are outside the core but within the intersection of the contributing theories.

- Novel theories are theories of some narrow phenomenon that provide a unique understanding of applied discipline.

- The irrelevant components are any theories that are outside the above theories and have no potential to contribute to the applied discipline. 
Applying the above framework, Swanson (2007) demonstrates how his earlier work on the underlying theories of HRD (Swanson, 2001) satisfied the criteria listed in this framework. Given the multiplicity of theoretical bases, ongoing theory-building efforts, and Swanson's (2007) theory framework development criteria, it is desirable to include some useful and novel theories for studying the phenomenon of what drives training decision-making in India's IT sector. Including these theories is logical as the study tries to bring together theories in a discipline to meet practice and theory development demands, especially from an outward-looking perspective. See Figure 6 for a graphical illustration.

For the purposes of this study, economics, systems, psychology, management, and marketing theories are considered to be contributing theories. For the purposes of this study, and in line with the philosophical assumptions of HRD, organisations are purposive economic entities operating for a surplus. Following Swanson's boundary assumptions in framing HRD (2007) and the definition of training outlined in Chapter 2, the boundaries of this theory-building attempt focuses on developing an organisational level understanding of the internal and external factors that influence training decisionmaking. Theories based on sociological or adult education approaches or those focusing on the individual level are not included as they are not the focus of this study. The following useful theories are considered in the context of this study: the human capital theory, neo-human capital theories, HRM, high performance work practices (HPWPs), and organisational learning theories.

Given the emergent themes of investing in total quality management programmes and the development of project management capabilities in India's IT sector, the framework also includes the role of quality management for any novel explanations in the provision of training. Further, to understand how a firm develops client-specific capabilities, it is important to borrow concepts from the marketing discipline. To this end, the theory of market orientation (Kohli et al., 1993; Narver \& Slater, 1990) and learning orientation (Argyris \& Schon, 1978; Senge, 1990), which are key elements of the market-based organisational learning framework (Sinkula et al., 1997), are also considered for any novel explanations. The above approach is by no means a closed approach to guide the data collection and analysis; rather, it is a starting point and an attempt to synthesise different theoretical orientations into a coherent assemblage for understanding the phenomenon of training provision and to answer the study's questions. 


\section{Is this a Quest for a Grand Theory of Enterprise Training?}

The answer to the above question is "No". There are several reasons. First, in complex and dynamic business environments, it is not possible to capture all the nuances and facts whose "axiomatic generalisations" lead to a theory (Banerjee, 2004). Second, the predictive power of applied theories in explaining all the economic and organisational issues of today's dynamic and complex organisational systems is limited. Third, most stand-alone theories operate in a narrow space, thereby making theory development and inclusiveness almost impossible. The approach adopted here is similar to what Banerjee (2004) describes as "piecemeal engineering", as it is not based on a grand theory or two that have strong predictive powers in diverse organisational settings.

The approach adopted here is a pragmatic one, wherein, from the assemblage of certain contributing, useful, and novel theories (Swanson, 2007), explanations are sought from a participant's perspective to see how plausible, correct, and fitting they are to the theories included in the study. Any novel explanations are allowed that enhance contextual relevance. Finally, given the openness to new theoretical findings and the methodological flexibility included in the research design, it appears to be a logical way to seek an understanding of the explanations from the participants in their unique contextual settings. Before the different theoretical perspectives on a firm's decision to invest in training are explicated, an overview of the commonly noted dimensions of the nature and extent of training activity in organisations is provided.

\section{Nature and Extent of Training}

For the purposes of this study, following extant training demand models (Dawe, 2003; Dostie \& Montmarquette, 2007; Smith \& Hayton, 1999; Ridoutt et al., 2002), the extent (volume) of training is described as considering the proportion of employees trained and the percentage of payroll spent on training in a given year. Hayton et al. (1996) and Ridoutt et al. (2002), for example, found enterprise size, workplace change, workforce professionalisation, introduction of new products, and the presence of a strategic approach to training as key factors influencing training volume. The nature (diversity) of training is analysed using five dimensions of training practices: 
(1) the extent to which an organisation utilises external or internal provision

(2) formalised approach to its training needs analysis, design, development and delivery

(3) transferability of training to other firms

(4) learning support for pursing higher education qualifications

(5) type (general skills or technical skills) of training provided.

Workplace change, enterprise size, and introduction of new products was found to influence the nature of training provision in Australian and UK firms (Green et al., 2003; Hayton et al., 1996; Smith \& Hayton, 1999; Ridoutt et al., 2002). The following section provides a critical review of the main contributing, useful, and novel theories that have been identified in the literature on explaining the phenomenon of why firms invest in training and what drives their decision making. The following does not offer a comprehensive review of the contributing disciplines but instead it focuses on the review of specific theories from contributing disciplines that help further our understanding of an organisation's decision to invest in training.

\section{Firms’ Decisions to Invest In Training: Contributing, Useful, and Novel Theoretical Perspectives}

This section considers the useful and novel theories and offers a critical review of respective contributions. Where possible, this section also offers insights into the relevance of some of these theories to the Indian cultural context, especially its IT sector. First, the predictions of human capital and neo-human capital theories are examined for their usefulness to the study. The rationale for its inclusion stems from an economics and performance paradigm of HRD. Further, the mediating role technology plays in productivity calls for neo-human capital explorations. Next, owing to the complexity of organisational decision-making, the impact of competitive and business strategies, including an organisation's HRM strategies, is explored in shaping the nature and extent of training.

The presence of quality management approaches in most studies of high performance work practices (HPWPs) and the widely acknowledged role they play in achieving sustained competitive advantage is analysed. The role of quality management systems in an organisation's production function and its impact on training is also analysed (Morgan 
\& Piercy, 1996; Reed et al., 2000; Ridoutt et al., 2002; Smith et al., 2004). Next, a critique of the above theoretical perspectives is offered. Finally, considering the limitations in the above theoretical perspectives, gaps in HRD literature (Leimbach \& Baldwin, 1997; Short, 2006), and keeping in mind the dynamic nature of coordination that exists between clients and service provider firms (Banerjee, 2004, 2006), this thesis considers Sinkula et al.'s (1997) market-based organisational learning framework for any novel explanations. Sinkula et al.'s framework is useful because it is based on the assumption that certain organisational values drive organisations' behaviours and actions. Inclusion of such an approach allows for understanding the context and the learning culture in which an organisation promotes the development of certain knowledge questioning values and knowledge producing behaviours and their relationships with other organisational capabilities (Morgan \& Piercy, 1996; Day, 1994).

\section{Human Capital Theory}

Economists have stressed the basics of human capital theory (HCT) (Becker, 1964; Mincer, 1974; Strober, 1990) by considering the role of education and training as a way of increasing employee productivity. Nafukho et al. (2004) presented the implications of HCT for HRD and showed how the field of HRD can benefit from further research on advancing the state of theory. HCT started gaining momentum when economists argued for the role of education in increasing a nation's productivity (Schultz, 1960). However, it was not until Nobel laureate G. S. Becker's $(1962,1964)$ work was published that HCT was formalised. It was argued then that availability of high-end skills in an economy's human capital base is essential for its competitiveness and overall well-being. Some studies focused on the role played by intangible human capital formation in a nation's economic growth (Abramovitz \& David, 1996). The basic thrust of HCT is that, in a perfectly competitive market, continuous investment in education or employee training results in increased productivity, which results in higher earnings. This has been claimed since Adam Smith and, more recently in the development literature, since the 1960s (Shultz, 1961; Becker, 1964; Mincer, 1974).

\section{Key Relationships In HCT}

There are two main relationships advanced in HCT. First, it represents the concept of production function applied to education and training, wherein education and training are inputs for better learning and improved organisational outcomes, such as productivity. 
The main assumption in HCT is that improved learning does in fact increase productivity and that increased productivity is linked to better wages and business earnings.

The second key relationship is about investment in two types of training for individual employees: firm-specific skills and general skills. Becker (1964) was the first to differentiate between firm-specific and generic training. He suggested that firms are more likely to invest in firm-specific human capital than in generic human capital, as the former is concerned with the individual's immediate job requirements and in most cases not transferable to other organisations, whereas the latter is transferable to other employers because it is of the same value to other employers. Further, firm-specific training can be recouped during an employee's productive time, and an organisation can gradually increase the wages of its employees as it starts to recoup its investments in firm-specific training. With generic training, there is the risk of employee turnover and the benefits of the training being transferred to another employer. At a firm or organisational level, Mincer (1997) argues that on-the-job training is vital for productivity, because it raises the specific skills required in a job, which are not usually obtained in the course of formal educational or vocational courses, and that skills training is one of the most critical factors in the underlying wage structure.

\section{Empirical Evidence: Return on Investment for Employer-Sponsored Training and Types of Training}

Numerous econometric studies have been undertaken to prove the basic tenets of HCT. A resounding number of studies provide empirical evidence that investment in employersponsored training results in higher productivity and increased wages (Bishop, 1991; Bartel, 2000; Dearden et al., 2006; Lazear \& More, 1984; Maglen, 1990). In the US, for example, Bishop (1991) used data on 2594 employers to estimate the impact of the first 3 months of training on the 2-year growth in a worker's productivity. Results from the analysis indicate a marginal rate of return. For every 100 hours of training, the increase in productivity ranged from $11 \%$ to $38 \%$ for the logarithmic specification. Similarly, also from the US, Bartel (2000) studied the impact of formal training programmes in 495 organisations and found increases in productivity of about $18 \%$ over a 3 -year period.

In the UK, using the 1983-1996 panel data of British industries, Dearden et al. (2006) used direct measures of productivity to examine the effects of work-related training. They found a $1 \%$ increase in training to be associated with an increase in added value per hour 
of about $0.6 \%$ and an increase of about $0.3 \%$ in the hourly wages. Dearden et al. further argue that their contribution is unique, as it is the only study that tests the relationship using direct productivity over a 14-year period. The implication here is that using wages as the only measure of productivity runs the risk of ignoring other productivity benefits that a firm may derive. They found the coefficient of training in the production function to be twice as large as the coefficient in the wage equation. They further argue that this is a result of imperfect competition in the labour markets, a viewpoint that has been espoused by neo-human capital theorists (Acemoglu \& Pishcke, 1998a, b).

Overall, the focus of the above studies has been mainly on establishing the link between productivity, wages, and investment in training. Apart from training and education, the evidence presented may be influenced by other factors internal and external to the firm. There is also a related problem with the measures used in testing the relationships, since most studies have employed large sets of panel data, collected initially for a different purpose, with different definitions. Alternative explanations in the extant literature are the screening and signalling hypotheses (Arrow, 1973; Spence, 1973), wherein an organisation uses the incumbent employee's level of educational qualification and training as a means of filtering better educated workers into more highly paid jobs.

The second issue of investment in generic versus firm-specific training has been intensely debated in the literature on HCT. The problem evident in this approach to distinguishing training is that it is difficult to measure the gains in productivity accruing from such investments because elements of generic and firm-specific training are likely to be present in each, and the distinction between firm-specific human capital and generic human capital is blurred (Maglen, 1990; Strober, 1990). Given the business complexity and rapid technological change, it is hard to establish when one becomes the other, although, from a theoretical viewpoint, firms would want to transfer the cost of general training to employees for fear of losing out on their investments to other firms through employee turnover. Conversely, firms investing in firm-specific training will make employees relatively less attractive to other firms, given the specific use of such training. This has been confirmed by empirical evidence by Lynch (1991), wherein employees who received firm-specific training were less likely to leave the organisations. The above research suggests that firms will invest more in firm-specific training than general skills training (Proposition 1). 
However, some researchers argue that support for firm-specific training investments over generic training is also losing traction. Studies from the USA, UK and Australia (Cappelli \& Rogovsky, 1994; Osterman, 1994; Smith et al., 2003, 2004) have confirmed that firms using HPWPs, such as lean production, total quality management, team working, learning organisation, job rotation, and business process reengineering, seem to invest a lot in both technical and firm-specific training, as well as generic and transferable training. HCT has attracted further criticism from institutional economists (McNabb \& Whitfield, 1994), who argue that employee productivity and wage increases are also influenced by factors other than training, for example, the nature of the job, external labour market conditions and the need to retain employees, so that organisations have to pay them higher than external market wage rates. Despite the overall limitations of HCT, economists have called for further exploration and explanation under imperfect competition. This area is examined under the rubric of neo-human capital theory.

\section{Neo-Human Capital Theory}

Broadly, there are two conceptual divisions in neo-human capital theory. The first deals with the mediating role technological innovation plays between education and training, and productivity. Proponents of this theory argue that highly trained and educated employees are more likely to adopt technological change than less educated and less skilled employees (Wozniak, 1984, 1987; Bartel \& Lichtenberg, 1987) and that new technology is a means of improving productivity. Further, the demand for highly educated workers would decline with increase in experience on a particular technology (Bartel \& Lichtenberg, 1987). Such thinking arose during the time that most sectors in developed economies were undergoing technological change.

The second stream of neo-human capital theory challenges the orthodox views of HCT. Becker's HCT is based in part on the premise that, in a competitive labour market, the wage rate equals the marginal product of labour. Two implications of this premise are that (a) a worker receives all returns from general training and (b) firms will not pay the cost of general training. Notwithstanding these long-held beliefs, recent empirical studies, including those conducted by Acemoglu and his colleagues at MIT (Acemoglu \& Pischke, 1998a, 1998b), have found that many firms are, in fact, willing to share in the cost of general training. Acemoglu and Pischke explain this paradox as an artefact of information asymmetries or market imperfections created by the inability of the firm to 
observe the true abilities of workers. This explanation can be viewed as an elaboration of Katz and Ziderman (1990) and Chang and Wang (1995, 1996), who argue that if outsiders are unable to observe the amount of investment in human capital, the firm may pay for general training. More generally, neo-human capital theory posits that the alternative wage a trained worker can receive from another employer is limited by the informational monopsony power of the current employer and that this power imbalance offers the firm an incentive to invest in general training. Acemoglu and Pischke further argue that where transactions costs (trading costs) exist and in labour markets affected by the creative destruction effect of (post-industrial) skill-biased technological change (which is induced by increased international trade), firms are willing to pay for general training since they can share the returns from the increased productivity accruing from the training enhancements (as has long been said, under traditional human capital theory) of firm-specific training. The above view gives rise to the second proposition: that firms will invest in general skills training due to asymmetrical market information firms have about their new employees (Proposition 2).

\section{Empirical Evidence for Neo-Human Capital Theory}

In an agricultural setting, Wozniak (1984) found support for his hypothesis that farms wanting to adopt new technical innovations have to rely on their human capital endowments. In particular, the presence of educated workers and access to the information about the technical innovation helps in reducing the costs of, and uncertainty about, adopting them. Wozniak found variance across farm size, wherein producers with larger scales of production were more likely to adopt technical innovations than their smaller counterparts. Wozniak (1987) undertook further analysis and found that the ability of firms to adopt technical innovations to overcome rapid technological changes in the external environment depends on the index of education, experience and information that they possess about the innovation, which is also a firm's overall innovation ability. He further argued that difference among firms' performance of innovation arises out of their innovative ability because their ability to decide about adoption of innovations is a function of that innovative ability, which includes human capital intensity, experience, and the ability to sense, collect, and analyse information about a particular innovation. The proxy measure for human capital intensity used by Wozniak is the level of education, whereby a highly educated agent's ability to obtain, decipher, and appreciate information relevant to a given innovation is greater than that of less educated workers. Organisations with higher levels of human capital intensity would know where to look, and how to 
access information that would help them in innovation adoption decision-making. Wozniak (1987) further hypothesised that those who have a higher level-of-education index and who are producers with higher levels of experience, training and learning, and information-sensing and analysis ability are more likely to be innovative, relative to their less experienced and less skilled counterparts.

Bartel and Lichtenberg (1987), building on the earlier work of Nelson and Phelps (1966) and Welch (1970), hypothesised that educated workers have a comparative advantage over less educated workers with respect to adjustment to, and implementation of, new technical technologies, in that their demand, relative to less educated workers, decline as the age of the plant and equipment increases. This comparative advantage is even more pronounced for sectors undertaking R\&D work. In other words, as an organisation introduces a new process or technology, the transition from the old to the new technology is slow and ill defined, at least initially. The processes are not so well specified or broken down into simpler tasks to the same extent when one gains experience using that particular technology. Others (Bright, 1961; Nelson et al., 1967) have observed that the skill requirements in several industries first increased and then declined as mechanisation increased. These findings suggest that the demand for highly skilled workers will decline with experience on a given technology (Proposition 3).

Using neo-human capital theory assumptions, OECD researchers at the Centre for Educational Research and Innovation (CERI) investigated technological change in the manufacturing (automobiles) and services (banks and insurance companies) sectors in France, Germany, Japan, Sweden, and the USA to establish the link between training and education and a firm's ability to adopt technological change. OECD/CERI research (1986, 1988) found four key factors that affected the development of a skilled and flexible workforce. They found adoption of technology, the nature of work organisation (especially where there was a move from a Taylorist paradigm to one that had high involvement and team-based structures), cooperative industrial relation systems, and the presence of skill formation systems to be vital to training effectiveness. They also found that these factors are intertwined with an organisation's strategy. The impact of technological change on training is also noted in a study done by Bartel and Sicherman (1995). Using the National Longitudinal Survey of Youth (male workers) in the US, Bartel and Sicherman (1995) found that, while it is likely that highly educated workers 
get more training, the gap between training provision for highly educated and less educated workers narrows as the rate of technological change increases.

In a study of 1941 employers in fifteen trades, the Canadian Apprenticeship Forum (2006), using a cost-benefit model, reported that the employer generally gained $\$ 1.38$ for every $\$ 1$ spent on an apprentice. Also from Canada, Turcotte and Rennison (2004), using data on 4,219 locations from the 1999 Workplace and Employee Survey, developed by Statistics Canada and Human Resource Development Canada, examined (a) the link between adoption of new technology and productivity; (b) whether productivity benefits are greater when investments in technology are combined with investments in education and training; and (c) to what extent productivity changes resulting from the adoption of technology and investment in human capital are associated with increased wages. Turcotte and Rennison (2004) found that use of technology was more prevalent in highly productive locations, although there is no statistically significant relationship between technology use and clusters of high and low productivity locations. They also found that university education and computer skills training are positively associated with increased productivity and that productivity returns to firms are much larger than wage returns to workers, especially in the case of computer-based training.

Smith and Dowling (2001), in a case study of seven Australian firms, found support for the above neo-human capital assumptions and also highlighted the importance of a firm's strategy, which is linked closely to its work organisation, technology adoption, and other people-management practices, in establishing the nature and extent of enterprise training. A firm's competitive strategy impacts on the nature of products and services in an organisation's production mix. For example, the role of strategy in following a high or low skills and quality path was considered in a study of British and German enterprises operating in casual apparel and furniture manufacturing (Steedman \& Wagner, 1987). In both industry sectors, Steedman and Wagner found the adoption of a strategy to service niche markets for high quality products by German enterprises (as compared to the low skill and mass production market strategy by British enterprises) was a key factor in explaining utilisation of skills and their development.

There are limitations in the neo-HCT school of thought because it does not consider the impact of other institutional dynamics or the nature of product market strategy. For example, researchers from the UK (Finegold \& Soskice, 1988; Finegold, 1991, 1992) 
modelled the role of institutional dynamics in education and training provision, using game theory. Finegold emphasised the need for collaborative working among policy makers, employers and individuals for effective, long-term skills development. Finegold highlighted the need for Western and English-speaking economies to move away from their short-term focus of ensuring maximum returns to their shareholders at the cost of skills development. Finegold supported his thesis by suggesting the success of a longterm skills development strategy adopted by Germany and Japan.

Although the above studies provide insights about the role that skills and education play in the adoption of technical innovations, they lack any reference to the contextual environment in which the whole production function is organised. They do not also consider, for example, the impact of product life cycles on training and education (Ottosson \& Wang, 1997). Ottosson and Wang found in the case of high technology organisations, where product life cycles are short, that the level of investment in education and training increased on an annual basis.

Another dominant strand of literature has been the role of strategy, HRM and implementation of high performance work practices (HPWPs) in developing skills. Although HRD, which encompasses training and development, has evolved as a separate field of study in itself, HRM literature considers HRD its sub-set. The following section reviews this overlap and HRD's relationship with HRM and an organisation's competitive strategy in informing its training provision.

\section{Human Resource Management, Business Strategy, and Training}

As it is for HRD, theory-building debate in HRM is still ongoing. Although some academics have presented their conceptualisations of HRM, consensus is far from being reached. While Mankin (2001) explains the overlap between HRM and HRD, others have generally emphasised the much noted training-performance paradigm, where a bundle of HRM practices are posited to be the missing link for better performance (Boxall \& Purcell, 2003). Broadly, the dominant perspectives in HRM can be classified into three main groups: best-practice HRM, best-fit HRM, and the resource-based view of the firm. Other classifications include: hard or strategic HRM models, soft HRM models, and the unitarist and pluralist views of HRM. The following section first provides a discussion of 
the three main conceptual divisions in HRM and then considers the links between HR strategy, HPWPs, and training.

Research within the best-practice tradition (Beer et al., 1984; Kochan et al., 1986; Pfeffer, 1998) is akin to studies of HPWPs (Applebaum et al., 2000), high-commitment HR (Guest, 2001), or high-involvement (Wood, 1999) in the extant literature. In this tradition, the thrust is on improving performance through a "bundle" of universally applicable HR practices. High performance in the HRM context, among other outcomes, refers to low employee turnover, high quality HR service delivery, improved skills capability, strategic integration, and improved attitudes and behaviours. Such performance is seen as instrumental in increasing the organisation's productivity. For example, Purcell et al. (2003) point out (using the classic performance equation $(\mathrm{P}=f \mathrm{AMO})$ ) that high performance can be achieved by a set of 11 interrelated human resource practices, which include learning and development and performance and development appraisals. Purcell et al. (2003) highlight the role of line managers in implementing such practices successfully. However, critics of best-practice models (Marchington \& Grugulis, 2000) challenge the universal claims espoused by these models, given the high level of complexity in the real business world and the need to accommodate an organisation's contextual factors and employee voice in developing any HR practices. Further, application of some of these models may be possible only for large and diversified businesses given the implementation costs. Finally, implementation and adoption of such practices is also dependent on the strength of an organisation's capabilities, a view that is consistent with resource-based view (for example, Barney, 1991).

Resource theorists (Barney, 1991; Leonard, 1998; Wernerfelt, 1984; Wright et al., 1994) argue that an organisation's human resource is one of the many resources it can exploit in its production function to achieve sustained competitive advantage. The resource-based view suggests the development of certain competencies that are critical for an organisation to achieve sustained competitive advantage. Barney (1991) popularised the resource-based view of the firm. Hamel and Prahalad (1990) developed the notion of core competencies and argued that core competencies are learned attributes that provide a firm with long-term competitive advantage. These competencies can be developed only over the long term. For an organisation's resources to deliver sustained competitive advantage, the resources must add value to the firm, be unique or rare, and be imperfectly inimitable, that is, unable to be substituted with other resources or provided by competing firms 
(Barney, 1991). Among other capabilities, this view requires development of certain organisational practices that support training and skills development, retention and focused recruitment, and management of the tacit and explicit knowledge that is critical for the achievement of sustained competitive advantage.

According to Banerjee (2004), resource theorists offer significant conceptual limitations, as most knowledge and physical resources can be procured from the market and an organisation's unique path dependencies can be transferred to other organisations through job switching and its market information-sensing capabilities (Sinkula et al., 1997). It can be further argued that tacit or explicit knowledge cannot be a source of competitive advantage. If it is explicit and codified, then it is portable to all and is a market good, unless protected by patents. If tacit knowledge exists and resides in the minds of the individuals, then, although difficult to imitate, it can arguably be transmitted through the individual carriers of such knowledge, by virtue of job switching, to rival firms that could potentially profit from such knowledge (Banerjee, 2004).

Best-fit models of HRM adopt a contingency-based approach and stem from the premise that each organisation operates in a different strategic milieu, which affects its work organisation and product and/or service market portfolio. Therefore, each organisation should develop its human resource strategy in line with its business strategy and look for a fit between the two. Best-fit models are generally seen as having a closer link with the dynamic external environments.

Best-fit HRM models can be classified into three groups. First, HR practices should be based on the business life cycle model (Baird \& Meshoulam, 1988), wherein an organisation goes through different phases of growth and each phase has a distinct set of HR practices that need to be developed and delivered. For instance, an organisation's training and development needs would be high during the start-up stage compared with the later, mature stages. Second, HRM practices focus on developing effective strategic and structural configurations. Fombrun et al. (1984) highlighted a strong need for an internal strategic fit and identified a range of strategic HR choices in recruitment, selection, training and development, and rewards and remuneration, depending on the firm's strategic configurations. To this end, training activities should be closely aligned with an organisation's strategic needs. Finally, best-fit models draw upon Porter's (1985) 
classification of three generic strategies: innovation, quality enhancement and costreduction.

Schuler and Jackson's (1987) work has been considered to be dominant in this group. In their work, Schuler and Jackson argued that firms could pursue any of the three strategies and that HR strategy should be geared towards identifying key behaviours from which an organisation would gain value, and would need to support each of the three strategic orientations. The competitive strategy adopted by an organisation would have varying training demands. For instance, in case of a cost-leadership strategy, training and skills development would not be high on an organisation's agenda.

Best-fit models are not free from detractors, as they are often labelled as top-down and prescriptive. Further, although a simple strategy typology is advanced for developing strong internal fits, many organisations follow a more pluralistic approach to strategy and may have elements of all three strategies in operation at the same time. Second, as Boxall and Purcell (2003) argue, organisations do not exist in a vacuum; they are rooted in societies and are also influenced by institutional forces, which ultimately define the strategic milieu in which they are operating. Finally, it is not possible to factor all the contingent variables, their interrelationships and the paths they follow to establish clearly defined HRM-performance links (Purcell, 1999). To this end, the following section provides further discussion on the links between an organisation's competitive dynamics, market characteristics, and the HR strategy it can adopt, especially in service industries.

\section{Market Characteristics, Competitive Dynamics, and HR Strategy}

Boxall (2003) provides a framework for understanding the market characteristics, the competitive dynamics and an organisation's HR strategy in the services sector. Building on Herzenberg et al's (1998) typology of differentiated work systems-ranging from highly constrained and Taylorised forms to high-skill autonomous structures - and using Porter's classification of strategies (Porter, 1985) on the nature of markets served, Boxall developed a typology of three types of market that firms can get into and identified the nature and the basis of competition and work organisation for each type.

The three markets identified are: mass-service markets, a mix of mass markets and higher value-added segments, and very significantly, if not totally, differentiated markets. Within these market segments, Boxall (2003) identified the nature of knowledge content, 
typical work design, competitive dynamics, and the predications for appropriate HR strategy (see Figure 7 for more details). Boxall's (2003) classification suggests that there are opportunities to implement HPWPs (including quality management approaches) in Type 2 and 3 markets or where there is higher value-added economic benefit available. Second, he argues that the skills that firms should possess for HPWPs' implementation need not be high as long as there is an economic justification for implementing HPWPs.

\begin{tabular}{|c|c|c|c|c|}
\hline SERVICE MARKET TYPE & $\begin{array}{l}\text { KNOWLEDGE } \\
\text { CONTENT OF SERVICE }\end{array}$ & TYPICAL WORK DESIGN & $\begin{array}{l}\text { COMPETTIVE DYNAMICS } \\
\text { IN THE SECTOR }\end{array}$ & $\begin{array}{l}\text { PREDICTIONS For HR } \\
\text { STRATEGY IN FIRMS }\end{array}$ \\
\hline $\begin{array}{l}\text { Type } 1 \\
\text { Mass-service markets } \\
\text { (eg petrol stations, fast } \\
\text { food, supermarkets) }\end{array}$ & $\begin{array}{l}\text { Low. Key managers or } \\
\text { franchisees have critical } \\
\text { knowledge, but general } \\
\text { labour uses limited, mostly } \\
\text { generic 'know-how' }\end{array}$ & $\begin{array}{l}\text { Low discretion. May be } \\
\text { highly 'Taylorised' in } \\
\text { international franchises } \\
\text { or major chains; } \\
\text { otherwise unrationalised, } \\
\text { low-skill work }\end{array}$ & $\begin{array}{l}\text { Cost-based except to the } \\
\text { extent limited by unions and } \\
\text { state regulation; substitution } \\
\text { of labour for technology and } \\
\text { self-service; some branding } \\
\text { strategies possible }\end{array}$ & $\begin{array}{l}\text { Firms typically fit HR } \\
\text { strategy to their cost-driven } \\
\text { competitive strategies } \\
\text { through paying only the } \\
\text { market-clearing wage and } \\
\text { complying minimally with } \\
\text { labour laws; very limited } \\
\text { prospects for HR advantage, } \\
\text { except where premium } \\
\text { brands can be created } \\
\text { and sustained }\end{array}$ \\
\hline $\begin{array}{l}\text { Type } 2 \\
\text { A mix of mass markets } \\
\text { and higher value-added } \\
\text { segments (eg elder care, } \\
\text { hotels, call centres) }\end{array}$ & $\begin{array}{l}\text { Low-to-moderate knowledge } \\
\text { levels mix of skill level } \\
\text { needed in the workforce }\end{array}$ & $\begin{array}{l}\text { Traditionally low-to- } \\
\text { moderate discretion, but } \\
\text { potential for job enrichment } \\
\text { and HPWSs }\end{array}$ & $\begin{array}{l}\text { Mix of cost and quality- } \\
\text { based competition; greater } \\
\text { profit opportunities for firms } \\
\text { that identify higher value- } \\
\text { added segments }\end{array}$ & $\begin{array}{l}\text { In mass markets, HR } \\
\text { strategies are Type 1, but } \\
\text { possibilities exist for HR } \\
\text { advantage in higher value- } \\
\text { added segments; potential } \\
\text { problems with imitability } \\
\text { and appropriability }\end{array}$ \\
\hline $\begin{array}{l}\text { Ty pe } 3 \\
\text { Very significantly, if not } \\
\text { totally, differentiated } \\
\text { markets (eg high-level } \\
\text { professional services) }\end{array}$ & High knowledge intensity & $\begin{array}{l}\text { High discretion - } \\
\text { the natural home of } \\
\text { HPWS }\end{array}$ & $\begin{array}{l}\text { Expertise and quality-based } \\
\text { competition, but with some } \\
\text { anchors on relative pricing } \\
\text { some services may be } \\
\text { routinised and migrate back } \\
\text { to Type } 2 \text { competition }\end{array}$ & $\begin{array}{l}\text { Extensive opportunities for } \\
\text { HR advantage in expertise- } \\
\text { driven niches; potential } \\
\text { problems with imitability } \\
\text { and appropriability; use of } \\
\text { lower cost HR strategies } \\
\text { where expertise is routinised }\end{array}$ \\
\hline
\end{tabular}

Figure 7. Market characteristics, competitive dynamics, and HR strategy in services.

(Boxall, 2003, p. 13)

Although the above is a useful classification of the market types, it is simplistic because, in the real world, a number of large and diverse organisations are likely to be operating in all of the three types of market at the same time for different products and services. What is also not clear from this classification is the nature of the specific HR practices that an organisation may adopt. For example, from a training perspective, will an organisation invest negligible amounts in training if it belongs to Type 1 market segment and more if it belongs to Type 3, and if it does, what type of knowledge and skills are developed? Also, will firms in a Type 1 market rely on recruiting skills from external labour markets, and will they develop it in-house in a Type 3 market? Is a high skills road necessary for a Type 3 market? Evidence from the UK does not seem to support the need for a high skills road for a differentiated product market (Mason, 2005). Likewise, in another study, a 
differentiated product market was not found to be associated with the use of highly skilled labour (Sung \& Ashton, 2005).

In terms of market characteristics, competitive and HR strategy for ITeS/BPO (especially call centres) and software development, Batt and Moynihan (2002) and Banerjee (2004) offer useful classifications. Batt and Moynihan advance three call centre production models: a classic Taylorist mass production model, a professional service model, and a mass customisation model. The first and second models of production correspond closely with Boxall's (2003) production models and his classification of Type 1 and 3 markets, with the mass customisation coming close to the production model typical of Type 2 markets. The implications for skills development advanced by Batt and Moynihan are not very different.

The above classification is useful for studying call centres and BPO organisations in India. However, for IT software services and product development firms, Banerjee's (2004) approach to understanding competitive strategy and market characteristics appears to be pragmatic. As noted earlier, Banerjee offers evidence for two broad divisions in the Indian IT sector: software services (projects), and software product and research and development (products). Each of the above two divisions may be operating in Boxall's (2003) classification of Type 1 to 3 service markets.

Banerjee further suggests that the strategic focus of Indian IT organisations is to seek governance over their strategic milieu, where the strategic milieu is typical of a Marshallian (Marshall, 1920) market. Firms seek governance in a particular locality or domain, and in particular regional markets and resources through strategic alliances and strategic demand management. Firms' strategic composition and structure allows them to seek governance over other firms and customers operating in their strategic milieu. Indian IT firms, according to Banerjee, have a fluid structure, which is typified by joining different strategic business units with certain common strategic processes.

In this strategic make-up, Banerjee (2004) suggests that knowledge needs to be differentiated into three types: knowledge as factor of production, knowledge as a source of competitive advantage, which depends on how it is combined or recombined internally (initially Penrose, 1968, and then a central argument of resource theorists), and 
knowledge as something which co-evolves with structure and an organisation's strategy. Such knowledge is not a pure asset that can be procured from the market.

Such knowledge is strategic in nature because it focuses on how other knowledge is organised through the structure more than through content. In essence, it is a decision rule, and such a decision rule allows the firms to 'switch' from one market (e.g. product) to another (services) in response to its strategic needs. In other words, based on the two main divisions in India's IT sector (product environment and project or services environment), and depending on an organisation's strategic make-up and its milieu, its knowledge assets and the need for development of higher level competencies will vary. Further, an organisation's ability to switch from product to project environment and viceversa is its core competence and can be a source of its competitive advantage. The above switching competence is developed through investment in strategic knowledge as it determines the ease with which a firm can switch to and from a service or a product market.

Overall, the above approaches highlight the increasing importance that investments in training and HR strategy have on organisational performance. Strategic human resource management theorists view training as part of a firm's strategy to improve employee commitment and to manage its human resource flow (Fombrun et al., 1984; Heyes \& Stuart, 1996; Rainbird, 1994). Proponents of the "soft HRM" theory (Storey, 1989) have viewed training as part of the overall human resource policies to produce high performance outcomes (Walton, 1985; Kochan \& Dyer, 1993; Pfeffer, 1994). As well as the benefits presented by the HPWPs' school of thought, the limitations identified include the use of single informants in the studies, the cross-sectional nature of the studies and sporadic diffusion of these practices, despite their bold claims (Purcell, 1999).

\section{Empirical Evidence: Linkages between Business Strategy, Product/Service Strategy, HPWPs, HR strategy, work organisation and technology, and Training}

Business strategy. In the UK, some of the earlier attempts to explain the role of training and HRM in implementing strategic change were made almost two decades ago by Warwick scholars in the UK (Sparrow \& Pettigrew, 1985; Pettigrew et al., 1988). Pettigrew and his colleagues identified four sets of factors that variously drive and stabilise training. They emphasised the importance of distinguishing between driving 
(triggering) and stabilising factors. In their model, training is triggered by either technical or product market changes that create a gap in the skills requirements. Factors that trigger training are linked to an organisation's strategic needs and its internal labour market; factors that stabilise provision of training can be both internal (having a training champion, senior management commitment, budget allocation, trade unions and a training infrastructure) and external (availability of skills in the external and internal labour markets, government grants and subsidies, legislative requirements) to the firm. This model proposed that training be stabilised through a combination of factors that are both internal and external to the organisation. Changes to a firm's strategy have a profound impact on its skills requirements and as a consequence on its training provision.

In Australia, Hayton et al. (1996) built on the above work undertaken by Warwick scholars in the UK and developed a model that further classified the factors into three groups: training drivers, mediating factors and environmental factors. Using human capital and neo-human capital theory assumptions, and the impact of HRM strategy and HPWPs on training, they developed this model, initially by undertaking case studies of thirty organisations, ten each from three manufacturing industry sectors. The researchers found a range of factors drive training demand in these Australian enterprises (see their model in Figure 8). They found workplace reorganisation, investment in quality, new technology and enterprise size to be significant drivers of training. A further 12 case studies in different industry sectors and a survey of 1760 Australian worksites were undertaken by almost the same group of researchers (McIntyre et al., 1996; Smith \& Hayton, 1999).

The results confirmed their earlier findings (Hayton et al., 1996), demonstrating that demand for training is governed by three key elements: training drivers, which are factors that are internal to the firm; environmental factors, which are factors that govern an organisation's external and internal environment (for example, government regulation and competition); and mediating factors, such as enterprise size, workforce composition and training infrastructure (see Figure 8 for the full model).

Ridoutt et al. (2002) further developed the Hayton et al (1996) model and confirmed its robustness by suggesting that its flexibility allows researchers to include or exclude one or more variables, depending on the industry sector being researched. Overall, Smith and Hayton (1999) and Ridoutt et al. (2002) found the introduction of new products or process 
technologies, organisational change, and technological innovation to be significant drivers of training provision for most training activities.

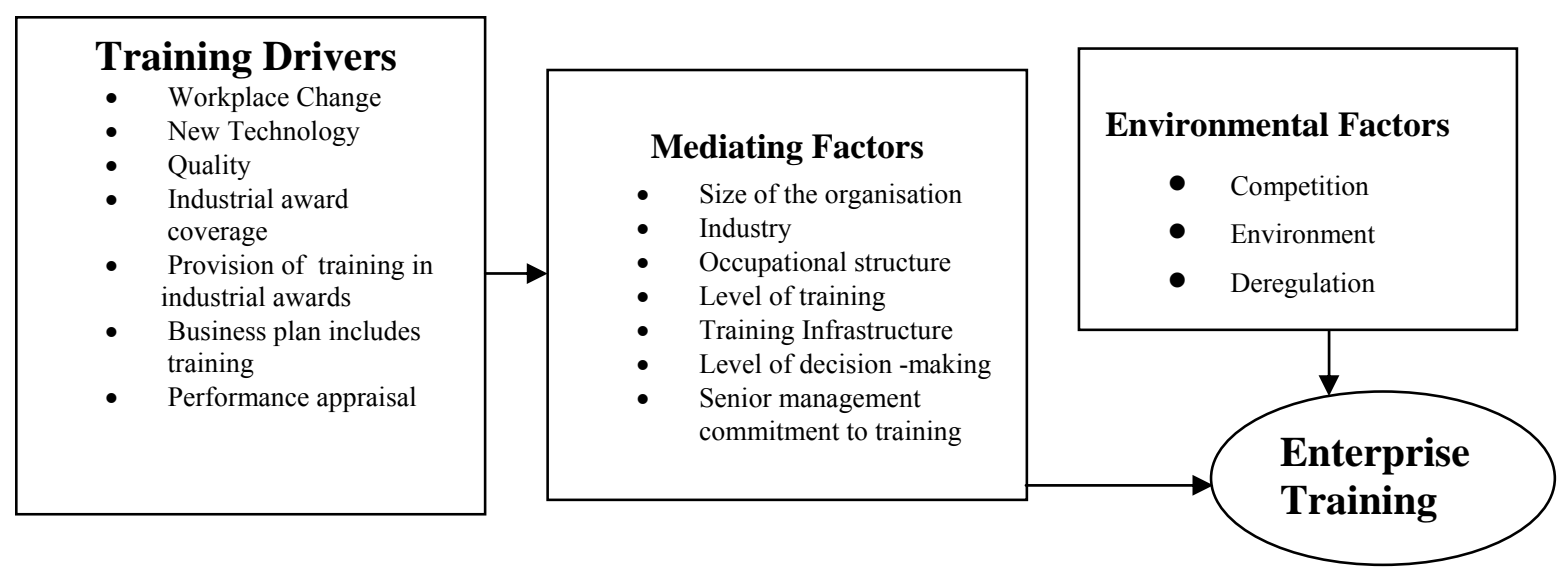

Figure 8. The Hayton et al. (1996) model.

(Adapted from Hayton et al., as cited in Ridoutt et al., 2002, p. 27).

In line with the UK and Australian attempts, researchers from Canada (Betcherman et al., 1997; Dostie \& Montmarquette, 2007) model certain factors in explaining the incidence of training provision in Canadian enterprises. They found support for a positive association between organisational change, business strategy, and the incidence of training.

Product specification strategy and training. Extending the research on training determinants, researchers from the National Institute of Social and Economic Research (NISER) and the Centre on Skills, Knowledge, and Organisational Performance (SKOPE) at Oxford and Warwick Universities in the UK have considered the role of product specification in determining the skills demand (Green et al., 2003; Mason, 2004, 2005). Green et al. and Mason (2004) found a positive association between the presence of high skill levels (evident through high qualification levels) and high product specification or high value-added production. The implication here is that highspecification products require high skills compared to low-specification products. Mason (2004) found strong support for high skills and an organisation's product strategy even after statistically controlling for enterprise size, site, sector, sales, and other variables. Green et al. (2003) and Ashton and Sung (2005) found demand for skills to be associated with technical change, whereas Kitchin and Blackburn (2002) found the introduction of new products or services to have a profound impact on skill demand. Overall, the above research points towards one common factor: organisation change-technical or structural— has a profound impact on skill formation. 
HPWPs and HR strategy. To understand the complexity of organisational change, Smith et al. $(2003,2004)$ undertook further research into five new management practices (NMPs) implemented in Australian enterprises and assessed their impact on training. Smith et al. $(2003,2004)$ found support for a positive association between various NMPs and indices of training. Smith et al.'s NMPs are similar to what has been described in the US and UK as high performance work practices (HPWPs) (Becker \& Huselid, 1998; Guest, 2000). Extant literature further offers a range of terminologies for such practices, for example, high commitment practices (Walton, 1985), high involvement work practices (Wood et al, 2001), high performance work organisation (HPWO) (Aston \& Sung, 2002; Lawler et al., 1998) and, more recently, specific bundles of sustainable HR and management practices (Purcell et al., 2003). Irrespective of the labels attached, these practices generally include total quality management (TQM), team working, learning organisation, business process re-engineering, lean production, job rotation, innovative HRM practices, and organisation-wide sharing of strategic and operational information. The underlying assumption is that, with increasing global competition and an economic slowdown, these HPWPs, when implemented together, will foster the development of higher level skills, which will consequently improve the performance of an enterprise and raise the overall satisfaction level of its customers and employees (Ashton \& Sung, 2002, 2005; Keep, 1999, 2000). Such an approach is often described as a "win-win" strategy for employees, employers and its stakeholders. Recent studies in the HPWPs tradition have also attempted to provide causal relationships between these practices and organisational performance (Guest et al., 2003; de Menezes \& Wood, 2006).

The heightened research interest in HPWPs as a vehicle for improving organisational performance has also drawn policy makers to consider their usefulness for developing national competitiveness and innovation in the UK (DIT, 2003; Keep, 1999, 2000). Some consider HPWPs as a vehicle for the high skills development needed in today's knowledge economy and have established linkages between HPWPs and demand for investment in skills (Ashton \& Sung, 2002; Felstead \& Ashton, 2000; Felstead \& Gallie, 2002). The recipe here again is a model of certain practices, which, if implemented, are likely to take organisations on a path of high skills development and consequently high performance and growth. Like most studies in this tradition (Osterman, 1994; Snell \& Dean, 1992), Smith et al. also found a move away from investment in technical and firmspecific skills to investment in generic and transferable skills because some of these management practices lend themselves more to the development of generic and 
transferable skills. Further, where the focus of such HPWPs or new management practices is on employee control and cost reduction, only technical skills are developed. However, where such HPWPs allow for empowerment, employee discretion, and autonomy, the focus of skills development includes both technical and behavioural skills (Ashton \& Sung, 2002; Smith et al., 2003; 2004).

Thus, where the focus of implementing new management practices is on increasing employee autonomy and discretion, firms are more likely to invest in general and technical training. Where the focus of such practices is on high employee control and low employee discretion, firms will invest in technical training (Proposition 4).

In a more recent consideration of drivers of training in the UK, Ashton and Sung (2006) offer a fresh approach by considering not only an organisation's competitive strategy (cost leadership and/or differentiation), but also the way its production function (interpersonal/social and technical systems) is organised. Ashton and Sung argue that an organisation's competitive strategy and its production function determine the nature and extent of skills demand. They differentiate the production function based on Wilkinson's classification (2002), wherein the technical system could be following either a massproduction or Fordist work organisation strategy or a differentiated work organisation strategy, characterised, typically, by adoption of HPWPs such as team working and lean production. Its social or interpersonal relations could either be highly task-focused or have a people development focus.

Through case studies of 10 UK organisations, Ashton and Sung found that firms operating with a mass production technical system strategy and having high task-focused interpersonal relations are less likely to invest in a broad range of skill sets. They found that firms having a differentiated production function or a non-Fordist work organisation, with a people development focus in their social and interpersonal work relations, are more likely to invest in a range of skills. However, this does not mean that "HPWOs are developing skills for the sake of skills"; instead, they found skills development to be closely linked to the firms' and individuals' performance outcomes and targets (Ashton \& Sung, 2005, p. 3).

In a HPWO, skills development serves two roles: first, to develop essential skills for the performance requirements of a job; second, to develop a motivational environment that helps employees to internalise learning so that they see learning as an integral part of 
their roles. The latter is similar to Purcell et al's (2003) finding of high organisational performance. Purcell et al., using the classic performance equation Performance $=f A M O$ (where performance is a function of $\mathrm{A}=$ Ability, $\mathrm{M}=$ Motivation, and $\mathrm{O}=$ Opportunity), argue that while basic skills and abilities are essential for performance, it is how the organisation motivates employees and provides opportunities for people to develop that creates superior organisational performance.

Work organisation, technology, and training. Post-Fordist writers (Piore \& Sabel, 1984) argue that the impact of technology on skills is positive as it enables firms to be flexible and responsive to market needs. Technological change requires employees to become skilled and conversant in new technology and adopt flexible specialisations in their production systems. Empirical evidence on both the deskilling and upskilling positions is mixed. Evidence from the US and the UK suggests that the process of deskilling and upskilling happens simultaneously in different industries and contexts (Cappelli, 1993; Gallie, 1991) Other studies identify an upskilling trend, wherein the demand for training increases with the introduction of technology (OECD/CERI, 1988; Smith \& Dowling, 2001; Smith \& Hayton, 1999).

Some authors have classified neo-Fordist work as modified or digital Taylorism in the context of the high technology skills needed in the 21st century (Brown et al., 2008; Taylor, 1998; Taylor \& Bain, 1999, 2001, 2004). Ritzer's (2004) revised work The McDonaldization of Society-Revised New Century Edition, is written in a similar vein. Ritzer demonstrates four pervasive dimensions of McDonaldisation: efficiency, calculability, predictability, and control through non-human technology. The range of industry sectors that have found application of this phenomenon has increased significantly from mass manufacturing facilities, through health care, education (Ritzer, 2004) and call centres (Taylor, 1998; Taylor \& Bain, 1999, 2001, 2004), to administrative work in the service sector (Baldry et al., 1998).

In the extant training demand models, the linkages between business strategy, HRM, HPWPs, and skills development were explored. Supporters of the HPWPs tradition (Ashton \& Sung, 2002) view implementation of HPWPs as a key to developing high skills. Critics of high involvement, high commitment HR practices, HPWPs, or new process technologies (Kumar, 2000; Lloyd \& Payne, 2004, 2005; Marchington \& Grugulis, 2000) argue against the diffusion of such practices on the basis that these 
practices have negative consequences for employees and their impact often leads to increased managerial control, work intensification, and labour alienation, despite having a positive impact on an organisation's surplus and productivity.

\section{Critique of Training Demand Models}

The limitations manifested by the factor models approach are as follows. The first refers to the implied causality and the linking of independent variables to certain dependent variables using sophisticated statistical techniques to demonstrate causality-like claims. The implications put forth by the researchers of such input-output models is that they are positing the presence of a causal relationship between certain independent variables and various measures and indices of training and that organisations can use such research to inform their training decisions (Smith \& Hayton, 1999; Hayton, Ridoutt et al, 2002; Kitchin \& Blackburn, 2002). Causation in the real world is too complex to be captured via a simplistic input-output model.

Secondly, the models have limited power to explain why certain training outcomes and variables are more significant in some firms than in others, even within the same industry. The models also fail to capture why the same levels of training outcomes are not noted in firms using the same NMPs or HPWPs. They also fail to explain why some firms that are operating in a low specification product/service continuum have a high demand for skills.

Lloyd and Payne (2004, 2005) suggest that the linkage between skills development and HPWPs is highly questionable and in some cases may even produce negative outcomes. They opine that researchers in the HPWPs tradition blame the low-product specification and low skills as the main reason for British enterprises' inability to compete in high specification markets. Diffusion of HPWPs is advanced as an alternative way of moving from a low skills Fordist work organisation to a "post-Fordist" form of work organisation that is flexible and requires the development of the high skills necessary for competing in high-product specification markets.

Lloyd and Payne (2004) believe that if that were to be the case, economic transformation would be a matter of moving towards these HPWPs. However, the empirical evidence is far from compelling and there is confusion over what constitutes HPWPs. Lloyd and Payne conclude by suggesting a need to develop a better job rather than focusing on the current approach, as the consequences of emulating a US-based vision could actually be 
harmful in the long run (Kumar, 2000; Marchington \& Grugulis, 2000). Third, the factor models approach is closed to the conceptualisation of training drivers from an external perspective, for example one that considers an organisation's clients/customers.

Notwithstanding the critics' view, empirical research undertaken in the HPWPs tradition has generally found a positive association between such practices and the nature and extent of training (Cappelli, 1993; Osterman, 1994; Smith \& Hayton, 1999; Smith et al., 2003, 2004). Further, investment in quality was found to be associated with most studies in the HPWPs tradition, although little is known as to why that is the case. Its role in provision of training needs further review and understanding. Boxall (2003) argues that the ability to invest in quality is dependent on an organisation's competitive strategy. It is more likely that firms operating in differentiated and highly differentiated product and services markets will invest in quality management systems. What follows from the above is that firms focusing on a cost leadership strategy will invest less in training and quality management systems (Proposition 5). A related proposition is that firms servicing slightly and highly differentiated service markets will invest more in training and quality management systems than firms servicing mass service markets (Proposition 6).

The following section explores the role of quality management in an organisation's production function and the relationship it has with training decision-making in greater detail.

\section{Quality Management and Training}

Total quality management (TQM) is generally defined as the presence of a quality management philosophy that focuses on customer satisfaction, continuous improvement and treating the organisation as a total system (Dean \& Snell, 1991; Sitkin et al., 1994; Snell \& Dean, 1992). Reed et al. (1996, 2000) have defined TQM content to include customer satisfaction focus, team working, cost reduction, continuous improvement, top leadership commitment, training and education, and having an appropriate work culture. For the purposes of this study, and similar to research undertaken in Australia (Prajogo \& McDermott, 2006), the common TQM content areas are encapsulated into three broad areas: an organisation's commitment to investment in quality and information sharing (leadership commitment and shared beliefs and values regarding systemic information sharing), its focus on continuous improvement (comprises internal and external customer focus and process management), and team working (functional teams and cross-functional 
integration) are considered in explicating its quality management approach. Additional themes, if any, about an organisation's approach to quality will be analysed during within- and cross-case analyses.

Quality management has its roots in the manufacturing sector, drawing upon disciplines such as economics, systems thinking, management, and psychology. With the increasing proportion of service work relative to manufacturing, however, its applications are now well established and considered highly relevant to a range of service sectors, including IT. Quality management practices have established an important place in today's organisational thinking and are found to improve organisational performance, and are seen as a source of sustained competitive advantage (Morgan \& Piercy, 1996; Reed et al., 2000). Reed et al. distinguish between TQM content and process and suggest that while the TQM content can be a source of competitive advantage to firms, it is how organisations deploy their TQM practices is what can potentially create sustained competitive advantage. Most quality management practices have a strong focus on statistical process control, minimising the variance from the norm, using a range of sophisticated statistical techniques for monitoring the quality of the product or service deliverable.

Although Porter (1985) has long argued that the quality of products and services is a primary basis for following a differentiation strategy, followers of the TQM approach advance its linkages with a cost leadership strategy. Some research points to the coexistence of the low cost and differentiation competitive strategies (Hill, 1988; Morgan \& Piercy, 1996). Hill (1988), rejects Porter's (1985) assertion that the two competitive strategies are fundamentally incompatible with each other and that a strategic choice is necessary to avoid being "stuck in the middle". Morgan and Piercy (1996) confirm the view that quality management approaches support both differentiation and cost leadership strategies. Prajogo (2007) found that the effect of differentiation strategy on quality was moderated by cost leadership strategy: the stronger the cost leadership strategy, the greater the effect.

Extant literature explains the links between product and service quality, cost leadership, and differentiation strategies (Belohlav, 1993; Prajogo, 2007). For example, Belohlav (1993) suggests that by achieving high quality, firms can pursue both differentiation and cost leadership strategies. By focusing on external dimensions of quality (customer 
satisfaction and innovation) differentiation can be achieved. Similarly, by focusing on internal aspects of quality (process improvement, waste reduction, cost reduction, and so on) low cost strategy can be serviced. Prajogo (2007) explains:

the synergy between external differentiation and internal cost leadership provides firms with flexibility to select the scheme of competition they want to pursue by charging premium price with high quality or offering lower price with relatively equal quality level to competitors.(p. 78)

The above distinction is critical to our understanding of how an integrated quality management approach can inform and implement functional integration between a firm's marketing, people, and operations management functions. Thus, firms in the IT sector can select quality management frameworks for delivering various strategic (cost leadership and differentiation) and operational (cost and waste reduction, functional integration, capability development, customer satisfaction, and process improvement) outcomes.

\section{Quality Management Approaches in the IT Sector}

ISO 9000, Carnegie Mellon University’s Capability Maturity Model (CMM) developed by its software engineering institute (SEI), Malcolm Baldrige National Quality Award (MBNQA), Six Sigma, and Lean Six Sigma are among the numerous quality management systems (QMS) adopted by firms in the IT sector. A closer analysis of the TQM, Six Sigma, Lean Production, ISO 9000 certification standards, and CMM certifications reveals that there is a programmed mandate for certain types of training for all employees involved, at the various stages of the quality approach adopted. Further, for organisations in the IT sector to remain competitive, they must continually develop and deliver quality products/services on time and at low cost. Their ability to deliver this is dependent on how the firm deploys the internal and external dimensions of the quality management approach. For example, high levels of process maturity as assessed against the CMM standard, a commonly used quality standard in ITSS organisations, is associated with high product quality and increased development effort (Harter et al., 2000). Harter et al. (2000) further found marginal benefits from sustained investment in quality on cycle time and development effort, thus suggesting an optimal level of investment in quality in certain types of software development approaches.

The initial intention behind the development of the CMM was to assess how able and robust are the software development processes used by contractors employed by the US 
Department of Defence. This model enabled the Government to assess contractors' capabilities such as software management, systems engineering, and project and risk management, at various stages of the software development lifecycle, similar to the Waterfall model, discussed in Chapter 2.

The model typically categorises process maturity at five levels. At Level 1, software development processes are characterised as ad hoc and chaotic. At Level 2, the processes are repeatable and can be done with some level of consistency, with well developed project management capabilities. At Level 3, the organisation defines its standards for each process with some best practice guidelines for projects, and has the ability to customise it to new projects. At Level 4, it develops detailed metrics for each standard and the management can effectively control the entire process by focusing on predictability and using advanced statistical process control techniques. Finally, at Level 5 , the organisation establishes quantified process improvement metrics for all processes in the organisation and engages in continual improvement and reviewing in light of the changing external conditions. The essence of the above approach is to first observe and capture the processes and then to simplify and standardise them, to avoid variance in its performance by developing metrics for such processes. Such a level of maturity occurs over a period of time through sustained investment in quality management resources, assessments and training (although, as Harter et al. (2000) found, over investment can be counter-productive).

Using Motorola as a case study, Belohlav (1993) illustrates how the use of Six Sigma methodologies can link to its differentiation strategy of offering high quality products and services. Six Sigma is a continuous process that focuses on reducing the error or defect rates to 3.45 per million opportunities of a process or a part. Belohlav (1993) argues that an average organisation operates at Sigma Level 4, which translates to 6210 defects per million opportunities. Sigma Level 5 translates to 233 defects per million opportunities. Where an organisation can focus on implanting this internal dimension of quality in its production function, the net results allow it to create a new competitive position in the market. Belohlav (1993) found in Motorola's case that a:

4 sigma manufacturer will spend in excess of $10 \%$ of the sales dollar on internal and external repair. A sigma 6 manufacturer will spend less than 1\%. A 4 sigma supplier cannot directly compete with a 6 sigma supplier and survive. (p. 62) 
The above approach is not just limited to manufacturing. Its proliferation is also high in India's ITSS and ITeS/BPO services environment, although the sigma and process maturity levels of firms would vary depending on the stage of their organisational and process lifecycle. Six Sigma can be applied for both internal process efficiencies (cost leadership), and external product/service performance (based on clients' perception and needs).

Parzinger (1997) emphasises that, for successful implementation of TQM, it is necessary to follow a stage-wise application of TQM through a product/process life cycle. Research suggests that higher levels of investment are required in quality processes and personnel training in the early stages of a product/service or process life cycle, and, in accordance with clients' expectations, with the expectation that productivity will improve (Krishnan et al., 2000). Doing so would explain some of the conflicting findings, for example, why in certain organisations TQM was successful and also had a positive impact on the formalisation of training, but was unsuccessful in others. This anomaly suggests the existence of a temporal dimension in the adoption and deployment of quality management systems and that it may have an impact on its training and production function. These considerations suggest that the strength of an organisation's quality management systems helps in shaping the nature and extent of training provision (Proposition 7).

\section{Empirical Evidence of the Impact of Quality Management Systems on Training}

In addition to mandated training in various TQM approaches, the extant training demand models also note its impact on provision of training. The presence of a well-developed TQM philosophy in an organisation requires not only data collection and analysis skills; it also requires those involved in the programme to develop behavioural skills in the areas of communication and teamwork, customer service, and knowledge of statistical techniques (Smith \& Hayton, 1999). To this end, Osterman (1995) and Smith et al. (2004) found that TQM has been the catalyst for firms to move from technical training to behavioural training.

In their study, Ridoutt et al. (2002) found a strong commitment to quality within the respondent population to be a driver for formalisation of training activities. However, limited influence was seen on the volume of the training. This finding was also evident in a study by Hayton et al. (1996): almost half of the surveyed enterprises (Hayton et al.) found quality to be an important driver of training and the single most important factor 
influencing training activity decision-making. It is difficult to explain the paradoxical findings: strong commitment to quality as an important driver on one hand and limited investment in training volumes on the other (Hayton et al., 1996; Ridoutt et al., 2002).

Ridoutt et al. (2002) offer two possible explanations. Firstly, different forms and levels of quality commitment result in different levels of training activity. Thus, it is important to measure different levels and forms of an organisation's commitment to quality. TQM exists on a continuum, from simple quality awareness, through specific quality initiatives, to fully fledged quality accreditation programmes and an integrated approach to quality. Secondly, there could be a link between quality and the broader concepts of the innovation process, the learning culture, the product or service life cycle, and organisational change. Russell (1999) identifies the tension that exists between the idea of quality as the establishment of predictable standards on the one hand and innovation and continuous improvement on the other. Russell (1999) argues that this tension can be resolved by explaining innovation as being at the sharp end of quality, where quality plays the role of continuously refining and improving the innovative ideas advanced.

Initially, organisations begin their commitment to quality through monitoring and auditing their processes. Later, as their commitment increases, they may adopt a more holistic approach, which includes innovation and change as facilitators of the entire process. The nature and extent of training will vary according to the nature and intensity of the innovation and its link with commitment to quality (Vogel, 2000). As organisations develop maturity for different processes at different stages of the product and/or service life cycle, varying training outcomes are achieved. Thus, the commitment to quality and its influence on training can be viewed as integral to the change process. It is for these reasons that quality becomes statistically less significant or is submerged under the more powerful effect of workplace change (Ridoutt et al., 2002).

\section{Quality Management in India's IT sector}

Commitment to quality has also increased significantly in Indian enterprises, especially after the Indian Government's liberalisation programme of the 1990s. A corporate drive to secure and retain quality accreditation programmes is evident in the high number of Indian organisations securing the ISO 9000 accreditation, and many more are actively adopting different variants of TQM. This drive has provided an additional impetus to the 
HRD function in the IT sector of India, where a growing number of organisations have adopted quality accreditation standards, such as ISO 9000 (Radice, 1995), the Software Engineering Institute's Capability Maturity Model (SEI-CMM) Levels 1-5 (Paulk et al., 1993), PCMM (People Capability Maturity Model), Six Sigma certifications, and the ITeS/BPO version of CMM. Most of these standards focus on aspects of process maturity and capability development, using training and development (Kumar, 2001; NASSCOM, 2005b). According to the Indian IT industry association NASSCOM, “As of December 2005, over 400 Indian companies had acquired quality certifications with 82 companies certified at SEI CMM Level 5 - higher than any other country in the world." (NASSCOM, 2006a, p. 2)

As noted in Chapter 2, quality has been used by domestic ITSS and ITeS/BPO firms for a number of purposes, ranging from increasing customer satisfaction, through process improvement, to market signalling effects, although there is limited empirical evidence to support such claims (Humphery et al., 1991; Dion, 1993). Only recently have we seen some empirical work on the effect of quality accreditation on firm performance in terms of increased revenues and higher price per unit of output (Arora \& Asundi, 2000). In a study of 95 Indian software firms, Arora and Asundi (2000) used surveys and site visits to interview more than 20 firms, including 12 of their US-based clients. The following themes emerged:

- Quality certification is an important signalling tool for potential customers.

- It provides the software vendors with the ability to deliver sophisticated and higher value-added services with a higher per unit output cost, thereby enabling them to undertake complex projects.

- It enables better understanding of clients' needs during the negotiations and even before the contracts are signed.

Further, for organisations to survive in the IT sector, they must continually develop and deliver products/services on time and at low cost. Their ability to do so depends on high levels of process maturity as assessed by the SEI-CMM standard, a commonly used quality standard in ITSS organisations, which is associated with high product quality and increased development effort (Harter et al., 2000). While the training demand models deepen our understanding of some key factors that influence training decision-making and 
their relationships with each other, the input-process-output approach adopted by the above models has limitations and needs further probing.

Irrespective of whether an organisation operates in a mass production, differentiated or slightly differentiated market segments, an important consideration for its success lies in its ability to satisfy its customers and be able to adapt fast to the changing environment, a consideration that has thus far not been addressed in the HRD literature. Further, in the evolution and growth of certain large IT organisations in India, among other capabilities, the development of client-specific capabilities was noted as one of the key reasons for their sustained growth and performance. (Refer to Chapter 2.)

However, little is known in a developing country context how this capability is developed in differentiated or mass markets and how an organisation's social and technical relation systems drive or hinder the development of such capabilities. Extant literature suggests that for an organisation to develop distinctive capabilities, it should demonstrate a commitment to developing a learning culture, open-mindedness for new learning, and must develop a shared vision (Senge, 1990). Further, in the context of globalisation and outsourcing, there is a high level of interdependence and dynamic coordination in knowledge transfer from the client to the service provider (Banerjee, 2004). This requires an understanding of how organisations develop client-specific capabilities and other learning necessary to meet their clients' needs. Therefore, on the assumption that organisational values drive behaviours and actions, the following section discusses the market-based organisational learning framework developed by Sinkula et al. (1997).

\section{Market-Based Organisational Learning Framework}

Organisations that are able to learn and transform themselves in response to the dynamic external environment are more likely to sustain themselves in the long term. Organisational learning depicts a cognitive view of the organisation and has often been noted as one of the key ways to achieve competitive advantage and be responsive to changes in its environment (Dickson, 1996; Hunt \& Morgan, 1996). Although numerous organisational learning processes have been advanced in the literature (Deshpande \& Webster, 1989; de Guess, 1988; Huber, 1991; Sinkula, 1994), some consensus exists regarding the focus of organisational learning. In short, involves the acquisition and 
development of new knowledge or insights that can potentially change an organisation's behaviour towards its strategic and operational milieus.

Organisational learning is based on the assumption that learning facilitates behavioural change that subsequently results in better organisational performance. To achieve highlevel performance, organisations need to develop a learning culture that values investment in the training and development of employees, to build up systems that support the creation and acquisition of new knowledge, and to implement these systems at organisational, group, and individual levels.

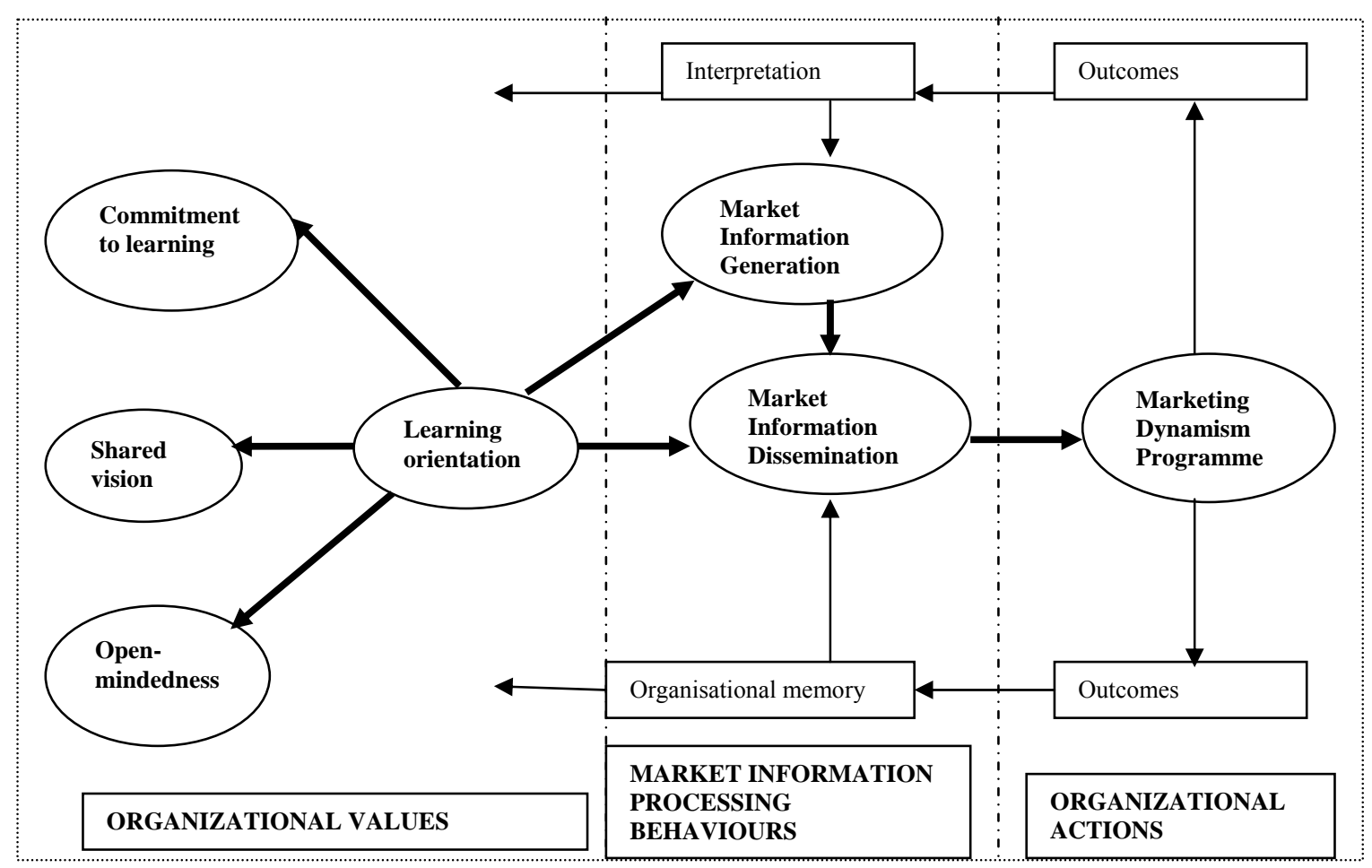

Figure 9. A framework for market-based organisational learning.

(Adapted from Sinkula et al., 1997, p. 307)

In their conceptualisation of a market-based organisational learning process, Sinkula et al. (1997) suggest that the process of organisational learning is facilitated by three elements: organisational values that promote learning, an organisation's market-information processing behaviours, and organisational actions (see Figure 9 for details). Owing to the "tacit, covert, and unobservable" nature of interpretation and organisational memory, Sinkula et al. (1997:308) excluded these constructs from their empirical model. Before the framework is explicated, the two main constructs-an organisation's learning orientation and market information processing behaviours - are explained below. 
It has been defined in different contexts but, in general, it involves three sets of organisational values associated with an organisation's tendency to learn. These values are commitment to learning, open-mindedness, and developing a shared vision to influence a firm's ability to create and use knowledge (Argyris \& Schon, 1978; Senge, 1990; Garvin, 1993). Commitment to learning is an organisational value that fosters learning through ongoing training and development of its employees and allocates resources for such development. Open-mindedness requires an organisation to challenge its current theory-in-use and any new information it processes from internal and external sources. Shared vision encompasses its ability to communicate and disseminate its theoryin-use and any new knowledge and competencies that it has developed throughout the organisation. These values can be implemented partly by direct investment in training and partly by fostering a culture of supporting and sharing new learning and skills development at all levels.

Learning orientation influences the degree to which an organisation is satisfied with its theory-in-use and, hence, the degree to which proactive learning occurs (Senge 1990; Sinkula et al., 1997). An organisation's learning orientation could be adaptive (single loop), generative (double loop), or transformative (triple loop), which has an impact on its ability to replace its existing theory-in-use. Single loop (Argyris \& Schon, 1978) or adaptive learning (Senge, 1990) is mostly about an organisation's dominant logic and management approaches to business problems. It is achieved by extending its theory-inuse through strong management systems and processes. Organisational changes are usually sequential and incremental in nature (Slater \& Narver, 1995). Generative or double-loop learning (Argyris \& Schon, 1978; Senge, 1990) is indicative of an organisation's ability to unlearn its current ways of doing things to improve its processes. It is possible through an organisation's open-mindedness and how it critically evaluates its existing theory from time to time. Such learning is essential for continuous improvement and breakthrough innovations in its products, services and processes (Baker \& Sinkula, 1999). Sinkula et al. (1997) refer to such learning orientation as knowledge-questioning values. Triple loop or transformational learning is evident when an organisation undergoes a major paradigm shift, such as when a manufacturing organisation reinvents itself as a service or a logistics operation. In the context of this research, an organisation's ability to switch from a service to a product environment or from a captive to a third-party and vice-versa is typical of such learning transformation. 


\section{Market Information Processing (MIP)}

To understand the influence of client needs on the creation of new knowledge, which is a critical input for any training provision, we need to understand the concept of market information processing. Market information processing is the process by which external market information is transformed into knowledge (Sinkula, 1994). It involves sensing and disseminating of information from the external market across the organisation. Information dissemination is the ability to diffuse the information generated, vertically and horizontally (Argyris \& Schon, 1978). Of more importance is information sensing or generation because it involves capturing precise and critical information about a customer's needs and the external competitive environment. If information acquisition is done well, disseminating it across the relevant parts of the organisation and then framing a response is relatively easy.

Market information processing is developed from a key marketing construct, market orientation (MO). While the concept of MO was developed with the intent of meeting an organisation's strategic marketing needs, the information thus acquired and disseminated has direct relevance for developing various operational, marketing, and training responses. From a client perspective, market orientation's linkage with learning orientation (Sinkula et al., 1997) is critical in strengthening its relationship with an organisation's training and development efforts and marketing outcomes. Kohli et al. (1993) developed a measure for MO, MARKOR, and defined it as: "the organizationwide generation of market intelligence pertaining to current and future needs of customers, dissemination of information horizontally and vertically within the organization, and the organizationwide action or responsiveness to market intelligence.” (p. 467)

Narver and Slater (1990) contend that it is possible to achieve sustained competitive advantage by providing superior value to one's customers. It is possible by creating an organisational culture that supports behaviours for creating superior customer value. Market orientation is one such knowledge-producing behaviour (Sinkula et al., 1997). It consists of three behavioural components: information sensing or acquisition from an organisation's customers and competitors, horizontal and vertical dissemination of such information within the organisation, and framing appropriate organisational responses in relation to such information via inter-functional coordination (Kohli et al., 1993; Narver \& Slater, 1990). Information generation ability is critical as it enables the organisation to keep itself informed about its customers and competitors. However, if its information 
dissemination and response-framing ability are inefficient and ineffective, changes to its theory-in-use will be limited. An organisation's ability to store, process, and interpet new information enhances or hinders the way in which such information is attended to before any response is framed.

\section{Key Relationships}

Sinkula et al. (1997) were the first to test the interrelationships between organisational learning, a values-based construct, which formally connects organisational values (Argyris \& Schon, 1978; Fiol \& Lyles, 1985; Senge, 1990) to an organisation's market information processing behaviours (Kohli et al., 1993; Narver \& Slater, 1990), and organisational actions. On the assumption that values drive behaviours and subsequent actions, Sinkula et al. (1997) tested three hypotheses:

1. The extent to which an organisation engages in market information behaviour is a function of its learning orientation.

2. An organisation's dissemination of market information will be a direct and indirect function of its learning orientation, such that the indirect effect of learning orientation on information dissemination is dependent on its information generation.

3. The frequency with which an organisation disseminates its marketing programmes (organisational response) will be a function of the extent to which it disseminates the information.

\section{Empirical Evidence of Key Relationships}

Sinkula et al. (1997) found that a more positive learning orientation (a values-based construct) will lead to increased market information generation and dissemination (knowledge-based construct), which, in turn, affects the degree to which an organisation makes changes to its strategies (a behavioural construct). Organisations with high levels of learning orientation (LO) are more likely to question their current theory-in-use, challenge basic assumptions and be open to new ideas and knowledge. While new knowledge is procured through an organisation's market orientation (MO) abilities, it can be refined, redefined and challenged through its LO, depending on the extent to which its LO and MO are developed. Organisations may thus demonstrate, adaptive, generative, or transformational learning processes. Strong LO induces knowledge questioning values to refine the information acquired and disseminated through a firm's MO. If a firm has high 
MO and medium to low LO, it is likely to result in adaptive learning (Slater \& Narver, 1995) rather than generative learning, which is more likely to be the type of learning that occurs when an organisation has high LO and high MO (Argyris \& Schon, 1978; Senge, 1990; Sinkula, 1994). In other words, high LO is likely to enhance the market-orientated behaviours because a strong learning orientation constantly challenges the assumptions of the information and an organisation's theory-in-use.

In terms of adaptability of a new product or service, it is likely that strong market orientation will result in faster product development, especially if the learning orientation is low. Strong learning orientation may not necessarily result in new product development because it challenges the clients' assumptions, and it relies on a range of additional information sources, including market intelligence processing, to make informed decisions, which may have an impact on the decision to develop what customers want. As a result, firms with low LO and high MO are more likely to focus on product, service and process enhancements or incremental changes rather than on new products or radical innovations (Baker \& Sinkula, 1999, 2002, 2005). In a framework designed to enable the understanding of the relationships between market orientation, learning orientation and product innovation, Baker and Sinkula (2002) explicate a hierarchy of five levels of organisational learning and three types of marketing firms. The learning hierarchy, starting from low to high is as follows: conditioning, modelling, adaptive learning, generative learning, and meta-learning. The three groups of marketing firms are firms with strong market and learning orientations, firms with strong market orientation and weak learning orientation, and firms with weak learning and market orientations.

Baker and Sinkula (2002) advanced numerous propositions from this framework. They proposed that firms in the first group define and lead the environment they are in and are more likely to demonstrate meta- and generative learning, which is typical of market leaders and firms that are radical innovators. Firms in the second group are responsive to the environment and more likely to demonstrate adaptive learning, and market-driven incremental innovation. Firms in the last group are more likely to demonstrate managerdriven incremental innovation, and learning is typical of imitative behaviour best learned in environments that support modelling and conditioning learning. They undertook subsequent research (Baker \& Sinkula, 2005, 2007) to test their framework and reaffirmed the positive role high market orientation plays in developing incremental and radical innovation. From the above, it can be proposed that in a high-tech services 
environment, high levels of market and learning orientation will require higher levels of investment in technical and general skills training (Proposition 8).

In Australia studying the impact of various new management practices on training, Smith et al. (2004) measured the impact of learning orientation amongst five other management practices. Using learning orientation measures employed by Sinkula et al. (1997), Smith et al. found learning orientation to be highly correlated with a number of training factors, such as increased training expenditure, proportionately greater attention to the training of managers, increased focus on development of behavioural skills, greater use of coaching and mentoring, and increased decentralisation of responsibility for training to line managers.

In recent years, this framework has attracted a lot of interest and has attempted to link an organisation's learning and market orientation to a range of organisational performance outcomes, such as increased market share, customer satisfaction, innovation, new product success, profitability, efficiency, and a better return on its marketing communications programme (Baker \& Sinkula, 1999a, b; 2002, 2005, 2007; Cano et al., 2004; Narver et al., 2004; Stewart \& Mavondo, 2005).

Cano et al. (2004) undertook a meta-analysis of the relationship between market orientation and business performance from five continents. Cano et al. tested the relationship between a range of contextual and moderating factors, such as national culture, profit versus non-profit firms, and services versus manufacturing firms. They found a strong relationship between market orientation and business performance in service and not-for-profit firms. Such a relationship is understandable as service firms have a closer interaction with their customers or clients relative to manufacturing and forprofit firms, which typically operate in a product environment and rely on other marketing and resource maximisation strategies for increasing their business performance. There was no significant difference noted between individualistic and collectivist cultures, suggesting that the relationship is not culture-specific. The above findings are relevant to this study because the study covers both aspects of industry type in India's IT sector: IT software services and business process outsourcing project firms (services) and firms in software product development, and those offering customised products for ITeS/BPO (such an environment can be seen as a proxy to the product (manufacturing) paradigm). 
Despite the above claims, there is limited understanding of how MO and LO capabilities can be developed. Even though customer satisfaction is one of the main focus areas of TQM and marketing, there is a paucity of empirical research that considers the role TQM plays in enhancing MO capabilities (Day, 1994). Day (1994) makes a strong case for utilising the tools available under the umbrella of TQM for implementing strong MO change programmes. Similarly, although both LO and TQM focus on challenging the status quo through open-mindedness and continuous improvement respectively, there are limited attempts to explore the relationships between the two concepts. Further, as quality management strategy can serve both differentiation and cost leadership strategies, understanding its impact is critical to the concept of $\mathrm{MO}$ and the market-based organisational learning framework.

\section{Summary of the Review, Resultant Gaps, and the Study's Research Questions}

This chapter first provided a review of the theoretical bases of HRD in general and various training demand models in particular. To this end, the review considered a number of theories that advance our understanding of why firms invest in training. The skill drivers noted in major research studies and theories are summarised in Table 4a. In essence, HRD's performance and learning paradigms are evident in the preceding review of literature. In meeting the above outcomes of training, a number of organisational, individual, external and regulatory factors have emerged from the literature. A list of the key training factors is provided in Table $4 \mathrm{a}$.

From the review, the main rationales for investing in training (and their respective theoretical orientations) included:

- enhancing individual and organisational performance/productivity (human capital theory)

- improving an organisation's ability to cope and implement technological changes and managerial innovations for improving organisational productivity (neo-human capital theories)

- managing career aspirations and gaining employee commitment through investment in training (HRM models) 
Table 4a.

Theoretical Foundations of Main Factors Influencing Investment in Training: Focus and Level of Analysis

\begin{tabular}{|c|c|c|c|c|}
\hline $\begin{array}{l}\text { Theoretical foundations } \\
\text { /orientations }\end{array}$ & $\begin{array}{l}\text { Main factors that influence } \\
\text { investment in training }\end{array}$ & Focus and level of analysis & Sectors studied & Theorist(s) \\
\hline Human Capital Theory & Firm's productivity & $\begin{array}{l}\text { Supply side of skills: invest } \\
\text { in individual level education } \\
\text { and training }\end{array}$ & $\begin{array}{l}\text { Mostly manufacturing } \\
\text { sector }\end{array}$ & $\begin{array}{l}\text { (Becker, 1964; Mincer, 1974; } \\
\text { Strober, 1990) }\end{array}$ \\
\hline $\begin{array}{l}\text { Neo-human capital } \\
\text { theories }\end{array}$ & $\begin{array}{l}\text { 1. Firm's productivity } \\
\text { 2. Technological change } \\
\text { 3. Strategic HRM } \\
\text { 4. Product changes } \\
\text { 5. Process changes } \\
\text { 6. Asymmetric information } \\
\text { about the employment market }\end{array}$ & $\begin{array}{l}\text { Supply and demand side of } \\
\text { skills. Individual and } \\
\text { organisational level. Policy } \\
\text { influence. Comparative } \\
\text { advantage of educated } \\
\text { workers over less educated. } \\
\text { Technological change and } \\
\text { external labour market }\end{array}$ & $\begin{array}{l}\text { Manufacturing and services } \\
\text { sectors }\end{array}$ & $\begin{array}{l}\text { (Wozniak, 1984, 1987; Bartel \& } \\
\text { Lichtenberg, 1987; OECD/CERI, } \\
\text { 1986; 1988; Acemoglou \& } \\
\text { Pischke, 1999a, 1999b; Pettigrew } \\
\text { et al., 1988; Sparrow \& } \\
\text { Pettigrew, 1985; Hayton et al., } \\
\text { 1996; Smith \& Hayton, 1999; } \\
\text { Smith, 1997; Smith \& Dowling, } \\
\text { 2001; Ridoutt et al., 2002; Dawe, } \\
\text { 2003) }\end{array}$ \\
\hline Game theory & $\begin{array}{l}\text { Macro-level focus on the role of } \\
\text { institutional dynamics. }\end{array}$ & $\begin{array}{l}\text { Individual, organisational } \\
\text { and government }\end{array}$ & Mostly manufacturing & (Finegold, 1991, 1992) \\
\hline $\begin{array}{l}\text { Studies using multiple } \\
\text { theoretical orientations } \\
\text { (Human capital, neo- } \\
\text { human capital theories, } \\
\text { HRM, and HPWPs) }\end{array}$ & $\begin{array}{l}\text { 1.Business and competitive } \\
\text { strategy } \\
\text { 2. Organisational performance } \\
\text { 3. Technological \& } \\
\text { organisational change } \\
\text { 4. Size } \\
\text { 5. Industry sector } \\
\text { 6. Quality } \\
\text { 7. Trade unions } \\
\text { 8. Regulatory changes } \\
\text { 9. NMPs/HPWPs } \\
\text { 10. Work organisation and } \\
\text { production systems } \\
\text { 11. Product and service } \\
\text { specification } \\
\text { 12. SHRM practices }\end{array}$ & $\begin{array}{l}\text { Industry, organisational, and } \\
\text { individual level. regulatory } \\
\text { and external environmental } \\
\text { factors }\end{array}$ & $\begin{array}{l}\text { Manufacturing and service } \\
\text { sectors }\end{array}$ & $\begin{array}{l}\text { (Ashton \& Sung, 2002; 2005; } \\
\text { 2006; Green et al., 2003; Mason, } \\
\text { 2004; 2005; Hayton et al.,1996; } \\
\text { Smith \& Hayton, 1999; Smith, } \\
\text { 1997; Piore \& Sabel, 1984; } \\
\text { Ridoutt et al., 2002; Dawe 2003; } \\
\text { Smith et al., 2004; Betcherman et } \\
\text { al., 1997; Dostie \& } \\
\text { Montmarquette, 2007) }\end{array}$ \\
\hline
\end{tabular}

- improving the quality of service delivery, client satisfaction and cost reduction (various quality management approaches)

- developing a high-skills society by implementing HPWPs (HPWPs and HRM theories)

- servicing an organisation's business strategy through appropriate product and services strategies (Strategy and HRM). 
Also evident from the above review is the limited number of studies researching the services sector, especially high technology services such as information technology services. Further, the extant literature is scant on this aspect of training, especially within the Indian context. Thus, this thesis will be looking at factors that influence a firm's decision to invest in training in the IT sector in the context of a developing country. The first research question this thesis aims to explore addresses this gap in the literature.

\section{Q1. What are the main factors that influence a firm's decision to invest in training in IT sector, within the Indian context?}

Further, the field of HRD has considered research mainly from a firm's internal perspective. HRD literature highlights the need to study the much-neglected and oftencited aspect of considering training decision making from an outward-looking perspective, especially one that involves its clients/customers (Leimbach \& Baldwin, 1997; Bing et al., 2003; Short, 2006). An organisation's clients and customers are particularly important given the distinct features of the IT-enabled services industry, such as the high level of interdependence between the service provider and the client firm, and the dynamic nature of the environment (Banerjee, 2004), which forms the basis for answering the second research question of this thesis. To this end, the second question aims to explore this gap in literature.

\section{Q2. Do an organisation's clients/customers have an impact on investment in training? If so, why and how do they influence it?}

Although the nature of studies undertaken in the quantitative tradition offer us some insights into the relationships between factors, Smith (2000) argues for the need to develop a deeper understanding of the relationships considering the complex nature of organisational training decision-making. The complex and dynamic nature of coordination between a client and service provider firm in a dynamic outsourcing environment (Banerjee, 2004) in the context of a developing country such as India calls for a better understanding of the interaction between various internal and external factors. This need forms the basis of the third question this thesis is attempting to answer.

\section{Q3. How do various factors-internal and external to the firm-interact with one another in the provision of training in India's IT sector?}

Some of the major critiques of the extant training demand models focus on the limited explanatory power these models have with regard to variation in levels of training. 
Similar sets of HPWPs yield differing levels of training outcomes for firms in the same industry sector. A detailed understanding of contextual and cultural factors is critical to further our understanding of why firms offer varying levels of training. Answering the study's above three questions would enable some insights to be drawn and interpretations to be made about the variations in training. The last question of the thesis aims to address this gap in the literature.

\section{Q4. Why do some firms invest more than others even if they are in the same industry sector?}

Table $4 \mathrm{~b}$ below presents a summary of the study's key propositions and how these relate to the above research questions.

Table 4b.

Study's Propositions and its relationship to the research questions

\begin{tabular}{|l|l|}
\hline \multicolumn{1}{|c|}{ Propositions } & \multicolumn{1}{|c|}{$\begin{array}{c}\text { Research } \\
\text { Question }\end{array}$} \\
\hline $\begin{array}{l}\text { Proposition 1: Firms will invest more in firm-specific training than general } \\
\text { skills training. }\end{array}$ & 3 and 4 \\
\hline $\begin{array}{l}\text { Proposition 2: Firms will invest in general skills training due to } \\
\text { asymmetrical market information about their new employees. }\end{array}$ & 1,3 , and 4 \\
\hline $\begin{array}{l}\text { Proposition 3: The demand for highly skilled workers will decline with } \\
\text { experience on a given technology. }\end{array}$ & $1,2,3$, and 4 \\
\hline $\begin{array}{l}\text { Proposition 4: Where the focus of implementing new management practices } \\
\text { is on increasing employee autonomy and discretion, firms are more likely to } \\
\text { invest in general and technical training. Where the focus of such practices } \\
\text { is on high employee control and low employee discretion, firms will invest } \\
\text { in technical training. }\end{array}$ & 3 and 4 \\
\hline $\begin{array}{l}\text { Proposition 5: Firms focusing on a cost leadership strategy will invest less } \\
\text { in training and quality management systems. }\end{array}$ & 1,3 , and 4 \\
\hline $\begin{array}{l}\text { Proposition 6: Firms servicing slightly and highly differentiated service } \\
\text { markets will invest more in training and quality management systems than } \\
\text { firms servicing mass service markets. }\end{array}$ & $1,2,3$, and 4 \\
\hline $\begin{array}{l}\text { Proposition 7: The strength of an organisation's quality management } \\
\text { systems helps in shaping the nature and extent of training provision. }\end{array}$ & 1,3 , and 4 \\
\hline $\begin{array}{l}\text { Proposition 8: In high-tech services environment, high levels of market and } \\
\text { learning orientation will require higher levels of investment in technical } \\
\text { and general skills training. }\end{array}$ & 2 and 3 \\
\hline
\end{tabular}




\section{CONCLUSION}

This chapter has provided a detailed literature review and critique of the dominant theories, frameworks, and studies that explicate a firm's decision to invest in training. The studies included both the supply of, and demand for, skills and training in organisations. Following a detailed review, the gaps in the literature were identified and the unresolved issues in the literature formed the basis of this study's research questions. Finally, this chapter provided theoretical perspectives, which inform and guide the research design, data collection, analysis, and help situate the findings in relevant literature and theories. 


\section{CHAPTER 4}

\section{METHODOLOGY}

\section{Introduction}

The purpose of this chapter is to explain the methodological approach followed in this research, and to show how the methodology adopted fits in with the research questions that form the basis of this study. The nature of this research is exploratory and descriptive, and it aims to extend the ongoing theory-building attempts over the last two decades regarding the factors that influence a firm's decision to invest in training. The details of such factors and extant theoretical perspectives were provided in Chapter 3.

This thesis employs a multiple case-study design strategy and analyses primarily qualitative data from the case study organisations to seek a better understanding of the factors that influence a firm's decision to invest in training in the context of India's IT sector. In line with the unresolved issues in the literature and the study's ensuing research questions, this thesis also considers the role of an organisation's clients in shaping the nature and extent of training provision. It also aims to explain the variation in training resulting from the complex interaction between various factors. Given that a related focus of research is to extend the theory-building attempts and to develop a better understanding of key factors that influence training, Eisenhardt's (1989) suggestion of adopting an inductive approach is appropriate.

The first section of this chapter explains the rationale for the qualitative approach and an explanation of the methodological appropriateness and congruence in the study's research design (Morse \& Richards, 2007). The second section provides the rationale for adopting a case study research method (Eisenhardt, 1989; Yin, 2003). This thesis adopts a qualitative case study approach to understanding the complexities in organisation decision making with regard to training. Details about the rationale and the steps outlined in the method are provided. These include:

- the design of the multi-case study research 
- the conduct of the research, including data collection techniques, such as semistructured interviews, organisational records, documents, and non-participant observation

- the analytical strategies and techniques employed

- reporting of case findings.

\section{Rationale for a Qualitative Case Study Research Design}

An under-developed state of theory in the phenomenon of interest, a relatively new industry, a developing country context, and the complex and broad nature of organisational decision-making makes qualitative methodology an appropriate approach (Creswell, 2007; Lincoln \& Guba, 2000; Patton, 2002). Further reasons for using qualitative methodology include: to develop a better understanding of the problem in its contextual environment; to overcome the difficulties of inappropriate measures employed by quantitative studies (because they sometimes do not fit the problem at hand); to maintain openness to new categories of data emerging during the analysis; and to allow for flexibility in data collection.

Morse and Richards (2007) argue for methodological purposiveness and congruence. They suggest that a study's research questions should lead the researcher towards methods of collecting data and towards employing certain analytical strategies. What the researcher asks, where the data is collected from, and how the data is analysed and conclusions are drawn should all fit together. Purposiveness arises from the nature of the research questions and the type of data to be collected. Congruence is about the fit between: the research problem and the research questions; the research questions and the method; and the chosen method and the data handling and analysis techniques employed.

In applying the above view of methodological purposiveness to this study, it is evident from the preceding chapter that the state of theory in the area of enterprise training is relatively underdeveloped. Despite numerous theory-building attempts, research suggests that there is significant variation in an organisation's decision making about training (Ashton \& Sung, 2006; Lloyd \& Payne, 2004, 2005). The current theoretical perspectives, although useful, are found lacking in explaining the variance in training provision. Thus, a better understanding of an organisation's unique and varied cultural context is needed. It can be achieved by researching the phenomenon in its natural environment. Moreover, the 
measures employed by most training demand models have been taken from developed countries and applied to firms operating in a manufacturing sector.

On undertaking initial discussions with 30 human resource (HR) and training managers of IT organisations in India, to explore the usability of measures of training and a range of factors from the extant literature, the researcher found these measures to be inappropriate for India's cultural, and the sector's unique contextual, environment. The discussions further suggested that the data to be collected cannot be provided by a single informant. It requires elicitation of information from multiple informants. Further, the terminologies of certain concepts from the extant literature were not well-understood by the HR and training managers.

The impact of clients' needs on an organisation's decision to invest in training, a muchcited and often-neglected area in the HRD literature, can be better understood by studying organisations in their real-life context, because the sector is relatively new and literature on this aspect is not well developed. A qualitative approach generates data that is unique to a site and provides a contextual understanding of the complexity of training decisionmaking. Understanding different contexts and the interdependence between the organisation and the client firms - which is typical of this sector-is crucial to our understanding of an organisation's training decision making processes.

In terms of methodological congruence-the fit between the research problem, the study's questions and the method employed — data collected and analysis techniques used are explicated in the following section. In applying the above approach to this thesis, the researcher adopts a critical view of the extant training demand models and theoretical perspectives covered in Chapter 3, and considers that they offer only an approximate understanding of the phenomenon. Information obtained through studying the organisations in their real-life and contextual surroundings is believed to offer a better understanding of the phenomenon. In a similar vein, Flyvberg (2006) emphasises on the need to undertake rich and descriptive context-dependent case study research for advancing the state of theory-building in emerging areas of research.

Given the above rationale, the study's research questions and the theory-building approach adopted here, a qualitative case study design is considered an appropriate research strategy (Eisenhardt, 1989; Yin, 2003). The following section explains the 
rationale for this approach, outlines the key steps followed in generation of new understanding, and demonstrates methodological congruence as applied to the study's research design.

\section{Rationale for Case Study Research}

In the last two decades, case studies have found application in a range of applied disciplines, such as information systems (Benbasat et al., 1987; Lee, 1989; Dube \& Pare; 2003), human resource management (Dyer, 1984; Edwards, 1995), and HRD (Ashton \& Sung, 2006; Dooley, 2002; Smith \& Dowling, 2001; Smith, 2000). Although the terms case study, case study research and theory-building using case study research have been acknowledged as representing established and legitimate research strategies (Dooley, 2002), there appears to be some confusion over the use of the three terms. They should not be used interchangeably. Dooley (2002) suggests a "case itself is an account of an activity, event, or problem. The case usually describes a series of events that reflect the activity or problem as it happened" (p. 337). Yin (2003) defines a case study as "an empirical inquiry that investigates a contemporary phenomenon within its real-life context, especially when the boundaries between the phenomenon and context are not clearly evident" (p. 13) and specifies the nature of research questions posed.

If the research focuses on the how and why questions-where the researcher has no control over the behavioural events - and the study focuses on contemporary events, case study research is an appropriate research strategy (Yin, 2003). Further, the what questions form a legitimate rationale for exploratory case studies. This study fulfils all the above established criteria for undertaking case study research. Moreover, as Smith (2000) suggests, the under-developed theory in the area of enterprise training renders exploratory case study research an appropriate research strategy. In addition, organisational-level and rich contextual data collected through case studies is useful in informing the theory building in a given field (Yin, 2003; Miles \& Huberman, 1984; Eisenhardt, 1989). Case studies then focus on understanding a limited number of events or situations and the nature of relationships between them in a unique contextual setting.

Although Yin (2003) and Eisenhardt (1989) outline distinct steps in case study research and in using case studies in theory building, Dooley (2002) argues, "There appears to be no clarity on the role case study research plays within the process of theory building" ( $p$. 
346). A general method of theory building involves five key phases (see Figure 10 for details): conceptual development, operationalisation, confirmation or disconfirmation, application, and continuous refinement and development.

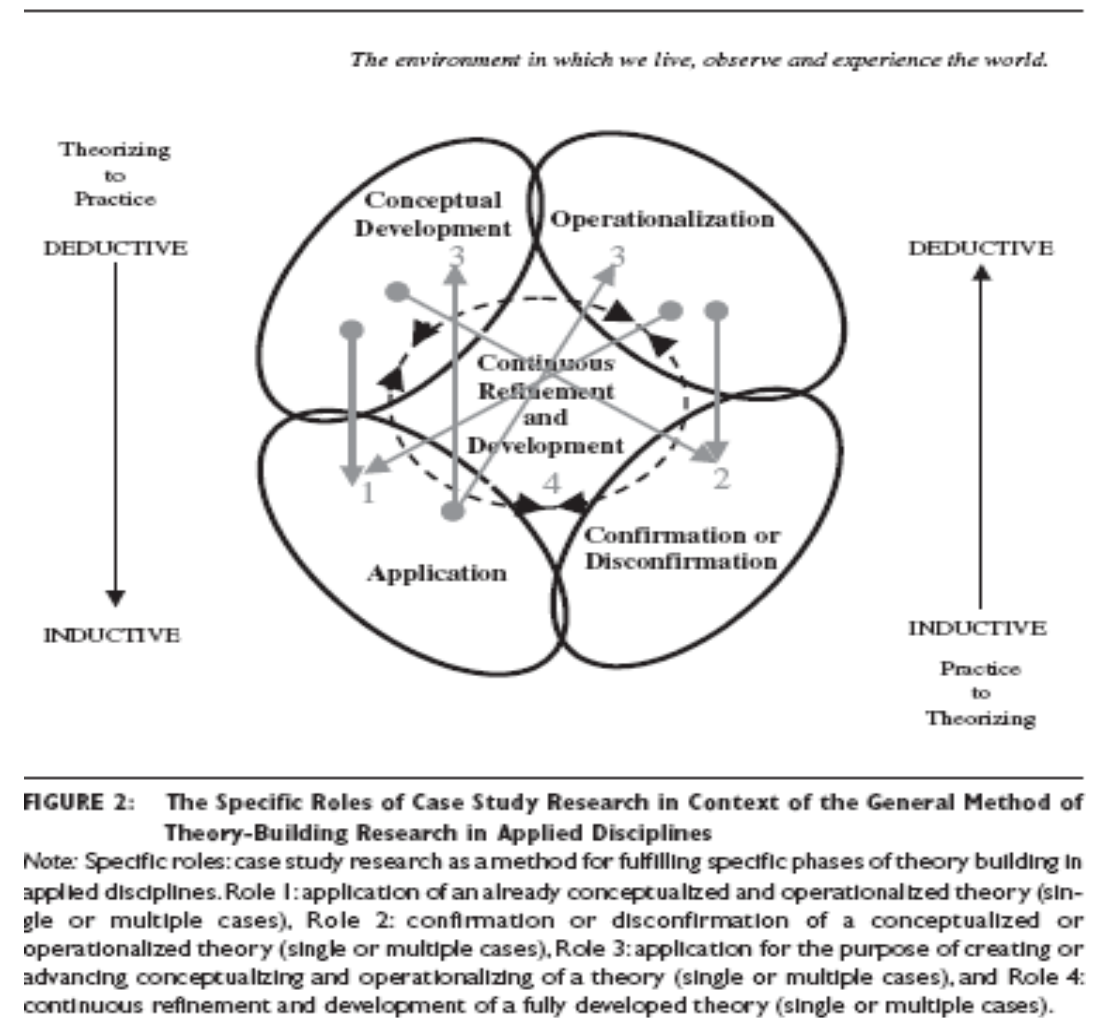

Figure 10: Specific roles of case study research in the context of general method of theory building.

(Dooley, 2002, p. 352)

In the context of case study research, Dooley (2002) suggests two key roles that case study research plays in the process of theory building because in itself, case study research is not a theory-building method. The two main roles are:

- a "specific role" in following the steps in a general method of theory building in applied disciplines (Lynham, 2002); this role requires fulfilling four sub-roles as outlined by Lynham's general method of theory building (see Figure 10 above).

- an "overarching role" bringing together multi-paradigm research and methods involved in different phases of a general method of theory building to shape what Eisenhardt (1989) refers to as a creative theory. 
To summarise, in the context of the above theory-building approach and the key stages in case study research, the design and conduct stage of case study research corresponds to the first two phases (conceptual development and operationalisation) of theory building. The confirmation or disconfirmation phase corresponds partially to the conduct stage of case study research, and more closely to the analysis and reporting phase of theory building. The application phase corresponds more closely to the reporting stage of case study research. The continuous refinement and development phase of theory building corresponds most closely to the last two stages of case study research.

With the rationale for case study research and the role it plays in theory-building outlined, the following section provides details of its description and application. Instead of detailing individual contributors' key methodological steps, an integrated approach is adopted here that considers the overlaps and commonalities.

\section{Description and Application of the Methodology}

The four distinct stages of design, conduct, analysis, and reporting of cases are based on the work of Yin (2003), Eisenhardt (1989) and Miles and Huberman (1994) on case study research. These stages are explained in detail in the following sub-sections:

\section{Design of the Case Study}

Yin (2003) and Eisenhardt (1989) proposed the following points as critical in designing case studies:

- the study's research questions

- the study's propositions and any a priori specification of constructs

- definition of the unit of analysis, selection, and the number of cases

- development of case study protocol

- the logic linking the data to the study's propositions and criteria for interpreting the study's findings

\section{Research Questions}

The above points are explained as follows. As pointed out in Chapter 3, the study's main research questions are: 


\section{Q1. What are the main factors that influence a firm's decision to invest in training in IT sector, within the Indian context?}

\section{Q2. Do an organisation's clients/customers have an impact on investment in training? If so, why, and how do they influence it?}

\section{Q3. How do various factors - internal and external to the firm - interact with one another in the provision of training in India's IT sector?}

\section{Q4. Why do some firms invest more than others, even if they are in the same industry sector?}

Study's Propositions and A Priori Specification of Constructs

Based on the review of theoretical perspectives covered in Chapter 3, the study will cover the main factors identified in the literature as significant in training decision making. It will also consider factors not previously explored, for example, the influence of an organisation's clients or customers on the provision of training. The conceptual development and operationalisation phase of theory building was covered in Chapter 3 . Study of the organisations in their real-life context will enable the development of a better understanding of the complex nature of interactions between various factors, both how and why they interact with each other in shaping the nature and extent of training. Such knowledge will deepen our understanding of why there is variation in the nature and extent of training provided between, and even within, organisations.

Although the main propositions of different theoretical perspectives outlined in Chapter 3 will be confirmed or disproved, the approach adopted here is an open and inclusive one, wherein, in addition to the key factors noted in Chapter 3, an examination of any new factors not previously identified will also be considered (Denzin \& Lincoln, 2005). Case study researchers emphasise that having a priori constructs and theoretical underpinning helps in shaping the focus of a study and enhancing the construct validity (Yin, 2003; Eisenhardt, 1989; Miles \& Huberman, 1994). However, the open nature of data collection and analysis will help in reducing the most common criticism: imposing a framework for interpreting the data. Further, participants' understanding of the key factors and their significance may allow for different understandings of the same phenomenon. 
Informed by the review of the literature and the contributing, useful, and novel theories (See Chapter 3), the following propositions are advanced:

Proposition 1: Firms will invest more in firm-specific training than general skills training.

Proposition 2: Firms will invest in general skills training due to asymmetrical market information about their new employees.

Proposition 3: The demand for highly skilled workers will decline with experience on a given technology.

Proposition 4: Where the focus of implementing new management practices is on increasing employee autonomy and discretion, firms are more likely to invest in general and technical training. Where the focus of such practices is on high employee control and low employee discretion, firms will invest in technical training.

Proposition 5: Firms focusing on a cost leadership strategy will invest less in training and quality management systems.

Proposition 6: Firms servicing slightly and highly differentiated service markets will invest more in training and quality management systems than firms servicing mass service markets.

Proposition 7: The strength of an organisation's quality management systems helps in shaping the nature and extent of training provision.

Proposition 8: In high-tech services environment, high levels of market and learning orientation will require higher levels of investment in technical and general skills training.

Case Definition, Unit of Analysis and Selection

Defining the case and the unit of analysis is an important step in the design of case study research. Stake (1995), for example, defines a case as an "integrated system" that is temporally and contextually bounded. Miles and Huberman (1994) view the case as the unit of analysis; they also admit that sub-cases can be embedded in the main case or unit 
of analysis. Following Yin's (2003) guidelines, a single case can have more than one unit of analysis. Depending on the focus of the research and problem at hand, the design may include multiple cases and holistic or embedded units of analysis. A clear definition of the unit of analysis helps in specifying the boundaries of a theory and its limitations.

Given the multi-case embedded design followed in this research, the case is defined here as understanding the factors that influence training investment in the IT sector's services firms in India. The case excludes IT hardware-manufacturing firms. As noted in Chapter 2, the IT sector comprises three broad divisions: IT hardware manufacturing, ITeS/BPO, and ITSS. Given that the focus is on services, only organisations from the latter two subsectors are selected in the sample. Within each case organisation selected, a minimum of two sub-business units or service lines are selected as units of analysis to understand how training decision making is influenced by a range of organisational and contextual factors.

A common criticism of single-site case study research is the lack of generalisation or transferability of findings from one case to another (Pare, 2004). In case studies, generalisation is not applied to the population using statistical sampling logic. Instead, generalisation is applied to a theory or a set of theories. Sampling multiple cases allows for external validity of the findings, but the extent to which one wants certainty is a question that the researcher has to decide (Yin, 2003). Yin (2003) suggests that cases should be chosen with a replication logic similar to that is followed in multiple experiments. Yin (2003) describes it in this way:

For example, upon uncovering a significant finding from a single experiment, the immediate research goal would be to replicate this finding by conducting a second, third, and even more experiments. Some of the replications might have attempted to duplicate the exact conditions of the original experiment. Other replications might have altered one or two experimental conditions considered relevant to the original finding, to see whether the finding could still be duplicated. Only with such replications would the original finding be considered robust and worthy of continued investigation or interpretation. (p. 47)

Following Yin, a multi-case site selection should aim at either a literal replication (one that predicts similar results) or a theoretical replication (one that predicts rival results but for understandable and predicted reasons). Given the two broad sub-sectors (ITeS/BPO and ITSS) and their business models (services environments and product environments) operating in India's IT sector, the present case study aims for theoretical replication across, and literal replication within, these two groupings. Yin (2003) suggests that a 
literal replication can be claimed if a phenomenon is found to exist in two or three cases. Similarly, four to six cases might be selected for two rival theoretical replications. The replications claimed can be informed by a theoretical framework, but need not necessarily be so. Such an approach allows for new theoretical categories to emerge from the data collected and analysed.

In the present study, a maximum variation purposive sampling approach is followed in the selection of cases (Eisenhardt, 1989; Miles \& Huberman, 1994; Yin, 2003). This approach allows for extending any existing theoretical categories and claiming replications in diverse conditions. Such a sampling strategy allows for robust theorybuilding. Finally, such a sampling approach is important considering the firm level differences in performance and the uneven profile of firms that exist in India's IT sector (Ethiraj et al., 2005; Heeks, 1998).

Case selection utilised the following criteria to observe the phenomenon in a diverse set of circumstances. The criteria were developed using extant literature on enterprise training (Boxall, 2003; Smith \& Dowling, 2001; Ashton \& Sung, 2005) and literature that considers the unique nature of India's IT industry (Banerjee, 2004; 2006; Heeks, 1998; Ethiraj et al., 2005):

- Enterprise size: (expressed in terms of employee headcount) small (less than 150), medium (151-1000), large (1001-3000) and very large organisations (more than 3000)

- Ownership: MNC, Indian, and MNC joint venture

- Business model: wholly owned captive centre of a MNC, third-party service provider, a mix or a variant of the above two categories

- Nature of services: product environment or project environment

- Nature of product-market strategy: mass production (with low value and complexity service), slightly differentiated services (with medium to high billing rates and service complexity), and highly differentiated and specialised services (with high value and service complexity).

It was ensured that in selecting the above cases, the chosen criteria were satisfied to allow for an understanding of the above phenomenon in diverse cases, within a single industry sector. The advantage of selecting cases from one industry over selecting multiple 
industry sectors reduces extraneous deviation. The findings can thus be much more focused and in the context of a sector's unique environment. There is likely to be minimum variation on account of internal and external labour market dynamics, because the sample does not cover diverse industry sectors, for example, pharmaceutical and steel manufacturing. Likewise, the global nature of India's IT sector does not have the regulatory trade barriers that are evident in some other service sectors, such as telecommunications and power. The above approach does not suggest that there are no within-sector and firm-level differences in performance and strategy; instead, it allows for a logical basis for comparison. Thus, selection of organisations from a single industry sector helps in better cross-case comparisons and helps to improve construct and external validity.

From an initial list of 19 organisations, using NASSCOM's directory of ITeS/BPO and ITSS organisations in India, 12 organisations agreed to participate, but a final list of 10 organisations was selected. This selection was based on the extent to which organisations were willing to grant access for the study and the extent to which the selection criteria were satisfied. Before seeking consent from respective organisations, the University's Human Ethics Committee's approval was sought for the entire project. An information sheet about the project (Appendix 1) and consent form (Appendix 2) were sent to the targeted organisations. Their consent was obtained in writing before data collection at various sites in India was begun. Figure 11 provides a brief description and illustration of the theoretical criteria for the ten cases selected.

\section{Development of case study protocol}

Yin (2003) emphasises the need to develop a case study protocol and database as it helps in the reliability of the study and allows others to follow the documented procedures, decisions and rules. The protocol contains more than just the interview schedule. It contains an overview of the project, field procedures (key contacts, access to site details, information sources), the interview schedules or guides, and a guide for developing the case study report. Details of the case study protocol can be found in Appendix 3. In Appendix 3, the project overview has been reduced to avoid repetition of information. Full details of the field procedures and interview instruments are provided in the protocol. A separate database was created for each case organisation (to enable focused within-case analysis). A database was also created for cross-case analysis. The database included field notes, transcripts, coding manual, protocol, organisational documents, news clippings, 
operational data, quality management performance data, and tables, matrices, displays and figures for analysis.

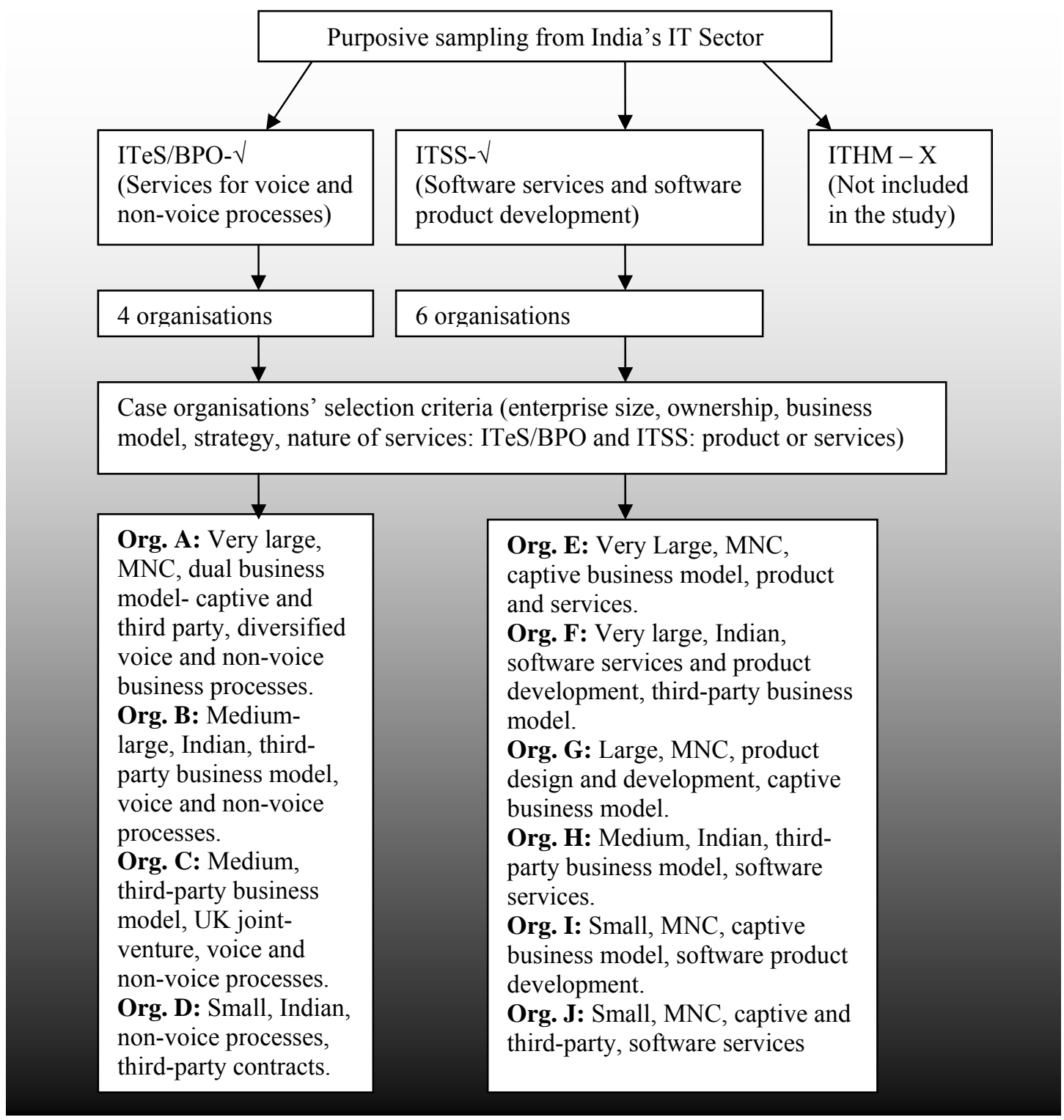

Figure 11. List and criteria for selection of multiple cases.

Case study protocol and semi-structured interview questions. Based on the review of literature, theoretical propositions were developed and linked to the study's research questions (see Table 4b in Chapter 3). To fully explore the propositions and answer the study's questions, semi-structured questions were developed in the case study protocol (see Appendix-3). The questions were developed primarily, from a priori concepts and, from the gaps identified in the literature (see Chapter 3). Following careful content analysis and review of literature, the main elements of each concept/theory was noted and semi-structured questions were developed for each sub-construct or aspects that were critical in answering the study's research questions and propositions. 
For example, following Kohli et al.(1993) and Narver and Slater's (1995) conceptualisations, the concept of market orientation, which has the following key behavioural elements: information sensing and dissemination from an organisation's current and potential clients and competitors and, inter-functional coordination for framing an appropriate organisational action or response, were used in eliciting information about this key strategic marketing concept. The protocol developed semistructured questions for understanding the extent to which a firm's market orientation is developed and how it impacts training provision at various stages of service delivery (see Appendix-3, pages 344-345).

Similarly, based on the TQM content analysis from the extant literature (Dean \& Snell, 1991; Prajogo \& McDermott, 2006; Reed et al., 1996, 2001; Sitkin et al., 1994), among other questions, the semi-structured questions probed the impact of key quality management themes such as customer satisfaction, continuous improvement, systemic view of an organisation, team working, information sharing and communication on training and other organisational processes (see Appendix-3, pages 342 and 345). The extent to which these themes were probed also depended on nature of the respondents and their position in the organisation.

\section{Logic linking data to propositions and interpreting findings}

Following Yin (2003), pattern-matching and explanation building analytic strategies were applied to different data from within- and cross-case analysis to the study's theoretical propositions. Further details are provided in the upcoming section on Analysis of Case Studies.

\section{Conduct of Case Study}

The conduct of the case study requires the researcher to decide how to collect the data and from which sources. Yin (2003) outlines six main sources of evidence: documentation, archival records, interviews, direct observations, participant observations and physical artefacts. Data collection for this research relied primarily on semi-structured interviews. Where access was allowed, documents in the form of organisational policies, training plans, performance appraisal systems, recruitment and selection data, and other publicly available information (news clippings and official websites) were also collected. Non- 
participant observation was also undertaken, but to a limited extent. Such an approach enhance the construct validity.

Even before the actual field work started, secondary data about each organisation was collected through the organisations' websites and published news clippings in the local dailies, business, and technology magazines. Examples of such resources include: The Economic Times, Times of India, India Today, Business Today, Dataquest, and NASSCOM publications. Some prior background information helped in understanding the key focus areas of each business and in developing a preliminary, albeit a "media" profile of each organisation. The "public relations" or "media briefs" nature of this information was kept in mind in order to avoid forming any firm and "glossy" views about the nature of each organisation and to remain objective for the data collection and analysis that was to follow.

Non-participant observation cannot be avoided while researching in real-life environments. Observation offers an opportunity to observe daily routines of employees in their real life. It helps in developing a contextual understanding of employees' work and, the organisational culture, and gives some sense of what's going on. Key points from observation were summarised at the end of each day's field work. The nature and extent of organisational documents and observation data varied owing to the varying levels of access provided by each organisation.

The use of an interview schedule helped in cross-checking the data from different informants. The semi-structured nature of the questions allowed for flexibility in data collection, especially if any new information emerged in the course of the interview that needed further exploration. While the interview schedule ensured consistency across multiple informants and case sites, a semi-structured approach enabled the collection of organisation-specific contextual information. Such an approach is useful as every organisation differs in its strategy and approach to work organisation.

Although the interview schedule provides structure and consistency for collecting data, a common consideration is to decide who to interview and how many interviews to conduct. Extant research in the area of enterprise training mostly followed a crosssectional design eliciting information from HR and/or industrial relations managers, CEOs, operational or functional heads, union delegates, employees, and their immediate 
supervisors (Smith \& Dowling, 2001; Smith \& Hayton, 1999; Ashton \& Sung, 2006; Dawe, 2003). These studies used a sampling strategy that allows for triangulation of data to obtain diversity of views about the same phenomenon. In this study, a purposive maximum variation sampling strategy was applied to obtain data from the following informants:

- CEOs or country heads

- HR managers

- training managers

- project or process managers

- project or process employees

- business development managers

- quality managers.

To help obtain consent for participation, details of data sources (informants, documents, observation) were provided via email communication to case study organisations. During the course of seeking consent, it became apparent that some of the information sought could be provided by designated business development and quality managers. Therefore, they were specifically added as informants to this study to get an understanding of how information is collected and disseminated from client sites to the service providers and to ascertain the specific role quality management plays at the different levels of its operation.

All organisations were visited during the December 2005 and January 2006 period. Depending on the level of access provided, the number and nature of informants varied in each organisation. The precise number of informants interviewed are provided in the case study protocol (Appendix 3). In some organisations, one individual performed more than one role. For example, in some cases, the CEO or country head was also the business development manager. Likewise, some project/process managers were also responsible for the implementation of quality management approaches in the organisation. This was typically the case in small and medium organisations or start-ups, where resources were limited. 
Given the multi-case, embedded case study design, the focus of interview questions varied slightly at organisational and project/process levels. Broadly, the interviews' focus can be classified as follows:

\section{Organisational level}

- background, ownership and history

- strategy

- work organisation and workforce composition

- workplace change

- role of quality management approaches

- HR and training strategy

- market orientation and learning orientation

- nature and extent of training provision

\section{Project or process level}

- specific work organisation

- $\quad$ specific HRM practices

- process or project work flow

- temporal dimension of training in a process/project life cycle

- $\quad$ specific training and development practices

- role of quality in training and service delivery

- process or project transition from client to service provider.

In only three of the ten case study organisations were some informants uncomfortable with recording the interviews. In such cases, the researcher made written notes of each of the interview questions. On average, the interviews ranged from 60 to 120 minutes. All the interviews were conducted in English and transcribed verbatim (where necessary, with grammatical corrections). While the researcher did most of the transcription, some transcription help was used. For reasons that follow, this help resulted in almost as much work for the researcher. Firstly, the transcriptionist in New Zealand could not cope with the strong and multiple, regional mother-tongue accents of the interviewees from different parts of India. Secondly, Hinglish (a combination of English and Hindi words), although sparingly used by some interviewees (where they needed to emphasise a point), created problems for the transcriptionist. Use of Hinglish is very popular in India because the focus is on being understood correctly rather than on using incorrect English. 
Consequently, where help was provided, all the transcripts were carefully checked by the researcher for verbatim accuracy, and where Hinglish expressions were used in the interviews, they were translated back to English.

Full confidentiality regarding the organisation and the informants' names was assured when consent was sought and was demonstrated in the case study drafts and the withincase analysis. Each participating case study organisation was sent a copy of the case study's draft report for validation and accuracy. Each was asked to comment on the technical accuracy of the data collected keeping in mind the state of affairs when the researcher visited the organisation. Each was asked to comment on the relationships between different factors (that influence provision of training) covered in the case study report. In some cases, the organisation requested that factual errors be corrected before release of the report.

Feedback and validation from the case organisation was often a time-consuming process. Organisations took a lot of time before releasing the reports as some adopted the approach of verification by all the informants in a study, some of whom had already left the organisation. In some cases, the main contact person for the case study had left the organisation, creating further delays, especially when the contact had left the country. Here, the contact details of other informants were used to find someone equivalent in the organisation to look at the case study drafts for accuracy, feedback and release. In two cases (Organisation F \& J), all the informants had left the organisation. In such cases, the researcher contacted the registered office or emailed the report to their equivalent or business contacts and requested feedback and release. The key contacts for a further two organisations (Organisation B and H) did not respond. The respondents' feedback form is provided as Appendix 4. The case drafts sent to participating organisations and the ones included here in the within-case analysis are different because the target audience is different and the focus in the first draft was to get as much description, explanation and analysis as possible approved and validated by the organisation for release. In some cases, factual errors were pointed out and amendments were made to case study reports. In other cases, where further clarification was sought from the organisation, teleconferences were arranged. The nature and extent of interaction and clarification sought varied among the organisations. The outcome was a shared understanding of the phenomenon. 


\section{Analysis of Case Studies}

The amount of data that is generated in multi-case, embedded case-study research, using triangulation of data sources can be daunting. The aim is to develop a more coherent understanding of the phenomenon under study (Kaplan \& Maxwell, 1994). Based on the work of Yin (2003), Eisenhardt (1989) and Miles and Huberman (1994), broadly, data analysis undergoes three distinct phases: early steps in data analysis, within-case analysis, and cross-case analysis. In each of the three phases of analysis, Miles and Huberman suggest adopting a pre-structured case study format. The process of analysis is cyclical and iterative, as shown in Figure 12.

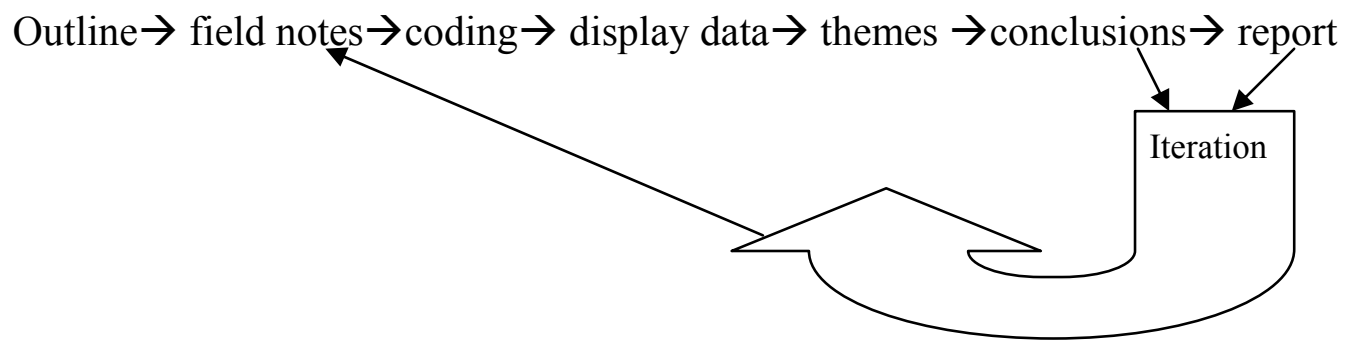

Figure 12. Typical data analysis sequence.

(Adapted from Miles \& Huberman, 1994, p. 85)

At the start of analysis, the researcher heard all the audio tapes at the end of each day of field work and made field notes based on the recordings. Additional field notes were added based on the observations from the day's proceedings. All transcripts were read at least twice to allow for reflection on the field notes and observation summaries. The interview transcripts were coded based on a priori concepts. However, given the open and flexible nature of the coding scheme adopted in this study, additional codes emerging from the data were inductively added for further exploration and analysis. Such an approach allows for rival explanations to be accommodated in the analysis and helps in refining or extending a priori concepts or identifying new codes.

Use of pattern codes helped in identification of themes and relationships emerging from various a priori and new theoretical categories. Such codes were developed using reflective notes and memos. Each theme and pattern was based on multiple observations and was accompanied by an analytic text in all the case reports. In this study, field notes, reflective remarks, organisational documents, and narratives were used in the development of a case study database. 
The typical structure of a case study database is as follows:

- field notes, interview transcripts and observations

- organisational documents and their preliminary analysis

- other documents collected or summarised about the organisation, for example information from their website and media clippings

- narratives and reflective comments written during analysis

- different types of coded data: descriptive, relationship and pattern codes.

\section{Within-Case Analysis}

Miles and Huberman point to two types of within-case analysis: describing and exploring, and explaining and predicting. Common analytic strategies noted in withincase analysis are: use of a dominant mode of analysis (Yin, 2003), use of visual displays (Miles \& Huberman, 1994), and review of case reports by participating organisations (Miles \& Huberman, 1994; Patton, 2002; Yin, 2003). Each of these strategies is explained below.

Dominant mode of analysis. In case study research, Yin (2003) suggests the use of a general analytic strategy using three possible analytic strategies:

- pattern matching

- explanation building

- time-series analysis.

Time-series analysis was not used in this study. In pattern-matching, an empirical pattern is compared with the predictions of a priori concepts. The focus is on finding patterns of factors that appear to influence training provision in IT organisations in India. Then the focus shifts to identifying any additional or new factors that are typical of India's contextual environment and are not found in the extant literature as factors driving training provision. Before any factor was included in the case reports and analysis, the researcher looked for more than two sources of evidence by checking the transcripts, observation notes and, where possible, organisational records.

Explanation building, while similar to the pattern-matching strategy, focuses on developing explanations of how and why certain factors influence provision of training. 
This analytic strategy considers the interaction of various factors. Similar to pattern matching, explanation building also requires a logical chain of evidence to be developed for each explanation.

Use of visual displays. During within-case analysis of each case site, preliminary data reduction and the use of data displays force the researcher to condense and display the different categories of data present in each case. Displays are useful in analysing and exploring similarities and differences between the categories of data analysed. Data display preceded the verification and conclusion stage of data analysis. In within-case and cross-case analyses, different types of matrices and displays (for example, sequential displays, conceptually clustered matrices, and explanatory maps) were developed to analyse the presence of certain relationships or a temporal sequence.

Review of case reports by informants. As noted earlier, all case study draft reports were sent to the participating organisations to strengthen the credibility of the interpretations and validity of findings. Review of case drafts by participating organisations helps in dealing with the embedded and holistic aspects of the case analysis.

\section{Cross-Case Analysis}

Firstly, cross-case analysis identifies themes and patterns found across multiple sites. Secondly, it offers a deeper understanding of the phenomenon across a range of cases. Finally, it helps in identifying rival explanations to develop a robust conceptual framework. Cross-case analysis involves study of within- and inter-group similarities and differences. Again, the use of visual displays, explanatory networks, and the development of matrices forces the researcher to look for similarities and differences, and, where differences exist, to look for explanations of why they exist, thereby extending the theoretical categories.

\section{Writing Up of Case Study Reports}

Yin (2003) suggests keeping in mind the target audience when reporting on case studies. In the development of case study reports, the researcher was aware of two audiences: the participating case study organisation and an academic audience. The audiences require somewhat different formats, content and presentation styles. When the case draft was submitted to the participating organisation, it was a detailed document containing three 
parts: a background paper (containing the study's questions and an overview of theoretical perspectives), a detailed case study draft report, and an instruction sheet for evaluating the report and for providing feedback. The case reports presented in the thesis are condensed versions keeping in mind the University's word limit requirements.

\section{Conclusion}

This chapter provided the methodological basis for proceeding with a qualitative case study design. It demonstrated the study's methodological appropriateness and congruence. The research protocol and database allows for the study's reliability. The use of a coding scheme, displays, and matrices to sort and analyse data, and a standard case reporting format allows for comparison in cross-case analysis and demonstrates the methodological rigour followed. The findings and propositions from this theory-building effort can be further tested using well-established survey methodologies and following Dubin's (1978) hypothetico-deductive theory-building approaches. The following chapter provides within-case analyses of ten case study organisations. 


\section{CHAPTER 5}

\section{WITHIN CASE ANALYSIS}

This chapter provides detailed within-case analysis of the ten case study organisations selected in this study. For ease of analysis, comparison, and reference each case is alphabetically identified according to its brief description in Figure 11 of Chapter 4 and is numbered as ten sub-chapters 5.1, 5.2, to 5.10. As such, the within-case analysis is organised according to the following headings:

- About the organisation

- Organisation structure and processes

- Business processes, strategy, and work organisation

- Nature and extent of training

- Analysis of factors influencing provision of training

- Specific impact of factors on nature and extent of training

- Discussion

- Conclusion

Using theoretical perspectives to guide the data collection and following the case study protocol (see Appendix 3), the emerging themes were analysed and coded (see Table 5). Additional coding categories were created for any new factors that emerged during the data collection and analysis process (See Table 5). Some factors (unionisation and impact of competition) that have been identified in the literature (and included in the protocol) as having an impact on training provision were dropped from the final analysis for reasons that follow. Unionisation was non-existent in the ten case organisations studied. The impact of competition on training was not identified by respondents as a critical consideration owing to high industry growth rates. Similarly, as there was limited financial and time support for employees to pursue higher educational qualifications from external educational institutions, learning support was not included in the final analysis. Owing to definitional problems encountered during the research process, measure of training volume (extent) was re-conceptualised to focus on the "time" allowed for training (formal and informal). Further, large firms offered opportunities for higher education through their internal "corporate university" programmes. Such investment was captured in terms of "work time allowed" for study. Similarly, where training provided was informal, respondents' estimate of time was considered to compute the extent of training. 
Table 5 .

\section{Key categories}

\begin{tabular}{|c|c|c|}
\hline \multirow[b]{2}{*}{ Category codes } & \multicolumn{2}{|r|}{ Origin } \\
\hline & $\begin{array}{l}\text { A priori (Theoretical } \\
\text { underpinnings) }\end{array}$ & $\begin{array}{l}\text { New and/or extended theoretical categories arrived } \\
\text { through iteration (with comments what is new and how?). }\end{array}$ \\
\hline $\begin{array}{l}\text { Workplace change (WC) } \\
\text { Structural } \\
\text { Ownership } \\
\text { Technological }\end{array}$ & $\begin{array}{l}\sqrt{ } \text { (Various Neo-HCT } \\
\text { and HPWP studies) }\end{array}$ & $\begin{array}{l}\sqrt{ } \text { (New types of change: vertical and domain based. Other } \\
\text { changes include Platformisation - a technological change) }\end{array}$ \\
\hline Competitive strategy & $\sqrt{ }($ Boxall, 2003) & Coexistence of different competitive strategies in a firm \\
\hline $\begin{array}{l}\text { Market orientation (MO) } \\
\text { information sensing } \\
\text { information dissemination } \\
\text { organisational response }\end{array}$ & $\begin{array}{l}\sqrt{ } \text { (Kohli et al., 1993; } \\
\text { Sinkula et al., 1997) }\end{array}$ & $\begin{array}{l}\sqrt{ } \text { (MO abilities can be geographically distributed. Sensing } \\
\text { versus dissemination and response or, other combinations) }\end{array}$ \\
\hline $\begin{array}{l}\text { Learning orientation (LO) } \\
\text { commitment to sharing } \\
\text { open-mindedness } \\
\text { shared organisational vision }\end{array}$ & $\sqrt{(\text { Sinkula et al., 1997) }}$ & $\sqrt{ }$ (New relationship with client specifications) \\
\hline Client specifications (CS) & & $\sqrt{(\text { New factor: not previously identified in the literature) }}$ \\
\hline $\begin{array}{l}\text { Quality management } \\
\text { systems (QMS) } \\
\text { sharing information } \\
\text { continuous improvement } \\
\text { teamwork }\end{array}$ & $\sqrt{(\text { Reed et al., 2000) }}$ & $\begin{array}{l}\sqrt{(\text { New relationships with other factors; LO, MO and }} \\
\text { strategic HRM practices })\end{array}$ \\
\hline Employee turnover (ET) & $\sqrt{ }$ & $\sqrt{ }$ (Positive, instead of a usual negative impact on training) \\
\hline Enterprise size (Size) & $\begin{array}{l}\sqrt{\text { (Hayton et al., 1996; }} \\
\text { Smith \& Hayton, } \\
\text { 1999; Ridoutt et al., } \\
\text { 2002) }\end{array}$ & $\begin{array}{l}\text { (It is more service/business complexity than enterprise } \\
\text { size that impacts nature and extent of training) }\end{array}$ \\
\hline Process/project complexity (COMPX) & & $\begin{array}{l}\sqrt{ } \text { (New factor: business and service complexity versus } \\
\text { high or low service specifications) }\end{array}$ \\
\hline Ownership (OWN) & $\sqrt{(\text { Hayton et al., 1996) }}$ & \\
\hline Temporal dimension (TD) & $\begin{array}{l}\sqrt{ } \quad(\text { Bartel \& } \\
\text { Lichtenberg, 1987) }\end{array}$ & 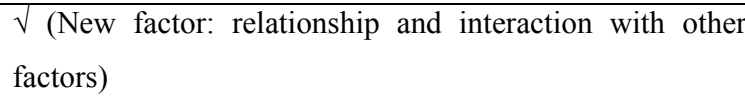 \\
\hline Geographical dimension (GD) & & $\sqrt{ }$ (New factor) \\
\hline $\begin{array}{l}\text { Strategic HRM (SHRM) } \\
\text { rewards \& performance management } \\
\text { systems } \\
\text { career planning } \\
\text { recruitment skills level \& operational } \\
\text { roles } \\
\text { casualisation }\end{array}$ & $\begin{array}{l}\sqrt{ } \text { (Various SHRM } \\
\text { models in Chapter 3) }\end{array}$ & $\begin{array}{l}\sqrt{ } \text { Nexus of HRM and training with operations } \\
\text { management }\end{array}$ \\
\hline Extent of training (VOL) & $\sqrt{(\text { Hayton et al., 1996) }}$ & Description from extant literature not fully applicable \\
\hline $\begin{array}{cl}\text { Nature of training (DIV) } \\
\text { - } \quad \text { formalisation } \\
\text { - } \quad \text { externalisation } \\
\text { - } \quad \text { type } \\
\text { - } \quad \text { transferability }\end{array}$ & $\begin{array}{l}\sqrt{(\text { Hayton et al., 1996; }} \\
\text { Smith \& Hayton, } \\
\text { 1999; Ridoutt et al., } \\
\text { 2002) }\end{array}$ & $\begin{array}{l}\text { Some descriptors from extant literature not fully } \\
\text { applicable }\end{array}$ \\
\hline
\end{tabular}

The following ten sub-chapters provide description and within-case analysis of the ten organisations. ${ }^{1}$

\footnotetext{
${ }^{1}$ Note: Findings from organisations A-D (ITeS/BPO firms) have been published in Malik (2009) and presented at the 2008 Academy of Management Meeting (Malik \& Nilakant, 2008).
} 


\title{
CHAPTER 5.1
}

\section{ORGANISATION A}

\begin{abstract}
About Organisation A
Organisation $\mathrm{A}$ is a business processes and technology outsourcing services provider to a number of industries around the globe. It has witnessed organic growth in its products and the industry sectors to which it caters. Recently, it has expanded its operations from India to seven other countries to keep pace with its skills needs and to improve its service proposition to customers (see Figure 13). Currently it has 24 process delivery centres world-wide and a pool of engineers who can converse in 28 different languages to deliver the services it offers.
\end{abstract}

Organisation A initially started operations as a wholly owned offshore BPO for a large and diversified US-based multinational organisation, but it was recently acquired by two large players in the global BPO market. Drawing on its heritage of lean production, Six Sigma (SS), and other quality methods, Organisation A follows quality management systems (QMS) to exact process requirements from its clients and to deliver cost and productivity gains to its customers (Organisation A Document, 2005). Its staff strength grew from around 350 people in 1997 to over 26,000 globally at the time of the visit, with over 20,000 in India in 2006. This rapid increase in employee base is also mirrored in its revenues, products, and its portfolio of services.

The organisation's services include sales and marketing analytics, supply chain and aftermarket services, financial services, core operations and collections, finance and accounting, information technology services, and enterprise application services and program management. Within these services, it has a diverse range of products, which it offers to range of industry sectors, such as banking and finance, insurance, retail, manufacturing, transportation, automotive, pharmaceuticals, media and entertainment, and professional services.

Given the research design and the time and resource constraints, it was not possible to cover all its business lines. Therefore, the researcher selected its Insurance Services, Finance \& Accounts (F\&A), and Content Solutions groups and met with their respective $\mathrm{HR}$ and process training leaders and other respondents, namely, AVP-HR Shared 
Services, a training black belt (a Six Sigma (SS) certification), and VP-Quality and Six Sigma (SS). The Insurance service line provides business processes outsourcing for insurance products, such as life, medical, home and content, industrial, and commercial risks insurances. The Content Solutions service line delivers design and development solutions for training projects for the organisation as well as its clients. Among other services, F\&A service line's accounts receivables and payables, and reconciliation were studied.

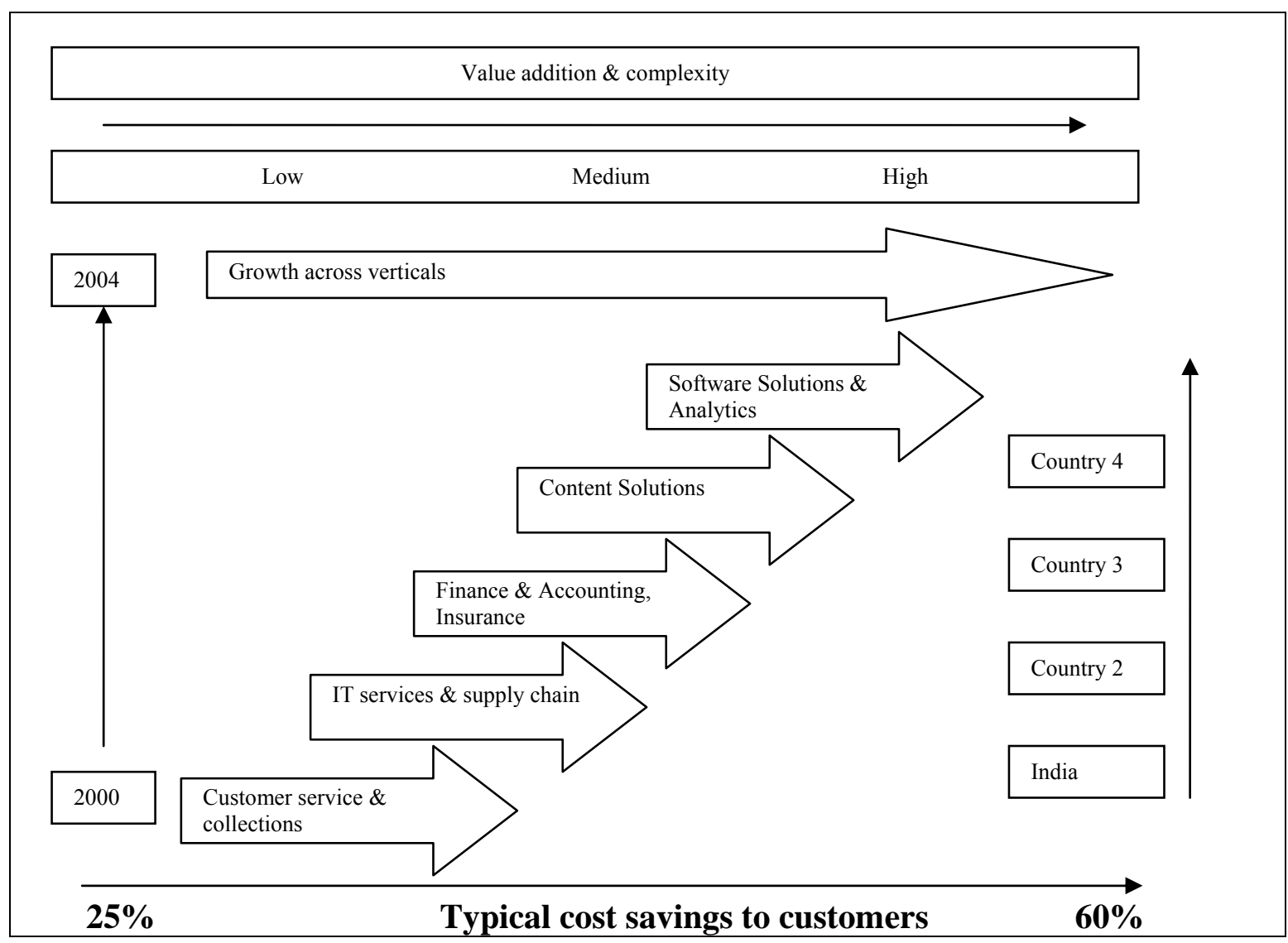

Figure 13. Business growth across verticals.

Adapted from Organisation A's documents available through the public domain

\section{Organisation Structure and Processes}

The organisation is structured around domains (service areas) and verticals (industry sectors). There are different groups servicing a range of domains. The organisation has five broad levels of hierarchy, wherein five is the entry level band for movement within and between service groups. Based on the figures and information supplied by the organisation, a typical Taylorist or Fordist work structure is prevalent, with the majority of the employees (97\%) in Bands 4 and 5. A very small executive and managerial team heads these service groups. 


\section{Business Processes, Strategy, and Work Organisation}

Before it is explained how business processes and work is organised, it is important to understand how the organisation secures business processes from its clients' site. Since its inception, Organisation A has transitioned over 1000 business processes for over 60 different businesses. Following its transition from a captive (wholly-owned) to a noncaptive (third party) service provider, it now receives business from several Fortune 500 customers.

Using a SS methodology, it follows a stepped approach to assess, migrate and deliver operations from offshore locations (Document A, 2005). It follows a structured workflow transition methodology and has elements of SS interwoven into it at all stages.

\footnotetext{
The Six-Sigma based work transition methodology to enable business process relocation is based on a toll-gate based, phased approach, and addresses both aspects of process migration - strategic and operational. Customer acceptance of significant milestones and periodic feedback enable effective and efficient work transition of business processes. (Organisation A Work Transition Document, 2005)
}

Its business development team is spread across the globe and identifies business opportunities based on its market intelligence and research. Organisation A's competitive strategy typifies a blend of mass service transaction services to and highly differentiated services (Boxall, 2003). It currently focuses on areas where it has strong service capabilities.

At an operational level, its work transition methodology ensures steps such as understanding clients' existing processes, solution identification, work transition set up and knowledge transfer, pilot operations, and service delivery. Each step is further broken down for project definition and scoping and involves developing high-level process flowcharts and collecting information based on business needs, and developing metrics around them, especially the metrics that are critical to quality (CTQ) and, hence, the performance of that process. These steps are followed by framing solutions, based on a cost-benefit and risk mitigation analysis. 
After solutions for a project are framed, the work is transitioned to an offshore location in India. This involves two stages: pre-transition and transition. In the pre-transition stage, the transitioning team develops a thorough understanding of the adapted work processes and the underlying technology and prepares detailed transition plans. Based on the strengths and process expertise of the solutioning team, the transitioning team collects detailed information necessary for knowledge transfer and, reviews the client's existing standard operating procedures for these processes, using its subject matter experts (SMEs) and SS-certified Black Belts (BBs).

During transitioning at the client site, all the information is sent simultaneously to the offshore location for working out detailed infrastructure and human resource plans. Infrastructure specifications such as number of employees and their job specifications are determined. Recruitment commences around the same time as pre-transitioning. The training team prepares for its training responses for the new process. The second stage of transition involves validating the process metrics by the clients, monitoring team performance, and finalising of statements of work with the client. The third step involves piloting the transitioned process, following which the process enters the delivery stage. During this stage, using SS tools, the focus is to implement defect reduction plans and to develop forums for auditing, analysing and sharing good practices within and across domains.

\section{Nature and Extent of Training}

In terms of the extent of training provided, Organisation A invests in more than 1.5 million people-hours every year (on average, 12 days per annum or approximately $5 \%$ of its payroll) through various training and development programmes. The nature of such training includes: Education@Work programmes, executive development, communication skills, process training, domain training, SS-certification and numerous other educational programmes offered by Organisation A University. In 2006, about 5,700 employees were enrolled in the Education@Work programme. The programme offers employees MBA courses from reputed institutes in India, as well as from other international institutions, for programmes such as PMP (Project Management Professional) and CPA (Certified Public Accountant) to offer education for people to learn and grow in certain areas. 
Training leaders representing every service group assist its central learning and development team. In addition to this structure, each group has their team of process trainers and training leaders. Sharing of training resources from various groups helps in bringing together common issues and "good practices" from their respective areas, to share them with others in the organisation. The above approach to learning suggests a strong presence of informal and formal learning. For example, in the insurance practice, there are over 15 trainers. The reason for such an elaborate training infrastructure is explained as follows:

\footnotetext{
The trainers will be at the process level, as the training manager would not be able to look at the details at every level. I will give you an example; say life insurance is one business line, and within life insurance, there are many areas. Now if I look at life insurance's overall business, there could be so many sub-processes, and one training manager will not be able to look after all the areas. So, what we do is we have a sub-process trainer between each of these business areas:
}

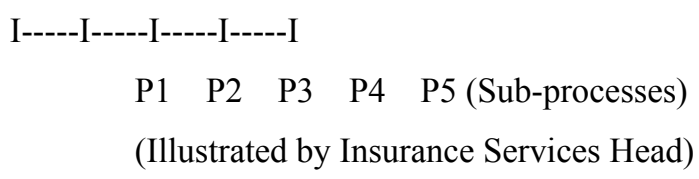

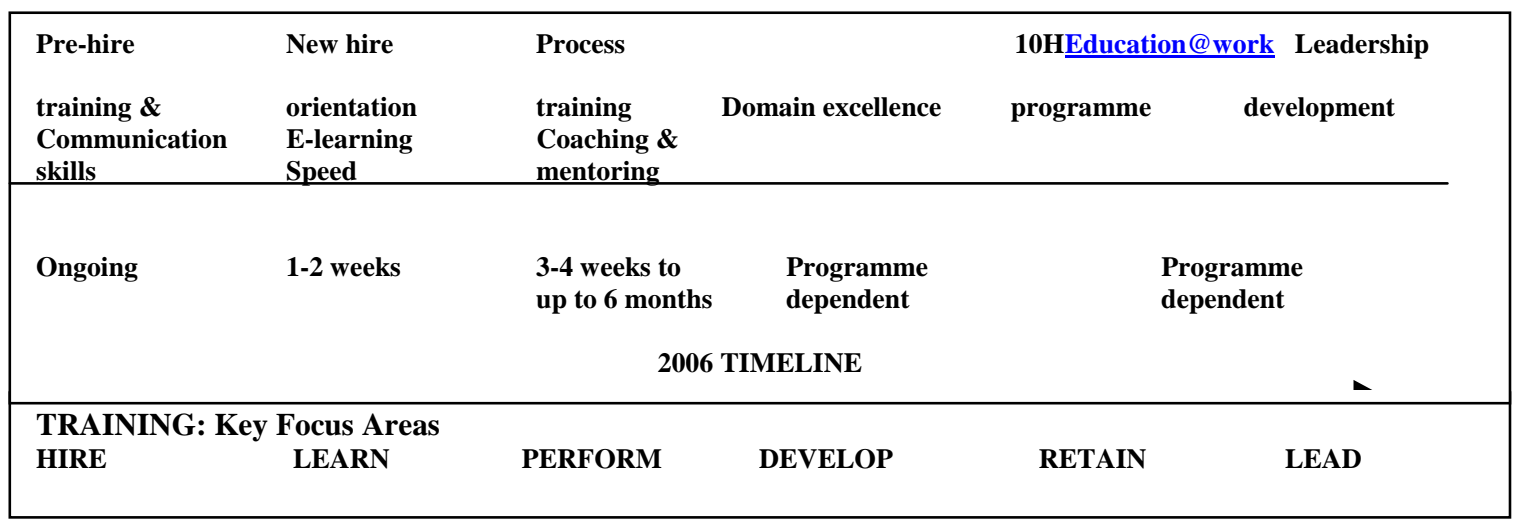

Figure 14. Temporal dimension of training: Changing emphases.

The organisation's emphasis on, and expectations from its training function change on an annual basis, and are part of its strategic reviews and planning process. This strategic approach to training was noted in organisational documents and observed during the case study visit. Figure 14 sets out these expectations. In the insurance service line, such training focuses on basic knowledge of insurance terms: premium, risk, agent, annuity, and claims. Trainees then undergo process-specific compliance and regulatory modules. Given the highly confidential nature of transactions, agents are required to learn and 
comply with specific laws from different countries or even state-level legislation that governs the nature of such transactions.

After the above modules are completed, employees then undergo process-specific training, which varies from process to process. As noted in the previous quote, the work is categorised into sub-processes. For example, in the Insurance service line, there are P1, P2, P3 and P4 processes. There are important distinctions to note here as the work organisation and classification is on the basis of process complexity. Simple processes, for example P1, would require a shorter training session compared with a highly complex process like $\mathrm{P} 4$, which requires significant training time and effort. Given the continuum of process complexity, training time varies from 3-4 weeks to up to 6 months. A typical P4 process in the insurance service line would involve training a medical doctor to read through the medical histories of insurance companies' clients to produce medical summaries and establish possible risks and linkages for insurance underwriting purposes. Such medical histories can sometimes be 800 pages in length and require specialist skills, professional judgement, and training. On the contrary, a typical insurance P1 process would involve training an employee to do data entry based on an image, as seen in a claim form. The employee enters certain types of data in pre-specified fields.

\section{Analysis of Factors Influencing the Nature and Extent of Training}

\section{Workplace Change}

In 2004, after Organisation A divested its majority share to two large multinational BPO firms, it introduced numerous changes to its structure, business practices and the nature and extent of training. Its old offshore process centre (OPC) business model, which essentially involved money transfer from the parent multinational to the Indian delivery centre, had to change. The resulting arrangement was a dual business model catering to captive and third-party clients.

Understanding the clients' needs in a third-party environment presented numerous challenges, such as the need to change the verbiage that is used in a captive environment to cater to the needs of a third-party environment, as one respondent explained. Senior managers needed to learn how to pitch out to clients in a non-Organisation A (captive) terminology. 
We have a typical acronym-centric language way of talking in a captive environment. It needs to be changed. Telling the clients the difference between level 1 and 6 of Six Sigma is important .... It's like how not to talk Organisation A, when you are not in Organisation A ... CTQ? We keep harping on it. Critical to quality ... should be performance standards. Training has a huge task to change the way we talk and operate. (Black Belt-Training)

For the short to medium term, the organisation had decided to operate in a limited number of domains and industry sectors. The strategic position is reviewed keeping in mind the third-party nature of business opportunities and its evolving sets of capabilities to tap into new areas. The focus presently is to develop the existing services further by tapping into the huge third-party BPO markets. For its captive business service lines, the technology platforms and processes across different client (parent organisation's) locations were very similar across its parent's business. However, in third-party contracts, differences exist on aspects of technology infrastructures, terminologies, and software applications. These differences have an impact on training. The culture at Organisation A supported people who embraced change through continuous training and skills development. In line with the above dual business model, Organisation A had to adapt its HRM practices to the changing strategic direction and to modify its recruitment, rewards and career progression practices to enable and support workplace change.

\section{Strategic HRM Approach, Workforce Composition, and Skills Level}

The presence of a strategic HRM approach was evident in its recruitment, career development paths and performance management systems. Hiring is linked to the level of skills needed for a process, which exists on a continuum of simple to complex processes. Depending on the service line and the processes complexity, recruitment specifications are determined. A high degree of process complexity related strongly to the need for highly skilled and qualified employees. As a significant number of its hiring was for voice processes, the organisation invested in, among other types of training, pre-hire voice training. This helped tremendously in the screening and the selection of right candidates. For complex non-voice processes, one of the respondents noted:

The software solutions group offers a lot of services in Oracle and related solutions so you hire those people who come with these specific skills. They come trained. Typically you would hire people who have these skills and definitely the training opportunities in these areas are very different from a transaction processing business. (Black Belt_-Training) 
Its HR services develop skills inventories for all its employees, with their proficiency levels placed against each role-specific competency, and has well-identified learning paths to allow individual development and career progression. The performance management and rewards system is geared to support the above, thereby reflecting a mix of technical and social work relations (Wilkinson, 1983, 2002). Its workforce composition does not include significant use of non-standard employment. The majority of employees are permanent full-time or part-time, with a very small number of contractors and casual employees in operational and technical roles. Moreover, the majority (97\%) of its employees are employed in operations, which consumes the bulk of the training-volume and diversity. Recruitment is based on the process complexity and focuses on strategic and cultural reasons. To contain employee costs, it hires relatively less skilled people for simpler roles. To address cultural needs, permanent roles have mass appeal and ensure a steady flow of people.

Strategically, investments in its training infrastructure are recouped by recruiting people with relatively lesser qualifications and using its existing training capability to train them for various processes. This method helps in managing the recruitment, performance, and service delivery costs. Each year, additional investments are made to further develop this capability and depending on the extent to which it can develop and deliver new skills, its ability to tap into a larger and relatively cheap and unexplored pool of less skilled workers increases. Such an approach enables sustained costs advantages over its competitors.

The above approach of providing upskilling opportunities to employees works well in a country like India. First, it is hard for students to seek admission into premier educational institutions owing to too much competition for too few seats. Second, the cost of quality higher education is rising. From the organisation's perspective, this approach helps in recruitment and retention, managing cost structures, and developing a steady supply of domain-specific skills and competencies.

\section{Quality Management Systems (QMS)}

Organisation A has a strong culture of Six Sigma (SS) and Lean Production embedded in its operations. Its extensive quality management systems and resources reinforce the SS 
and Lean management philosophy. The two philosophies complement each other. One of the respondents summarised:

\begin{abstract}
When you have a solution to a problem you use lean, where you don't have a solution to a problem you are going for Six Sigma. There are times when you don't have a solution to a problem, so there you dig deep and do a Six Sigma. You look at various causes and see what is addressable and what is not addressable. (Process Head-Insurance)
\end{abstract}

As noted earlier, its transitioning team has SS resources for developing metrics for clients' SLAs. The agreed metrics are then executed during the service delivery stage. Quality management systems are used to implement process improvement projects throughout the organisation through a team of about 200 SS-trained and certified Master Black Belts (MBB) and Black Belts (BB). The organisation has about 2000 Green Belts (GBs). Typically, these are SS-certified resources from operations. In addition, there are Quality Leaders (QL) deployed across different service lines. The training needs of dedicated SS resources are different from those of the rest of the organisation. In order to lead improvement projects, SS resources need to be familiar with the specific SS tools and methodologies. Apart from the normal SS training and certification, they are also required to undertake leadership training. SS training is mandatory for all Band 4 and above employees, whereas, Lean training is open to all but it is not mandatory.

Additionally, all managers have to be trained and certified as green belts within twelve months of their joining. They follow a structured 2-week internal training programme for SS, which draws on the internal expertise of SS resources and uses a formal internal testing and evaluation site for its assessment and certification. After completing the first week, trainees are expected to apply the concepts by working on SS projects in their service lines and then coming back to complete the second week of training.

BBs are full-time resources of the quality department; they do not have anyone reporting to them. BBs cannot bring about process improvements without having the buy-in of people on the shopfloor. Thus, the SS role is seen as a leadership role and influencing people is critical in an SS role. One of the respondents captured the importance and essence of SS philosophy at Organisation A:

The entire career of a black belt or master black belt is going to be disastrous if he/she is not able to influence people. You'll see that the best people in the organisation are six sigma people. ... They are part of developing and deploying the tools, which is why we have a 
very special six sigma DNA in the organisation. You will find that even at the lowest levels people are very well versed with the tools. I will be very surprised that if in a room of 30 people, even two people have not heard of fishbone. Yeah, it becomes part of our DNA. Everybody uses it. . . . It is so much there inside us that it becomes a part of our DNA. That's it, six sigma is a DNA now. (Vice-President-SS \& Quality)

This "Six Sigma DNA" is one of the key driving forces behind this metrics-driven organisation; its cultural heritage is another. Employees note that metrics excite them. Every process has internal and external metrics that form the basis of clients' SLAs. The presence of a SS philosophy appears to be driving formalisation and evaluation of training. A case in point is the content solutions service line, where metrics were developed for measuring "quality of customer delight". The presence of a strong "Six Sigma DNA" also influences its market and learning orientation. One of the respondents noted:

With the help of our Six Sigma team we are focusing on the quality of the solutions that we churn out in the end and the quality of the solutions architecture that we are recommending at the beginning. . . . Everything is metric driven; without that people won't survive. (Process Head-Content Solutions)

The quality team (SS resources) and process managers drive the collation of client feedback, development of metrics for SLAs and delivery, and constant improvisations. Each process has dashboards to capture, share, and monitor the metrics on that process. Dashboards were observable during the visit. Dashboards are useful for future benchmarking, since parameters and metrics change over a period of time, as clients request to work on a new metric. Developing internal standards of performance using SS methodologies enables transitioning and solutioning teams to propose what is do-able.

\section{Learning Orientation}

Organisation A demonstrates a very high commitment to learning. There are numerous platforms and forums for sharing good practices and learning from other parts in the organisation. These are organised at service, domain-specific and functional levels and enable the development of a shared vision. The frequency of such forums varies. Some meet weekly, others only quarterly. Examples of forums from functional areas include: general forums, new technology and market intelligence forums, project and domainspecific forums. In the learning group, learning leaders from various service lines meet 
monthly to discuss learning and development issues from their work areas. Functional and service-specific quick market intelligence (QMI) calls are made across various locations to share good practices and delivery issues.

The organisation's employees engage in double-loop learning, and frequently challenge their work and clients' assumptions. Challenging clients' assumptions happens before, during, and after process transition. First, when the solutioning team is designing solutions for a client, it proposes solutions that differ from the clients' existing standard operating procedures, to streamline processes at delivery end and to reduce errors. The ability to challenge clients' assumptions is based on established internal benchmarks and considers what is do-able using its SS expertise. The Process Head-Content Solutions noted: "In the last year, we would have given close to about 245 ideas on process improvements".

Finally, the organisation has put in place several mechanisms for embedding a shared vision across the organisation. Given its size, geographical dispersion, and the complexity of its processes, it is important to have an effective and efficient mechanism for communicating the vision and strategy. This is done in numerous ways, including the use of posters, electronic portals and message flashes through workstations and dashboards. Again, if the size of the communication is large and complex, it could translate into a formal training module. Alternatively, refresher courses or other kinds of informal learning occur. To develop a culture of learning, its key communications could be observed through posters, electronic message boards, and dashboards at various workspaces. Examples of messages include: process excellence, SME, customer delight, better performance, improved costs, passion, speed, decisiveness, integrity, best-in-class, and employer of choice.

\section{Market Orientation}

High market orientation is critical for the organisation as it transitions from its captive to a third-party mindset, because most of its contracts are long-term and future revenue is often dependent on additional and repeat business from existing clients. Organisation A has high levels of market orientation (MO). It is evident right from the time when the business development and solutioning teams are sensing information from its clients on various processes to be transitioned to India using its structured Six Sigma Workflow 
Methodology. Dissemination of information and framing of responses, in line with client specifications and service level agreements (SLAs) took place at various stages of the transitioning and delivery.

To understand clients' needs better, the organisation uses its reengineering solutions to help the customer streamline their processes even before initiating the transition using a structured Lean and SS workflow methodology. The benefit of this is simplification of the process even before transition, a benefit the client sees from the outset. In short, the organisation's solutioning strategy is to understand the individual customer's needs and see what solutions can be provided, keeping in mind the resources and capabilities it has for service delivery.

Because customer specifications are unique for each business processes and processes range from simple to complex, these different client specifications translate into different levels of training needs. Client specifications directly impact on training volume and diversity. The skills gap between client needs and existing capabilities is identified and communicated to the delivery teams, and training is provided through various training interventions at different stages of the outsourcing process: pre, during, and post go-live. For example, first, the solutioning and transitioning teams have to be trained in negotiation, quality management skills for developing solutions, and business and domain knowledge. During process transition, training plays a key role in knowledge transfer, as clients' standard operating procedures need to be integrated into the service provider's routines. Post go-live, process enhancement requests from clients require additional training.

Use of SS methodology ensures specific client and process metrics are agreed and integrated into the workflow and, where possible, post-transition, process improvements are made. Theoretically, there is always scope for process improvement and increasing the sigma levels, however, this need has to be felt and requested by the customer to have a business impact for both the client and service provider.

Organisation A's MO (information-sensing and dissemination) and LO (sharing and challenging of information) seem to be intertwined (Sinkula et al., 1997; Baker \& Sinkula, 1999 a, b). Further, the analysis reveals a link between its LO MO and quality management systems, particularly its Lean SS capability. QMS work as the catalyst 
between LO and MO, because they enable the development of a metrics-driven language for service definition, design and delivery.

The enabling effect of quality management systems on developing it's LO and MO seems to have in turn, a synergistic effect on the nature and extent of training provided. For example, when a client requests a process improvement or a change to the existing process, this usually involves in a change in the process at the delivery end by the development of new metrics using its QMS. Depending on the scale of change requested, these metrics get translated into formal or informal training. If the nature of change is small, an informal training session is organised, whereas formal training modules are developed for large changes to the existing processes. Further, the use of SS and Lean methodologies, as well as high levels of MO and LO have a profound impact on the what and how of the information to be sensed and, disseminated, and for the solutions to be framed.

The extent to which SS and Lean projects are undertaken is partly driven by clients' service level agreements (SLAs), and partly to bring about internal process efficiencies at the service providers' end. Internal process efficiencies result from its SS expertise, as it allows getting to the root cause of the problem from both internal and external perspectives, and address any variations in service standards so sustainable benefits flow over the long term.

\section{Employee Turnover}

Organisation A's ongoing high employee turnover is an important factor impacting provision of training, as it triggers a continuous need to train large numbers of new recruits in a wide range of domains and processes. Its employee turnover rate is very high in business lines such as voice processes, and those involving night shifts. It is high for Band 5, and less high for Band 4 employees. On an average, it ranges between 15 and $20 \%$ per month. This translates into a significant challenge for Organisation A. One Black Belt—Training noted:

Focus, emphasis and challenges change, because you are talking here of upskilling about 1000 employees per month in different domains and verticals. For example, you are not talking of people being trained in a narrow range; people are upskilled in finance, insurance, IT, collection, customer service skills, and voice businesses. They are all different. 
The situation is compounded by the high growth rates in the ITeS/BPO sector, which create a demand from smaller and less established service providers for experienced and skilled employees. With growth opportunities, poaching becomes a characteristic feature of the external labour market. New players in the BPO/ITeS sector prefer to hire people from the existing players in the market, owing to a range of factors, including: a shorter ramp-up time for delivering their projects, an inability to get appropriately skilled people from existing educational institutions, and their inability or unwillingness to invest in a training and development infrastructure.

Most respondents felt that a significant number of employees consider their employment as a source of quick pocket money and attributed employee turnover mostly to the naivety of relatively youthful cohorts with no sense of developing a career in the evolving value chain of BPO services. Despite high employee turnover, the organisation's investment in training is still critical, as summarised by two respondents:

\footnotetext{
We need to train people through education@work program as it is hard to find people in certain domains. Domain experts who are MCSE-certified are hard to find. The point is that we need to upskill our people. Why, because tomorrow when we need people in this field it is not easy to find MCSE certified people. Cross movement is fine, but the challenge is to be able to make them relate to contextual bit. (Black Belt_-Training)
}

The biggest challenge that comes here is retention. After a person has stabilised on his job, this is the time when we start thinking about his next move, in terms of self-development. We have clear defined learning paths for various people at various levels. This is also done as part of our regular evaluation process - performance and potential development process. We actually work with a level higher. (Process Head-Content Solutions).

The contextual bit here relates to "integrating" people into the metrics-driven culture and also understanding clients' contextual requirements.

\section{Enterprise Size, Complexity, and Ownership}

Contrary to what is noted in the literature, more than size, expressed as number of employees, it is the complexity of business operations at Organisation A that drives the nature and extent of training. Complexity here refers to the process tasks and the new roles established as a result of its expansion over the last seven years. The Process Head-Content Solutions noted: 
In fact, when we started off, the size of the service was small and the nature of product offerings was simple - one or two lines. Now we have got a diverse set of offerings, so the people have to be trained in those business lines. In addition, we have got more roles to be performed ... It has become more complex now.

As noted earlier under the section on workplace change, a change in the ownership pattern and the resultant change to a dual business model (captive and third-party) had a significant impact on the diversity and volume of training. Additionally, there is significant evidence of the quality-based culture of its parent organisation all through its operations.

\section{Temporal Dimension of Processes}

The emphases of training interventions differ at various stages (see Figure 14). To explain the variation in training within and between organisations - a problem currently identified in the extant literature - a project or process level understanding is critical. The temporal dimension of a process's life cycle explains the variation at various points of a process or project life cycle.

Whenever a new process is transitioned to India, the initial volume of training is high. It then tapers off, especially when the process delivery team has undergone process training, and starts delivering on the process. However, this pattern does not follow the traditional growth, stability, plateau, and decline life-cycle model. Instead, even after the initial bump in the extent (volume) of training, the training volume increases because of ongoing employee turnover and the process changes requested by the clients. The nature (diversity) of training, on the other hand, is likely to increase with changes to process specifications. Usually, short and informal refresher training captures such changes, but if the size of the requested change is large and complex, a formal training programme needs to be developed. The Process Head-Insurance commented:

There is a lot of new information that comes in from client sites. Now, when all this information comes from client sites, this has to be communicated to all the people. It could be various things. For example, a client was using a specific form and has now moved to a new form of design. This has an impact on the training. 
Training demand is further caused when the organisation receives business processes from different clients or even the same client but from one of its divisions in another geographical region, even if the process is for the same domain. This is explained in the following sub-section on geographical dimension.

\section{Geographical Dimension}

The diversity and volume of training requirements change as the organisation receives its business from different countries even if for the same domain and from its captive (parent) organisation's business. Typical training requirements include understanding the culture, language, legislation, and accents. This is of particular importance for voice businesses. Further, due to the strong regional mother-tongue-influence of agents in India, training is offered to neutralise the regional accents to serve different nations' or regions' accents. In the US, for example, an understanding of federal and 50 state government regulations is critical for delivering on processes such as insurance and accounting processes. Employees need to understand these federal and state regulations. Other reasons include the use of specific technologies that the organisation is not currently using at its delivery centre. In a third-party contract environment, which the organisation is entering into more, everything could be different.

\section{Specific Impact of Factors on the Nature and Extent of Training}

Having discussed the main factors that influence the nature and extent of training, the following section focuses on the relationships, intensity and direction between various factors and how these impact the nature (diversity, externalisation, transferability, and formalisation) and extent (volume) of training. In the following section, a conceptually clustered matrix was developed covering details of the main factors and the extent to which each factor appears to be present or lacking in this case (see Table 6). The relationships between factors were developed using explanatory maps for case organisations' feedback (Miles and Huberman, 1994).

\section{Extent of Training}

On average, the organisation invests in about 12 days of training per head per annum. Once the client specifications are sensed, disseminated and transferred to Organisation A, they are translated into demand for training. In newer service lines, training occurs even 
before the process is transitioned, when the business development or client-facing groups are developed for scouting business opportunities. High levels of $M O$ and $L O$ and a strong SS philosophy enable conversion of client specifications into training needs. It follows from the analysis above that $L O, M O$ and $S S$ have an influence on client specifications and consequently on training volume. In terms of temporal dimension, depending on the stage of a process life-cycle, employee turnover, and changes to client specifications, the impact on volume of training varies.

Similarly, depending on the size and complexity of the new process requested by clients, the volume of training is likely to change. However, the extent to which training is provided is moderated by the skill sets the organisation is able to procure through its HRM infrastructure. The QMS have a direct impact on the volume of training for all Band- 4 and above employees and an indirect impact through its interaction with its LO and MO capabilities. As noted, changes to strategy resulted in workplace change, which had a significant impact on the extent of training volume.

\section{Nature of Training}

Nature or diversity of training refers to all types of training and education that are provided in Organisation A. It includes technical and firm-specific, generic and transferable, internal and external, and formal and informal learning and development. Contrary to the basic tenets of the HCT, the organisation invests in both generic and transferable and technical and process-specific training. While process-specific training emanates from client specifications, there are some generic training modules, such as developing client-facing skills, project management capabilities, and domain-specific capabilities that are essential for responding to client needs. Further, given the organisation's high employee turnover rates and growth in business volumes, investment in training is used as a strategy to attract and retain employees, and contain its costs. This finding rivals the existing explanations of theoretical models of employee turnover.

Owing to its complex and multiple business lines, the training demands put on the training infrastructure are diverse. Strong MO, LO and SS capabilities are required to streamline the provision of such diverse training needs. Diversity of training is governed by the level of complexity of the business processes, which determines the levels of skills for hiring specifications. Depending on the level of qualification and experience match 
obtained through internal and external labour markets, additional training gaps are addressed. Diversity of training also increases when business processes are sourced from different geographical regions and/or when ongoing process changes are requested from the client end. Workplace change also adds to the diversity of training, as skills needed for a non-captive environment are very different to those needed for a captive environment. This was evident in the organisation's conscious attempt to move from a "captive-centric" language and way of doing business, to a more "market savvy language” and becoming a "commercially-savvy" organisation.

Reliance on external training and education providers is noted for certain complex processes, where internal expertise in not well developed and skills are hard to find in the external labour market. Examples include: qualified instructional designers, PMI-certified resources, and CPAs. Through its education@work programme, employees study towards various national and international qualifications.

To partially overcome recruitment and retention challenges, the organisation engages external training providers for pre-hire voice training for potential employees. Such investments are part of its overall SHRM approach and help the organisation in developing "the best in class" and "employer of choice" brand image. The education@work programme and Organisation A University infrastructure, have more recently attracted a relatively large and untapped pool of part-timers for certain low-end processes. While this helps in managing its human resource flow, service costs, and employee turnover issues, it is also a departure from the extant literature on investment in training for non-standard employees (Hall et al., 2000; VandelHeuevel \& Wooden, 1999). External training providers have also been used to develop specialist "commercial" and "market savvy" skills for its client-facing groups.

Formalisation of training practices and development of a formal infrastructure for identifying, designing, developing, and evaluating training are understandable for a diverse and complex organisation of this size. The organisation's high levels of MO, LO, and a SS-DNA also help in formalisation of training activities. Further, its HR infrastructure allows for formally maintaining an inventory of skills and competencies and the proficiency levels for each role-specific competency. Certain types of training, such as client-specific and process training, lend themselves to formal delivery and assessment. Other training that lend itself to formal assessment and certification includes: train the trainer (TTT), SS certifications (black belts and green belts), and LOMA 
certification. Further, training for certain high-end, detailed, and complex processes is such that it can be better delivered in a formal and structured learning environment. Given the focus on continuous process improvements and the use of dashboards and metrics, formal training deliverables are developed for most processes.

Table 6.

Conceptually Clustered Matrix of Factors

\begin{tabular}{|c|c|c|c|c|}
\hline S.No. & Main factors and attributes & Intensity & Respondents & Researcher's notes \\
\hline 1 & $\begin{array}{l}\text { Workplace change } \\
\text {-structural } \\
\text {-ownership } \\
\text {-technological }\end{array}$ & $\begin{array}{l}\mathrm{H} \\
\mathrm{H} \\
\mathrm{M}\end{array}$ & $\begin{array}{l}1,4,5 \\
1,2,4,5 \\
1,4,5\end{array}$ & $\begin{array}{l}\text { Post ownership further changes to structure, systems and } \\
\text { technology followed }\end{array}$ \\
\hline 2 & $\begin{array}{l}\text { Market orientation(M0) } \\
\text {-information sensing } \\
\text {-information dissemination } \\
\text {-organisational response }\end{array}$ & $\begin{array}{l}\mathrm{H} \\
\mathrm{H} \\
\mathrm{H}\end{array}$ & $\begin{array}{l}1,4,5,6 \\
1,2,4,5 \\
1,2,4,5,6\end{array}$ & $\begin{array}{l}\text { Very high levels of M0, coupled with high levels of } \mathrm{LO} \text { and } \\
\text { SS appear to have synergistic effects on training responses }\end{array}$ \\
\hline 3 & $\begin{array}{l}\text { Learning orientation(L0) } \\
\text {-commitment to learning } \\
\text {-open-mindedness } \\
\text {-shared organisational vision }\end{array}$ & $\begin{array}{l}\mathrm{H} \\
\mathrm{H} \\
\mathrm{H}\end{array}$ & $\begin{array}{l}1,4,5 \\
4,5,6 \\
1,4,5\end{array}$ & $\begin{array}{l}\text { Well-developed forums, processes and SS tools enable high } \\
\text { levels of LO }\end{array}$ \\
\hline 4 & $\begin{array}{l}\text { Quality management systems (QMS) } \\
\text { - Commitment \& sharing information } \\
\text {-continuous improvement } \\
\text {-teamwork }\end{array}$ & $\begin{array}{l}\mathrm{H} \\
\mathrm{H} \\
\mathrm{H}\end{array}$ & $\begin{array}{l}1,2,4,5,6 \\
1-6 \\
1-5\end{array}$ & $\begin{array}{l}\text { Organisation A's path dependence, heritage, and captive } \\
\text { culture are driving its strong SS-DNA }\end{array}$ \\
\hline 5 & Employee turnover & $\begin{array}{l}\text { H to M to } \\
\text { L }\end{array}$ & $1-5$ & Process dependent e.g. voice $=H$ and back-office $=\mathrm{M}$ to $\mathrm{L}$ \\
\hline 6 & $\begin{array}{l}\text { Enterprise size, complexity and } \\
\text { ownership }\end{array}$ & $\begin{array}{l}\mathrm{H}, \mathrm{M}-\mathrm{H} \& \\
\mathrm{MNC}\end{array}$ & $1-5$ & $\begin{array}{l}\text { Large and very complex: numerous verticals and domains. } \\
\text { High impact for non-captive environments }\end{array}$ \\
\hline 7 & Temporal dimension & $\mathrm{H}$ & $1,2,4,5$ & $\begin{array}{l}\text { Each new process or change in client specification has an } \\
\text { impact on training }\end{array}$ \\
\hline 8 & Geographical dimension & $\mathrm{H}$ & $1,2,4,5$ & High for captive and very high for non-captive environments \\
\hline 9 & $\begin{array}{l}\text { Strategic HRM approach and workforce } \\
\text { composition } \\
\text {-rewards and performance management systems } \\
\text {-career planning } \\
\text {-recruitment skills level \& operating roles; and } \\
\text {-use of non-standard employment }\end{array}$ & $\begin{array}{l}\mathrm{H} \\
\mathrm{H} \\
\mathrm{H} \\
\mathrm{H} \\
\mathrm{L}\end{array}$ & $\begin{array}{l}1,3,4,5 \\
1-5 \\
1,3,4,5 \\
1,3,5 \\
1,3,4,5\end{array}$ & $\begin{array}{l}\text { Cross-skilling for career progression; opportunities to } \\
\text { demonstrate capability supported by appropriate reward and } \\
\text { performance management systems. Use of part-timers limited }\end{array}$ \\
\hline
\end{tabular}

Legend: $H$ =High; $M=$ Medium; $L=L o w ; N=N i l$ 
However, certain behavioural training, does not lend itself towards formal evaluation, as compared to technical training, because it is dependent on the individual's willingness to internalise behaviours espoused by the organisation. Legislation and compliance training is also formally assessed and delivered. Typically, employees have to familiarise themselves with different laws and regulations, and tested for their understanding before they are allowed to work on the process.

\section{Conclusion}

The above analysis identifies the impact of factors influencing the nature and extent of training. It also points to the conditions under which variation in training happens. What follows from the above analysis is that the synergistic effects of strong levels of $M O, L O$ and a SS-DNA are strengthening the organisation's daily routines and helping it to develop a strong client-specific capability. Such a capability has been developed over a long period of time through repeated interactions between the clients and the service provider. The nature of long-term contracts and a captive environment supported such development. The nature of this capability is likely to be developed differently to accommodate third-party BPO work.

Other capabilities that the organisation has developed through the synergistic effects of $\mathrm{MO}, \mathrm{LO}$, and SS, and a strategic approach to HRM are domain and process expertise and project management capabilities. Again, these appear to have evolved over a long period of time.

Size, which on its own is a complex factor and explains limited variance in training, is better explained when analysed along with complexity of business processes and how the production function is organised: mass-production, slightly differentiated, or a highly differentiated (Boxall, 2003; Wilkinson, 2002). Complexity is evident in the organisation's highly differentiated services strategy. Strong QMS appear to support managerial control over process and work organisation. The within-case analysis findings reject the basic tenets of human capital theory (Becker, 1964). Within-case analysis points to investment in both job-specific and generic skills and education programmes, despite medium-to-high levels of employee turnover. Such investment is due not to asymmetrical market information (Acemoglu \& Pischke, 1998a, b), but to the dynamics 
of the external labour market and the lack of specific skills needed by a fast- growing industry.

Finally, in contradiction to using an increasing number of non-standard workers for developing workforce flexibility (Boxall \& Purcell, 2003), a high incidence of standardised forms of employment was noted. Finally, even where there were nonstandard forms of employment, contrary to the extant literature (Hall et al, 2000; VandenHuevel \& Wooden, 1999) access to training opportunities by such workers was not an issue. 


\section{CHAPTER 5.2}

\section{ORGANISATION B}

\section{About Organisation B}

Organisation B is a diversified, third-party Indian service provider, specialising in networking technologies and business process outsourcing (BPO) services. Established in the early 1990s, this Mumbai-based organisation employs about 1500 people across its IT-managed services, enterprise resource planning (ERP), security, and BPO divisions. This case study focuses on the BPO services division, which employs 900 employees working in two main business lines: voice operations and back-office operations. Voice operations are split into inbound and outbound call centres catering to the telecom, insurance, and finance industry sectors. Its back office covers legal transcription and financial knowledge process outsourcing (KPO) services, mostly for UK- and US-based clients.

Given the multi-case, embedded case study design, both voice (outbound sales) and its KPO business lines were studied in detail. Access was provided to a number of organisational documents and to published information about its HR policies, training, and quality management processes. The researcher had the opportunity to observe the daily routines of employees in their work settings. The main respondents include: the Head of Human Resources, business process heads of Voice and KPO, team leaders (TLs), a voice and accent trainer, and a few process employees.

\section{Organisation Structure and Processes}

The organisation's voice and KPO teams are structured around client processes and products. Organisation B started with dedicated fixed-price and commission-based outbound sales contact centres for its clients. Under this business model, its employee strength grew to 3000 . However, this business model was not sustainable because there was no scope for organisational capability development, value-added services, or opportunities to bring about productivity improvements, and all its services were restricted to selling products. Further, with increasing labour costs and declining billing rates, the organisation had to restructure its operations. In 2003/04, the organisation made 
a strategic decision to move from fixed-price commission-based contracts to time and material contracts and added differentiated services to its portfolio. This decision led to a significant reduction in its workforce. In 2004/05, it added KPO service lines.

Work is organised around Taylorist principles for its voice process. Its outbound sales team has different sales campaigns, such as credit cards and telecom products for US and UK clients. Specifications from the US clients are stringent and require exacting performances from the call centre agents. For example, one US client required a threshold of $85-90 \%$ for test results from all voice and accent trainees. Use of a verbatim sales script, dissemination of precise product information, and compliance with relevant legislation are further examples of high prescriptive standards. Relative to the US clients, the UK clients allowed the agents flexibility, as long as the service quality wasn't compromised, and changes to SLAs increased sales.

Its KPO service provides the sustainability ratings of large multinational corporations to a UK-based client. The above work is akin to Standard and Poor's or Moody's sustainability ratings of large organisations. Such ratings are often used by investment firms and pension funds to guide their investment decision making. The nature of work undertaken in this service line typifies highly differentiated high-end knowledge work (Boxall, 2003). A total of 90 employees are working in four teams organised around industry clusters. These industry clusters are energy (for example oil, coal mining, and power generation), utility (for example electricity, water, transport, telecommunications), health (for example bio-technology, pharmaceuticals, healthcare, consumer care), and technology (for example media, information and communication technologies).

The industry cluster approach was developed by the proprietary rating model of Organisation B's client. The rationale for clustering certain industry sectors together is that firms in certain clusters, such as energy, would have a similar impact on environmental activities. For example, BHP in mining and Shell in oil refining have some common environmental and human rights concerns. Similarly, firms in the health cluster face common ethical issues.

Each team in its KPO line is supported by a team leader, two quality leads and about 15 to 20 associates for each industry cluster. Work organisation and reporting relationships are informal in nature. The team and quality leads play the role of informal mentors and 
coaches for new recruits. The above work organisation is in stark contrast to its voice operations, where the team leader and quality leads have a much more formal role, involving tight control and monitoring of workflow, typical in a Taylorist, mass production environment.

\section{Business Processes, Strategy, and Work Organisation}

The business development managers (BDMs) are based out of the US and the UK and are supported by a pre-sales team from India, which generates leads from client databases and serves as a information hub to potential clients and competitors. Any lead that is developed through BDMs is sent to the transition managers, who, together with the BDMs, contact clients to offer solutions and feasibility reports for a given process. Depending on the complexity of the process, the solutions development could take up to 30 days. Following the solutioning process, SLAs are negotiated with clients, and the operations team from India takes ownership of the process to commence the pilot stage. The operations team decides on people needs, infrastructure and technology requirements. At the end of two months, the process completes the pilot stage and goes live.

Up to $60 \%$ of the recruitments in the voice business line have $0-9$ months work experience and another $20 \%$ have $9-18$ months work experience. Thus, with $80 \%$ of its workforce having less than 2 years of work experience, the organisation strategically invested in building a training infrastructure to offset the high wage costs involved in recruiting people with experience. For its KPO services, it recruits graduates who have some understanding of critical management and governance issues affecting large MNCs. The profile of its KPO intake includes accounting graduates, MBAs, and chartered accountants. The HR strategy has to offer compensation that is competitive and comparable with that of large financial services firms such as American Express.

Its KPO service uses a proprietary model developed by its client and contains 278 parameters, on which data is collated and analysed for calculating sustainability ratings. Depending on the nature of reports required, different regression equations are run for each ranking. Findings are compared with those of firms in an industry cluster. The final report states the assumptions, reservations, and limitations that apply to the ratings and conclusions. 
The organisation made a strategic decision in 2003/04 to move away from selling standardised, commission-based telecom, insurance and financial services products to a mass-product market to offering differentiated services with a range of contractual arrangements. The decision resulted in a major restructuring exercise, and moving into KPO and legal transcription services consequently required further investment in its HR, training, and quality management infrastructures. The decision to move up the value chain in BPO services was timely, because domestic and international competition for voice services was intensifying and numerous overseas organisations were setting up captive centres in India. Further, the billing rates in KPO services (especially for developing sustainability ratings) are much higher and competition is relatively less.

Differentiated services require a differentiated HR infrastructure. Each service line has different recruitment and selection strategies to achieve a better strategic fit between employee skills, client specifications, and the organisation's need to manage its workflow better. For example, in KPO services, all short-listed applicants needed to undergo a battery of written and analytical reasoning tests to assess their technical, statistical, accounting, and soft skills. The soft skills are tested using profiling inventories such as DISC profiling, which focuses on assessing personality traits such as Dominance, Influence, Steadiness, and Compliance.

Similarly, different performance management systems are designed for different service lines and job roles. For example, in voice services, agents undergo a monthly review of performance using a ranking scale. Team leaders are appraised using key result areas and business quotas. Process and function heads follow a modified balance scorecard approach to appraisals. KPO team members follow differentiated performance management systems depending on the industry cluster to which they belong. For example, an employee covering analysis for oil companies would need to cover thousands of published documents, unlike someone working in a technology firm such as Google or Yahoo. The focus of each appraisal system reflects its strategic and operational needs. Consequently, it affects how performance and training needs are identified.

\section{Nature and Extent of Training}

The nature and extent of training provided varies with each process (see Figure 15 below). On average, each year the organisation spends an equivalent of $5 \%$ of its 
revenues on training, which includes induction and ongoing training and development activities. The nature of training provided is diverse and varies for each business line.

\begin{tabular}{|c|c|c|c|c|c|c|}
\hline $\begin{array}{l}\text { New hire \& } \\
\text { voice \& accent } \\
\text { training }\end{array}$ & \multicolumn{2}{|c|}{$\begin{array}{l}\text { Process \& } \\
\text { domain-specific } \\
\text { training }\end{array}$} & \multicolumn{2}{|c|}{$\begin{array}{l}\text { Refresher } \\
\text { training }\end{array}$} & $\begin{array}{l}\text { Team leader } \\
\text { training \& } \\
\text { development }\end{array}$ & \multirow{2}{*}{ 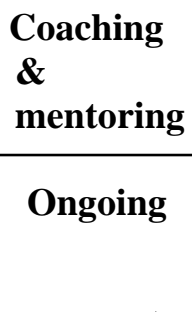 } \\
\hline $\begin{array}{l}2-3 \text { days } \\
7-10 \text { days }\end{array}$ & \multicolumn{2}{|c|}{$\begin{array}{l}\text { 1-10 weeks } \\
\text { (process dependent) }\end{array}$} & \multicolumn{2}{|c|}{$\begin{array}{l}\text { Process/Product } \\
\text { enhancement } \\
\text { Client-dependent }\end{array}$} & $\begin{array}{l}\text { External } \\
\text { provider- } \\
\text { dependent }\end{array}$ & \\
\hline $\begin{array}{l}\text { (TIMELINE DE } \\
\text { 2005-2006 key }\end{array}$ & $\begin{array}{l}\text { PENDENT C } \\
\text { focus areas }\end{array}$ & $\begin{array}{l}\text { N NATUI } \\
\text { frainin }\end{array}$ & $\overline{R E O}$ & PROCESS \& & COMPLEXI & $\Gamma \mathbf{Y})$ \\
\hline $\begin{array}{l}\text { Induction \& } \\
\text { voice \& accent }\end{array}$ & $\begin{array}{l}\text { Process } \\
\text { training }\end{array}$ & $\begin{array}{l}\text { Domair } \\
\text { knowle }\end{array}$ & & $\begin{array}{l}\text { Refresher } \\
\text { training }\end{array}$ & $\begin{array}{l}\text { Developing } \\
\text { SMEs }\end{array}$ & $\begin{array}{l}\text { Quality } \\
\text { standards }\end{array}$ \\
\hline
\end{tabular}

Figure 15. Temporal dimension of training: Changing emphases.

For example, in outbound voice services, induction training starts after an applicant passes the voice and accent test. Quality management standards (of COPC-Customer Operations Performance Centre) require a minimum level of skill for new applicants. The applicant then undergoes basic grammar, computer skills, and customer relationship management training. Depending on the test results, the employee is allocated to an inbound or outbound project and process-specific training follows.

For UK and US processes, separate country-specific voice and accent training is provided. For US processes, stringent process compliance and client specifications require about 10 days of training, compared to 7 days for UK processes. Because of its high volume of voice services, as well as employing internal voice and accent trainers, it contracts external native English speakers from the UK and US.

Process-specific training in voice services varies from 7 to 20 days in length for UK and US processes respectively. On completion of process-specific training, employees undergo a formal assessment and are allowed to work in operations only if they achieve client-stipulated thresholds, which vary from $70-90 \%$. In KPO, the training lasts for $2-3$ months and is divided into soft skills and technical or process-specific skills. While most training is provided informally and performed on the job by a buddy or a mentor, some training is structured and formal in nature. Organisation B's process manager reported: 
We take people through ... may be a detailed access of each and every parameter, each and every question, a large number of words that we have in the model, the whole glossary of terms, keywords, questions ... typically, what does senior management mean? If I am talking about senior management of say British Petroleum, a VP in British Petroleum is probably not senior management... What is a gentleman's agreement? What are the Equator Principles? What is a golden parachute? Those kinds of terms. Then we take them through the client's database, the property databases that we have access to, in terms [of] how to navigate them, how do you fill in the data, what is to be done, how it is sent, how does it come. Then we look at these kind[s] of questions that they need to answer, where do they find the data, the SEC site, Google, Prince of Wales Business Leaders Forum-which has a human rights watch - or maybe ILO website or Greenpeace website.

The extent to which the above training is provided depends on the complexity of the process and the industry cluster. Based on the employees' understanding, quality checks are done on the work that they produce. Buddies informally provide feedback on what is lacking or on how additional data points could have been covered. As noted by the KPO process manager:

Analyst is somebody who has probably spent 8-10 years, and probably 1-2 years in life working as generalist, and has looked at the sector and he understands it.... Talking about technology companies: How are they generally structured? How does their board [structure] looks like? How does their financial statement look like? What can their activities be typically? ... If this happens ... how will it affect us ... .So that kind of knowledge you can only experience; nobody can teach you.

\section{Analysis of Factors Influencing Provision of Training}

\section{Workplace Change}

The organisation underwent changes to its business portfolio, from a product to a services environment in 2003/04. As noted earlier, enabling functions such as HR, training, and quality management were changed to support and implement the change in its business processes. The above change resulted in the development of new people-management capabilities and a further expansion of its business portfolio. Consequently, it translated into new training demands, although, at the time of this study, training demand from the above changes had stabilised. The Vice-President HR commented: "It's like changing the 
mindset as well as learning the new process."

\section{Quality Management Systems (QMS)}

The organisation has to its credit numerous quality management accreditations. For example, it has COPC certification, CMM, Six Sigma, and ISO certification as its key quality management systems for different business units. To deliver on the above quality standards, it has two levels of quality infrastructure: a centralised pool of quality management resources and a decentralised team that is aligned to various business processes. The centralised quality team advises on the strategic and marketing benefits of implementing quality to bring about organisation-wide process efficiencies.

QMS requires training provision on aspects of quality standards, six sigma methodologies, and the application of various techniques in implementing process improvement projects. Quality management resources are also deployed in various processes for identification of performance and training needs on an ongoing basis, thus influencing the formalisation and evaluation of the training provided. QMS enable the solutioning teams to negotiate with clients on the metrics for service deliverables.

The extent to which its QMS have developed expertise for various processes determines the extent to which they can influence its LO and MO, and consequently the process specifications. LO, MO, and QMS interact with one another at various stages of process delivery. Organisation B's internal quality standards are used as a reference point for internal process efficiencies and may or may not be specifically used for clients' requirements. However, they do provide a strong basis for the development of client SLAs and ongoing process improvements. While its LO and MO ability may capture and disseminate the agreed process metrics, the errors or defects can be calculated and improved upon only when the process reaches the go-live stage and if the clients are willing to accept process improvements and any associated costs. At this stage, the team leaders and quality analysts focus on process improvements, keeping in mind client specifications and the organisation's ability to engage in open-mindedness. Its QMS are limited by its inability to develop and disseminate its shared vision about process improvements and to influence clients to accept higher levels of service delivery. 


\section{Market Orientation}

MO also reflected the move from a product to a services mindset. Consequently, the focus was on developing a strong level of service orientation. Organisation B has medium to high levels of MO ability. Its information sensing and dissemination is distributed between its pre-sales team in India and the BDMs located in the US and the UK. Information sensing ability is evident in lead generation for BDMs and in catering to requests for information (RFI) and proposals (RFPs) from prospective clients. Dissemination of information and framing of responses takes place in conjunction with the transitioning and operations teams in India and overseas. Given the diversity and complexity of various services and clients' unique specifications in their SLAs, the solutions framed for each client are different. This impacts variously on the training provision, as noted by the Process Manager-Voice.

\footnotetext{
If you see our US process, $100 \%$ focus is on the script because that's provided by the client. If you see a UK process we may have some generic training also. So that really depends on the client's involvement. So clients may be very specific; this is the kind of training. And some clients may not be that stringent on the kind of training which we give. It really depends on the client. Some clients are very strict and they may come down and do the training for an initial period, and some other clients, they don't come in and they are not that much involved.
}

Of critical value here is the quality of information sensed from clients and how well it is disseminated to the operations team, as it determines the quality of the responses framed.

\section{Learning Orientation}

The impact of MO on the provision of training is also influenced by its LO, specifically its ability to challenge its "theory in use" (Argyris \& Schon, 1978). There was some degree of open-mindedness evident in the daily routines of KPO services. Organisation B's ability to challenge clients' assumptions and business routines was limited in the voice service line, as most US clients were unwilling to implement any changes to their agreed SLAs. Moreover, given the nature of employees recruited in voice-relatively young and inexperienced employees - the frequency and intensity of process improvements was less likely. Only when clients' sales targets were not being met or when an element of an ethnocentric approach was not effective did the US clients incorporate suggestions. As noted by the Process Manager-Voice: 
We do give script suggestions, but that's more to do with the selling of the product. So, for example, there are certain standards which don't work out or when the agent is making calls for one year, they definitely know which part of the steps works. So there are lots of script suggestions, which go out from us to QAs (Quality Analysts) because they are listening to the call, in and out. And most of the time, the compliance side for US is very strict. But they still try to be very cooperative and there have been many changes in the script, which has really helped us.

Commitment to learning is evident in the nature and extent of training and development opportunities provided. Although the organisation had set up functional and organisational forums, such as monthly open houses, cross-functional team forums, COO and $C E O$ forums for raising and addressing business problems, such information sharing is limited and, thus the ability of the organisation to develop a shared vision. Consequently, it has a medium level of LO.

LO and MO interact at different stages of the process. The organisation's openmindedness is stronger at the information sensing and response stage than during dissemination and is enabled by the quality management systems that it follows. The extent to which the organisation can standardise its training is affected by its quality management systems' ability to simplify clients' processes.

\section{Employee Turnover}

Compared to its KPO, the employee turnover rates in voice services are extremely high. In voice, it ranges between 70 and $80 \%$ per annum, whereas it is about $15 \%$ in KPO. Increase in the number of players in the ITeS-BPO market increases job opportunities for employees and results in poaching of experienced personnel by small start-ups. Poaching is more prevalent in call centres than KPO, and is likely to intensify in KPO firms as more players move up the ITeS-BPO services value chain. High employee turnover has a huge impact on Organisation B's training volume and infrastructure because the bulk of its employee turnover occurs within the group with less than 3 years experience. One process trainer commented:

That actually kills ... especially the trainers, because you put so much effort, and after one week, two weeks, when you go on the floor you won't find the entire batch. That really kills 
us.... Once you get a new agent, once again the cycle begins and they will take some time to absorb.

\section{Enterprise Size, Complexity, and Ownership}

It is not the growth or contraction in enterprise size that directly affects the nature and extent of training; instead, it is the complexity of processes in its portfolio of services that impacts on the overall provision of training. A process manager commented:

\footnotetext{
Because earlier we just had one or two UK processes. But now, if you see, we have KPOs, we have back office, we have legal transcription. So there are so many training going on which may not only be at agent level; it may be at supervisory level; it may be at managerial level. So that has increased a lot."
}

Although Organisation B had the challenge of developing its service credibility in a global industry, as an Indian-owned and operated third-party service organisation, its business model reflected the need to develop a training infrastructure as an integral part of operations and to contain the rising wage costs.

\section{Temporal Dimension of Processes}

The extent to which a new process or process enhancement impacts on the nature and extent of training also depends on the experience of a given process because the demand for skilled employees declines with the increase in experience on that process (Bartel \& Lichtenberg, 1987). The relationship between the temporal dimension of a process and the demand for skills is not simple in dynamic and turbulent environments. The analysis revealed that the demand for training increases with changes to clients' specifications and high employee turnover. Depending on the scope of changes requested, additional training is provided. As commented by the Process Leader-Voice, "It may be a quality update or refresher training. If it's a very small update, a QA or TL can take it as an update. But if it's a new offer itself, then we may have refresher training."

The nature of change requested by clients varies from software updates, through product knowledge, to working on revised quality metrics. High levels of employee turnover reduce the extent to which new knowledge can be assimilated by the team. Thus, the findings depart from the previously noted neo-human capital theories. 


\section{Geographical Dimension}

As Organisation B's clients are from the US and the UK, the differences in language usage, cultures, legislation, and client specifications require very different sets of training, even for a similar process. For example, its US-based processes require more training as its clients' expectations of performance are higher than UK-based processes do. Further, in its KPO, business processes require agents to develop a comprehensive understanding of geopolitical issues, applicable tax, accounting, and environmental legislation that affects multinational firms operating in different parts of the world. Understanding legislation of such order places significant demands on Organisation B's learning and development needs, as the external labour market does not have a well-developed skills market for this line of service.

\section{Strategic HRM Approach, Skills Level, and Workforce Composition}

As noted above, Organisation B's HRM practices are well developed and strategically aligned for both the divisions. Differentiated HRM practices exist for each of its business lines. Analysis of HR documents and annual reports suggests it has a well-developed internal labour market. Its workforce composition is predominantly (95\%) full-time, permanent process staff, and the remaining $(5 \%)$, also permanent, are in enabling functions such as HR, Marketing, and Finance. Its recruitment and selection strategies in voice services focus on acquiring employees with entry level skills and qualifications to contain costs and minimise employee turnover, because $80 \%$ of its workforce model comprises employees with less than 24 months of work experience. Because the labour market for people with analytical and consulting skills is not well developed, for KPO services it recruits people with higher qualifications, such as an MBA, a CA, or an M.Com.

\section{Analysis of Factors Influencing Provision of Training}

The following section provides an analysis of how various factors impact on the nature (externalisation, diversity, transferability, and formalisation) and extent (volume) of training. Following Miles and Huberman (1994), a conceptually clustered matrix was developed (See Table 7). 


\section{Extent of Training}

Training volume is directly affected by client specifications, process complexity, temporal dimension of processes, QMS, employee turnover, and workplace change. Workplace change, as noted above, impacts on both the volume and the diversity of training as it required a change to a service mindset and learning new business processes. Process complexity determines client specifications, which, in turn, influences not only the skills needed, but also the volume of training to be provided. Each new process introduced by the organisation increases the volume of training.

High employee turnover and clients' process enhancement requests impact positively on training volume. Investment in QMS impacts directly and indirectly on the nature and extent of training. Quality management standards require training for quality and key operations staff. Its quality management capabilities also affect its HRM practices, LO, and $\mathrm{MO}$, as it helps in the development of tangible service metrics for various process. While MO enables capturing of client specifications and disseminates it to the operations team, training on client relationship management and quality tools and techniques is required for client-facing groups.

\section{Nature of Training}

The organisation provides a wide range of soft skills and technical training. Although the focus is more on technical training (for example, process and domain-specific, clients' software applications, accounting standards, tax legislation, and industry-specific legislation), other generic training focuses on customer relationship management, communication skills, and voice and accent. The diversity of training is influenced by process complexity, quality management systems, and the temporal and geographical dimensions of processes. Relative to voice services, the organisation's KPO service relies less on external training providers because most of its training needs are met internally through informal and on-the-job training. Training diversity increases when a new client process is introduced or if the process is from a new geographical location. Legislation, language complexity, and cultural differences form the basis of such training needs. Changes requested by clients for enhancing the scope of a process often result in a new type of training. 
Its QMS and client specifications require formal processes to assess, design, and deliver the training provided. Client specifications drive formalisation as most US processes require assessment of employees following the training episode. Thus, MO capabilities, QMS, and performance management systems shape formalisation of training.

Table 7.

Conceptually Clustered Matrix of Factors

\begin{tabular}{|c|c|c|c|c|}
\hline S.No. & Main factors and attributes & Intensity & Respondents & Researcher's notes \\
\hline 1 & $\begin{array}{l}\text { Workplace change } \\
\text {-structural } \\
\text {-ownership } \\
\text {-technological }\end{array}$ & $\begin{array}{l}\mathrm{H} \\
\mathrm{N} \\
\mathrm{H}\end{array}$ & $\begin{array}{l}1,2,3,4 \\
1,2,4 \\
1,2,3,4\end{array}$ & $\begin{array}{l}\text { Post-restructuring changes to } \mathrm{HR} \text {, training and quality } \\
\text { infrastructure. High service orientation. }\end{array}$ \\
\hline 2 & $\begin{array}{l}\text { Market orientation (M0) } \\
\text {-information sensing } \\
\text {-information dissemination } \\
\text {-organisational response }\end{array}$ & $\begin{array}{l}\mathrm{M}-\mathrm{H} \\
\mathrm{H} \\
\mathrm{M}\end{array}$ & $\begin{array}{l}1,2,3,4,5 \\
1,2,4 \\
2,3,4,5\end{array}$ & $\begin{array}{l}\text { Medium to high levels of M0. Response influenced by } \\
\text { client specifications and their willingness to change. }\end{array}$ \\
\hline 3 & $\begin{array}{l}\text { Learning orientation (L0) } \\
\text {-commitment to learning } \\
\text {-open-mindedness } \\
\text {-shared organisational vision }\end{array}$ & $\begin{array}{l}\mathrm{H} \\
\mathrm{M} \\
\mathrm{M}\end{array}$ & $\begin{array}{l}1,2,3,4,5,6 \\
1,2,3,4,5 \\
2,3,4,5\end{array}$ & $\begin{array}{l}\text { Evidence of critical thinking is present but is dependent on } \\
\text { clients' willingness to accept change. }\end{array}$ \\
\hline 4 & $\begin{array}{l}\text { Quality management systems (QMS) } \\
\text { - Commitment \& sharing information } \\
\text {-continuous improvement } \\
\text {-teamwork }\end{array}$ & $\begin{array}{l}\mathrm{M}-\mathrm{H} \\
\mathrm{M}-\mathrm{H} \\
\mathrm{M}\end{array}$ & $\begin{array}{l}2,3,4,5 \\
1,2,3,4,5 \\
2,3,4\end{array}$ & $\begin{array}{l}\text { Well-developed internal and external quality standards. } \\
\text { External quality metrics are negotiated with, and depend } \\
\text { on, client needs. }\end{array}$ \\
\hline 5 & Employee turnover & $\mathrm{H}$ and $\mathrm{L}$ & $1-4$ & Process dependent e.g. Voice $=\mathrm{H} ; \mathrm{BO}=\mathrm{M}$ to $\mathrm{L}$ \\
\hline 6 & $\begin{array}{l}\text { Enterprise size, complexity and } \\
\text { ownership }\end{array}$ & $\begin{array}{l}\mathrm{M}, \mathrm{M}-\mathrm{H}, \text { and } \\
\text { Indian }\end{array}$ & $\begin{array}{l}1,3,4 ; 1-4 ; \text { and } \\
2-4\end{array}$ & $\begin{array}{l}\text { Large organisation with diversified portfolio. Process } \\
\text { complexity demands varying levels of training investment. }\end{array}$ \\
\hline 7 & Temporal dimension & $\mathrm{H}$ & $1,2,3,4,5,6$ & $\begin{array}{l}\text { Each new process or process enhancement impacts on } \\
\text { training volume and diversity }\end{array}$ \\
\hline 8 & Geographical dimension & $\mathrm{H}$ & $2,3,4,5,6$ & Multiple US and UK processes impact severally. \\
\hline 9 & $\begin{array}{l}\text { Strategic HRM approach and workforce } \\
\text { composition } \\
\text {-rewards and performance management systems } \\
\text {-career planning } \\
\text {-recruitment skills level \& operating role; and } \\
\text {-use of non-standard employment }\end{array}$ & $\begin{array}{l}\mathrm{H} \\
\mathrm{M} \\
\mathrm{H} \\
\mathrm{N}\end{array}$ & $\begin{array}{l}1,2,3,4 \\
1,3,4,5 \\
1,3,4,5 \\
1,2,3,4\end{array}$ & $\begin{array}{l}\text { Well-developed and differentiated performance } \\
\text { management and rewards and recognition systems. } \\
\text { Opportunities for career progression evident within service } \\
\text { lines. All full-time permanent workforce, } 95 \% \text { in } \\
\text { operational roles. }\end{array}$ \\
\hline
\end{tabular}

Legend: $H=$ High; $M=$ Medium; $L=$ Low; $N=$ Nil 


\section{Discussion}

The above analysis shows that numerous factors influence the nature and extent of training. Organisation B's medium- to high-QMS and MO, and medium level of LO results in adaptive learning (Argyris \& Schon, 1978). Also evident in the above analysis is the link between how an organisation's QMS enables the development of its MO, LO and HRM capabilities to work synergistically to bring process efficiencies. Its ability to introduce a higher level or generative learning is likely with further development of its QMS and a high LO.

The relationship between high or complex process specifications and higher levels of training volume and diversity is not straightforward because process specifications are also affected by the maturity of the process and the employee turnover rate it experiences. Separation of training from its HR function suggests an inextricable link between training and operations. HR practices and quality management systems play a monitoring and enabling role in enabling service delivery and managing the organisation's cost structures. Its workforce composition in the voice service line suggests a deliberate HR strategy to employ less experienced employees in order to contain its costs and optimally utilise its training infrastructure. Finally, the case suggests gaps in the Indian educational infrastructure for certain BPO services.

\section{Conclusion}

In order to build a high level of process maturity and engage in higher order learning, Organisation B will need to invest more in its training and quality management infrastructures. Such an approach would also provide added value to its clients. There is a need, too, to engage with industry, academia, and industry associations (NASSCOM) in order to develop generic training modules and certifications relevant for the ITeS/BPO sector. Such training would result in cost savings for the organisation, thus freeing up organisational resources for the building of higher order capabilities and providing better value for its clients. 


\section{CHAPTER 5.3}

\section{ORGANISATION C}

\section{About Organisation C}

Organisation $\mathrm{C}$ is a UK-based BPO firm operating from India to cater to the real estate and telecom industry sectors. Established in 2004 by two Indian entrepreneurs with funding from two UK-based organisations, it employs about 250 employees working in two main business lines: accounts back-office for real-estate and voice operations for telecommunication services, mostly for UK-based clients. It also provides other supporting services, such as outsourcing consulting, IT infrastructure management, and HR and legal services to its back-office clients. Its voice operations comprise in-bound customer support and outbound sales for telecommunications and electricity products.

Given the multi-case, embedded case study design, both its voice and back-office business lines were studied in detail. Access was provided to a number of documents, published information about its training, and HRM processes and policies. Also provided was the opportunity to observe the daily routines of employees in their work settings. The main respondents include the chief operations officer, HR manager, business process heads, process team leaders (TLs), quality manager, and a number of process employees.

\section{Organisation Structure and Processes}

Organisation C's voice and back-office teams are structured on the basis of its clients' processes and products. There exists a well developed Taylorist hierarchy. For example, in one of its voice process team for a Telecom client, it has dedicated staff strength of 70 outbound sales agents selling broadband connections to its clients' UK-based customers. Their workflow is monitored by team leaders (TL), systems and quality managers, quality assurance (QA) supervisors, and a call-dialler supervisor.

In back-office operations, employees are further split into key back-office processes for each client. Work is organised around processes such as accounts payable and receivable, bank reconciliation, vendor invoicing, client invoicing, and accounting accruals. On average, there are 5-6 sub-processes for each process and each sub-process has 6-7 employees. Each sub-process has a subject matter expert (SME) who reports to a TL. 
SMEs are supported by two QA supervisors. All TLs report to the process manager, who reports to the head of the service.

\section{Business Processes, Strategy and Work Organisation}

The business development (BD) team is based in the UK and is supported by a marketing team from India. The Indian team generates leads from client databases and serves as an information hub for potential clients and competitors. The team also identifies the business processes that can be outsourced to India and passes the leads to the BD team in the UK. The UK team makes presentations to clients and offers free consulting services, such as process feasibility reports, which indicate any business process re-engineering that may happen before a business is transitioned.

Once a client expresses firm interest in outsourcing its processes, the transitioning team, which essentially comprises people from the BD team in the UK and the operations team from India, develops solutions and draft service level agreements (SLAs) in consultation with the clients. SLAs include details about client specifications, process metrics, such as turnaround times, and performance standards. The transitioning team undertakes detailed time-and-motion studies of the processes and assesses the key requirements for a process. People specifications, skill sets, infrastructure and technology requirements are determined. After sign-off, the operations team takes over the process ownership. The process is piloted and only when a steady state is achieved does it go live for production and delivery. Process complexity and the skills and experience of employees determine delivery capabilities. Its business development manager noted:

\footnotetext{
For a voice operation, usually there isn't that much of a requirement to go there [UK] and learn as the processes are not all that intricate. Product knowledge is the only reason why people might need to go. But, see, typically in a back-office you would have people using various CRM applications, systems, and a very elaborate set-up over there that has to be integrated here. So they are on a steep learning curve. Even product knowledge can be done without someone going to the client site, unless it is a very complicated technology integration, for which we will have to send someone. But that doesn't really happen too much here.... Having said that, training does happen; we have clients' trainers who come over and train our people in the product knowledge.
}

The organisation made a strategic decision by carving out a niche market for itself in the real estate vertical. Firstly, at its inception, there were no BPO providers catering to the 
real estate sector in India. Secondly, as a small start-up, it was difficult to compete with the large and established ITeS/BPO players in well-developed industry sectors. With a relatively simple portfolio of two services, its competitive strategy corresponds closely with Boxall's (2003) slightly differentiated services strategy.

Although Organisation C commenced its operations as a third-party service provider, in 2005, a large UK-based property management organisation acquired its ownership stake to build on Organisation C's capabilities in the real estate sector for its real estate business in the UK. Post-acquisition, the focus shifted to a dual business model (from a third-party business model to a captive and a third-party model). Its dual business model will affect expansion of its back-office operations in 2006, taking its total employee strength to about 350 plus by the end of 2006. The Process manager-Back-office noted, "In the back office operations we have close to 60 employees. We should be around 200 by the end of 2006."

\section{Nature and Extent of Training}

In terms of the volume of training provided, on average, every employee receives about 10-12 days of training per annum. The organisation provides common induction training of 2-4 days to all employees. This is followed by process-specific and domain-specific training, which lasts from 1-3 weeks. The HR Manager remarked:

\footnotetext{
Our training currently is more focused on processes, process knowledge, from the client perspective, and quality, and now that we have started off into the growth phase, we will be looking into the development of people. We have most of the training as on-the-job training and we do not currently have a separate training and development department.
}

The extent to which this training is provided depends on the size and complexity of the process. Until new hires demonstrate acceptable proficiency levels, they are required to undergo further process training. For F\&A back-office processes, process training is provided in client's customer relationship management (CRM) software and other software applications, and tax legislation and statutory compliance for accounting practices.

In voice processes, accent neutralisation training is provided by a native English language speaker from the UK. The focus is on reducing and neutralising the regional mother 
tongue influence of employees coming from different states in India. Training also includes familiarisation with the UK's dominant national culture and regional cultures, dialects, and accents, to enable better communication with customers. Contrary to the popular media reports and research on call centres, in this case setting, the SLAs for selling broadband connections do not require agents to use false or anglicised names.

\begin{tabular}{|lllll|}
\hline $\begin{array}{l}\text { New hire } \\
\text { training }\end{array}$ & $\begin{array}{l}\text { Process } \\
\text { \& domain-specific } \\
\text { training }\end{array}$ & $\begin{array}{l}\text { Refresher } \\
\text { training }\end{array}$ & $\begin{array}{l}\text { Team leader } \\
\text { training }\end{array}$ & $\begin{array}{l}\text { Coaching } \\
\text { \& } \\
\text { mentoring }\end{array}$ \\
\hline 2-3 days & 1-3 weeks & $\begin{array}{l}\text { Process/Product } \\
\text { enhancement- } \\
\text { dependent }\end{array}$ & $\begin{array}{l}\text { External } \\
\text { provider- } \\
\text { dependent }\end{array}$ & Ongoing \\
\hline (TIMELINE DEPENDENT ON COMPLEXITY OF THE PROCESS)
\end{tabular}

Figure 16: Temporal dimension of training: Changing emphases.

As shown in Figure 16, training diversity subsequently focuses on developing domainspecific and product/process-specific knowledge, for example, sales training and/or customer support for outbound and inbound processes respectively. Most back-office and voice training is provided in-house, with the exception of the training from the clients' site of a few subject matter experts (SMEs). SMEs in turn train and develop team leaders and agents. The organisation ensures that employees entrusted with the task of imparting training undergo a Train the Trainer (TTT) certification process, administered internally, thus suggesting a fairly formal approach to process-related training.

As shown in Figure 16, process training is followed by refresher training, which occurs as a result of any process/product enhancements requested by the client. This happens frequently for voice processes, but to a lesser extent for back-office processes. The extent to which refresher training is provided is determined by the size and complexity of the process enhancements requested. It could be a small pre-shift team huddle (to update the team on small changes requested), half-day refresher training, or a formal full-day training programme (for larger updates). 
As the organisation is just beginning to expand in size and offerings, it now feels the need to invest in generic and behavioural skills to groom supervisory and managerial staff, especially as its best and brightest are picked from the ranks to lead, supervise, or manage teams. Given its limited training infrastructure, it has decided to source externally an industry-accredited team leader training programme, developed by various ITeS/BPO providers in conjunction with NASSCOM, the industry's professional body.

\section{Analysis of Factors Influencing Provision of Training}

\section{Workplace Change}

Being a relatively new entity, Organisation C underwent a series of metamorphoses before gradually developing its process capabilities. In the main, it has faced two major workplace changes: adding new business lines and the move to a dual business model (captive and third-party provider). The Manager-HR commented:

\footnotetext{
See, earlier we started off in voice operations, so we had multiple voice businesses. Then a conscious decision was taken to move into back-office, so we reorganised and the entire management focus shifted to work in back-office though it would now be more of a balance between back-office and voice processes. We are now concentrating on the kind of voice processes that we get. This decision had a lot of impact on the kinds of skill sets the organisation needed and a lot of training eventually happened once we moved into backoffice operations.
}

The above suggests the links between its competitive strategy and training. The change to a dual business model translated into higher training volumes and diversity. Additional processes added to its existing portfolio and the need to familiarise with different software applications used by its third-party clients required further training for implementation and for integration with the Indian operations.

\section{Quality Management Systems (QMS)}

Six Sigma (SS) methodology is prevalent to assess and service the external and internal quality needs of its clients. SS is embedded right from pre-transition work to post-go live. Use of SS is more pronounced in voice processes than back-office. From an internal quality perspective, in addition to SS methodologies, Organisation C's Customer 
Operations Performance Centre (CPOC) certification (www.copc.com) helps it to increase process efficiencies by focusing on cost reduction and increased customer satisfaction.

In addition to the efficiency focus embedded in COPC gold standard, the organisation complements it with an ITeS/BPO variant of the Capability Maturity Model (CMM), developed by researchers at Carnegie Mellon University in the US. Although Organisation $\mathrm{C}$ is not formally assessed for this certification, it follows the key principles of the certification by recruiting a person with experience in this framework. Overall, the focus is not only on the compliance of process deliverables as per SLAs, but also on developing process maturity by enhancing its internal process efficiencies at various stages of a process's life-cycle.

The above approaches affect training provision at various levels. First, there is a need to train and certify people on SS methodology and COPC standards, for which Organisation $\mathrm{C}$ relies mainly on external training providers. Subsequently, the externally trained employees become training resources for other employees. The organisation's QMS also drive formalisation, externalisation, and evaluation of the training provided. Second, the performance gaps detected through SS and other quality management frameworks translates into coaching, mentoring and/or formal training initiatives. Third, at an individual level, the QAs, in conjunction with TLs, identify specific areas of dissatisfaction and performance gaps and arrange for refresher training. At this point, it is important to understand how the organisation's LO, MO and quality management systems interact with one another from the pre-transitioning to the delivery stage.

The organisation's internal measures of, and standards for, process efficiency may or may not be specific to a client's requirements; however, they are often used in developing a client's SLAs. While its LO and MO may be able to capture and agree upon certain process metrics, the sigma levels can be calculated and improved upon only when the process goes live. At this stage, process improvements are looked at and agents and TLs begin to look at process delivery critically. Open-mindedness also comes into play here. One process employee-Voice commented:

We had a lot of customer complaints from the customers, when they switched over from product $\mathrm{X}$ to $\mathrm{Y}$. The downtime was very high, people were without phone lines and the Internet. So that was affecting our sales. It's all word of mouth; if one person is not happy 
then it spreads to others.... We went and told our TLs about it. They spoke to the managers and the managers spoke to the clients. Then we had a programme wherein they offered a lot of publicity and gave out a lot of reasons to customers why they were getting late in installing the connections. ... They were not adequately resourced to handle the large volumes of $£ 1$ connections and so there were delays. In the end, the clients took it positively and increased their manpower ... now we don't have any such problems.

It appears that the quality management systems, LO, and MO are working synergistically. Not only are the process metrics defined and communicated and adhered to as per client specifications, an effort is made to improve the process for delivering greater efficiencies and reducing costs. Further, as service delivery is established, existing metrics are improvised and suggestions are made to the client. The extent to which its theory-in-use can be challenged and changed also depends on the opportunity for value addition and the client's perception of benefits realisation.

\section{Market Orientation}

Its MO ability is likely to change as it moves from a third-party to a dual business model. Organisation $\mathrm{C}$ has medium to high levels of MO ability. Its information sensing, dissemination and framing of response activities are functionally and geographically distributed between India and the UK. Its information sensing ability is evident in lead generation and routine requests for information (RFI) and requests for proposals (RFPs) from prospective clients. Dissemination and response framing is done by the solutioning teams in India and the UK. Its information-sensing ability currently maintains the strategic focus of exploring only certain industry segments and processes. When information gets disseminated to the operations and transitioning team, it has a direct impact on the training provided and the solutions presented. The Manager-HR noted:

They (the team) know the actual numbers of people, their skill sets, etc. People go to clients' sites and get trained, they learn the process and come back and train the employees here. It's the people in the operations team who get trained initially and then clients come from the UK and, if necessary, they also train.

Further, as each process within a business line is unique, the specifications, SLAs and solutions framed for each client are different. Such diversity in deliverables and metrics affects the training provision and resourcing, thus suggesting a nexus between operations and human resources management. The Business Development Manager noted: 
The report we submit to the company is based on the detailed analysis of the business and what we understand about it and how and where we can offer solutions.... If the client has a very haphazard way of dealing with its accounts, then we try and streamline it with the use of this application/tool. If the client already has another automated application, like an ERP or something, then we need to carry out some integration to adapt to it.

The degree of precision with which the organisation senses the information and disseminates it from clients to its operations team determines the quality of the responses framed, which affects the nature and extent of training provided. Although the organisation has over the last two years established a training framework for voice processes training, the extent to which such training can be standardised is limited by the complexity of the processes, the unique client specifications for new processes, and its QMS. The Manager_-Process Quality explained:

\footnotetext{
If you are involved in selling for $\mathrm{X}$ company a broadband connection and company $\mathrm{Y}$ comes up with a similar product of broadband, the basic product is going to be the same. But when you are engaging to represent the $\mathrm{X}$ brand and the $\mathrm{Y}$ brand, then what we have to take care [of] is the brand differentiation. For brand X the USPs could be different.... In this case you have to decide what kind of positioning does the brand has [sic] in the market and devise your training according to that.... So that is what will determine the training needs in both the cases.
}

\section{Learning Orientation}

The extent to which its QMS and MO affect training is also influenced by its learning culture and its ability to challenge its theory-in-use (Argyris \& Schon, 1978). As the organisation was in the second year of its operation, its ability to develop a shared vision and a commitment towards learning was still evolving. However, its size and the relatively few business lines have helped it to communicate information quickly and efficiently. Daily operations meetings, fortnightly employee committee meetings and feedback sessions cover a range of operational and routine matters. In addition to the above, there are quarterly feedback sessions with senior management to provide an update on any upcoming changes. The Process Manager-Voice noted, "There are a lot of levels and bureaucracy to be crossed in a large organisation as against a smaller one.... Yeah. Ideas here are accepted easily and heard more frequently, and ideas are implemented in a much faster way." 
The organisation demonstrates open-mindedness to the information, sensed and disseminated through its MO abilities. Usually, this happens prior to process transition to India, but, post-transition, open-mindedness is needed to bring about long-term process efficiencies. Employees challenge the clients' theory-in-use through its consulting and solutions teams, using its internal standards of performance. The business development manager noted:

\begin{abstract}
We provide them with the reengineered processes. It may be very similar to their current process, but certain aspects in the boxes in the flowchart may differ, or it can be a completely redesigned and reengineered process. So, yes, some suggestions are made. Then again the client may implement this right then or may do it later.
\end{abstract}

The ability to challenge clients' assumptions is somewhat limited for voice processes, as it involves selling a product, wherein agents have to comply with stringent product specifications, as set out in the SLA. Furthermore, the client may not be willing to bring changes to its product offering. However, opportunities exist to implement process efficiencies, which have an impact on training, as the HR manager explained:

From the point of view of making our lives easy, even if the client has not requested any process improvements, we do it....See, agents have been involved in these projects of improvements; whether with the QA or someone, the agents' involvement is there. They are given the opportunity to get involved. Again refresher training is also provided as needed.

\title{
Employee Turnover
}

Organisation C's employee turnover rate varies for both its service lines. In voice operations, employee turnover is high, averaging at about $80 \%$ per annum; whereas in back-office operations, it averages about $40 \%$ per annum. Further, in voice processes, employee turnover is mostly at agent and TL levels. Excessive employee control, imposing unreasonable targets, "lack of a good crowd", "naivety of agents", and quitting for higher incomes elsewhere are common reasons for quitting. A process manager-BackOffice added:

Now there's an increase in back-office turnover this year because of the high growth in back-office business in the industry.... In terms of voice, one thing is that the opportunity is available. Lots of call centres are available, and primarily the target group is the same. 
The impact of high levels of employee turnover is summarised by a team leader:

The attrition rate in voice business is so high, all across the industry, that there is a constant need for providing training to new hires. So, every few weeks we have some people here, so we have to train them. It's a bit different in back office ... you train them, they will stay for a reasonable period of time and only few will leave.

Owing to the above trend and the organisation's limited resource ability, it has not yet decided to commit to formal education and generic soft-skills training. Once its operations expand and its need for managerial and leadership skills increases, investment in generic skills is likely. For the above reasons, the organisation is focusing more on technical and process-related training.

\section{Enterprise Size, Complexity, and Ownership}

Change from Indian ownership to UK ownership will impact on the structure of the organisation's captive operations and the way different technology platforms and standard operating procedures followed in the UK are integrated into Indian operations. It will thus have an impact on the volume of training provided. In terms of enterprise size, it was the portfolio expansion from one to two business lines that resulted in an enhanced need for training. It is important to note here that the relationship between training and enterprise size is not a linear one; instead, it depends on the simplicity or complexity of the processes. The Manager-HR noted:

\footnotetext{
Some processes are very complex and would take about a month, whereas others are pretty easy and would take about 8-10 days. Again, with outbound sales we have different levels of processes and the time lines would vary. Depending on the product and the process complexity and the sort of skills that we have, the training times would vary.
}

\section{Temporal Dimension of Processes}

The extent to which a new process or process enhancement impacts on the nature and extent of training is determined by the experience gained on the process and the process maturity of the first batch of employees. However, as time elapses, the process team could re-look at the skill sets needed for future hiring (Bartel \& Lichtenberg, 1987). For example, as part of a client's SLA, a process required Telecom engineers for a technical support software applications process. However, once the process went live and the initial 
batch had had some exposure to the process, it was felt that the same process could be supported by people who had had experience in similar software applications, or simply had a knack for software applications, IT helpdesk, and troubleshooting roles. A quality manager noted:

\footnotetext{
In a BPO set-up, you know, everything that you have goes through a capability cycle....The life cycle of project depends on the complexity of the product/project. Initially, when the project is new, definitely the training needs are very, very high. And as and when the people involved are trained and a certain amount of maturity and experience develops, people are comfortable with the project, and the project is going into a mature stage, then definitely training needs at an individual level reduces[sic]. But overall then you would start focusing on how you would bring about continuous improvement into the process.
}

As the process matures, trainers refine training modules for subsequent batches and the volume of training should theoretically decline for subsequent batches. However, owing to high employee turnover rates, the frequency with which such training is offered increases. Process enhancements also necessitate training provision. Such demands could result in formal or refresher training, from simple changes like adding/deleting a few data fields in a process, through briefings on Christmas specials, to how a new product offered by a competitor affects the way agents should position their sales campaign. The impact of different geographical locations on the diversity of training is explained in the following section.

\section{Geographical Dimension}

Although Organisation $\mathrm{C}$ receives the bulk of its BPO work from the UK, earlier it had voice processes from the US, which required a very different set of training. US processes required training on voice and accent neutralisation for US conditions, understanding of US legislation, tax rules, culture, and their existing client-specific software applications. For UK processes, training needs differed because of its accounting principles, standards, clients' software applications, and certain key legislation that governed its real estate and telecommunications sectors.

To minimise some of its ongoing need to learn the multiple software of its clients, Organisation $\mathrm{C}$ entered into a strategic collaboration with a global software development company specialising in accounting software for the real estate sector, especially for the US and UK markets. It then negotiated with clients the use of such a standardised application, as it would increase process efficiencies for both parties and enable instant 
and effective client interface over the Internet. This aspect of dynamic coordination and strategic demand management echoes Banerjee's (2004) findings of Indian ITSS firms.

\section{Strategic HRM Approach, Skills Level, and Workforce Composition}

Organisation C's strategic approach to HRM practices is still nascent, although it has operational recruitment, performance management, compensation, and reward and recognition systems. As noted by a TL, "HR is more of a quality, policy, and documentation function but is evolving towards a strategic HR role. Recruitment is certainly competency-based."

Its workforce composition is predominantly (90-95\%) full-time permanent - majority are billable resources. Five to $10 \%$ of its workforce is in enabling functions such as HR, Marketing, and Finance. In back-office operations, temporary workers are employed only for year-end finance and accounting work and for voice operations: they cover Christmas and "New Year specials". Contrary to the extant literature on training access for nonstandard employees (Hall et al, 2000), process-specific training is also provided to temporary workers.

Although certain base qualifications and generic competencies are identified for each role, recruiting teams for a new process give additional consideration to client specifications. Owing to its high and ongoing employee turnover, the organisation specifies two additional selection criteria: career stability and attitude. For its voice processes, communication skills, stability, and attitude are particularly important, while for back-office operations, accounting qualification and 2-3 years' experience are sought. However, the organisation's ability to impose the above selection criteria is somewhat curtailed by the tight external labour market, which has created the need to invest in domain and process-specific training. The Process manager-Voice commented:

\footnotetext{
First we rely on the external recruitment consultants. Second, if one of the big players in the BPO space, like if American Express is doing recruitments, then the supply for Organisation C gets dried up.... The number of people who would turn up would be far less when a big player is recruiting.
}

Given its expansion plans, career planning is widely encouraged. Observation and analysis of various $H R$ documents suggests it has developed a process of internal job 
postings (IJPs), job rotation, and loyalty bonuses for retention. Its performance management system is competency-based and has detailed descriptions of competencies for each role and process. Further, the appraisal process is extremely exhaustive. Some roles undergo monthly performance reviews for each identified competency and its proficiency levels. QAs, in conjunction with the TL, identify regularly the performance gaps and often close them by training interventions.

\section{Analysis of Factors Influencing Provision of Training}

The following section provides an analysis of how various factors affect the nature (externalisation, diversity, and formalisation) and extent (volume) of training. Following Miles and Huberman (1994), a conceptually clustered matrix and explanatory maps were sent to the organisation. The conceptually clustered matrix provides a snapshot of the intensity and direction of the different factors under consideration (see Table 8) and includes notes from the researcher.

\section{Extent of Training}

On average, Organisation $\mathrm{C}$ invests in about 12 days of training per person per annum. The training volume is directly affected by the complexity and size of the process as well as by the temporal dimension of a process's life cycle. For new processes, the training volume is very high, but it tapers off as the process becomes mature. However, the training volume increases again because of persistent and high employee turnover. This pattern was more evident in its voice processes than in its back-office processes. The training volume increases again, for example in its voice processes, whenever a process enhancement or change in process specifications is requested. Organisation C's change in ownership meant that it had to reinvent most of its internal processes to align with its new "captive" business. Training volume also increased with the metric-driven approach adopted by its QMS, which was reflected in its appraisal systems.

\section{Nature of Training}

Its current focus is on technical training, process, domain, and client-specific training. Other training includes learning clients' unique software applications and voice and accent neutralisation. Training needs are identified at different levels. The organisation 
senses and finalises client specifications through SLAs and disseminates them to its operations team. Client specifications are then matched to its current skills pool and fed to HR for hiring specifications. Given its planned expansion, the organisation is now thinking of investing in managerial and leadership skills.

Table 8 .

\section{Conceptually Clustered Matrix of Factors}

\begin{tabular}{|c|c|c|c|c|}
\hline S.No. & Main factors and attributes & Intensity & Respondents & Researcher's notes \\
\hline 1 & $\begin{array}{l}\text { Workplace change } \\
\text {-structural } \\
\text {-ownership } \\
\text {-technological }\end{array}$ & $\begin{array}{l}\mathrm{M} \\
\mathrm{H} \\
\mathrm{H}\end{array}$ & $\begin{array}{l}1,3,4,5 \\
1,3,4,5 \\
1,3,5,7\end{array}$ & $\begin{array}{l}\text { Post-ownership change more changes to structure, technology } \\
\text { and systems to follow. }\end{array}$ \\
\hline 2 & $\begin{array}{l}\text { Market orientation (M0) } \\
\text {-information sensing } \\
\text {-information dissemination } \\
\text {-organisational response }\end{array}$ & $\begin{array}{l}\mathrm{H} \\
\mathrm{H} \\
\mathrm{M}\end{array}$ & $\begin{array}{l}1,2,3,4,5 \\
1,2,4,5 \\
1,2,4,5,6\end{array}$ & On average, it has medium to high levels of M0. \\
\hline 3 & $\begin{array}{l}\text { Learning orientation (L0) } \\
\text {-commitment to learning } \\
\text {-open-mindedness } \\
\text {-shared organisational vision }\end{array}$ & $\begin{array}{l}\mathrm{M} \\
\mathrm{H} \\
\mathrm{M}\end{array}$ & $\begin{array}{l}1,2,4,5 \\
1,3,4,5,6 \\
1,4,5\end{array}$ & $\begin{array}{l}\text { Evidence of critical thinking is present but is moderated by } \\
\text { clients' willingness to accept ideas and any process } \\
\text { improvements. }\end{array}$ \\
\hline 4 & $\begin{array}{l}\text { Quality management systems (QMS) } \\
\text {-Commitment \& sharing information } \\
\text {-continuous improvement } \\
\text {-teamwork }\end{array}$ & $\begin{array}{l}\mathrm{M} \\
\mathrm{H} \\
\mathrm{M}\end{array}$ & $\begin{array}{l}1,3,4,5 \\
1,3,4,5 \\
3,4,5\end{array}$ & $\begin{array}{l}\text { Gradually developing internal and external quality standards. } \\
\text { External quality standards are client-driven. }\end{array}$ \\
\hline 5 & Employee turnover & $\begin{array}{l}\mathrm{H} \& \mathrm{M} \text { to } \\
\mathrm{L}\end{array}$ & $1,3,4,5$ & Process-dependent, e.g. Voice=H; Back Office=M to L \\
\hline 6 & $\begin{array}{l}\text { Enterprise size, complexity and } \\
\text { ownership }\end{array}$ & $\begin{array}{l}\text { M, M,\& } \\
J V \\
\text { (Indian) }\end{array}$ & $1,3,4,5$ & $\begin{array}{l}\text { Medium-sized organisation, small portfolio, limited verticals } \\
\text { and domains. }\end{array}$ \\
\hline 7 & Temporal dimension & $\mathrm{H}$ & $1,2,3,4,5$ & Each new process or enhancement has an impact on training. \\
\hline 8 & Geographical dimension & M & $1,3,4,5$ & Currently, most processes are from the UK. \\
\hline 9 & $\begin{array}{l}\text { Strategic HRM approach and workforce } \\
\text { composition } \\
\text {-rewards and performance management systems } \\
\text {-career planning } \\
\text {-recruitment skills level \& operating roles; and } \\
\text {-use of non-standard employment }\end{array}$ & $\begin{array}{l}\mathrm{H} \\
\mathrm{H} \\
\mathrm{H} \\
\mathrm{L}\end{array}$ & $\begin{array}{l}1,3,4,5 \\
1,3,4,5 \\
1,3,5 \\
1,3,4,5\end{array}$ & $\begin{array}{l}\text { Strong competency-based performance management and } \\
\text { rewards and recognition systems. IJPs and cross-skilling and } \\
\text { career progression evident. Limited use of temporary and part- } \\
\text { time workers. }\end{array}$ \\
\hline
\end{tabular}

Legend: $H=H i g h ; M=$ Medium; $L=L o w ; N=N i l$ 
Operational team leads undergo training for quality standards such as COPC and SS to improve process efficiencies, customer satisfaction, and client relationship management. Training diversity increases when a new process is sourced from a different geographical location or even when a new client is added. Changes to clients' process specifications and the scope of process enhancements demand a new type of training to be imparted. Workplace change to a captive business model is expected to have, in the long run, a negative impact on the volume and diversity of training, because the skills needed for a captive environment are more standardised than those needed for a third-party environment. In a captive model, software applications and standard operating procedures and terminologies standardise the training needs. Moreover, as the experience of a given technology increases, the demand for skilled workers decreases (Bartel \& Lichtenberg, 1987).

Organisation $\mathrm{C}$ has limited reliance on external providers because the bulk of its training is process-specific and internally sourced. External providers were sparingly used for quality certifications, such as SS and COPC, and for developing frontline management and supervision skills.

Owing to its medium to high QMS, a formal approach to training was evident. Its MO capabilities, quality management, and competency-based performance management system facilitate formal identification of training needs. Client specifications require adherence to process metrics; thus, QMS become an objective way of capturing process improvements and formally assessing employees on the training imparted. For most process-specific training, employees have to undergo written tests, and only after they achieve a certain pass mark are they allowed to work on the shopfloor. Likewise, process trainers are required to undergo Train The Trainers programmes and are frequently evaluated.

\section{Discussion}

It follows from the analysis of various data sources that Organisation $\mathrm{C}$ has a medium to high level of MO and a medium level of LO and QMS. In some instances, it has been unable to change its clients' theory-in-use because the perceived benefits of the change relative to clients' needs are low. Its medium LO could also be a reason. Organisation C's 
current LO and MO capability ensures adaptive learning. Its ability to engage in generative learning is limited owing to its current level of LO.

Also evident from the above analysis are the linkages among the enabling roles of its QMS, MO, and LO, and the way the three work synergistically to bring about process efficiencies. Process efficiencies, among other operational benefits, have an impact on the nature and extent of training provided. What is of concern, however, is the narrow focus of its process efficiencies given the nature of quality management frameworks employed at Organisation $\mathrm{C}$.

Organisation C's HRM and training are separate functions. Training is an inextricable part of operations, and the HR department's function in relation to training is one of monitoring training budgets and its logistical arrangements. It is evident from the workforce composition that the majority of its employees are operating staff employed at the lower end of the pyramid. Such a "flattened pyramid" structure implies that the organisation is consciously keeping its employee costs low by gradually developing its training infrastructure to train entry level recruits as well as to deal with ongoing employee turnover issues.

Finally, the analysis points towards the gaps in the Indian educational infrastructure which is ill-equipped to deal with the demand for qualifications and skills necessary for the ITeS/BPO sector.

\section{Conclusion}

Organisation $\mathrm{C}$ needs to strengthen its learning and development infrastructure and QMS to support higher order or generative learning, which is developed through the synergistic effects of strong LO, MO, and quality management capabilities. This is a significant aspect for the organisation to consider, especially if it wants to strengthen its process capabilities and secure long-term cost efficiencies.

If the organisation improves its people management capabilities and process maturity through ongoing investment in training, QMS, and providing career paths, it could reduce its high employee turnover costs, thereby freeing up further resources for higher order learning and capabilities. Such an approach would provide added value and satisfaction 
for clients. Partnerships between industry, academia, and industry associations (for example, NASSCOM) need to be forged so that a range of supervisory training and relevant certifications for the ITeS/BPO sector can be developed. Such certifications would result in the transference of cost of such training from the organisation to potential applicants, thus freeing up organisational resources to build higher order capabilities for a differentiated and value-added service package. 


\title{
CHAPTER 5.4
}

\section{ORGANISATION D}

\begin{abstract}
About Organisation D
Organisation D is a small BPO, set up in 1999 as a green field project by the investment division of a large, diversified Indian firm. Organisation D has two business lines: Medical Transcription (MT) services and Finance and Accounts (F\&A). Initially, it set up a 750-seat MT centre in New Delhi for US-based clients. With high upfront investment, the initial return on investment to its holding company was low. Because MT services has low entry barriers and typifies low-cost mass-production strategies, competition for its services intensified and declining billable dollar rates became the norm. This led to a declining return for the parent organisation.
\end{abstract}

The above situation necessitated a move from a high-fixed-cost model to one with high variable costs. Consequently, it restructured its operations to a sub-contracted vendorbased model. Under this model, all MT work from the US was sub-contracted to about 70 vendors in 12 different cities in India. The restructuring also involved setting up a business development front in the US by acquiring a MT business in the US. Setting up a US front end organisation under a different trade name allowed better market positioning for expanding its MT business portfolio. It acquired US MT companies and transferred their work to Indian operations through its US front end. Under the current business model it has about 550 contractors as part of its vendor-based network for delivering MT services. The organisation employs about 50 permanent employees based at its head office in New Delhi.

To move up the value-chain, in 2002 Organisation D expanded its portfolio to F\&A processes. These cover five sub-accounting processes: accounts payable (AP), accounts receivable (AR), bank reconciliation (BR), financial reporting, and taxation services. Its F\&A business line employs nine employees catering to about 30 clients in three industry sectors: tyre franchisees, mortgage businesses, and CPA houses in the US. Its strategy was to focus on retail and SME sectors in the US. Following the success of the development of a US front end for securing new clients by its MT business model, it replicated the same model for its F\&A business line by acquiring an accounting front end in the US. The main purpose of the front end is to develop business and to act as its 
service delivery channel. In essence, the above business model corresponds most closely to a third-party business model.

\section{Organisation Structure and Processes}

Organisation D's ownership patterns and a high-level structure is shown in Figure 17. For its MT processes, it has established a vendor-based network of MT service providers in 12 cities in India and in two cities in the US. Some cities have multiple centres. Its MT services cover a range of specialist areas, such as cardiology, dentistry, haematology, neurology, immunology, orthopaedics, and urology. It also undertakes special transcription reports, such as an autopsy report, chart notes, clinic notes, discharge summaries, a radiology report, and pathology reports. Its MT team is headed by an operations manager and is supported by a production manager, assistant managers, team leads, and quality analysts (QAs) for each area of medical specialisation. The team leads provide on-the-job training and education support to editors and medical transcriptionists. Editors carry out the first level of quality check, and QAs provide the second level of quality check on the transcriptions.

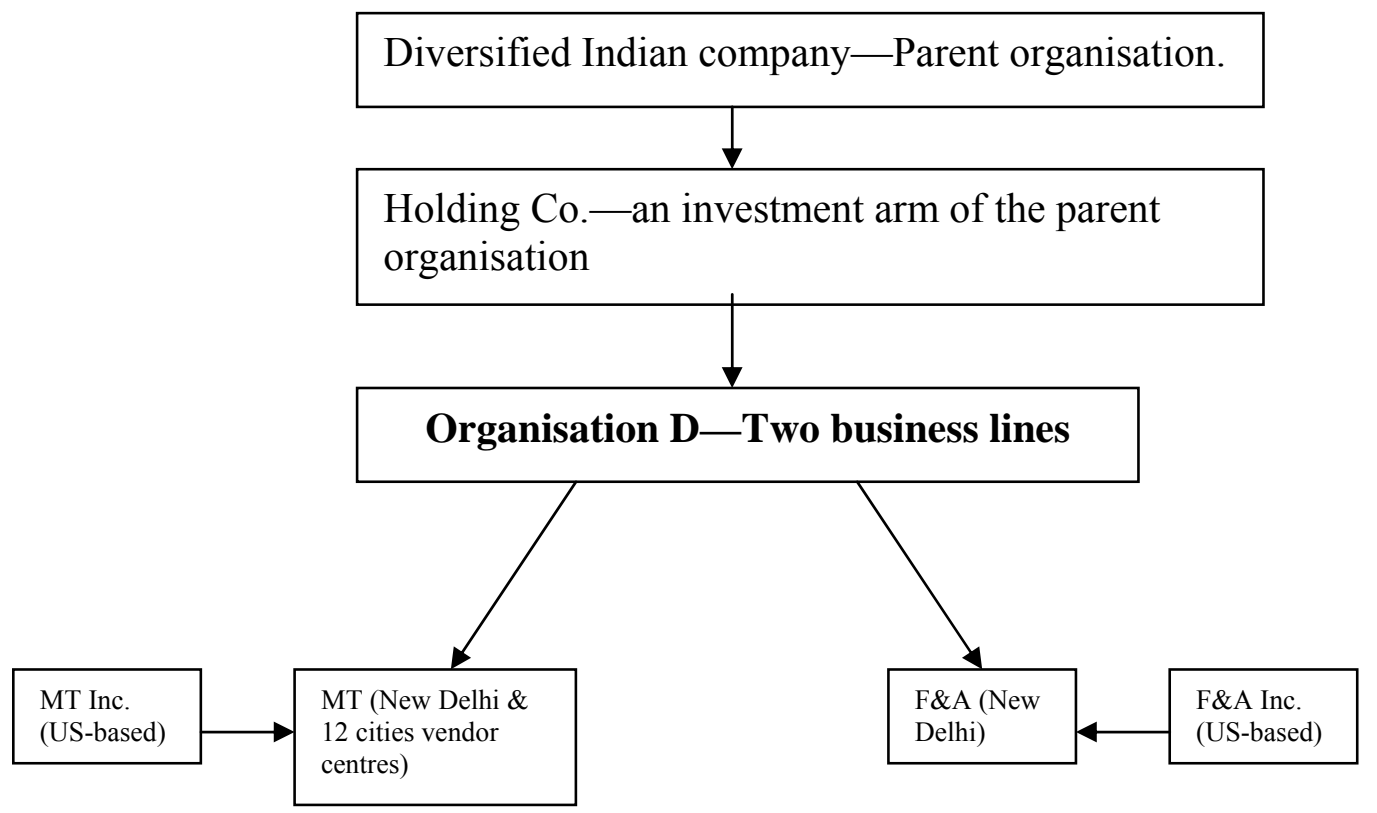

Figure 17. Ownership Structure of Organisation D.

For its F\&A business line, its small and simple team structure is organised around accounting sub-processes: two for AP and AR, three each for BR and finalisation, with one manager overseeing the entire processes. Work received from 30 clients is split equally and by client accounts. Such work organisation allows faster tracking of any errors in the process, and accountability is easier to confirm. 


\section{Business Process, Strategy, and Work Organisation}

Organisation D's business development function is undertaken by its US front ends: MT Inc. and F\&A Inc. Clients do not directly interact with Organisation D; instead, they deal with the US front ends. A typical MT process involves the following steps. The patient sees a medical practitioner or allied staff for some treatment. The medical practitioner and/or the allied staff notes details and dictates them using a hand-held digital Dictaphone or connects to secure dictation servers via 1-800 numbers. By the use of secure transcription servers, data is captured, stored, and encrypted and each audio file is transferred over the Internet for transcription work. These files are first decrypted and then distributed to various vendors for data transcription work. They are then delivered back to MT Inc. via its secure server, and in accordance with HIPPA (Health Insurance Portability and Protection Act) norms and guidelines in the US.

The metrics and SLAs for delivery are negotiated by MT Inc. and communicated to Organisation D to ensure that process delivery adheres to client specifications and AAMT (American Association for Medical Transcription) guidelines. Based on the medical specialisations and strengths of each vendor, work is distributed. Each vendor has a separate service level agreement (SLA) with Organisation D and is supported by a team of QAs and editors employed by Organisation D to ensure a three-tier quality check before transcripts are delivered to MT Inc. Key metrics are included in vendors' SLA cover aspects such as accuracy and error rates and turnaround times.

Given that the billing rates for this BPO service are among the lowest on the continuum of services, a mass-production service market model (Boxall, 2003) requiring minimal investment in skills is logical. Transferring fixed and operating costs to vendors has helped in keeping it afloat. However, it has shortcomings of its own making. For example, it causes limited control over workflow, constraints in offering scalable solutions when big opportunities present themselves, and the issues of managing the differential performance rates of vendors.

The F\&A business line represents a slightly differentiated production system and work is organised as follows. F\&A Inc. receives and scans accounting documents from clients for onward transmission to Organisation D. Next, the F\&A team at Organisation D enters and processes the relevant accounting data in applicable software applications and uploads it 
back via the Internet to F\&A Inc, where further accuracy checks are performed and necessary adjustments made before it is delivered to clients.

\section{Nature and Extent of Training}

On average, employees undergo 4-5 days of new-hire training, and, depending on the process, employees receive 5 (for F\&A) to 10 (for MT) days of training per annum. The nature of training provision is very different for MT and F\&A lines. For instance, in F\&A, the focus is on developing domain knowledge (accounting and finance) of US accounting standards, understanding relevant legislation, and knowing clients' multiple accounting software applications. Being a small organisation, it does not have a separate training infrastructure. The focus is on recruiting experienced personnel with knowledge of US F\&A standards and using their expertise for mentoring and coaching the other team members. Given its skeletal training infrastructure and to minimise training costs for developing capabilities in commonly used accounting application software applications, it has developed self-paced learning resources for six US accounting software applications. These include Peachtree Accounting, Easy Accounting, Quick Books Pro Accounting, and Intuit for Taxation.

F\&A's new hires do not undergo a formal training session upon joining. Most of the training provided (domain-specific, software, and legislation) is informal and on the job, and provided by supervisors via daily mentoring and coaching as and when time and workflow permit. Training imparted here is similar to informal and incidental learning, often noted at small workplaces (Watkins \& Marsick, 1999). Such an approach was easily observable. A collegial and informal environment exists. External training providers are used only for specialist training in US accounting standards, tax legislation and for understanding differences between various US state and federal governments' tax legislation, for example, for filing tax and alimony returns. F\&A has developed self-paced training manuals for reference and study in employees' free time.

The nature of training and the supporting infrastructure are very different for its MT business line. Firstly, the bulk of training needs are transferred to vendors based on their specific expertise and after compliance with HIPPA and AAMT standards and guidelines have been proven. Where certain training gaps exist, knowledge of HIPPA and AAMT is provided through external training providers. Internal training is provided to its team of 
editors, senior proof readers, and QAs to ensure clients' specifications are adhered to and process improvements are made using various quality management tools.

\begin{tabular}{|lllll|}
\hline $\begin{array}{l}\text { New hire } \\
\text { training }\end{array}$ & $\begin{array}{l}\text { Process } \\
\text { \& domain-specific } \\
\text { training }\end{array}$ & $\begin{array}{l}\text { Refresher } \\
\text { training }\end{array}$ & $\begin{array}{l}\text { Team leader } \\
\text { training }\end{array}$ & $\begin{array}{l}\text { Coaching } \\
\text { \& } \\
\text { mentoring }\end{array}$ \\
4-5 days & $\begin{array}{c}\text { 1-2weeks } \\
\text { Informal \& } \\
\text { process-specific }\end{array}$ & $\begin{array}{l}\text { Needs-based } \\
\text { process } \\
\text { enhancements }\end{array}$ & Minimal & High and ongoing \\
2005-2006 key focus areas & & & \\
\hline $\begin{array}{l}\text { Software } \\
\text { applications } \\
\text { knowledge }\end{array}$ & $\begin{array}{l}\text { Domain } \\
\text { knowledge }\end{array}$ & $\begin{array}{l}\text { Process } \\
\text { efficiency }\end{array}$ & $\begin{array}{l}\text { Quality } \\
\text { training }\end{array}$ & $\begin{array}{l}\text { Process-related \& } \\
\text { domain-specific }\end{array}$ \\
\hline
\end{tabular}

Note: Training is mostly informal for F\&A and highly structured and formal for MT business line.

Figure 18. Temporal dimension of training: Changing emphases.

All transcriptionists and editors undergo rigorous testing and an interview followed by a basic training module. They do not commence production until they have reached a level of $98 \%$ medical correctness in their transcriptions. Focused vendor and employee recruitment ensures that the right levels of skills and experience in a given field of speciality is present. In essence, the training for MT business is much more formal and structured and follows an evaluation process. Both business lines do not support any education or soft-skills training.

\section{Analysis of Factors Influencing Provision of Training}

\section{Workplace Change}

Following the restructuring of its MT business line, the focus of its training infrastructure has changed significantly. Its overall volume of training has declined with the introduction of a vendor-based structure. However, its training diversity has increased for its group of QAs, editors and proof readers. Further, owing to the complexity of the logistics of managing a vendor-based model, new modules for relationship management and training for reporting protocols and monitoring of metrics have been created. The adoption of multiple accounting software applications for its F\&A business line resulted in additional, albeit limited, learning needs, as the learning is delivered informally. 


\section{Quality Management Systems (QMS)}

Organisation D's quality systems are primarily focusing on meeting and in some cases, exceeding the clients' specifications. It utilises various quality management tools to ensure service metrics as per SLAs are delivered. The MT business line has a QA centre that ensures that every single deliverable is certified as a quality deliverable. Its three-tier quality check of editors, proof-readers and QAs enables such delivery. Six Sigma (SS) methodology is used to measure the performance of its vendors and employees. As such, there is a need for its editors, account managers, and QA team to learn various SS, compliance, and other quality management tools. Its ability to implement continuous process improvement is limited, because some clients may not want to bring about process improvements. Overall, it has a fairly set quality management agenda, as noted in one of its quality documents:

\footnotetext{
In Medical Transcription domain our QA procedure makes a commitment to our clients that it will provide transcriptions which will have accuracy level of $98 \%$ or more. We at Organisation $\mathrm{D}$ achieve this level of accuracy by using a 7-point measure given below:

Reports have all essential components and all required and relevant information; reports are consistent both within themselves and externally with the other parts of the patient record, should they are made available to Organisation D; discrepancies are flagged for the dictator; reports have the proper structure, format, content, spelling and grammar; reports are transcribed to accurately reflect the meaning intended by the dictator, adhering to AAMT norms and guidelines, unless client specifications dictate otherwise, e.g., verbatim transcription; the patient's right to confidentiality is strictly guarded; and reports are timely in their completion and submission.
}

Its internal quality management capabilities are developed in a narrow range, which, together with its limited LO ability, restricts the organisation to develop higher order process maturity. To develop its quality management capability, it recruits people externally with experience in specific quality management tools.

\section{Market Orientation}

Organisation D's MO ability is geographically distributed between the US and India. Client specifications and SLAs are negotiated by its US business fronts. MO ability for Indian operations is not strong as there is limited direct interaction and integration 
between the US and Indian operations. The brief for its US counterpart is specific. Given the mix of mass service and slightly differentiated service strategy (Boxall, 2003) it follows, business opportunities are explored and secured in a narrow range. The above strategy does not preclude it from exploring value-added services within its existing specialisations, for example, the implementation of SOX (Sarbanes-Oxley) legislation compliance for its own business in the US and gradual undertaking of additional SOX processes with existing clients.

Overall, owing to its relatively simple portfolio of business processes, disseminating client specifications to delivery teams in India is easy. Responses are framed initially by pre-testing and a pilot run, and finally when the process goes live. Training requirements for its US business front is not Organisation D's responsibility. Organisation D provides training to its operations team in India, who are responsible for disseminating client information and framing appropriate operational response.

\section{Learning Orientation}

There is evidence of challenging clients' specifications for both its MT and F\&A lines (albeit limited). Open-mindedness is evident in process improvements after the process goes live. Process Manager-F\&A noted:

\footnotetext{
Earlier, what we were doing is we were punching the data in the system. But one of the team members from this team, from Accounts Payable team, has designed a macro in Excel, in which actually you ask when the data has been received from a client in Excel format ... . Now, what they are doing is, they have designed a template which can be uploaded into the software. So, earlier it was a six hour job, but now it is a six minute job."
}

The extent to which such improvements impact on training depends on who initiates the process improvement request. If it is initiated from a team member the training impact is minimal as the solution was generated from within the team. However, if the process improvement is requested by the client, it results in refresher training. Training is also triggered by the feedback received from clients on the service provided. Because of the commoditised nature of its services and the lack of direct interface with clients, its low LO and medium MO abilities can only ensure a reactive approach to the sensing, disseminating, and framing of training and operational responses. 
A strong (albeit informal) communications channel has enabled the development of a shared vision. There are few formal meetings between and across teams. A strong, handson and on-the-job approach to problem solving and communicating change was observable during the visit. The organisation's simple business lines and a vendor-based model do not require it to expand its commitment to learning. The focus is on providing essential training, most of which is client and role-specific.

\section{Employee Turnover}

Given its vendor-based model for MT service, employee turnover is not a concern for the organisation. Even for its F\&A service line, employee turnover is between 12 and 15\% per annum, which translates to about one resignation per annum. This is significantly lower than the industry average of about $30-40 \%$. As a result its impact on the nature and extent of training provided is minimal. One explanation provided by the F\&A manager was:

\footnotetext{
Because, see, there are hardly nine people. It's very easy to monitor nine people, and you can very easily understand their training needs, and subsequently then address it. The moment you grow in the ladder, the moment you say you are having 250 or 300 people, more control is required and there are more chances that the people might slip. So, that is the reason why other companies are having turnover ratio and employee turnover as an issue, while ours are very small.
}

Despite low attrition levels, the above respondent noted that employee attrition is desirable, especially for bringing about process improvements-especially at lower levels. At agent and supervisory levels, focused recruitment and selection could see the adoption of established good practices from larger organisations, whereas employee attrition at senior management levels is desirable for the expansion of its business portfolio (Banerjee, 2004). In both cases, employee turnover is likely to result in additional training and learning.

\section{Enterprise Size, Complexity, and Ownership}

Its small size and a relatively simple portfolio of processes (low process complexity) is one of the reasons for its low training diversity and volume. Because it is a subsidiary of a large diversified Indian organisation, there is pressure from its holding company to 
maximise its return on investment. To this end, it invests only in process-specific and project critical training or, where return on investment is high. Consequently, its HR practices have a cost-minimisation agenda. It attracts people with relevant skills and domain-specific experience to contain its training infrastructure costs.

\section{Temporal Dimension of Processes}

Its focused recruitment and selection strategy, low employee attrition, and a vendorbased model significantly reduces the impact that temporal dimension of processes have on training. Although its MT line receives process enhancement requests from clients to modify the SLAs, its existing capabilities and vendor-based model do not allow all enhancement requests to be serviced; consequently, it has a minimal impact on training provision. Further, as the organisation has been in the MT business line since 2000, considerable experience of the processes, and low employee turnover have limited impact on training provision (Bartel \& Lichtenberg, 1987).

\section{Geographical Dimension}

As all its clients are based in the US, the geographical dimension has a limited impact on provision of training. Only a few F\&A processes are affected; for example, while filing alimony returns for clients, agents need to know different US state and federal legislation, as they vary significantly. Differences in law are communicated by team leads to its members by ongoing coaching and mentoring. Despite the relatively limited impact of the geographical dimension on training in Organisation D, it was noted as an important factor by the Manager of F\&A:

\footnotetext{
Yes, that is different, because if you are moving from US to UK . . .I'll give you a very small reason. In the US, the inventory is valued at a market price; in the UK the inventory is valued at a cost to market price or whichever is less. So there is a lending requirement for UK . . . so now UK is having a different set of valuation of inventory, US are having different set of valuation of inventory. So we need to be trained on all if you are moving from one geographical location to another geographical location.
}

For its MT business line, although medical terminologies are universal in nature, some training is needed to adhere to country-specific legislation such as HIPPA, and AAMT guidelines. 


\section{Strategic HRM Approach, Skills Level, and Workforce Composition}

Although there is evidence of a strategic approach to recruitment and selection, Organisation D's training function is totally devolved to line managers. For other HR practices, HR has administrative and fire-fighting roles. As noted by their F\&A manager: "HR plays a role in addressing the grievances of employees, but not on training needs . . . because all the training is done on the production floor."

The strategic recruitment and selection strategy to some extent reduces the need for process and domain-specific training. Organisation D follows different performance management systems for both business lines. For F\&A employees, it follows 360-degree feedback and uses it as a developmental tool. As the opportunities for career development in F\&A are fairly limited, managers identify opportunities for cross-skilling and job rotation to retain staff and reduce process monotony. For its MT service, employees follow a combination of rating and competency-based systems. Through its performance management system and inputs from QAs, performance and competency gaps are identified and, where appropriate, training is provided. The nature of the organisation's workforce is full-time permanent for both MT and F\&A groups, except for its vendor network, which can be regarded as a non-standard workforce. The vendor structure has reduced its direct investment in training, although it is difficult to gauge the nature and extent of training provided by its vendors. It follows from the analysis that it is the experience of employees on a given process, low employee turnover, and the relatively simple and stable nature of processes that creates a limited demand for training.

\section{Impact of Factors on the Nature and Extent of Training}

The following section provides an analysis of how various factors impact on the nature (externalisation, diversity, transferability, and formalisation) and extent (volume) of training. Miles and Huberman (1994) suggest, a conceptually clustered matrix is the first step to develop explanatory networks, as it provides a snapshot of the intensity and direction of the different factors under consideration with notes from the researcher (see Table 9).

\section{Extent of Training}

Organisation D invests between 5 and 10 days of training per person per annum. Nature of training focuses on developing competencies in quality systems (see Figure 18) such as 
HIPPA and AAMT standards and guidelines. F\&A's ongoing training needs are met by informal coaching and mentoring. Such learning includes changes to US accounting standards and state and federal government's tax legislation, and upgrades to the functionality of six accounting software application packages in use. Training volumes

Table 9.

A Conceptually Clustered Matrix of Factors

\begin{tabular}{|c|c|c|c|c|}
\hline S.No. & Main factors and attributes & Intensity & Respondents & Researcher's notes \\
\hline 1 & $\begin{array}{l}\text { Workplace change } \\
\text {-structural } \\
\text {-ownership } \\
\text {-technological }\end{array}$ & $\begin{array}{l}\mathrm{H} \\
\mathrm{L} \\
\mathrm{M}\end{array}$ & $\begin{array}{l}1,2 \\
1,2 \\
1,3,4,5\end{array}$ & $\begin{array}{l}\text { High structural changes-vendor-based model, } \\
\text { multiple accounting software applications }\end{array}$ \\
\hline 2 & $\begin{array}{l}\text { Market orientation (M0) } \\
\text {-information sensing } \\
\text {-information dissemination } \\
\text {-organisational response }\end{array}$ & $\begin{array}{l}\mathrm{M}-\mathrm{H} \\
\mathrm{M} \\
\mathrm{M}\end{array}$ & $\begin{array}{l}1,2,3 \\
1,3,4 \\
1,3,4\end{array}$ & $\begin{array}{l}\text { Medium level of M0. Geographically distributed } \\
\text { between US and India. }\end{array}$ \\
\hline 3 & $\begin{array}{l}\text { Learning orientation (L0) } \\
\text {-commitment to learning } \\
\text {-open-mindedness } \\
\text {-shared organisational vision }\end{array}$ & $\begin{array}{l}\text { M-L } \\
\text { M-L } \\
\text { M-L }\end{array}$ & $\begin{array}{l}1,3,4 \\
1,3,4 \\
1,3,4\end{array}$ & $\begin{array}{l}\text { Limited evidence of critical thinking is present. It is } \\
\text { Affected by clients' willingness to accept process } \\
\text { improvements. }\end{array}$ \\
\hline 4 & $\begin{array}{l}\text { Quality management systems (QMS) } \\
\text { - Commitment \& sharing information } \\
\text {-continuous improvement } \\
\text {-teamwork }\end{array}$ & $\begin{array}{l}\mathrm{M} \\
\mathrm{M} \\
\mathrm{H}\end{array}$ & $\begin{array}{l}1,3,4 \\
1,3,4 \\
3,4,5\end{array}$ & $\begin{array}{l}\text { Gradually developing internal quality management } \\
\text { competencies. Limited application of standards due to nature } \\
\text { of work and clients' unwillingness to seek higher value add; } \\
\text { some metrics are still client-driven. }\end{array}$ \\
\hline 5 & Employee turnover & $\mathrm{L}$ & $1,3,4,5$ & $\begin{array}{l}\text { Low attrition. Hard to compute turnover for its } \\
\text { vendor-based model. }\end{array}$ \\
\hline 6 & $\begin{array}{l}\text { Enterprise size, complexity and } \\
\text { ownership }\end{array}$ & $\begin{array}{l}\text { L, M-L \& } \\
\text { Indian }\end{array}$ & $1,3,5$ & $\begin{array}{l}\text { Small-sized organisation; small portfolio, limited } \\
\text { verticals and domains. }\end{array}$ \\
\hline 7 & Temporal dimension & $\mathrm{L}$ & $1,3,5$ & Process enhancements have limited impact on training. \\
\hline 8 & Geographical dimension & $M-L$ & $1,2,4,5$ & Currently, most processes are from the US. \\
\hline 9 & $\begin{array}{l}\text { Strategic HRM approach and workforce } \\
\text { composition } \\
\text {-rewards \& performance management systems } \\
\text {-career planning } \\
\text {-recruitment skills level \& operating roles; and } \\
\text {-use of non-standard employment }\end{array}$ & $\begin{array}{l}\mathrm{M}-\mathrm{H} \\
\mathrm{N} \\
\mathrm{H} \\
\mathrm{L}\end{array}$ & $\begin{array}{l}1,3,4,5 \\
1,3,5 \\
1,3,5 \\
1,3,4,5\end{array}$ & $\begin{array}{l}\text { Competency-based and 360-degree feedback. } \\
\text { Rewards and recognition for process improvements. } \\
\text { Cross-skilling and job rotation evident. } \\
\text { No use of temporary and part-time workers } \\
\text { for F\&A. Significant outsourcing for MT services. }\end{array}$ \\
\hline
\end{tabular}

Legend: $H=$ High; $M=$ Medium; $L=L o w ; N=N i l$ 
increase when there is a significant change in client specifications, for example, when a client requests an end-to-end process automation.

\section{Nature of Training}

The nature of training provided is technical and job-specific and there is no support for soft skills or higher education. Demand for soft skills is less because of its medium MO capability of the Indian operation and the limited client-facing roles it has to support. Following the restructuring of MT services and the consequent reduction in enterprise size, the need to invest in supervisory, people management, and leadership skills has also declined. Process-specific training is provided internally. Only for specialist tax and accounting legislation modules does the organisation engage the expertise of few external training providers.

Formalisation of training is high for its MT business line, owing primarily to the nature of the process and work organisation (see Figure 17): the structure requires constant evaluation and checking of work by its vendors. Formalisation of training is further strengthened by the use of competency-based performance management systems and by clients' requirement that employees be certified as conversant with relevant MT standards before they work on them. Thus, the organisation has to provide training on a range of quality management tools for developing its QAs and editors. Training is informal for its F\&A business line, partly because of its small team size and partly because of a general resource constraint that small companies face. Learning is achieved in an informal and incidental manner.

\section{Discussion}

It is evident from the above analysis that its simple and stabilised portfolio of processes, low employee turnover and, earlier structural changes have resulted in an overall decline in training needs (Bartel \& Lichtenberg, 1987). What also follows from the above analysis is that MO is not a localised concept - it is geographically distributed - and is affected by the strength of a firm's LO and QMS capabilities. Given the costminimisation strategy evident in the organisation's production function, its commitment to learning is fairly limited, resulting in low LO. Similarly, its quality management systems are targeted to deliver only minimum and mutually agreed client-specific process efficiencies. The approach is reactive and is also reflected in its inability to challenge 
critically clients' assumptions. Consequently, the interaction between its MO, LO, and its QMS yields nominal results and only modelling or adaptive learning is happening (Baker \& Sinkula, 2002). Thus, the organisation's ability to enhance the organisation's overall process capabilities and maturity is limited.

It follows from the analysis that one of the basic tenets of human capital theory — of firms investing in job-specific and technical training rather than generic and behavioural training - is applicable, especially to small organisations that have a relatively simple and stable portfolio of services. Strategic recruitment and selection practices have also minimised the need for training through focused recruitment of experienced employees. Performance management systems, geographical dimension, extent of process enhancements, and client specifications variously affect the nature and extent of training. Only when a new process is added to its portfolio or a major process enhancement is requested does training need arise. Organisation D's training responsibility does not rest within its HR infrastructure; it is devolved to line managers, who focus on imparting technical and process-specific training.

\section{Conclusion}

Although NASSCOM - the industry's association - has established a national centre for the assessment of competence, there are still considerable gaps in the wider Indian educational system. Programmes are lacking in developing industry and domain knowledge, as well as in the understanding of relevant international legislation affecting the industry and its domains. Inclusion of modules that address these gaps in its curriculum by educational institutions or NASSCOM-sponsored certifications would go a long way in supporting small and medium-sized enterprises such as Organisation D. Moving up the value chain would require building higher order organisational capabilities such as project management, the client-specific capabilities, LO and QMS. 


\title{
CHAPTER 5.5
}

\section{ORGANISATION E}

\begin{abstract}
About Organisation E
Organisation E is an extremely large, diverse, and complex offshore development facility of a US-based ITSS multinational. Organisation E provides end-to-end information technology solutions (hardware, software, consulting, infrastructure management, and ITeS/BPO) for its US parent organisation from five locations in India. At the time of the researcher's visit, Organisation E employed about 35,000 people across the following business divisions:
\end{abstract}

- application management services (about 16,000)

- global services delivery centre for ITeS/BPO services (about 10,000)

- business transformation organisation (about 3000)

- software and research development labs (about 3000)

- business consulting services (about 3000).

The organisation provides its services to a range of industry sectors, such as automobile, financial, chemical and petroleum, telecom and media, and business consulting. Organisation E focuses on global IT and software development competencies. The focus of this case study is on its application management services (AMS) division.

\section{Organisation Structure and Processes}

Organisation E's business lines are structured around technology competencies. Its AMS division provides services in the following competency areas: mainframe, enterprise application development, Oracle, PeopleSoft/Baan, Siebel, SAP, RDBMS, data warehousing and business intelligence, enterprise application interface, testing, portal and content engineering, Lotus Notes, Unix, $\mathrm{C}$ and $\mathrm{C}++$, and supply chain management (CAD/CAM).

Ninety-two per cent of its 16,000 employees working at AMS division are deployed on various technical competencies and 3\% work in enabling and business services functions 
such as HR, finance, business operations, quality management, and centre for competency development. The remaining 5\% (about 800) are on bench time (bench time is a term used to describe people who are waiting to be deployed on projects. Till such time, they usually engage in developing their skills in various competencies.)

\section{Business Processes, Strategy, and Work Organisation}

The majority of the employees are working in technical roles in the competency areas noted above. The workforce composition of its AMS division includes close to $84 \%$ employed at entry level (Band 6) — called techies - and their work experience ranges from 0 to 5 years. About $12 \%$ are in Band 7 having 5-15 years of work experience. The remaining 4\% are in Bands 8 and 9 and have work experience ranging from 10 to 20 years or more. Further, nearly a third of all Band 6 employees have less than 18 months of work experience. This composition gives an indication of its people management strategy-low cost and relying increasingly on entry level graduates. A typical hierarchy of roles is as follows: graduate engineer trainees, software engineers, developers, lead developers, team leads, project managers, programme managers, delivery head, managers, solution leads, industry experts, and business analysts.

Over the last five years, the organisation has expanded its business lines and changed the workforce composition significantly. Initially, nearly $90 \%$ of its workforce comprised highly experienced and skilled employees. Now about $60 \%$ are highly skilled and experienced and the remaining $40 \%$ are in the $0-4$ years work experience range. The exact nature of the workforce composition varies for each business line. For example, in business consulting services, the majority (80-85\%) of its employees have about 8-12 years of work experience.

In most offshoring decisions, among other decision criteria applied, a significant criterion is the cost of skills arbitrage between the US and India. The rationale for Organisation E's AMS division is similar and is based on the difference in economic contribution every billable employee can make. On careful analysis, it follows that the difference in contribution per employee reduces as one goes up the organisational hierarchy because the cost of experienced human resources increases with every additional unit year of experience. For example, on average, the industry allows about 110,000 Indian rupees (Rs.) (or US\$2750) for each year of experience. This cost is in addition to an average 
base salary of about Rs. 400,000 (US\$10,000) per annum for graduate engineer trainees (see for example, www.freshersworld.com/forums). Thus, it makes sense for large organisations such as Organisation E to have a large percentage of their workforce at lower levels with limited experience and a base (bachelor's degree) engineering qualification.

The above business and HR strategy creates a different set of problems: that of developing the capabilities of its largest work group - people with 0-4 years of work experience. Thus, it becomes imperative to develop a learning and development infrastructure, if the organisation wants to build technical and managerial competencies, and to continue to increase the ratio of freshers to experienced employees in its workforce composition.

\section{Nature and Extent of Training}

Organisation E has an elaborate learning and development programme for technical, management, and leadership competencies. Organisation E's parent organisation has a well developed online Global Campus corporate university, offering a vast range of training and education programmes covering technical, behavioural and management development competency areas. Global Campus resembles a blend of Taylor and Patton's (2002) "polymorphous" and "chateau" type corporate university and Walton's (1999) "third-generation" corporate university. Such an approach to learning and development represents a resource-based view of the firm aimed at developing sustained competitive advantage through internal investment in human resource development capabilities (Barney, 1991).

Training is mandated at two levels: for new hires and existing employees. For all existing employees, it is 10 days per person per annum and focused on developing project management skills and technical competencies, and in fulfilling specific career development needs of its employees. Training provision for new hires varies from 8-10 weeks depending on the business line and competency areas. Orientation training of 2 weeks is followed by 4-6 weeks of competency-specific training, which includes some soft skills and management training. Once the above training is completed, new hires undergo 2-3 weeks of project-specific training. Such training may include clientprovided training. The focus of client-provided training is initially to develop 
familiarisation with clients' proprietary applications and tools and then to develop proficiency in using these tools. Client-specific training is in addition to the organisation's mandated training.

In terms of the nature and diversity of training arrangements, the bulk of training (85$90 \%$ ) focuses on technical competencies, as noted above. The remaining 10-15\% focuses on behavioural, soft skills and management and leadership development competencies. The training design and delivery utilises a combination of structured and unstructured learning strategies. While the former mode of delivery is common for freshers, the latter relates more to project team members and usually has strong on-the-job training and a "learning by doing" focus.

Identification of training needs follows a structured process prescribed by its quality management capability development model. Despite a large employee base, training is mostly provided by external training providers. Organisation $\mathrm{E}$ has a rigorous process for selecting training vendors, wherein detailed performance metrics are developed for evaluating the external training providers' performance. Evaluation of training, although rigorously undertaken, comes close to Kirkpatrick's first two levels of training evaluation (Kirkpatrick, 1998).

In the US, online and computer-based training is a cost-effective option. Even though the US parent would like all employees in India to undertake the 10 days per annum mandated training through its Global Campus web portal, it is not a desirable or practicable option for the learners in the Indian cultural context. Learners at Organisation E do not feel comfortable with the independent, self-paced, and self-directed learning mode. Classroom-based or facilitated training is typical and suited to the Indian cultural settings for all types of learning initiative.

One of the reasons for the low uptake of Global Campus in India is the high US dollar cost per course per person charged by its US parent. Therefore, to overcome resource constraints, Organisation E has an arrangement to use the content from Global Campus courses and deliver it through its pool of external training providers. Another reason for externalisation of training is the high cost of real estate and technology infrastructure in India. The geopolitical and economic climate in the US determines the nature and extent of exposure to offshoring projects and fixed costs commitments in India. The break-even 
point to recoup such high fixed costs is much longer and higher. Figure 19 provides a snapshot of the changing emphasis of training provided. The competency development centres (technical and managerial) receive annual competency forecasts from various competency leaders across all business lines, based on which the nature and extent of training are determined.

\begin{tabular}{|c|c|c|c|c|}
\hline $\begin{array}{l}\text { New hire } \\
\text { training }\end{array}$ & $\begin{array}{l}\text { Technology } \\
\text { competency } \\
\text { training }\end{array}$ & $\begin{array}{l}\text { Project } \\
\text { training }\end{array}$ & $\begin{array}{l}\text { Management } \\
\text { \& leadership } \\
\text { training }\end{array}$ & $\begin{array}{l}\text { Client } \\
\text { funded } \\
\text { training }\end{array}$ \\
\hline 1-2 weeks & 4-6 weeks & $\begin{array}{l}2-3 \text { weeks } \\
\text { Project/product } \\
\text { dependent }\end{array}$ & $\begin{array}{l}\text { Role-dependent } \\
\text { \& ongoing }\end{array}$ & $\begin{array}{l}\text { Project } \\
\text { dependent }\end{array}$ \\
\hline \multicolumn{5}{|c|}{$\begin{array}{l}\text { (TIMELINE DEPENDENT ON THE NATURE OF PROJECT AND } \\
\text { TECHNOLOGY COMPETENCIES) } \\
\text { 2005-2006: key focus areas }\end{array}$} \\
\hline $\begin{array}{l}\text { New hire } \\
\text { orientation }\end{array}$ & $\begin{array}{l}\text { Competency } \\
\text { training }\end{array}$ & $\begin{array}{l}\text { Project \& client } \\
\text { knowledge }\end{array}$ & $\begin{array}{l}\text { Mandated \& } \\
\text { role-specific } \\
\text { competencies, } \\
\text { \& domain expertise }\end{array}$ & $\begin{array}{l}\text { Proprietary } \\
\text { client tools \& } \\
\text { applications } \\
\text { e training }\end{array}$ \\
\hline
\end{tabular}

Figure 19. Temporal dimension of training: Changing emphases.

\section{Analysis of Factors Influencing Provision of Training}

\section{Workplace Change}

Since its inception, Organisation E has undergone numerous workplace changes. The most significant changes from a training and development perspective include:

- a different workforce composition model, comprising an increasing number of graduate engineer trainees

- greater automation and standardisation of software development processes using capability maturity models and quality management frameworks

- the creation of new and diverse roles and competencies due to increasing business complexity.

Recruiting an increasing number of freshers has had a significant impact on training needs. This change required development of strong learning and development capabilities in a range of technology and management competencies, not only to cope with the 
deficiencies in the undergraduate engineering curriculum, but also to develop in-house capabilities for meeting new technology skill demands.

Owing to the large scale and long-term nature of contracts in its software development projects portfolio, the use of "best-practice approaches" and "standardised protocols" for software development is logical. Organisation E is a CMMI-Level 5 accredited organisation, and utilises the above quality management framework to increase the organisation's process maturity and service predictability. QMS also improve its project management, $\mathrm{MO}$ and $\mathrm{HR}$ capabilities, thus reducing costs and increasing customer satisfaction.

The high rate of technological change and consequent skills obsolescence is a significant driver for investing in new skills. Skills development aids in employee retention and prepares the organisation for exploiting opportunities in new technology areas. Finally, increasing business lines and service complexity has resulted in the creation of new roles and the development of new role-based competencies.

\section{Quality Management Systems (QMS)}

Organisation E is CMMI Level-5 and ISO-certified and follows numerous other quality tools. All its software development processes are mapped against the CMMI framework. In addition to CMMI and ISO, Organisation $\mathrm{E}$ has its own internal quality standards for a number of competency areas, which were developed long before it applied for CMMI and ISO accreditations. For example, according to its HR manager, an "Organisation Ecertified project manager" commands higher market value than a person from the Project Management Institute (PMI) in the US.

Organisation E's QMS focus on infusing process discipline and rigour at various stages of the software development life cycle. There are mandatory quality check points at each stage of the process. Its QMS create an awareness of the role of quality, emphasise client orientation and process compliance, and ensure project deliverables are met. Thus, quality management has a two-pronged focus-process compliance and evaluation, which is applied internally and externally. The project and business development managers estimate project specifications based on internal project experience and performance measures. 
To understand the emergence of QMS in software development, it is critical to provide some historical context. One of the main problems in the early stages of the software development life cycle was the lack of detailed documentation of how the codes were developed. The introduction of quality management frameworks enabled the documentation of critical processes in software engineering, which now has standards for writing codes. An HR manager vividly summarised this change:

\begin{abstract}
What were the thought processes of a programmer when he was creating the code? One of the biggest myths of software engineering was broken when they brought in quality measures. And they said, 'OK, everything has to be documented'. With that came out a lot of aspects of software engineering, which says: what are the standards with which you write a program? You don't just write a programme with whatever just comes to your mind. There are formal standards, accepted standards, with which you work. The nomenclature is in a particular fashion. You cannot write or code in a different fashion than what has been set as a standard or norm.... There are frameworks. Then, you have to measure against these frameworks - that's what it was all about. So, if you go back into productivity improvement or anything, in any context, be it JIT, or any other process, the focus was to extensively narrate what you are doing and why you are doing it. How have you arrived at a particular decision for a process?
\end{abstract}

The specific focus is on minimising the variation from pre-established "best practices" and, consequently, reduce costs, defects and variation, and increase customer satisfaction. To this end, each project is aligned with one or two quality management resources, who oversee the implementation of quality standards and processes. A project manager explains:

They [quality management people] have a specific requirement at every new stage. They check the project. So ... ok, where are we? What is left? We need to follow evenly quality methodology for every project, so that is being done from a quality team. Everything, I mean, [for] every project or every competency we have a process in place and ... they have an appraisal methodology like a flowchart. We have an internal audit team coming from the quality system. They are in place just to ensure whatever we have put together.... Are [we] following it or not?

Thus, the application of QMS in software development impacts on training at different levels. First, understand various documented quality standards for each process. Second, learn various quality management tools and techniques for implementing any process improvements in software development. Third, provide training to its specialist and 
centralised pool of quality management resources to ensure they can implement and apply various quality standards across the organisation, at various stages of the software development life cycle. Finally, such standards influence formalisation of training needs identification, delivery, and evaluation.

Over time, the organisation has documented quality management standards in software and has models for accurate estimation of project time and effort. The use of such quality management processes has also increased the organisation's ability to simplify and standardise project tasks right from business requirement specifications stage. Using internal cost and effort estimation benchmarks, its solutioning team senses information that is critical to project success and proposes solutions that are doable within the project specifications. The structuring and standardisation of the software development processes has simplified the nature of work, enabling the organisation to develop the less skilled and less experienced employees (new hires) by providing fundamental competency-based training for them in a narrow range.

\section{Market Orientation}

Organisation E has a geographically distributed software applications development process. As a captive offshore facility, Organisation E's business development, solutioning, and consulting for higher level design is undertaken by its parent organisation. As such, information sensing and challenging clients' assumptions happen in the US at the solution design and architecture development stage. Once the solutions are specified and agreed upon, the project management team in India takes over the responsibility for disseminating the project information and framing operational responses using established quality management "best practices". Training responses are developed by its Centre for Competency Development. Overall, Organisation E's MO ability is medium to high.

The use of standard project management tools, client relationship management systems, and quality management process requirements enables the capturing of project-specific information in a precise manner. Use of standard documented quality management processes allows project team members to propose and develop applications in a very structured manner. Over time, Organisation E has developed strong quality management and technical capabilities. Consequently, its MO ability is likely to develop further as its 
ability to engage in solutions and the architecture design stage of the software development lifecycle increases.

\section{Learning Orientation}

Organisation E has a strong commitment to learning, which is evident in the extensive provision of training. It has numerous technical competency-sharing networks-Share Nets - across the organisation. Each competency leader is expected each year to contribute towards their Share Nets. Further, employees are encouraged to submit practitioner and research papers in their competency areas for sharing and for evaluation by internal experts. A team of experts review such outputs and publishes the top 10-15 papers each year.

There is evidence of critical thinking and challenging the status quo to bring about process improvements. For example, Organisation E convinced the US office to reduce the hiring specifications of 10 -years work experience for engineers to 5 years. However, the strong levels of open-mindedness do not mean that employees are allowed significant latitude in carrying out their tasks in an innovative manner. There is limited scope for creativity at lower levels as most projects have specific sets of tasks, which are signed off by the clients and follows a "best practice" process for its development work. The nature of work undertaken by developers is in a narrow range. An HR manager noted, "You don't do it where your inflection point is steep.... At the end of the day, it is a series of handed-down solutions to be developed."

It is important to note that process improvements are structurally embedded in its QMS and have a direct impact on training. One project manager observed, "The entire structure of CMMI focuses on process problems. Any process improvement areas are discussed. With proper training, a taskforce is set up for improvement." Overall, Organisation E has medium to high LO. Its QMS enables it to challenge the status quo, but to a limited extent using its structured processes.

\section{Employee Turnover}

Organisation E currently has an employee turnover rate of $16 \%$ per annum. This attrition rate is significant because, on average, it results in recruiting about 5500 employees per annum in different competency areas and at different job levels. Recruitment arising from 
employee attrition and business expansion needs results in recruiting between 12,000 and 14,000 employees each year. Such extensive employee turnover and recruitment puts ongoing pressure on the volume of training.

Employees usually leave for reasons such as better career opportunities (monetary reasons and higher responsibility) and their ability to continuously up skill by working on new technology projects. Employee turnover is highest in the 3-6 years of work experience group because the external labour market offers a wage premium for employees with 5 plus years of experience at an MNC. Thus, investment in training in new technology is critical for employee retention.

\section{Enterprise Size, Complexity, and Ownership}

An increase in the number of employees and in high project complexity affects training provision. While the volume of training has increased as a result of the greater number of employees to be trained, training diversity has increased due to the gradual addition of new business lines, IT competencies, introduction of new business processes, and implementing QMS. The exact nature and extent of training varies in each business group. Organisation E demonstrates strong ethnocentric approach to its business and HR practices. The parent organisation's strong global brand image attracts new hires because they are aware of its commitment to the learning and development of its employees.

Its move to the new business model of having a higher proportion of graduates is a point of difference with its global operations. This change not only reflects the need to expand its presence in India, but is also an emulation of the dominant business model adopted by most large Indian IT players, such as Infosys, Wipro and Tata Consultancy Services. There is still a difference in the modus operandi of this model at Organisation E. In terms of the provision of training, there is a heavy reliance on external training providers rather than the dedicated in-house learning and development facility typical of large Indian ITSS players.

\section{Temporal Dimension of Processes}

While the above discussion provides understanding of some macro-level factors influencing provision of training, it is critical to understand the variation in training needs at various stages of a project's life cycle. Given that work is undertaken in established 
global technology competencies, there is a minimal impact on training from projects from different geographical locations or clients. However, there are some exceptions. In certain projects, clients may be using proprietary technology tools and applications. It then becomes critical for the project team to understand the use of such tools and become proficient in using them.

Training in proprietary tools is usually provided by clients and is part of the project cost. Depending on the complexity of the proprietary tools and applications and the extent to which the project team is familiar with their use, the nature and extent of training provision will vary. The second variation in training, albeit of a limited nature, occurs for a small group - the solutioning teams and business analysts who require domain and country-specific knowledge for framing effective solutions.

Project changes are requested in the duration of a project's life cycle. Most requests have a minimal impact on the volume and diversity of training, as there is already a dedicated pool of resources for a technology competency. The scenario changes significantly if a project team working on "packaged applications" such as SAP, Siebel, or PeopleSoft receives requests from the client to upgrade to a new version of the packaged application or product. This project enhancement involves huge upskilling for the team. It entails significant retraining and familiarisation with the new version of the applications' key functionalities, thus affecting the volume and diversity of training.

\section{Strategic HRM Approach, Skills Level, and Workforce Composition}

As noted earlier, externalisation of training and an increasing reliance on graduates reflects a change from the US parent's people management strategy. Organisation E typifies Boxall's (2003) Type 3 firm - a highly differentiated work organisation with evidence of the use of high-performance work practices. The strength of its QMS specifies recruitment standards - people with fewer skills and experience - and identifies training needs in a narrow range of tasks and technology competencies. The above approach was applied at Organisation E, using competency-based skills development and performance management practices. While the above allows for a systematic and standardised approach to skills development, it is through the development of strong QMS that predictability of its service delivery is ensured and, strict process discipline is infused into its large group of relatively young and inexperienced employees. 


\section{Specific Impact of Factors on Nature and Extent of Training}

The following section provides an analysis of how various factors affect the nature (externalisation, diversity, and formalisation) and extent (volume) of training. Following Miles and Huberman (1994), a conceptually clustered matrix was first developed. The conceptually clustered matrix offers a snapshot of the intensity and direction of the different factors and includes notes from the researcher (see Table 10).

\section{Extent of Training}

Apart for the mandated 2 weeks per person per annum training for all employees, Organisation E, on average, invests in $8-10$ weeks of training for new hires. Training volume is directly affected by high employee turnover and the increasing enterprise size and business complexity.

The temporal dimension of a project's life cycle has a limited impact on training volume, as team members are already familiar with a given technologies. The only exception being when some projects involve use of clients' proprietary tools and applications and packaged software services, or when a client requests to move to a newer version of the product/application. Subsequently the demand for nature and extent of training increases. Training volume is also driven by its quality management frameworks (ISO and CMM) as they mandate minimum days of training for all employees.

\section{Nature of Training}

Most of the training is focused on building global IT competencies and is thus technical and mostly transferable in nature. There is, however, some provision of investment in generic people management and leadership competencies and role-based training. As noted earlier, Organisation $\mathrm{E}$ has a huge reliance on external training providers for delivering training. Training on quality management tools and standards is provided to all employees. The quality management frameworks employed also require formalised approaches for evaluation and delivery of training. Its mandated 2-week training focuses more on ongoing career development needs of its employees. 
Table 10.

\section{Conceptually Clustered Matrix of Factors}

\begin{tabular}{|c|c|c|c|c|}
\hline S.No. & Main factors and attributes & Intensity & Respondents & Researcher's notes \\
\hline 1 & $\begin{array}{l}\text { Workplace change } \\
\text {-structural } \\
\text {-ownership } \\
\text {-technological }\end{array}$ & $\begin{array}{l}\mathrm{H} \\
\mathrm{H} \\
\mathrm{H}\end{array}$ & $\begin{array}{l}1,2,3,4 \\
1,2,3,4 \\
1,2,3\end{array}$ & $\begin{array}{l}\text { New technology, changing ratio of graduates to experienced } \\
\text { employees, and use of a competency-based skills development } \\
\text { impacts on training provision. }\end{array}$ \\
\hline 2 & $\begin{array}{l}\text { Market orientation (M0) } \\
\text {-information sensing } \\
\text {-information dissemination } \\
\text {-organisational response }\end{array}$ & $\begin{array}{l}\text { M-H } \\
\mathrm{H} \\
\mathrm{H}\end{array}$ & $\begin{array}{l}1,2,3 \\
1,2,3 \\
1,2\end{array}$ & $\begin{array}{l}\text { Sensing and solutioning is mostly done by US employees. Solutions } \\
\text { are delivered by Indian project teams. }\end{array}$ \\
\hline 3 & $\begin{array}{l}\text { Learning orientation (L0) } \\
\text {-commitment to learning } \\
\text {-open-mindedness } \\
\text {-shared organisational vision }\end{array}$ & $\begin{array}{l}\mathrm{H} \\
\mathrm{M} \\
\mathrm{H}\end{array}$ & $\begin{array}{l}1,2,3 \\
1,2,3,4 \\
1,2,3,4\end{array}$ & $\begin{array}{l}\text { Evidence of critical thinking is present at solutions stage-but } \\
\text { mostly in the US. Strong training infrastructure. }\end{array}$ \\
\hline 4 & $\begin{array}{l}\text { Quality management systems (QMS) } \\
\text {-Commitment \& sharing information } \\
\text {-continuous improvement } \\
\text {-teamwork }\end{array}$ & $\begin{array}{l}\mathrm{H} \\
\mathrm{H} \\
\mathrm{M}-\mathrm{H}\end{array}$ & $\begin{array}{l}1,2,3 \\
1,3 \\
1,2\end{array}$ & $\begin{array}{l}\text { Strong internal quality standards and processes. All processes } \\
\text { documented and mapped on the CMM framework }\end{array}$ \\
\hline 5 & Employee turnover & $\mathrm{H}$ & $1,2,3$ & Varies with business lines and competency areas. \\
\hline 6 & $\begin{array}{l}\text { Enterprise size, complexity and } \\
\text { ownership }\end{array}$ & $\begin{array}{l}\mathrm{H}, \mathrm{H} \& \\
\mathrm{MNC}\end{array}$ & 1 and 3 & $\begin{array}{l}\text { Extremely large and complex organisation; posting high growth } \\
\text { rates in revenues and employee numbers for the last } 4 \text { years; diverse } \\
\text { portfolio, caters to numerous verticals and domains. }\end{array}$ \\
\hline 7 & Temporal dimension & M-L & $1,2,3,4$ & $\begin{array}{l}\text { Apart from the use of clients' proprietary tools, new projects or } \\
\text { project enhancement have limited impact on training. }\end{array}$ \\
\hline 8 & Geographical dimension & $\mathrm{L}$ & 1 and 2 & Minimal training impact on work from different geographical areas. \\
\hline 9 & $\begin{array}{l}\text { Strategic HRM approach and workforce } \\
\text { composition } \\
\text {-rewards and performance management systems } \\
\text {-career planning } \\
\text {-recruitment skills level \& operating roles; and } \\
\text {-use of non-standard employment }\end{array}$ & $\begin{array}{l}\mathrm{H} \\
\mathrm{H} \\
\mathrm{H} \\
\mathrm{L}\end{array}$ & $\begin{array}{l}1 \text { and } 3 \\
1,2,3 \\
1,2,3 \\
1 \text { and } 3\end{array}$ & $\begin{array}{l}\text { Strong competency-based skills development and performance } \\
\text { management. It also supports career development needs. Increasing } \\
\text { reliance on freshers and its learning and development infrastructure. }\end{array}$ \\
\hline
\end{tabular}

Legend: $H=$ High; $M=$ Medium; $L=$ Low; $N=N i l$

\section{Discussion}

It follows from the above analysis that various factors shape the nature and extent of training provision. Organisation $\mathrm{E}$ has medium to high $\mathrm{MO}$ and LO, and strong QMS. 
LO, MO and QMS work synergistically in framing appropriate operational and training responses. The inability to develop a high LO is partly due to the nature of work that it receives from its parent organisation and partly due to the highly structured work organisation. Highly structured work organisation and business processes are enabled by its strong quality management capabilities. The limited opportunity to challenge project specifications that is affecting the development of its LO. Thus, much of the training and development focuses on incremental and adaptive learning (Baker \& Sinkula, 2002) or single-loop learning (Argyris \& Schon, 1978). Strong MO and QMS are strengthening the development of its client-specific capabilities.

Owing to similarities present in a captive offshore business model, the overall demand for the nature and extent of training should decrease (Bartel \& Lichtenberg, 1987). However, technological changes, expansion in its business lines and IT competencies, changing workforce composition, and high employee turnover continue to have a positive impact on the nature and extent of training provided. In terms of the temporal dimension of projects, training provision is effected with the use of proprietary tools and applications by clients and a move to new versions of products in the packaged applications area.

\section{Conclusion}

With increasing costs in factor markets, Organisation $\mathrm{E}$ has to manage its human resources costs, which form a significant proportion of its service delivery costs. Competition in factor markets intensifies as more global IT majors expand or set up their operations in India, not only to tap into the cost arbitrage, but also to compete in product markets globally (using India as one of their development bases). The case study also points to the inadequacies in the Indian engineering curriculum and the pressure the educational system is under to produce an increasing number of readily employable graduate engineer trainees. 


\title{
CHAPTER 5.6
}

\section{ORGANISATION F}

\begin{abstract}
About Organisation F
Established more than two decades ago, Organisation $\mathrm{F}$ is an extremely large, Indianowned, multi-billion-dollar IT organisation employing about 40,000 staff at the time of this study (2005-06). Organisation F has diverse business lines and is organised around the industries and locations that it services. For example, it provides end-to-end business and technology solutions to the following geographical areas in a range of industry sectors: North America, EMEA (Europe, the Middle East and Africa), and Asia-Pacific. Mainly a third-party service provider, Organisation F offers solutions to numerous industry sectors, such as the automobile industry, aerospace and defence, banking and capital markets, education, financial services, health services, hospitality, insurance, logistics, manufacturing, retail, telecommunications, and utilities.
\end{abstract}

Within the above industries, it provides technology and business solutions in the following areas: consulting, applications services, product engineering, custom software development, application and technology maintenance, re-engineering, testing and validation services, IT infrastructure management, and ITeS/BPO services. These areas are further split into sub-specialisations and vary in their nature and extent according to their industry and geography. For example, in the retail industry, the organisation caters to two market segments: grocery and pharmacy. In these market segments, it offers, for example, logistics, data management, performance management, merchandising, sales, application management services, supply chain services, RFID, and packaged application services. Further, within packaged application services, it offers, for example, human capital management services, which focus on HR consulting, HR technology, and outsourcing solutions.

As part of an embedded case study design strategy, this case study provides insights about Organisation F's two business units-one specialising in product engineering services for the telecommunications sector and the other a software product developed for the banking sector. 


\section{Organisation Structure and Processes}

Organisation F's above-mentioned industries and specialisations are supported by enabling functions such as HR, marketing, training, quality and sales. For each geographical market and industry sector, Organisation F has independent business units (IBUs). A typical IBU is headed by a delivery head and is supported by senior consulting and client engagement managers. The majority of its business is from repeat customers, although, as part of its business strategy, business development teams are continuously focusing on developing new customer accounts in existing verticals (industries) and specialisation areas.

In the last few years, Organisation $\mathrm{F}$ has restructured from a functional organisational structure to an industry or vertical-based structure. Expanding its operations in certain industry sectors allows Organisation $\mathrm{F}$ to develop deeper industry and domain-specific knowledge of those industries' competitive environment. Organisation F is a highly process-oriented firm and work is organised around specific role groupings. Out of a total of about 300 distinct roles within the organisation, the delivery group employs the most people. For example, one role - software engineering - has close to 15,000 employees. Other role groupings are smaller and include the client-facing groups (40-50 roles), consulting, enabling, and solutions groups.

\section{Business Processes, Strategy, and Work Organisation}

Being highly process-focused is critical in a third-party global delivery services model, as it enables higher levels of service predictability and better project management. Organisation $\mathrm{F}$ is one of the early adopters of a range of quality management frameworks employed by the Indian IT industry. In the early 1990s, Organisation F secured the ISO 9000 certification, and later it adopted the Malcolm Baldridge National Quality Award framework for improving its organisational management processes. Subsequently, it secured Carnegie Mellon University's the Software Engineering Institute-Capability Maturity Model (SEI-CMM) and CMMI (Integrated) Level 5 certifications to improve its software development and delivery process capabilities. For improving its crossfunctional process mapping and process capabilities, Organisation F further invested in Six Sigma methodologies. 
Considering the compounded annual growth rates of $30-40 \%$ in revenues and employee numbers, adoption of the above QMS enable predictable software development and effective management of its business processes. From the early 1990s until the present, to support its growth plans and the resultant changes in its skills profile in the IT labour market, Organisation F made sustained investments in the development of a large internal technical training and management development infrastructure.

Owing to the increasing competition for skills in factor markets, Organisation F annually analyses and forecasts the skill sets it needs. High rate of skills obsolescence sets and the rising costs of skilled labour have necessitated its sustained investment in its training infrastructure. Some senior managers view that because it is not a zero sum game in terms of the market potential of its services and owing to its current long-term contracts with existing customers, Organisation F is committed to developing a strategy for managing rising labour costs, upskilling employees in new technology areas, and supporting employees' career aspirations and retention. Its business strategy focuses more on the "make" versus "buy" HR decision. A senior learning and development manager explained:

[The] business model is saying how do you leverage? Because, the cost structure ... at the lower level you have to manage the cost structure... So, for instance, the competing company might have people with 5 years experience doing a particular job ...here, we may be [having] somebody with 2 years experience. So, it's the business model which says that leverage the resources as well as you can and provide the necessary support so the driver is the business model.... The strategy really is managing the cost structure, but in order to make it work you have to invest. And so investment is also good for us and there is fast growth for people. So, people appreciate that kind of responsibility, that I can get to [a role] in 5 years; or, if I can get to [a role] in 2 years and with necessary support, then people are happy.

The development of a strong training infrastructure enabled Organisation F to recruit an increasing number of engineering graduates from campus and to train them in a range of technology competencies. It is worth adding that most new engineering graduates have no work experience; most enrol for a four-year engineering degree soon after finishing 14 years of compulsory schooling. The focus of learning at Organisation F is not just on building internal technology-training capabilities, but also on containing the employee costs associated with hiring experienced employees from a tight labour market to undertake entry level software development roles. 


\section{Nature and Extent of Training}

The nature and extent of training provision have become complex since Organisation F's organic growth and its move to a global business delivery model. Organisation F's training can be broadly grouped into four areas: technical, behavioural, process (quality) and domain training. Technical training constitutes the largest volume of training. Organisation F provides three and a half months, or 15 weeks, of technical training to its engineering graduates. Based on its human resource planning forecasts, it trains new hires in a range of technology areas. Most (85-90\%) of the training provided is technical in nature and delivered using its internal training infrastructure. Behavioural training (10$15 \%)$ is mostly sourced internally.

A Bachelor in Engineering completing its 15-week technical training has been evaluated by independent consultants from the US, to be equivalent to a Bachelor of Science (Computer Science) degree from a university in the USA. In addition to the above technical training, Organisation $F$ offers a different stream of training in enterprise solutions, based on the Enterprise University model in the US, which focuses specifically on packaged applications such as SAP, PeopleSoft, and Oracle.

Owing to its large and diversified portfolio, Organisation $\mathrm{F}$ is able to derive economies of scale and scope from its training infrastructure. Its high business growth rate is associated with corresponding growth in employee numbers, which consequently drives its training volume. For example, its current employee base of 40,000 and an annual growth rate of $40 \%$ translate into the recruitment of approximately 16,000 new employees each year. The average employee attrition rate of $9-10 \%$ adds another 4,000 to the above figure. Of the 16,000 employees to be recruited, about half are recruited from engineering colleges and the remaining from the external labour market (people with 0-2 years of work experience). Thus, at the time of this study, the organisation employed between $60-70 \%$ of its workforce with work experience of less than 4 years. Organisation $F$ has forged strong partnerships with numerous engineering colleges (from where it recruits most of its graduates) for their curriculum and faculty development. Some optional programmes have been developed to further the employability of students enrolled at these colleges. The above approach also reduces the training time and costs for those students who successfully complete these optional programmes and Organisation F's selection tests. 


\begin{tabular}{|lllll|}
\hline $\begin{array}{l}\text { New hire } \\
\text { training }\end{array}$ & $\begin{array}{l}\text { Project } \\
\text { \& process } \\
\text { training }\end{array}$ & $\begin{array}{l}\text { Role-based } \\
\text { training }\end{array}$ & $\begin{array}{l}\text { Management } \\
\text { \& leadership } \\
\text { development }\end{array}$ & $\begin{array}{l}\text { Domain } \\
\text { and client- } \\
\text { specific } \\
\text { training }\end{array}$ \\
\hline 15 weeks & 1-4 weeks & $\begin{array}{l}\text { Role-dependent } \\
\text { (ongoing) }\end{array}$ & Ongoing & $\begin{array}{l}\text { Project \& } \\
\text { domain-dependent }\end{array}$ \\
\hline 2005-2006: key focus areas & & & & \\
\hline $\begin{array}{l}\text { New hire } \\
\text { training }\end{array}$ & $\begin{array}{l}\text { Process } \\
\text { training }\end{array}$ & $\begin{array}{c}\text { Role-based skills } \\
\text { \& knowledge }\end{array}$ & $\begin{array}{l}\text { Leadership \& } \\
\text { certification } \\
\text { programme }\end{array}$ & $\begin{array}{l}\text { Domain } \\
\text { expertise } \\
\text { client tools \& } \\
\text { applications }\end{array}$ \\
\hline
\end{tabular}

Figure 20. Temporal dimension of training: Changing emphases.

Figure 20 is an attempt to capture Organisation F's complex and diverse nature of training. Following entry-level training, employees undergo project-specific training. As employees are assigned specific roles in the organisation, they are provided with some role-based training, which focuses on the key role descriptors and the competencies associated with it. For hiring employees with experience (commonly referred to as laterals), the organisation provides orientation training, process training, and role-based training. Although the majority of its training needs are sourced internally, there are a few areas in behavioural and technical training where Organisation F relies on the expertise of specialist training providers, such as McKinsey Consulting, and senior faculty from premier engineering institutes. Organisation's clients also provide training in their proprietary tools and applications.

In the last two years, Organisation $F$ has moved away from the paternalistic approach of taking complete responsibility for employees' career development and growth to one which involves a merit-based "certification" programme for assessing employee knowledge and skills. Under the new approach, employees have to develop and be certified in role-based competencies. The certification system has internal subject-matter experts who carry out role-based competency assessments of people seeking to grow in various roles. This approach encourages employee upskilling and competency development, and assessment focuses on transfer of learning. Certification is carried out at three levels, for example, 100, 200 and 300, where 300 is a mastery level in a competency area. This approach also creates a sense of responsibility and accountability 
for personal development amongst employees; for example, to grow into a project management role, employees must develop, among other competencies, project cost estimation skills and project management competence to a requisite level.

Organisation F's process (quality) training focuses on four main areas: understanding quality management processes and how they are intertwined at various stages of the software development lifecycle (SLDC); review and evaluation checkpoints at various stages of the SLDC; creating multiple artefacts for capturing and configuring various quality processes at different stages of the SLDC; and creating an understanding of its quality management processes and tools, and the underlying philosophy.

Domain training focuses on understanding the clients' business problems and offering technology or business reengineering solutions. Domain training involves an understanding of industry-specific and client-specific business problems in unique geographical contexts. Client-facing and domain competency groups are provided with detailed training on industry sectors and how they change. Position papers are developed for various verticals, and points of view about different technology solutions are shared with those employee groups who are responsible for developing business and technology solutions. Domain training also focuses on developing consulting skills, client relationship management, account leadership and management, and contract negotiations. Organisation $F$ relies on specialist training consulting firms such as McKinsey Incorporated.

As the case focuses on training in two units, namely, in a product (banking software product) and project environment (product engineering projects in the telecom sector), the nature and extent of training within these groups is also explained as follows. In a product environment, in addition to the 15 -week entry-level training, further training is offered for understanding product functionalities and familiarising with key terminologies. The team that supports the development and maintenance of this product needs to be conversant with its programming codes and the customer risks associated with changing the product functionalities. Few product team members undergo training in the banking and financial domains.

In the network engineering project, the training focuses on understanding networking products and networking technologies, and having domain knowledge of the 
telecommunications sector. In addition to the entry-level 15-week training and a further 6-week project-specific training, client-funded training is provided to understand clients' proprietary tools and applications. The differences between a product and project environment are summarised by a product manager as follows:

\begin{abstract}
What happens in a services [project] environment is, at the outset of the project itself, you know what you need. So, from Day 1 ... we ask people to go for a week or 2 weeks training ... and ask them to come back and we also go back to them when we have to fix something, ... what is there ... best way that is possible ... using the best practice guidelines which are available in that particular language.... In a product side of things, we don't really have any such window of opportunity. For example, because ... this is what we really need-a product—but the finer details are at a very in-depth level. Because ...we have so many functionalities available ...so all five guys have to learn about it from the scratch. And it's not one line of code; we have 10,000 lines of programming code, like that ... so, you have to dig around and look for it and create it.... So, once you do two or three times, then only you will know where things are fitting.
\end{abstract}

\title{
Analysis of Factors Influencing Provision of Training
}

\section{Workplace Change}

Organisation $\mathrm{F}$ has witnessed four workplace changes. First, its structural change from a functional to an industry- and geography-based structure has resulted in capability development in the industry sectors and domains where it provides services. Growth by industry sector has also resulted in expansion to, for example, the ITeS/BPO and business consulting IBUs.

The second change is Organisation F's move to a role-based organisation. Consequently, a mandated set of competencies have been developed for each role. Third, its global delivery business model, which has now become mainstream, has resulted in intense competition in both factor and product markets. As result, large organisations such as Organisation F have to focus on developing a sustained supply of skilled human resources and putting in place HR strategies to retain them. Finally, its recent move to certify skills and competencies has increased the formalisation of training, evaluation, and contributed to high volume of training associated with role-based competencies to meet the career aspirations of employees. 


\section{Quality Management Systems (QMS)}

Organisation F's high level of quality management capabilities is evident through its investment in ISO, CMMI Level 5, Malcolm Baldridge accreditations, and Six Sigma methodologies. The above frameworks focus on providing defect-free and predictable software services at competitive costs and building process maturity. These frameworks also strengthen its role-based competency mapping and process definitions for each role. The organisation has established a set of best practices for software development at various stages of the software development lifecycle. Its QMS processes are applied during contract negotiations and business requirement specifications stage to incorporate clients' unique needs. The differences between Organisation F's QMS and other organisational capabilities and its clients' capabilities offers Organisation F an opportunity to influence its clients' strategic milieu. Consequently, it helps in securing more business or better service delivery rates (Banerjee, 2004). Organisation F's senior quality manager noted:

See, Organisation F is a very high maturity organisation, and most of our clients are not reaching that level of maturity in terms of processes. Their business maturity is definitely there, but [they] are weak in their software process maturity... Most of the times we are far ahead of the client. So, we try to map. If there is an extra requirement in terms of documentation or reports, then we try to customise it according to the client needs... So, if there is a specific requirement of mapping and training, then this [quality] group will take care.

The cost of implementing quality is very high and impacts service performance and training costs significantly. A senior quality manager stated:

\footnotetext{
The cost of quality in an IT industry is very high and, unbelievably, around $35-40 \%$. The cost typically comprises three aspects: one is the prevention cost, second is the appraisal cost, and third is the premium cost. Prevention cost would typically look at something like training..... Appraisal cost is when we redo; when we detect defects ... reworking happens during the development of the software. Third [premium cost] is after we shipped the software and the customers find the defect or during customer testing.... Those kind of costs are extremely high to absorb, so if you look at the breakup of these ... the majority portion—of [about] 35\% —-would be appraisal.
}

Use of quality management processes and a team-based structure allows for better control of workflow and, enables software development work to progress in a structured and 
predictable manner. QMS focuses on process improvement and productivity gains by sharing established processes as per CMMI Level 5 requirements. These processes infuse process discipline in its young and relatively inexperienced workforce. Process discipline is implemented by allocating quality management resources to service delivery units. These quality resources not only ensure process compliance, but also develop quantitative metrics at various stages of the project. A quality manager clarified:

We are involved at the proposal stage, to tell of quality and look at specific quality requirements in a project, and how they are going to handle quality requirements. We are involved in developing metrics for various levels of performance of the project.

The above views point towards the linkages between a firm's QMS and MO. In both product and project environments, there was evidence of continuous improvement efforts. The nature and extent of such improvements varied and were influenced partly by the risks associated with such improvements and the degree of clients' willingness to accept the change. QMS enable a structured way of sharing metrics and implementing continuous improvement, and act as a catalyst for developing a firm's LO ability, especially its ability to challenge its current theory-in-use (Argyris \& Schon, 1978). The following section explains its MO and LO capabilities.

\section{Market Orientation}

Overall, Organisation F has high levels of MO. Organisation F's sales and client engagement groups are responsible for developing new client accounts through its market intelligence reports and domain expertise. When a new client account is added to its portfolio, the sales team hands over the account to the client engagement team for full account leadership and relationship management (after about 12-18 months of managing the account). The focus shifts from sales to holistic client problem solving. Thus, the organisation's client-facing employees are provided with specialist sales and domainspecific training from external training consultants. The client-facing groups also receive informal coaching and mentoring to develop their business consulting and solutioning skills.

Organisation F's sales team senses client information through RFI and RFP (requests for information and proposals) from new and existing clients, market intelligence reports, and general business and technology trends. Information dissemination occurs at various 
levels. First, the business and technology solutions teams and client-facing groups disseminate information to the delivery teams, wherein all specifications related to the project are shared with respective delivery groups. Second, at a domain level, delivery groups disseminate knowledge about client experiences, testimonials and common problems when dealing with specific business and technology requirements.

This information exchange is formal and documented as best practices, but there are other informal forums for sharing information. At a client account level, the team shares common issues and develops a better understanding of the clients' business model to propose specific responses and solutions using established metrics and QMS processes. The extent to which such information is sensed and disseminated from clients has an impact on the nature and extent of training, more so in the services environment and, to a lesser extent in product environment. One project manager observed:

\footnotetext{
There are client-specific processes ... we have that and, typically, that is done by people in the client group itself. You take your client like ... Bank of America or whatever, so we have quite a few people who are already working with those clients. So, in those cases they would train.... Even ... coding standards, for instance, the way you code for one client, they would have their own internal standards and that has to be understood.... Most of the time, the client would do the first round of training for these people. There is a knowledge transfer if you are working with a client. Then, whatever practices they have, they train you on that. There is a boot camp and they train you on that... That's done as a routine thing. It is quite standard.
}

On the contrary, in a product environment, the training is for the client where the application is to be installed and or supported. The development and maintenance teams are highly skilled and experienced through continuously working on a single product. The nature of knowledge about the product is tacit and internalised. It cannot be formally disseminated to the team members. Although there is initial formal training and structured directories and programme codes available for team members, nevertheless, there is a strong focus on informal learning, especially by "learning by doing" and "finding it out for yourself".

\section{Learning Orientation}

Overall, Organisation F has medium to high levels of LO. There is no doubt a strong commitment to learning is evident through its elaborate learning and development infrastructure and programmes offered. Given its industry and geography-based structure, 
information sharing is strong in the project environment, where the domain- and technology-specific forums are used for sharing best practices. However, in a product environment, there are no formal mechanisms for sharing learning. Learning is mostly informal and incidental in nature (Watkins \& Marsick, 1989), and employees learn in their own time through product dictionaries, indexed materials, and team members.

Different approaches are evident in product and project environments with respect to challenging clients' assumptions and critical thinking. In a project environment, teams have to work to pre-determined solutions. Open-mindedness is possible at the early stages of business requirements specification and negotiations. Later, there is limited latitude in terms of what is to be developed and how. Software development at Organisation F follows clearly defined and mapped processes and uses its QMS templates of best practices for implementing process improvements. Its quality management systems enable the implementation of such structured and process-focused software development.

In a product environment, although there are well-documented processes for writing codes and documenting them, employees are encouraged to consider continuous improvement and reduce product complexity. Product innovation is evident, albeit to a limited extent. A product manager noted:

\footnotetext{
See, innovation needs chaos; that is not acceptable! What we say is that innovation needs planned chaos. Planned increments or planned steps.... Innovation has to happen and innovation has to be thought through. See, we are dealing with real clients for whom we have to supply real products and services. By saying that I am innovative and then there is a huge risk of impacting a particular client, I don't think that any company would support that.... If I am saying that I am going to develop a tool which is going to improve something or reduce the process time or risk or increase the productivity, then consider that. But innovation does not mean that we will do whatever we want.... It's about how doing this will make it better. It can be radical, but that is fine as long as you understand the risks involved.
}

\section{Employee Turnover}

Organisation $\mathrm{F}$ has lower than average industry employee attrition rates, ranging between 9 and $11 \%$ per annum. The bulk of its employee resignations are by employees with 4-6 years of experience, as there is a strong demand for experienced IT professionals, especially those who have worked at Organisation F. "Organisation F brand" commands a 
premium in the external labour market. There is also some turnover at entry level, resulting either from a mismatch between expectations and compensation offered or the intention to pursue higher education. Owing to its large employee base, even $9 \%$ annual employee attrition rate translates into a higher volume of training provision.

\section{Enterprise Size, Complexity, and Ownership}

Organisation F's organic growth rate in employee numbers and business domains, and increasing service complexity have had a positive impact on the nature and extent of training provision. One HR manager confirmed: "The business model has become more complex. So what's it means is that you have to keep providing the training." Indian ownership per se does not have any significant impact on the provision of training; instead, it is the business model of managing costs and its increasing business complexity, which has had a profound impact on investment in training.

\section{Temporal Dimension of Products and Projects}

Further analysis suggests that at different times in a project or product's life cycle, the nature and extent of training required changes. In a project, the training volume and diversity is high at the start. Project-specific training is provided to all new employees, especially when the project is in a new domain or from a new client. A project manager (Network Engineering_-Telecom) commented:

\footnotetext{
Say one domain to another.... OK, I mean customer-specific training is also required ... if I go from Nortel to Lucent the domain was same-it was telecom domain. But, the architecture of that software and the hardware architecture ... the capacity, how you quantify, how you test those systems - test tools which are used to test the systems - those things are more or less different. ... They [clients] are the guys who know. They have the expertise in the area and provide us training.... It is not where we would say [we] have a standard Organisation F infrastructure.... I mean, we didn't per se pursue domain training ... technology training or the platform training because those are some things which we know that it will take us a long time [to] get through. We need to have customer equipments here.
}

On the contrary, in a product development environment, training needs are low in the initial stages of product development as the core development team is coding and developing the product functionality. The development team follows an informal and 
iterative cycle of developing the product. Initially, there is no formal training necessary, except maybe to familiarise a new employee with the technology used by the application. The need for training increases subsequently, when the product is released. Product version change, whether minor or major, results in additional training volume. Training is needed for the remaining product team members and the clients using the application. Clients' customisation requests do not have any impact on training needs because the product development team is conversant with the code.

Ongoing role-based career development demands investment in training for supporting the development of role-specific competencies. For employees to advance to different roles, they need different sets of training-from technical to business and behavioural skills development. For example, senior client-facing groups undergo customer relationship management and diplomacy skills training.

\section{Strategic HRM Approach, Skills Level, and Workforce Composition}

Organisation $\mathrm{F}$ shows strong evidence of a strategic approach to managing its human resources and skills development. While most (80-85\%) of its workforce skills requirements can be forecasted owing to its long-term contracts with repeat customers and its understanding of where future business growth will come from, some (15-20\%) just-in-time hiring cannot be ruled out.

Organisation F's learning and development strategy typifies a "make" instead of a "buy" decision. The focus is on managing costs and ensuring a sustained supply of IT professionals. The above approach is reflected in its recruitment and selection strategies (of recent college graduates and people with $0-2$ years of work experience) and the nature of employment (mostly [96\%] full-time permanent, with limited reliance on nonstandard forms of employment). Based on its learning and development facility's capability to train graduates with a certain level of academic achievement and performance on the entry test, it has a fairly low selection ratio. Depending on the institution, the cut-off percentage and test scores are modified, so as to get, as far as possible, a uniform cohort of trainable employees. To support the career development needs of its staff and to retain talent, Organisation $\mathrm{F}$ follows a role-based competency development approach. 


\section{Specific Impact of Factors on Nature and Extent of Training}

The following section provides an analysis of how various factors affect the nature (externalisation, diversity, transferability, and formalisation) and extent (volume) of training. Following Miles and Huberman (1994), a conceptually clustered matrix is developed. The conceptually clustered matrix offers a snapshot of the intensity and direction of the different factors (see Table 11) and includes notes from the researcher.

\section{Extent of Training}

Most training volume is driven by Organisation F's business strategy of managing its cost structure by having a high percentage $(60-70 \%)$ of new hires (people with $0-4$ years of experience) in its workforce. Training volume is also affected by organisational change, employee turnover and the organisation's growth in size and diversification to new business lines. The temporal dimension of a project's life cycle on training volume has a limited impact, provided the technology and domain remains constant. Common exceptions include the use of clients' proprietary tools and applications or, in a product environment, the launch of a new product version. The application of QMS impacts significantly on process training, and the use of quality management tools and best practice guidelines impacts significantly on the volume and diversity of training.

\section{Nature of Training}

Business complexity, service diversity, QMS, and organisation size impact on the diversity of training outcomes. Formalisation and diversity of training are influenced by the use of its quality management tools, organisational change to a role-based competency certification for career development, and implementation of other mandated training requirements under various quality frameworks. Organisation F has limited reliance on external training providers; only specialist training is outsourced. Most training is technical in nature and the need for behavioural training increases for roles with higher responsibility. 
Table 11.

\section{Conceptually Clustered Matrix of Factors}

\begin{tabular}{|c|c|c|c|c|}
\hline S.No. & Main factors and attributes & Intensity & Respondents & Researcher's notes \\
\hline 1 & $\begin{array}{l}\text { Workplace change } \\
\text {-structural } \\
\text {-ownership } \\
\text {-technological }\end{array}$ & $\begin{array}{l}\mathrm{M} \\
\mathrm{H} \\
\mathrm{M}\end{array}$ & $\begin{array}{l}1,3,4,6 \\
1,3,6 \\
1,2,3,4,5,6\end{array}$ & $\begin{array}{l}\text { New technology, ratio of new hires to experienced } \\
\text { employees, and use of role-based competency development. }\end{array}$ \\
\hline 2 & $\begin{array}{l}\text { Market orientation (M0) } \\
\text {-information sensing } \\
\text {-information dissemination } \\
\text {-organisational response }\end{array}$ & $\begin{array}{l}\mathrm{H} \\
\mathrm{H} \\
\mathrm{H}\end{array}$ & $\begin{array}{l}1,3,4,6 \\
1,3,4,6 \\
2,3,4,5,6\end{array}$ & $\begin{array}{l}\text { Sensing and solutioning mostly done by client-facing groups } \\
\text { in different verticals. Solutions are developed and passed on } \\
\text { to delivery teams. }\end{array}$ \\
\hline 3 & $\begin{array}{l}\text { Learning orientation (L0) } \\
\text {-commitment to learning } \\
\text {-open-mindedness } \\
\text {-shared organisational vision }\end{array}$ & $\begin{array}{l}\mathrm{H} \\
\mathrm{M}-\mathrm{H} \\
\mathrm{H}\end{array}$ & $\begin{array}{l}1,3,4,6 \\
1,2,4,5,6 \\
1,2,5,6\end{array}$ & $\begin{array}{l}\text { Evidence of open-mindedness is present but to a limited } \\
\text { extent. Strong focus on solutions delivery and internal } \\
\text { process improvements. }\end{array}$ \\
\hline 4 & $\begin{array}{l}\text { Quality management systems (QMS) } \\
\text {-Commitment \& sharing information } \\
\text {-continuous improvement } \\
\text {-teamwork }\end{array}$ & $\begin{array}{l}\mathrm{H} \\
\mathrm{H} \\
\mathrm{M}-\mathrm{H}\end{array}$ & $\begin{array}{l}1,2,3,4,5,6 \\
1,2,4,5,6 \\
1,2,4,5\end{array}$ & $\begin{array}{l}\text { Well-developed internal quality standards and processes. All } \\
\text { processes documented and mapped according to its quality } \\
\text { management frameworks. }\end{array}$ \\
\hline 5 & Employee turnover & H to M-L & $1,2,3,6$ & Varies with business lines and competency areas. \\
\hline 6 & $\begin{array}{l}\text { Enterprise size, complexity and } \\
\text { ownership }\end{array}$ & $\begin{array}{l}\mathrm{H}, \mathrm{H} \text { and } \\
\text { Indian }\end{array}$ & $1,2,4,5,6$ & $\begin{array}{l}\text { Extremely large and complex organisation; high growth } \\
\text { rates of employee numbers. }\end{array}$ \\
\hline 7 & Temporal dimension & $\mathrm{M}-\mathrm{H}$ & $2,5,6$ & $\begin{array}{l}\text { Apart from the use of clients' proprietary tools, new projects } \\
\text { or project enhancement have a limited impact on training. }\end{array}$ \\
\hline 8 & Geographical dimension & $\mathrm{L}$ & $2,5,6$ & $\begin{array}{l}\text { Minimal training impact as competency development is } \\
\text { mostly offshore and technology cuts across borders. }\end{array}$ \\
\hline 9 & $\begin{array}{l}\text { Strategic HRM approach and workforce } \\
\text { composition } \\
\text {-rewards and performance management systems } \\
\text {-career planning } \\
\text {-recruitment skills level \& operating roles; and } \\
\text {-use of non-standard employment }\end{array}$ & $\begin{array}{l}\mathrm{H} \\
\mathrm{H} \\
\mathrm{H} \\
\mathrm{L}\end{array}$ & $\begin{array}{l}1 \text { and } 3 \\
1,2,3,5,6 \\
1,2,3,5,6 \\
1,2,6\end{array}$ & $\begin{array}{l}\text { Strong role-based competency development to support } \\
\text { career development needs. Strong reliance on new hires and } \\
\text { use of its learning and development infrastructure. }\end{array}$ \\
\hline
\end{tabular}

Legend: $\mathrm{H}=$ =High; $\mathrm{M}=$ Medium; L=Low; $\mathrm{N}=\mathrm{Nil}$

\section{Discussion}

Organisation F's high software development process maturity is due to its strong quality management capabilities and experience on process improvisations. Organisation $\mathrm{F}$ has medium to high LO, which is stronger for its product division (planned innovation) and 
relatively less developed for its project division (predetermined solutions for service delivery). The less developed LO in services does not indicate a lack of ability to challenge clients' assumptions, but a lack of opportunity from clients and the typical nature of work received.

Its medium to high MO is well developed for its project (services) division (owing to tight project management and the strong customer relationship capabilities developed through repeated interactions with existing clients) and is relatively less developed for its product division (owing to a product superiority mindset). Its' MO and LO, together with its strong quality management capabilities, enable systematic responses to project delivery and learning needs.

Thus, the resultant learning from its training and development programmes results more in incremental and adaptive or single-loop learning, and to a limited extent in doubleloop learning (Argyris \& Schon, 1978; Baker \& Sinkula, 2002). Although repeat business enables the development of client-specific capabilities, Organisation F's reorganisation requires development of industry-specific domain expertise, critical for engaging in higher-level learning and service delivery. Its highly differentiated business model, workforce composition, strong learning and development infrastructure promotes a developmental approach to managing its human resources (Ashton \& Sung, 2006; Boxall, 2003).

\section{Conclusion}

This case provides an understanding of how a large ITSS organisation leverages its learning and development and QMS infrastructure to overcome competition in factor markets, maintain its cost advantage, and offer highly differentiated services at the same time. The case also points to the inadequacies in the engineering educational curriculum, and the role QMS play in infusing process discipline into software development and business processes, to enable the implementation of large-scale people management strategies for a relatively young and inexperienced workforce. Finally, the case demonstrates how high process maturity, industry-specific domain knowledge, and technology capabilities can be leveraged to deliver sustained levels of performance and growth. 


\title{
CHAPTER 5.7
}

\section{ORGANISATION G}

\begin{abstract}
About Organisation G
Organisation $\mathrm{G}$ is an offshore development centre (ODC), wholly owned by a large USbased semiconductor organisation manufacturing microprocessor products. Organisation G's US parent set up the Indian ODC in 1999. At the time of the study (January, 2006), it employed 2,700 employees. Organisation G's parent firm has been a market leader in the microprocessor market for nearly four decades and has a strong portfolio of microprocessor and related products. The Indian facility is a research and development $(\mathrm{R} \& \mathrm{D})$ and design centre and is organised around four key technology groups:
\end{abstract}

- mobility solutions

- digital enterprise solutions

- software solutions

- internal solutions technology.

The number in each of the above groups varies according to the yearly work plans rolled out from the US, the ODC's competencies, and the nature of R\&D and design work to be undertaken. The above technical groups comprise about $85 \%$ of its workforce. Sales and marketing constitute another $10 \%$ of its workforce. They are tasked with selling Organisation $G$ brand products to a range of industry sectors in India, such as telecom, computer hardware, and computer software development. The remaining $5 \%$ of the workforce is in enabling and support functions, such as HR, legal, finance, and corporate services. This case study focuses on mobility and software solutions groups.

\section{Organisation Structure and Processes}

Organisation $\mathrm{G}$ follows its global work structure, whereby each business unit has two business heads for ensuring business continuity planning. Each group head reports to the country head, and the group's regional head. For example, Organisation G's HR head reports to India's country manager and the Asia-Pacific head of HR. 
To support the above groups and implement its global work culture, Organisation G closely follows its global set of values and behaviours. Its global values (customer orientation, discipline, quality, risk taking, great place to work, and results orientation) and work behaviours (assumed responsibility, achievement awards, constructive confrontation, community responsibility, continuous improvement, copy exactly, disagree and commit, measurement focus, meritocracy, and effective meetings) help to reinforce the parent organisation's work culture at Organisation G. Some of these values and behaviours were observable during the field visit.

\section{Business Processes, Strategy, and Work Organisation}

Workflow is determined by the long-range strategic planning cycle in the US. The product development cycle follows on from the planning cycle, and annual and quarterly targets are determined for Organisation $\mathrm{G}$ to deliver. New product development normally takes between 12 and 18 months. Thus, quarterly targets are set for each stage of product codevelopment. In some cases, the entire product is developed by the Indian ODC. In most other cases, there is collaborative codevelopment between the ODC and one or more global sites. Product development is managed by what Organisation G calls an “embo" approach (MBO-management by objectives), wherein development targets and metrics are captured and reported at the end of each quarter.

Detailed level of planning and scheduling ensures predictability and tight monitoring of targets for each quarter. Keeping in mind the core competencies of Organisation G's work site, codevelopment plans are developed between various sites and Organisation G. One of the core competencies identified for the Indian ODC is validation services.

Being a market leader for nearly four decades, the US parent organisation has welldeveloped channels for sensing information through its market intelligence reports and customer feedback. The US parent organisation has for a long time followed a timepacing strategy of product development. Such a strategy involves developing highly granulised performance metrics based on time and synchronising the launch of new products in a manner that enables smooth transition from one product to another. Owing to the market leadership that it enjoys, Organisation $G$ has been able to determine and define its competitive landscape by a phased introduction of higher end versions of 
microprocessors and other products. However, its technology leadership is now being questioned by its customers' changing needs. One training manager noted:

\begin{abstract}
We have to be more customer-focused. Earlier we didn't want what they [customers] wanted. Earlier, you wanted 500 megahertz; we gave you 600 megahertz in 18 months. I think people who need to buy ... now clock speed is irrelevant... Certainly, customers do not find sufficient usage from the product, so now people are looking for applications where it can be used. We need to define a platform for it; this is what Organisation G will do in future.... We are trying to work on new architectures and platforms relevant to the market, which means, up till now, we were focusing on clock speed and ... Moore's law prevailed. But now Moore's law is not important, microprocessor speed is not important, but what customers need is. So, the technology-excellence focus is being shifted to customer requirement focus, which is a paradigm shift.
\end{abstract}

In the US, as part of its people management strategy, a significant proportion (about $70 \%$ ) of its workforce comprises recent college graduates (RCGs), which allows it to manage its labour costs. One HR manager noted:

\footnotetext{
There is a certain amount of slack built into the resourcing model to allow for the training and downtime. The productivity of Organisation G [parent] is relatively less than XXX [its nearest competitor]. For example, they [the competitor] will have about 300 people working on a processor, Organisation $\mathrm{G}$ will have about 150, and their average years of experience will be 6-7 as compared to Organisation G's 2-2.5 (years). This slack is built into the costing. It's a leader and follower strategy.... It's a very carefully thought-out strategy. If you are leading the technology, then you are not competing against somebody; you are not really in a rush to complete a project. You have an immediate head start over your nearest competitor. This strategy has worked really well for Organisation G globally.
}

The Indian ODC, on the contrary, employs only $20-25 \%$ RCGs in its workforce. Being a relatively new ODC and having an evolving learning and development infrastructure, Organisation $G$ had to initially rely more on experienced workers than on RCGs. However, the mandate now is to increase its percentage of RCGs to the total workforce.

\title{
Nature and Extent of Training
}

Organisation G's training infrastructure is split into two main groups: training needs analysis and logistics. While the former focuses on developing training to meet its business group needs, the latter focuses on logistical arrangements, such as room and 
trainee bookings, promotional materials, and evaluation. Most training is technical in nature and can be divided into four key areas: integration (induction and orientation), post-integration, management and leadership development, and organisation development interventions.

Integration training focuses on understanding Organisation G's (parent's) strong work culture and the processes embedded in its work routines, developed over the last four decades. Additionally, Organisation G's site-specific training programmes offer to accommodate the Indian cultural environment and the specific nature of work the organisation receives. All RCGs are first assigned buddies to learn Organisation G culture. Trainees need to familiarise themselves with the use of a highly acronym-centric language (which was observable during the visit). Subsequently, they undergo business technical training, which is group-specific.

Post-integration training focuses on project-specific training in each business group. Additionally, it focuses on developing project management and semi-consulting skills, developing a global mindset, and developing volunteers to become instructors. Organisation G has a strong global Voluntary Instructor Programme (VIP), which focuses on developing internal resources for delivering Organisation G University courses.

Organisation G University has about 7000 courses available online to all global sites. Most of the programmes are delivered by local instructors (internal and external) and with help from US-based subject matter experts (SMEs). There is a strong preference in the Indian cultural setting to learn in a classroom environment. Consequently, Organisation $G$ uses mainly external trainers, US SMEs, and course content from Organisation G University for different types of training. SMEs in specialist areas update Organisation G University's content based on their project experience and feedback from market intelligence reports. Organisation G University is similar to Taylor and Patton's (2002) classification of a "polymorphous" university, and the above approach to leveraging internal learning and development capabilities is consistent with a resourcebased view of the firm (Barney, 1991).

Organisation G's management and leadership development focuses on developing project management skills, increasing people management capabilities, stakeholder management, 
and communicating Organisation G values and behaviours. As the Indian ODC is relatively new, various groups are continuously reorganising themselves to develop a site-specific (ODC) hierarchy and reflect the global changes in its business groups. Such reorganisation and contextualisation of key business processes is enabled by the fourth aspect of training - its organisational development (OD) interventions. While the focus of OD interventions is mostly on problem solving and improvement of business processes, some OD interventions do result in training initiatives.

The bulk of the training $(80 \%)$ provided is technical and utilises internal resources (Organisation G University course content and subject matter experts from various sites) and some external trainers. The remaining $20 \%$ of training focuses on developing behavioural, soft skills and management and leadership development, using mostly internal content and resources. Indian cultural conditions and the ODC's unique hierarchy requires contextualisation of some Organisation $G$ University content, utilising a combination of structured and unstructured learning strategies for delivery.

\begin{tabular}{|lllll|}
\hline $\begin{array}{l}\text { Integration } \\
\text { training }\end{array}$ & $\begin{array}{l}\text { Post-integration } \\
\text { training }\end{array}$ & $\begin{array}{l}\text { Project } \\
\text { \& group } \\
\text { training }\end{array}$ & $\begin{array}{l}\text { Management } \\
\text { \& leadership } \\
\text { training }\end{array}$ & $\begin{array}{l}\text { OD } \\
\text { interventions }\end{array}$ \\
\hline 2-4 weeks & 4-6 weeks & $\begin{array}{c}\text { Project/product } \\
\text { dependent }\end{array}$ & $\begin{array}{l}\text { Role-dependent } \\
\text { \& ongoing }\end{array}$ & $\begin{array}{l}\text { Problem } \\
\text { dependent }\end{array}$ \\
\hline (TIMELINE DEPENDENT ON THE NATURE OF PROJECT AND BUSINESS \\
2006: key focus areas & GROUP NEEDS) & \\
\hline $\begin{array}{l}\text { New hire } \\
\text { integration }\end{array}$ & $\begin{array}{l}\text { Certification, } \\
\text { project } \\
\text { management }\end{array}$ & $\begin{array}{l}\text { Project \&product } \\
\text { knowledge }\end{array}$ & $\begin{array}{l}\text { Mandated \& } \\
\text { role-specific } \\
\text { development }\end{array}$ & $\begin{array}{l}\text { Problem-based } \\
\text { structural \& } \\
\text { process } \\
\text { interventions }\end{array}$ \\
& & & & \\
\hline
\end{tabular}

Figure 21. Temporal dimension of training: Changing emphases.

Owing to high project and business group specificity, significant training occurs on the job and has a strong "learning by doing" component. Figure 21 offers a brief overview of the temporal dimension of training. 


\section{Analysis of Factors Influencing Provision of Training}

\section{Workplace Change}

Since its inception in 1999, Organisation $G$ has undergone numerous structural and business group reorganisations to support its global reorganisation plans and to develop a site-specific hierarchy, which is based on the ODC's core competencies. There are two key transitions taking place within Organisation G. First, there has been a shift in the centre of activity from the US to regional sites. Earlier, $80 \%$ of employees were based in the US; now this figure has reduced to $50 \%$, which has had an impact on integration training and has led to the emergence of site-specific programmes.

The second change is a move from its reliance on Microsoft applications and a personal computer market to opportunities in consumer durable and mobility goods, thus requiring the development of a new set of technical competencies. Additionally, and more significantly, the organisation is trying to reinvent its structures and processes to move from a strong product mindset to platformisation. A training and development manager noted that such a change will have various effects.

In fact, I just came from a meeting a few months ago where we had given 70 million dollars flat and asked what is it that Organisation G needs to do differently. We are still trying to work that out. We will have to see [what] competencies that we had earlier that are not adequate now. We need something more now.

The above move will require a change from a strong product mindset (resulting from its product superiority and market leadership) to meeting customers' explicit and latent needs. Training will have a major role to play in developing strong customer orientation and aligning its business and technology processes to the above change.

\section{Quality Management Systems (QMS)}

Organisation $\mathrm{G}$ does not have any formal quality management accreditation, but over the last four decades has developed its own internal quality standards. Organisation G's values (quality and results orientation) and behaviours (copy exactly, continuous improvement, and measurement) typify TQM content and promote the development of its unique quality management metrics, standards, and culture. The role of Organisation G's 
QMS is to understand, document, define, measure, improve, and assess various processes. Organisation $\mathrm{G}$ has highly detailed and defined process metrics and standards for both mobility and software business groups. High levels of customisation and firm specificity in its processes can be viewed as a source of its competitive advantage (Barney, 1991). One HR manager commented:

The tools and standards are so customised to Organisation $G$ that they may not be usable for other companies in microprocessor technologies. [The] level of customisation is exceptionally high. For example, Organisation G was using PeopleSoft's Version 7. When they wanted to move to the next version, Organisation $G$ asked for close to 15,000 customisation requests [so] that it became unviable for PeopleSoft to deliver the customisations to Organisation G. You can imagine what happens in the product development space. That is how the hiring strategy works-[the] right qualification, aptitude and skills, and then they mould them into Organisation G-specific requirements.

Organisation G's QMS focus on standardisation and improving productivity. Its quality manager observed:

\footnotetext{
Basically we have to improve the bottom line, like focusing on cutting costs! Now, the improvement in the cost is the basic requirement. Whatever you do, if the cost does not reduce it doesn't make sense.... And to do that, we come from a process angle. Establishing the process, check how many defects are coming, improve the process ... so that the defects get reduced. Once the defects get reduced, cost is reduced.
}

The above points illustrate that QMS enable the implementation of differentiation and cost leadership strategies.

\section{Market Orientation}

Organisation G's business development and customer information sensing systems are based in the US. End-user interaction is at a high level, and even the architecture and R\&D people in the US do not have direct customer interaction. However, owing to its strong process orientation and established internal performance standards, its information sensing and dissemination ability is strong. Its strong product leadership mindset prevents it from developing a high level of MO. This is gradually changing, but it is still to be seen how it frames its technological and business process responses to meet customers' needs. 
Its response abilities at a site level are largely dependent on strategic planning in the US. In a codevelopment environment, based on information sensed and disseminated and resource mapping, skills development budgets for various projects are determined collaboratively and responses are framed. Despite the strong top-down planning culture, the Indian site has influence on what can be delivered, as there is limited bandwidth of microprocessor-specific skill sets in the Indian labour market.

\section{Learning Orientation}

Organisation $\mathrm{G}$ has very strong LO. Its strong LO levels are evident in its extensive commitment to learning and development infrastructure, a strong culture of supporting learning. Organisation $\mathrm{G}$ also has numerous sites, business groups, and organisational forums for information sharing and developing a shared understanding of key performance metrics for each business group. Additionally, its business values (risk taking and quality) and behaviours (disagree and commit and constructive confrontation) create an environment conducive to product innovation and open-mindedness. At the time of the visit, employee innovations were observable, as they were well displayed through dashboards and online communication sites. Organisation G's quarterly planning cycles, matrix structure, and regional and site reporting relationships ensure constant review and information sharing within and across the organisation.

The extent to which Organisation G can challenge clients' assumptions is dependent on the nature of the projects it receives and the extent to which collaborative codevelopment plans are agreed. If the Indian ODC is leading the project, there is significant scope for critical assessment at the early stages of the project. However, in codevelopment, where other sites take a lead, Organisation $G$ receives pre-determined work plans with tight time schedules, work specifications, and delivery metrics based on long-established quality process standards and benchmarks. Such projects do not offer enough opportunities for engaging in higher level learning, except in improving the internal productivities.

\section{Employee Turnover}

Organisation $\mathrm{G}$ has a lower than average annual employee turnover rate, at $15 \%$. In the mobility group it is as low as $8 \%$, partly owing to a relatively small external market for such specialist skill sets. The employee turnover is less in the $0-2$ years work experience group, higher in 2.5-3 years experience group and highest in the 5-6 years work 
experience group. The above attrition rate is a reflection of the premium the external labour market offers to a specific set of skills and work experience. The above attrition rates do not significantly affect the volume and diversity of training.

\section{Enterprise Size, Complexity, and Ownership}

High-end computing technologies, the associated high-end nature of skills needed for the job, and inadequacies in the Indian educational curriculum for the semiconductor industry necessitate highly focused investment in technical training. Organisation G's ethnocentric approach to managing its culture and recruitment strategy impacts variously on the nature and extent of training. The strong organisational culture of Organisation G's parent requires higher investment in training in the integration programme for the new hires. The focused recruitment strategy (of recruiting more IT professionals with experience) reduces, to some extent, the diversity and volume of training. However, with its recent mandate to gradually increase its current workforce composition of recent college graduates (to levels comparable with those in the US), the organisation is likely to invest more in the nature and extent of training through its employee integration and postintegration programmes.

\section{Temporal Dimension of Projects}

In a product codevelopment environment, the volume and diversity of training are

affected by the nature of the codevelopment work received. In most cases, the volume of training is high when the project starts, but then it tapers off. A training manager noted:

But there is a lot of training whenever there is a new project that's been given here. The person who will be there [from the US] will come down here [to India] and offshoring the project will require that additional bit of training.

However, when the Indian ODC takes a lead in product development, the demand for training is low because the core product development team learns through the project experience of key personnel from the US, and during product development itself a lot of learning-by-doing happens. Subsequently, the training needs for the rest of the development team increase. The training volume and diversity for the development team also increase if a major product enhancement is released. 


\section{Strategic HRM Approach, Skills Level, and Workforce Composition}

High-level people management plans cascade down from centralised annual strategic planning in the US. Organisation G's performance management systems follow an MBO approach, with well-established metrics for measuring performance. Because the Indian ODC's learning and development infrastructure is still evolving and to ramp up its R\&D and design centre operations, Organisation $G$ decided to initially recruit experienced employees from the external labour market. However, the US now has the expectation that at any given time RCGs should comprise at least $30 \%$ of the workforce in India, to help manage employee costs and reduce employee turnover rates.

The above change requires constant improvisation and development of the integration and post-integration programmes. However, the expectation of increasing RCGs is problematic for the Indian ODC for two reasons. First, it does not have a highly experienced resource pool to train for a 6-month integration programme. Second, there is a small percentage of engineering graduates who are suitable for working in semiconductor industry. One recruitment manager commented:

\footnotetext{
No, we haven't seen value lately and, like I said, we don't have [an] integrated course for them to come here and do a programme. We don't have a long-term integration programme, so we can't afford to take people with backgrounds that don't really match. Because we don't have the bandwidth to invest in getting them to learn the basics of the role, we expect them to come with some basics. .... If they don't have the basics, if they are coming from a B-Tech in textiles from IIT [Indian Institute of Technology], then they are not relevant to us. We'd rather go to a local college and hire a computer science person ...or Electrical, Electronics and Telecom, who has the basic grounding.
}

\section{Specific Impact of Factors on Nature and Extent of Training}

The following section provides an analysis of how various factors affect the nature (externalisation, diversity, transferability and formalisation) and extent (volume) of training. Following Miles and Huberman (1994), a conceptually clustered matrix was first developed. The conceptually clustered matrix offers a snapshot of the intensity and direction of different factors with notes from the researcher (see Table 12). Subsequently, explanatory maps were developed to distil the impact of key factors on the nature and extent of training. 
Table 12.

\section{Conceptually Clustered Matrix of Factors}

\begin{tabular}{|c|c|c|c|c|}
\hline S.No. & Main factors and attributes & Intensity & Respondents & Researcher's notes \\
\hline 1 & $\begin{array}{l}\text { Workplace change } \\
\text {-structural } \\
\text {-ownership } \\
\text {-technological }\end{array}$ & $\begin{array}{l}\mathrm{H} \\
\mathrm{H} \\
\mathrm{H}\end{array}$ & $\begin{array}{l}2,3,4,5 \\
2,3,4,5 \\
2,3,4,5\end{array}$ & New technology, platformisation, and strong work culture. \\
\hline 2 & $\begin{array}{l}\text { Market orientation (M0) } \\
\text {-information sensing } \\
\text {-information dissemination } \\
\text {-organisational response }\end{array}$ & $\begin{array}{l}\mathrm{M} \\
\mathrm{M}-\mathrm{H} \\
\mathrm{M}-\mathrm{L}\end{array}$ & $\begin{array}{l}2,3,4 \\
2,3,4 \\
2,4,5\end{array}$ & $\begin{array}{l}\text { Collaborative product codevelopment and design determines } \\
\text { the response. }\end{array}$ \\
\hline 3 & $\begin{array}{l}\text { Learning orientation (L0) } \\
\text {-commitment to learning } \\
\text {-open-mindedness } \\
\text {-shared organisational vision }\end{array}$ & $\begin{array}{l}\mathrm{H} \\
\mathrm{H} \\
\mathrm{H}\end{array}$ & $\begin{array}{l}1,2,3,4,5,6 \\
2,3,4 \\
2,3,4,5\end{array}$ & $\begin{array}{l}\text { Evidence of innovation and critical thinking is evident- } \\
\text { dependent on the nature of codevelopment. }\end{array}$ \\
\hline 4 & $\begin{array}{l}\text { Quality management systems (QMS) } \\
\text {-Commitment \& sharing information } \\
\text {-continuous improvement } \\
\text {-teamwork }\end{array}$ & $\begin{array}{l}\mathrm{H} \\
\mathrm{H} \\
\mathrm{H}\end{array}$ & $\begin{array}{l}1,2,3,4,5,6 \\
2,3,4,6 \\
1,2,4,5,6\end{array}$ & $\begin{array}{l}\text { No formal accreditation standards but highly customised } \\
\text { internal quality standards exist. }\end{array}$ \\
\hline 5 & Employee turnover & M-L & $2,3,5$ & Lower than industry average. Limited impact. \\
\hline 6 & $\begin{array}{l}\text { Enterprise size, complexity and } \\
\text { ownership }\end{array}$ & $\begin{array}{l}\mathrm{H}, \mathrm{H} \text { and } \\
\text { MNC }\end{array}$ & $1-5$ & $\begin{array}{l}\text { Large and complex organisation; US ownership—-strong } \\
\text { culture. Diverse and complex portfolio. }\end{array}$ \\
\hline 7 & Temporal dimension & M-L & $2,3,4,6$ & $\begin{array}{l}\text { Depends on the nature of collaborative codevelopment } \\
\text { activity. }\end{array}$ \\
\hline 8 & Geographical dimension & $\mathrm{L}$ & $1,2,3,4$ & $\begin{array}{l}\text { Minimal training impact on work from different } \\
\text { geographical areas. }\end{array}$ \\
\hline 9 & $\begin{array}{l}\text { Strategic HRM approach and workforce } \\
\text { composition } \\
\text {-rewards \& performance management systems } \\
\text {-career planning } \\
\text {-recruitment skills level \& operating roles; and } \\
\text {-use of non-standard employment }\end{array}$ & $\begin{array}{l}\mathrm{H} \\
\mathrm{M}-\mathrm{H} \\
\mathrm{M} \\
\mathrm{L}\end{array}$ & $\begin{array}{l}2,3 \\
2,3,4 \\
1,2,3 \\
1 \text { and } 3\end{array}$ & $\begin{array}{l}\text { Current reliance on experienced employees is gradually } \\
\text { reducing as it develops a site-specific hierarchy and local } \\
\text { learning and development capabilities. }\end{array}$ \\
\hline
\end{tabular}

Legend: $H$ =High; $M=$ Medium; $L=$ Low; $N=$ Nil

\section{Extent of Training}

Organisation $G$ has a 2-4 week integration programme and a mandated training of 80 hours per person, per annum. The training volume is significantly influenced by Organisation G's US ownership and its ethnocentric approach in its people management and business processes. Specifically, Organisation G's culture, technological complexity, 
quality management systems, strong LO, and workplace change influence training volume. Employee turnover has a lesser impact on training volume.

\section{Nature of Training}

As noted earlier, there are four areas of investment in training, most of which are technical in nature and have a strong component of group- or firm-specific knowledge. Even its generic career development-related training, offered in Organisation $G$ University's course content, has a high degree of contextualisation and customisation to meet Organisation G-specific business requirements. Organisation G has highly customised and Organisation G-specific processes and standards of development, which, it believes, cannot be easily transferable to other organisations in a similar industry sector, suggesting a resource-based view of the firm (Barney, 1991). Because of Organisation G's evolving training delivery capabilities, there is a significant reliance on external training providers for delivery. Organisation G's quality management processes and standards require formalised approaches for the definition, measurement, and evaluation of training.

\section{Discussion}

What most shapes the nature and extent of training is Organisation G's market leadership. Being a market leader for a long period of time has enabled it to develop a huge training and development infrastructure and employ a significant percentage of RCGs in its workforce, which helps it to keep its costs down. While the percentage of RCGs is still low $(20-25 \%)$ in India compared to the US (60-70\%), the mandate for hiring now is to recruit at least $30 \%$ of the RCGs in each year's hiring plan. Secondly, although a significant proportion of Organisation G's current recruitment focuses on the hiring of experienced employees, there is still a strong need to put them through the integration programme so that they learn the Organisation G-specific work culture, processes and standards.

Organisation G's high LO, strong internal quality standards and benchmarks for development (proxy for formal quality management accreditations or a formal TQM philosophy), and medium-to-low MO create an environment conducive to product development and technological innovation. While high LO supports double-loop 
learning, its QMS helps to effect planned continuous improvement and, its low to medium MO enables selective information sensing and dissemination, owing primarily to its product superiority and market leader mindset. However, this is changing. A focus on platformisation will require engaging in higher level learning and developing stronger MO abilities.

As Organisation $\mathrm{G}$ establishes new standards of performance and development to support its platformisation structure and work flow, new learning and development needs would need to be embedded in its routines. Industry pundits see the move to platformisation by Organisation $\mathrm{G}$ as a strategy to expand its market share by developing an integrated motherboard for a variety of computing and communication devices. This move would require development of strong market intelligence and client-specific capabilities and is likely to create disequilibrium in the semiconductor and microprocessor market. Consequently, such a move is expected to further the expansion and development of new technology and client management capabilities.

\section{Conclusion}

This case demonstrated that even a large market leader such as Organisation G, with a highly developed corporate university infrastructure, could not successfully exploit its well developed learning infrastructure in Indian settings. It needed to build up operations in India by initially recruiting a pool of experienced personnel and then to gradually introduce its global strategy of significant RCG hiring. The case study also points to the inadequacies in the Indian engineering curriculum, the scarcity of semiconductor resources in the external labour market, and the partial success of its highly ethnocentric approach due to an evolving site-specific integration and post-integration programme. Unless the organisation realigns its learning and development to the Indian sites' unique needs, its ability to ramp up operations using RCGs will be fairly limited, and growth through lateral hiring, although possible, remains an expensive option. 


\title{
CHAPTER 5.8
}

\section{ORGANISATION H}

\begin{abstract}
About Organisation $\mathbf{H}$
Organisation H is a medium-sized Indian owned ITSS organisation. Established in 1994 by the investment arm of a large Indian multinational group, Organisation H currently employs about 800 employees at its three software development centres in India. Headquartered in Bangalore, India, Organisation H caters to the financial services, health, energy, technology, manufacturing, and public services industry sectors. It has onsite development support staff and sales and marketing offices in the US, Europe, Middle East and Asia Pacific. Organisation H offers business, technology, and quality consulting services and solutions in the following six areas:
\end{abstract}

- Application management services

- Value added services

- Customer relationship management (CRM)

- Enterprise solutions (e.g. SAP)

- Product services

- Web services

Figure 22 provides a snapshot of its services portfolio. Most (95\%) of its workforce is employed in operations- $90 \%$ IT in services projects, $5 \%$ in product development, and the remaining (5\%) workforce is in enabling functions such as HR, sales and marketing, quality, finance, and administration. Most (80\%) of its business is from repeat clients, most of whom are based in the US. Organisation $\mathrm{H}$ has strategic tie-ups with two large US ITSS firms for developing expertise in certain technologies and partnering with them on certain projects.

About $90 \%$ of its workforce consists of full-time permanent employees, the remaining $10 \%$ are contract workers employed through software vendors for very specific skill sets that are either not readily available from external labour market or the nature of the project is short-term and does not justify ongoing employment. 
Figure 22: Organisation H: services portfolio

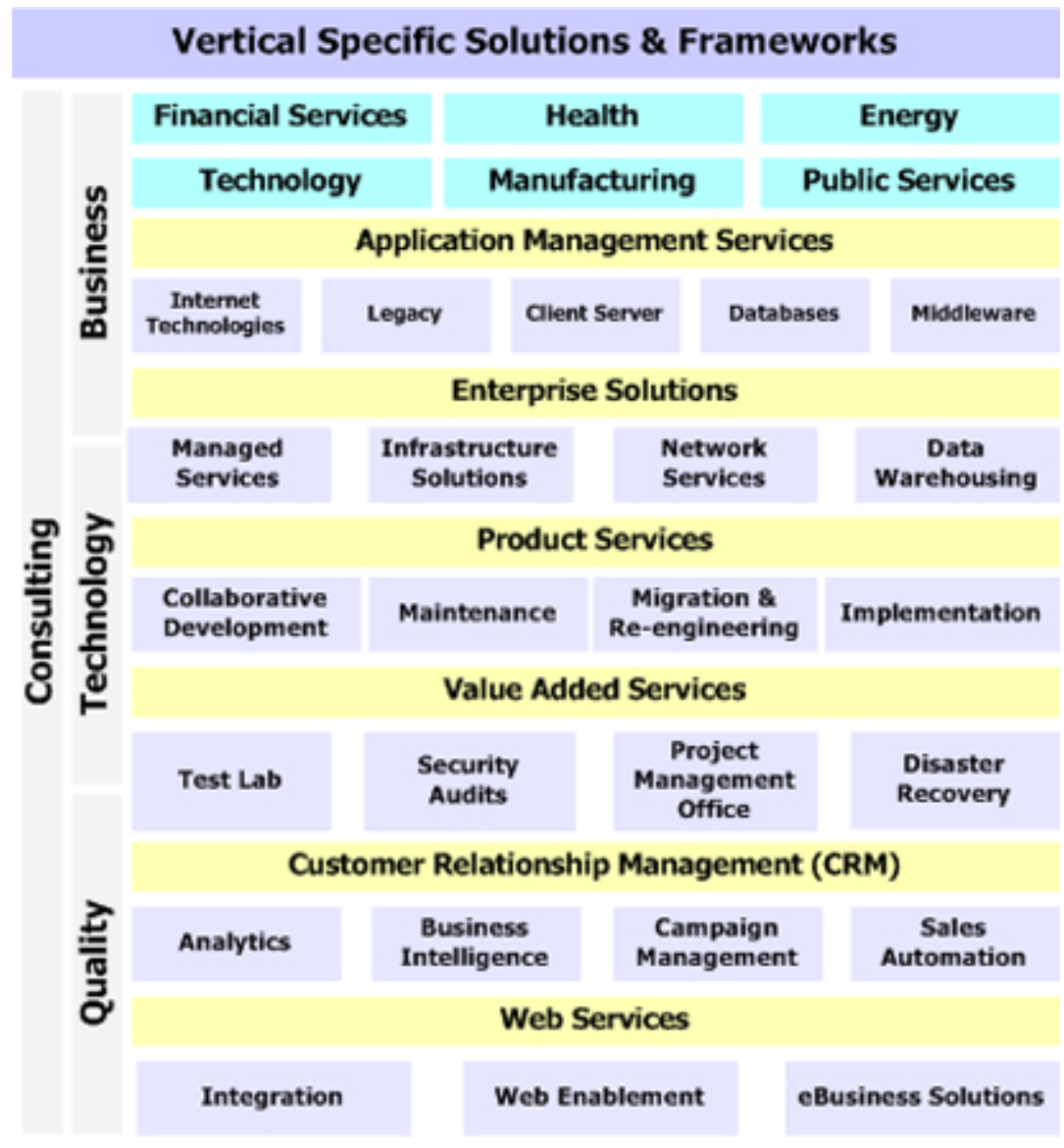

\section{Organisation Structure and Processes}

The organisation is structured around industry sectors to better utilise its domain knowledge and client-specific capabilities for delivering its services and solutions. Organisation $\mathrm{H}$ follows a Client-Driven Engagement Model where it offers flexible modes of client engagement such as onsite, offshore, dedicated ODC, joint venture model, built-operate-transfer and other hybrid forms of client engagement. Depending on the client needs and their risk tolerance levels, and Organisation H's need to use clients' skills and capabilities, different forms of engagement are agreed.

Based on the nature of projects received by the organisation and the client engagement models adopted, it attempts to maintain a pyramid structure. In other words, apart from the flexible modes of engagement it offers to its clients, the focus is also on managing employee costs by recruiting an appropriate mix of recent college graduates and laterals. 


\section{Business Processes, Strategy, and Work Organisation}

Organisation H's sales and marketing team responds to requests for proposal and information from prospective clients. Based on its strengths and capabilities it advances negotiations with existing and new clients. The focus is more on expanding in existing domains where it has already developed some expertise and capabilities. This growth strategy has been successful as Organisation $\mathrm{H}$ continues to secure most of its revenues as repeat business from its existing client base and areas of specialisation.

Within each industry sector (see Figure 22), work is further divided and organised into six main technology areas:

- Mainframe technologies (e.g. IBM and AS 400)

- Client server technologies (e.g. JAVA, C++, visual basic, D2K)

- Software skills (e.g. VM/ESA, COBOL, SQL/400, MS.NET)

- Operating systems (e.g. Unix, Linux, Solaris, Windows, Mac OS)

- Relational database management systems (e.g. Oracle, SQL, DB2, Sybase)

- E-commerce (e.g. ERP(e.g. SAP and PeopleSoft), CRM, SCM)

The present case focuses on two project groups under application management services: one having a wider programme management role_covering a range of client accounts, and the other, servicing a small application development and maintenance project in the financial services industry sector.

The above strategy of verticalisation is akin to the notion of flexible specialisation (Piore \& Sabel, 1984), wherein organisations develop competencies or specialisation in a number of areas to fully exploit market opportunities as these unfold, thus suggesting a slightly differentiated services market (Boxall, 2003). Although Organisation H's initial focus was on financial services industry, but later it expanded to life sciences, manufacturing and energy sectors. One programme manager summarised Organisation H's approach to exploring new business:

As you notice our organisation "Organisation H" is a "services company", so we have no limitations to tell our clients that we do this ....We don't do this kind of a thing. We tell them we do everything under the sun. 
Such an approach allows gradual growth in different industry areas where, although it does not have domain expertise, it has the technical expertise to deliver solutions. Organisation $\mathrm{H}$ develops its domain knowledge mostly through learning provided by its new clients, a view consistent with Banerjee's $(2004,2006)$ findings. The Project Manager-Application Development and Maintenance added:

You need a subject matter expert who knows about that domain. We are a technology company. OK. So, now, few clients say, you guys should have your own subject matter experts with you, but in our case, most of the time it's the client who provides it, OK.

Nevertheless, dynamic coordination between client and service provider requires some common ground. An HR manager added:

\footnotetext{
It's not just the client... what the client is asking us... but we have to look at our skills and our perspective. ... But even our expansion should happen around the areas which we know. We can't take a risk for which we don't know.
}

\section{Nature and Extent of Training}

Most $(85 \%)$ of its training is technical in nature and is in six technical areas noted earlier and, includes quality management training. The organisation offers about 4-6 weeks of induction training to new hires in a range of technology and project-specific areas. The nature and extent of such training varies with its annual skills forecast and the nature of projects in the pipeline. Lateral hires (people with more than 2-3 years of experience recruited from the external labour market) undergo a 2-week induction programme. The nature of behavioural and other generic training focuses on people management, communication skills, and some management development programmes.

Apart from training staff on quality management, which is almost entirely sourced internally, the organisation relies on external training vendors for most of its behavioural and technical training. A competitive screening and bidding process for external training vendors is followed. Although HR recruits them, it is for the project managers to appraise the content and award the contracts to various training providers.

Induction training is followed by project-specific training, most of which is highly technical in nature and focuses on understanding clients' domain and business logic. 
Clients' domain and business process training is usually delivered by the clients and is included in the project cost. Even in cases where there is domain specific expertise available, most solutions require a contextual understanding of the clients' standard business practices.

There is limited evidence of investment in career development of staff at Organisation $\mathrm{H}$. Organisation $\mathrm{H}$ does not support or prepare employees to up skill for assuming higher responsibilities by enrolling, for example, in specialist technology courses or higher education programmes. The focus currently is on enhancing project and role-specific skill sets. Figure 23 provides an overview of temporal dimension of training.

\begin{tabular}{|llll|}
\hline $\begin{array}{c}\text { Induction } \\
\text { training }\end{array}$ & $\begin{array}{c}\text { Project -specific } \\
\text { training }\end{array}$ & $\begin{array}{l}\text { Management } \\
\text { \& leadership } \\
\text { training }\end{array}$ & $\begin{array}{c}\text { Domain-specific } \\
\text { training }\end{array}$ \\
\hline $\begin{array}{l}\text { 2-6 weeks } \\
\text { Role } \\
\text { dependent }\end{array}$ & $\begin{array}{c}\text { 2-3 weeks } \\
\text { Project } \\
\text { dependent }\end{array}$ & Role dependent & $\begin{array}{c}\text { Project \& industry } \\
\text { dependent }\end{array}$ \\
\hline (TIMELINE DEPENDENT ON THE NATURE OF PROJECT AND BUSINESS \\
GROUP NEEDS)
\end{tabular}

Figure 23. Temporal dimension of training: Changing emphases.

The focus of Organisation H's HR department in relation to training is more of a logistic support function. Training responsibility is devolved to the project and quality managers and, except for quality management training, delivery is mostly sourced externally. Being a Level 5 CMM certified organisation, it provides mandated quality management training for every new employee. The focus of such training is to understand how Organisation H's quality management processes and standards are set and implemented. Additionally, for maintaining CMM Level 5 certification, the organisation has to ensure it allows at least 40 hours of training per person per annum spread between technical and behavioural training. 


\section{Analysis of Factors Influencing Provision of Training}

\section{Workplace Change}

Organisation $\mathrm{H}$ has undergone reorganisation following its acquisition and merger with another small software development organisation. The reorganisation did not result in any major changes as there wasn't any scope for integration in the core areas. The merger was more structural in nature and had some overlaps in the enabling functions. However, as each organisation had their own training systems, the impact on any additional training demand or diversity was not noted. Changes in technology and its emergent strategy of "not saying no" to new projects, in areas where it does not have sufficient capabilities creates opportunities for further investments in training and links in with Banerjee's (2004) notion of dynamic coordination. Such coordination may not always be successful.

\section{Quality Management Systems (QMS)}

Organisation $\mathrm{H}$ is an ISO 9000 and CMM (Level 5) accredited organisation. The accreditations suggest high levels of process maturity and predictable levels of software development processes and service delivery. Further, Organisation H's centralised quality assurance group is considered as an extended part of its service delivery. The quality management group defines all the business and software development processes to bring about benefits of standardisation, reduced errors, and consequently reduced costs and resource requirements.

Organisation H's explanation for investment in quality is to enhance its market signalling, cost efficiencies and predictable service quality for its clients, by using repeatable and predictable software development processes. On a closer analysis of its quality management systems, although Organisation $\mathrm{H}$ has well developed benchmarks and standards of performance, its ability to disseminate these standards and best-practice information is a real challenge. Consequently, it also affects its LO and MO abilities. A quality manager noted: "So, the biggest challenge is to share the knowledge. Unless you do that, and unless that happens, there is no improvement... you cannot keep growing."

Although the tools are available, dissemination is limiting the development of a strong quality culture. Additionally, its excessive compliance focus (for CMM and ISO 
accreditation) has resulted in limited continuous improvement efforts. A project manager explained:

So, there would be definitely bare minimum quality that you have...to follow, to stay as a CMM certified company. And, there are few improvement programmes depending upon project pressures and what kind of time lines and that is little lenient. I mean not every project needs to show improvement in that process area — they [are] already tight scheduled. But definitely, the basic guidelines have to be followed like the metrics collection has to happen once in a month. That has to be sent to the quality centre head, so the quality centre head would receive the metrics... in let's say the number of errors ....if there are some deviations, some root cause analysis and all this will be done.

\section{Market Orientation}

Although Organisation H's Client-Driven Engagement Model follows pre-defined quality management benchmarks and metrics, it also allows for customisation of projects to cater to the clients' unique engagement needs. Most of the information sensing begins with the requests for proposal (RFP) documents sent to its prospective clients. While RFPs are generic documents for prospective clients, further interest results in sales presentations and demonstrating Organisation H's service delivery capabilities. Initial business requirements specification is undertaken by a team of sales and technical staff. Organisation H's QMS enable the development of service delivery metrics for clients' specific requirements. As noted in one of the organisational service documents:

\footnotetext{
Our solutions are tailored around the unique CDEM (Client Driven Engagement Model). We quickly develop a deep understanding on the client's distinctive needs, and then we deploy our strong resource base and processes to deliver measurable business value. This model works at the core of every project laying down very clear responsibilities in the organization for a particular engagement, thereby helping to shrink delivery cycles and cut down on defects.
}

Higher and lower level solutions design, development of service level agreements and, information dissemination follows. The project teams in India are informed concurrently. Based on the internal capabilities and the resources available, training needs are identified and, operational and training responses are framed. Organisation H's MO is influenced by its quality management capabilities. A quality manager explained:

So at pre-sales stage itself, we have all the other quality aspects - in terms of the processes we have... How do we exactly propose? And, how we get to know what the requirement of 
the client is and how do we showcase our strengths? .... So, it is nothing you define [and] keep it forever. So, you have many dynamics. Pitching within the course of your various projects... that is where your process improvement comes in. So, to us as an equation it starts off as early as pre-sales and once you get your proposal.

Overall, Organisation $\mathrm{H}$ has medium to high levels of MO. Information dissemination is not fully developed.

\section{Learning Orientation}

Organisation $\mathrm{H}$ has medium levels of LO. Its commitment to learning is medium to low and is evident in its limited provision of training and its training and development infrastructure, focusing more on meeting project specific needs. Organisation H's ability to develop a shared vision or knowledge between business groups is fairly limited. There is only one technology forum and its intranet site for sharing information is not yet launched. One programme manager added:

...the bulletin board is not active and our intranet is not up and running. Otherwise, yes, there is a forum that when somebody has a problem, put it all on the forum and anybody in the company is free to answer .... We are working on a technology called shared forum, we exclusively put up a group on the web, on the internet, and we were sharing our thoughts..., so this keeps happening within the organisation and outside this organisation as well.

Limited evidence was noted in the organisation's ability to critically challenge clients' assumptions or their own business processes. The focus is more on adhering to their best practice guidelines and standards established by the quality management team. However, even adhering to its quality management standards is sometimes problematic as the information sharing and dissemination systems are not well developed. The metrics reside centrally within the quality management group. A quality manager stated:

So you send in the request to the quality assurance group (QAG) and they will debate on whether to approve or disapprove, or, have more discussions and clearly understand why it is required etc, etc. And, we have that database also, how was it a standard deviation, or, it could be clients demand, so we have all the records of all the processes step by step, which are required probably for the execution of a particular type of project. 


\section{Employee Turnover}

Organisation $\mathrm{H}$ has medium to low employee turnover ranging between $9-12 \%$. The bulk of the turnover is at the middle to senior management level, which does not directly result in training needs as these roles are mostly filled by hiring experienced people. Overall, employee turnover has a medium to low impact on training volume and diversity. Employees quit mostly for lack of career development and opportunities for enhancing new technology skills. To overcome this, where possible, Organisation H's business development and project managers encourage clients to undertake software development in new technology areas or gradually phase out the legacy technologies with new ones. Such coordination benefits both the service provider (through better billing rates and employee retention) and the client (by using new technology and advanced functionalities). The above view is consistent with Banerjee's (2004) description of dynamic coordination between the client and service provider. Depending on which technology the client wants to work on (legacy, non-legacy or emerging technologies), the impact on the provision of training would vary accordingly. Typically, legacy and emerging technologies require more investment in training than a current technology area.

\section{Enterprise Size, Complexity, and Ownership}

Instead of mere enterprise size, Organisation H's growth in diverse industry verticals and service lines has added to the project and technology complexity. Consequently, this has increased the demand and diversity of training. However, the relationship between project complexity and training is not straightforward. This relationship is influenced by the organisation's "make" or "buy" strategy. The organisation has a leaning towards the "buy" decision, which reduced the extent to which such training needs to be provided.

Further, its learning needs are also met by its clients, especially in developing domain knowledge expertise and understanding clients' proprietary software tools and applications. Specialist skills are sourced externally from a range of software vendors with whom it has strategic partnerships. In terms of ownership, it appears from the case analysis that the Indian promoters - an investment arm of a large investment firm, consider this venture as another "investment" in their portfolio of investments. The shortterm focus thus is on enhancing its return on investment for its shareholders. 


\section{Temporal Dimension of Projects}

Organisation $\mathrm{H}$ requires high training volumes at the beginning of a new client project. The learning is steep for the project team when project specifications are finalised and clients' business logic and their domain knowledge needs to be understood. Gradually, as the team begins development, their learning needs decline over a period of time (Bartel \& Lichtenberg, 1987). The nature of project in software development and technology lifecycle also determines the volume and diversity of training. As noted earlier, maintenance projects, which are typically using legacy technologies, require higher volume and diversity of training, as against non-legacy systems. Similarly, in application development projects, legacy and emerging technologies attract higher investments in training as compared to non-legacy systems. In a software services environment, project enhancements or change requests do not significantly impact the volume of training, as the technology is usually the same, only certain parameters or features of the application may change, which does not require any further up skilling.

\section{Geographical Dimension}

Technology cuts across borders and there aren't any substantive differences in training for a particular technology, Organisation H reported that ITSS firms in the US are open to the use of emerging technologies and innovations, as compared to the more conservative European markets, where a lot of projects-development or maintenance use legacy technology systems. The latter requires higher levels of training because there are limited numbers of employees in the external labour market with skill sets are mostly obsolete. This is true as IT professionals do not want to be seen as working on old technologies.

\section{Strategic HRM Approach, Skills Level, and Workforce Composition}

Organisation $\mathrm{H}$ shows limited evidence of a strategic approach to its people management practices. Its performance management systems occur annually and are not integrated with quality or other management standards. Organisation $\mathrm{H}$ does not offer any formal career development opportunities for staff. The closest career development it offers is to train employees in new technologies if such a new technology project comes on board. Although the organisation has a mandate to "maintain a pyramid" to contain its people costs, there is still a significant level (70-80\%) of lateral hiring (experienced employees) 
that takes place, thus suggesting the "buy" versus "make" decision. Nevertheless, its graduate hiring does require some further up skilling. As noted by a quality manager:

\begin{abstract}
Why training is triggered? This could be important to you. We not only hire IT engineers, we also hire non-IT engineers, like a mechanical engineer. So, when we have 20 or 40 hires (freshers), if there are people who haven't even got exposed to IT ...so we don't separate them out, we put all these 40 guys irrespective of what technologies they are in [to] start training from what software is? What they need to do. ... Today, [there is a] gap still between an academic guy and what you do, so [training] kind of fills that gap.
\end{abstract}

Apart from the gaps in the curriculum and costs rationale for recruiting albeit a small percentage of college graduates, its Manager- Quality added:

\begin{abstract}
Yes, one reason is the supply and the demand. And the second reason is it's been a practice in the industry. And it is also seen that people with non-IT kind of exposure perform excellent because of their energy levels they are charged up - they want to learn something new. ... See, the fresher life span in a company, you cannot expect them to stay more than two years with you, so what ever you can get from them is within those two years. After that, again you get a pyramid. ...you also need people to think, to analyse, do problem solving, this comes with any other engineering stream as well and today most of the engineering streams are not ignorant on the technology.
\end{abstract}

\title{
Specific Impact of Factors on Nature and Extent of Training
}

The following section provides an analysis of how various factors affect the nature (externalisation, diversity, transferability, and formalisation) and extent (volume) of training. Following Miles and Huberman (1994), a conceptually clustered matrix was first developed.

\section{Extent of Training}

Organisation $\mathrm{H}$ offers a 2-6 week induction programme and another 2-3 week project specific training. Additionally, a mandated training for 40 hours per person, per annum is also offered. The training volume is significantly influenced by Organisation H's ownership, which has a short-term focus, and thus its 'buy' HR strategy. Training volume is also influenced by technological complexity, quality management systems, and to a lesser extent by employee turnover and geographical dimension of training. 
Table 13.

\section{Conceptually Clustered Matrix of Factors}

\begin{tabular}{|c|c|c|c|c|}
\hline S.No. & Main factors and attributes & Intensity & Respondents & Researcher's notes \\
\hline 1 & $\begin{array}{l}\text { Workplace change } \\
\text {-structural } \\
\text {-ownership } \\
\text {-technological }\end{array}$ & $\begin{array}{l}\text { M } \\
M \\
H\end{array}$ & $\begin{array}{l}1,2,4,5 \\
1,2,4 \\
3,4\end{array}$ & A change to new technology is a key factor. \\
\hline 2 & $\begin{array}{l}\text { Market orientation (M0) } \\
\text {-information sensing } \\
\text {-information dissemination } \\
\text {-organisational response }\end{array}$ & $\begin{array}{l}\mathrm{H} \\
\mathrm{M}-\mathrm{L} \\
\mathrm{M}-\mathrm{H}\end{array}$ & $\begin{array}{l}3,4,5 \\
3,4,5 \\
3,4,5\end{array}$ & $\begin{array}{l}\text { Client-driven engagement model allows for a networked } \\
\text { approach to learning. Information dissemination is an issue. }\end{array}$ \\
\hline 3 & $\begin{array}{l}\text { Learning orientation (L0) } \\
\text {-commitment to learning } \\
\text {-open-mindedness } \\
\text {-shared organisational vision }\end{array}$ & $\begin{array}{l}\text { M-L } \\
\text { M-L } \\
\text { M-L }\end{array}$ & $\begin{array}{l}1,2,3,4 \\
1,23,4 \\
1,3,4\end{array}$ & $\begin{array}{l}\text { Limited evidence of innovation and critical thinking- } \\
\text { partly explained by the nature of work received and the low } \\
\text { level of information sharing abilities. }\end{array}$ \\
\hline 4 & $\begin{array}{l}\text { Quality management systems (QMS) } \\
\text {-Commitment \& sharing information } \\
\text {-continuous improvement } \\
\text {-teamwork }\end{array}$ & $\begin{array}{l}\mathrm{M} \\
\mathrm{M}-\mathrm{H} \\
\mathrm{H}\end{array}$ & $\begin{array}{l}1,2,3,4 \\
1,2,3,4 \\
1,2,3,4\end{array}$ & $\begin{array}{l}\text { Although metrics and benchmarks or software development } \\
\text { processes exist, sharing these metrics within and across } \\
\text { projects is a challenge. }\end{array}$ \\
\hline 5 & Employee turnover & $\mathrm{M}-\mathrm{L}$ & $1-4$ & Lower than industry average. Limited impact. \\
\hline 6 & $\begin{array}{l}\text { Enterprise size, complexity and } \\
\text { ownership }\end{array}$ & $\begin{array}{l}\mathrm{M}, \mathrm{M}-\mathrm{H} \text {, and } \\
\text { Indian }\end{array}$ & $3,4,5$ & $\begin{array}{l}\text { Medium-sized organisation; slightly differentiated portfolio. } \\
\text { Indian ownership and a focus on return maximisation create } \\
\text { a short-term approach to capability building. }\end{array}$ \\
\hline 7 & Temporal dimension & M-L & $3,4,5$ & Depends on the nature of technology and project. \\
\hline 8 & Geographical dimension & $M-L$ & $3,4,5$ & $\begin{array}{l}\text { Nature of work varies -legacy (Europe) versus non-legacy } \\
\text { (US) systems }\end{array}$ \\
\hline 9 & $\begin{array}{l}\text { Strategic HRM approach and workforce } \\
\text { composition } \\
\text {-rewards \& performance management systems } \\
\text {-career planning } \\
\text {-recruitment skills level \& operating roles; and } \\
\text {-use of non-standard employment }\end{array}$ & $\begin{array}{l}\text { M } \\
\text { M-L } \\
\text { M-H } \\
\text { M-L }\end{array}$ & $\begin{array}{l}1,2,4 \\
1,2,3 \\
1,2,3 \\
1,2,3,4\end{array}$ & $\begin{array}{l}\text { Current reliance on experienced employees and reliance on } \\
\text { external training providers suggest a 'buy' training decision } \\
\text { on Organisation H's part. }\end{array}$ \\
\hline
\end{tabular}

Legend: $H=$ High; $M=$ Medium; $L=L o w ; N=N i l$

\section{Nature of Training}

Organisation H's focus is on induction, project specific, domain and quality management training. Most of its training provision is technical and transferable in nature and has a strong reliance on external training providers, clients, and other software development 
firms'. Organisation $\mathrm{H}$ has high formalisation in terms of training evaluation, which is a requirement under its quality management systems. Training and development to support employees' career aspirations is minimal, as such training is considered by the organisation to be generic and transferable to other organisations and there is no learning support extended to employees wanting to pursue higher education.

\section{Discussion}

In addition to the structured induction programme, training and development at Organisation $\mathrm{H}$ is focused on supporting the project needs. Organisation $\mathrm{H}$ lacks a longterm people development focus. Although Organisation H has ISO 9000 and CMM (Level 5) accreditations and well developed internal benchmarks and processes for software development, its ability to disseminate and share this knowledge is an issue and is affecting its ability to maximise the benefits from its internal standards of performance and best-practice processes templates.

Owing to the nature of work and the centralised evaluation of project specifications by its quality management team, Organisation H's project teams are unable to develop a shared vision and demonstrate high open-mindedness-knowledge questioning values (essential for strong LO) for its existing business processes and clients' project specifications.

Overall, Organisation $\mathrm{H}$ has medium to high QMS and MO medium to low LO. Organisation H's inability to share, disseminate, and challenge key process knowledge and metrics, have resulted in medium to low LO and medium to high MO. The above approach to learning suggests Organisation $\mathrm{H}$ is engaging in incremental and adaptive or single-loop learning (Argyris \& Schon, 1978; Baker \& Sinkula, 2002). Further development of domain specific expertise is needed in addition to what is currently learnt from its clients. Such knowledge needs to be shared within the industry-specific groups for deeper learning and framing better solutions. This requires a culture of sharing and freeing up of resources to enable it.

\section{Conclusion}

This case provided insights about how training is organised in a medium sized, Indian owned, ITSS organisation. Typically, the reliance is more on the "buy" decision by 
acquiring skills from the external labour market. Where the organisation has decided to invest in training for recent college graduates, the training design and delivery are outsourced to numerous external training providers. Organisation H's limited commitment to expand its learning and development infrastructure is also evident in the limited career development opportunities it offers to its employees.

This case study confirms the gaps in the educational curriculum relevant to the IT sector and also reinforces that such gaps in the curriculum can be very easily overcome by providing focused training in areas where a firm offers its services. However, for the organisation to be able to plug the gap, it is faced with a typical "make" or "buy" skills development decision. It follows from the analysis that a "make" decision requires top management commitment and sustained growth rates, critical for justifying training investments. The "buy" decision is more typical of organisations in the early stages of their growth lifecycle and those that are developing their organisational capabilities. 


\title{
CHAPTER 5.9
}

\section{ORGANISATION I}

\begin{abstract}
About Organisation I
Organisation I is a small, offshore software product development centre, wholly owned by a small US-based software product development organisation. Based in Hyderabad, India, Organisation I provides a range of software codevelopment support for its US parent's software product used in hospital operation theatres. The Indian offshore software development centre (ODC) was established in 2000 and currently employs about 150 full-time permanent staff. The software product was developed by the US team in three years (1996-1999). In 1999, the product was launched for the US markets. After about a year of the product launch, it set up the ODC in India to support its ongoing software codevelopment needs and to tap India's inexpensive IT labour market.
\end{abstract}

The ODC was initially set up to provide low-end software development services, typical of a software development product life cycle (See Figure 3, Chapter 2). Later, higher specification work was also added to the ODC's portfolio of services. Current services include testing, database administration, development and maintenance, front-end tools development, client support, and customisation services.

The software product is HIPPA-compliant, has about 30 modules, and is offered as an integrated or customised solution to 260 hospitals in the US and Canada. The product architecture provides solutions in following areas:

- Pre-operative information solutions: Scheduling of administrative roles such as hospital admission, discharge, follow-ups, and pre-surgery planning for patients, anaesthesiologists, surgeons, and nurses.

- Advanced clinical solutions: Management of patient records, clinical anaesthesia, operation theatre utilisation, and tracking of routine surgical needs and specialised reports. It also has the ability to interface with a range of different hardware and software applications installed in a hospital.

- Resource management: Hospital workforce scheduling, nursing automation, patient flow, preadmission testing and so on. 


\section{Organisation Structure and Processes}

Organisation I's staff strength of 150 is spread between the following service groups: Testing (70), Oracle database management and support (30), Centura front-end solutions (20), C++ (10), Statcom client web-interface (12). The remaining employees are in enabling functions such as HR (2), finance (2), and senior management (2). Being a small ODC organisation, it has a flat management structure. Each team has a project manager, team leads or equivalent. There are very few senior management roles.

Given the multi-case embedded case study design, the following three service groups were studied in detail: Testing, Oracle and $\mathrm{C}++$. The organisation structure, for example, in the testing business unit is as follows: Project Manager-Quality Assurance (QA), Senior Manager-QA, team leaders, analysts testers, senior testers, and testers. Similarly, in the Oracle and $\mathrm{C}++$ groups, the following structure exists: Assistant General Manager, Manager, team leads, senior software engineer, and software engineers.

The majority of its workforce provides testing services for the product, using both manual and automation testing. However, recent work involves visits to client sites in the US by its $\mathrm{C}++$ and report generation teams. The Oracle team looks after the development, maintenance, and administration of databases, developing and maintaining about 140 different reports by end-users.

\section{Business Processes, Strategy, and Work Organisation}

Workflow is organised around the quarterly work targets it receives from the US. While business development and marketing teams are based in the US, Organisation I uses its Statcom client website for client updates and for providing a range of other development support. The nature of the workflow is highly structured and focuses on pre-determined tasks. There is limited interaction with the US teams. At the ODC, service groups work independently of each other. This organisation of work is mainly due to the distinct nature of the tasks performed by each work group and the nature of the skills of each group.

The business strategy of the US parent is to develop applications for the fast-growing and relatively untapped health industry sector. The ODC strategy was to tap into the 
inexpensive and skilled labour market and to exploit the time and costs arbitrage that exists in a typical Indian ODC set up. The nature of services offered typifies a slightly differentiated service market (Boxall, 2003). Being a product organisation, setting up an ODC, instead of a third-party development centre, was logical as it helps in protecting proprietary product information and ensures confidentiality. However, setting up an ODC requires strong knowledge of the host country's cultural environment and labour markets. Although its US office initially pressed for a highly ethnocentric approach in its people management practices, such as variable pay and non-standard employment, it proved to be ineffective for the Indian cultural environment and labour market conditions. Nevertheless, some operational aspects of the US such as workload planning, recruitment, and selection tests, were retained. For example, the Software Calibre and DISC (Dominance, Influence, Steadiness and Compliance) profiling tests are mandatory for selection to all technical roles, irrespective of whether the applicant is experienced or a recent graduate.

The above selection tests enabled a selection ratio of about $1 \%$, based on number of applications received and $7 \%$ based on the number of short-listed applications. These tests resulted in selecting employees with strong technical skills, and personality traits reflecting high compliance and steadiness scores and low influence and dominance scores. It also enabled most employees to be placed in specific roles requiring the performance of a narrow range of activities. While recruiting such profiles helped its people management practices, it somewhat reduced the organisation's ability to accept innovative and creative work from the US. The US rationale for adopting such an approach is premised on the low-end and less creative nature of work at the Indian ODC. Additionally, such employee profiling reduces the risks associated with any changes to the product's base programming codes.

In 2003-04, with a view to containing its wage costs, the organisation experimented with the recruitment of freshers. Limited HR success (retention) and cost effectiveness were evident, especially for the testing roles. Its Head-HR commented: "Every software engineer's dream is to be a developer". Further, the nature of the work in a product environment is considered monotonous, and the compensation structure it offered to engineer trainees was uncompetitive. Subsequently, the organisation reverted back to its dominant "buy" strategy of hiring engineering graduates with 3-4 years of experience on relevant technologies. 


\section{Nature and Extent of Training}

The nature and extent of training provided is mostly (more than $80 \%$ ) technical and product-specific, and the remainder (less than 20\%) is behavioural. On average, it provides about 2 weeks of training for every new employee. The 2 -week induction programme mainly focuses on product knowledge, learning different product moduleshow the modules interact with each other and how such interaction affects specific roles for providing development support. Induction also covers the quality management standards and processes that form a part of employees' daily work routines.

Following the induction training, employees undergo functional training in their respective work groups. Such training, while mostly on-the-job, is semi-formal and is dependent on the individual's past skills and experience on a technology. For example, in the Testing group, based on past experience and skills, new employees may be required to complete both manual and automation testing.

Relative to behavioural skills training, Organisation I's investment in technical training is considered logical by the US parent because of the limited client interaction required by the ODC. Moreover, the need for investing in soft skills is further reduced by its focused selection strategy. Overall training volume is reduced by its skills acquisition strategy, which focuses on "buying" skilled and experienced personnel from the external labour market. Nevertheless, to retain and develop its few senior management employees, it invests in occasional management and leadership development programmes. Learning support for higher education and training, while negligible, is considered on a selective and job relevance basis. Few employees were allowed fee reimbursement and time for pursuing project management and Microsoft certifications. Overall, employees are encouraged to learn new technologies such as Microsoft .Net in their free time.

The organisation's ISO 9001-02 certification organisation requires a high degree of formalisation for delivering its quality management and product training. Training on ISO-driven processes is externally provided. HIPPA certification and compliance also requires high formalisation of training. However, for other functional training, formalisation of training is low and mostly provided on-the-job, using internal subject matter experts. While most product-related training is provided internally, there is limited 
formal "Train the Trainer" training. Nevertheless, some pre-developed resources are used for developing trainers' skills. Its Assistant General Manager Oracle Group noted:

So I will bring the copy of 50 topics, give it to them and tell them these are people who know this, who have knowledge in this area. So I will suggest some names. For these topics you contact these persons. He is very familiar with that, but with other topics contact anyone in the team. This kind of idea will be given and timing ... I cannot fix the timing. They will be busy with some deliverable or some deadlines. Go to the individual, talk to them, fix the time with him and whenever he is free he will spend some time with you.

Client requests for product customisation often impact on the training needs of certain groups. These include the testers, crystal reports team, and $\mathrm{C}++$. At a group level, training provision is informal and often spread over time.

\begin{tabular}{|c|c|c|c|}
\hline $\begin{array}{l}\text { New hire } \\
\text { training } \\
\text { (Product knowledge } \\
\text { \& quality) }\end{array}$ & $\begin{array}{l}\text { Job/Role- } \\
\text { specific } \\
\text { training }\end{array}$ & $\begin{array}{l}\text { Refresher } \\
\text { training }\end{array}$ & $\begin{array}{l}\text { New technology } \\
\text { \& leadership } \\
\text { development }\end{array}$ \\
\hline 2 weeks & $\begin{array}{l}1 \text { week (group } \\
\text { dependent) }\end{array}$ & $\begin{array}{l}\text { Product } \\
\text { enhancements } \\
\text { ent dependent) }\end{array}$ & $\begin{array}{l}\text { Programme } \\
\text { dependent }\end{array}$ \\
\hline \multicolumn{4}{|c|}{ (TIMELINE DEPENDENT ON ROLE AND EXPERIENCE OF EMPLOYEES) } \\
\hline $\begin{array}{l}\text { 2005-2006 Key Focus } \\
\text { Induction \& } \\
\text { product training }\end{array}$ & $\begin{array}{l}\text { as of Training: } \\
\text { Job/Role-specific } \\
\text { training }\end{array}$ & $\begin{array}{l}\text { Refresher } \\
\text { training }\end{array}$ & $\begin{array}{l}\text { Developing senior } \\
\text { nanagers \& new } \\
\text { technology capabilities }\end{array}$ \\
\hline
\end{tabular}

Figure 24. Temporal dimension of training: Changing emphases.

\section{Analysis of Factors Influencing Provision of Training}

\section{Workplace Change}

The organisation has witnessed two major workplace changes since 2001. First, when demand for testing was high, it employed 40 graduate trainees for testing roles. Recruiting freshers as against experienced employees resulted in high training demand. Second, it switched to web-based platform and solutions for the product. It moved to Microsoft's .Net technology. The move proved not to be successful for a range of operational and capability reasons. Nevertheless, it resulted in the development of new technology skills. In both cases, its technological and structural changes resulted in 
increased skill development.

The above changes were closely intertwined with its recruitment, client satisfaction, and market share enhancement strategies. According to its head of India operations, considering the external labour market for certain skills, it needs to develop staff skills on new technologies to retain them and to satisfy clients' needs. The high rate of technological obsolescence reduces the talent pool for certain legacy systems from the labour market, thus providing an opportunity for the organisation to move to the next level of technology, and consequently be able to tap into a new pool of skill sets.

\section{Quality Management Systems (QMS)}

Being ISO-certified, Organisation I is a highly process-driven organisation. Although it does not have a dedicated quality management group, it considers its quality processes to be comparable to level 3 of CMM. Further, being a product organisation, investment in quality accreditations is not high on its agenda. The head of HR commented:

\footnotetext{
We never believed in going for certification because none of our customers insisted on it. That way, Microsoft and IBM are not CMM-certified. Unknown Indian companies are crazy for CMM Level 5, and they want to tell the outside world that they can do it.
}

Nevertheless, quality plays a critical role, as service deliverables and billing rates for the Indian ODC are tied to the quality of its service. Failure to adhere to client specifications in terms of deadlines, or the discovery of bugs in the application, attracts severe monetary penalties and loss in revenues. ISO standards and processes are mapped for each stage of the standard waterfall model of the software development lifecycle. Thus, investment in ISO processes requires software development processes to proceed in a certain way. This process-driven approach also results in formalisation of its quality and product training.

Training volume is also influenced because the majority of work at ODC is in the testing area $(46 \%)$, which requires training in a narrow range of quality tools for a large group of testers. In the absence of a dedicated quality management group, training on quality tools and processes is provided by external vendors. Consequently, its focus is more on the internal dimension of quality (costs reduction and information sharing). A limited impact is noted on clients' SLA development owing to the nature of the development process. 


\section{Market Orientation}

Being a captive product-environment ODC, its $\mathrm{MO}$ is medium and distributed between the US and Indian facilities. Its mindset of selling a superior product often comes at the cost of not meeting all its client needs. High adaptive learning, often associated with high MO, would require significant and costly changes to its product architecture, which it currently does not see any need to make.

The above mindset has a negative impact on provision of training, encouraging the development of only those core technical skills necessary for its current product architecture. While the above appears to be an economically rational decision for the short-term, the organisation has started to face pressures both internally, from its employees (for moving to a new technology platform), and externally, from its clients (to customise and provide web-based solutions).

Its information sensing is done by its US business development team, and the dissemination of information and framing of responses are shared between the Indian ODC and US locations. Dissemination of information and response at the ODC are determined by the quarterly targets set in the US. In some cases, where clients request changes to product functionality, project managers from India visit clients in the US. Dissemination of clients' requested changes often results in training or new learning for ODC team members.

Consequently, the nature of work delivered by the Indian ODC is changing and the training needs of some work groups are triggered by clients' customisation requests. These changes require development of strong communication and negotiation skills for its client-facing groups and often result in provision of new learning, albeit informally, for its development teams. Such changes also affect the training needs of the US client-facing teams, whereby they receive training from the Indian ODC team on client customisation changes. A project manager commented:

How it works is, sometimes the critical functionality they will explain. Unless we see them personally we cannot understand them. So we get the idea. The directors, they visit and guide us. So here we give them [training] orally or with some documentation or whatever there might be here.... We customise it. We have our customer care, so whatever we do here we call it a release to customer care. 


\section{Learning Orientation}

ODC's LO is low considering the nature of work undertaken is low-end and in a narrow range. Further, as work specifications are determined by its US office, there is limited scope for critical thinking or innovation. In order to support delivery of its current work, its recruitment strategy focuses on people with specific software calibre and DISC personality profiles. Consequently, its training needs are reduced.

Overall, its commitment to learning is also low, as it is limited to product training. In addition, its organisation of work prevents integration between groups. Information sharing, albeit limited, occurs when a new product is released or quality management audits are undertaken. The extent to which information is shared is variable. Its senior software engineer commented:

\footnotetext{
Some groups have it. We, I don't think. DBS group has its knowledge-sharing forum. It is not a standard feature. We don't have an intranet site right now. But definitely, this is one of the agendas to maintain different group-wise forums and knowledge sharing sessions like, things to do and things not to do in different groups. Because tomorrow when we leave at least there is some person who already has the knowledge.
}

Even with new product releases, information sharing is informal and ad hoc. The above respondent further commented: "So, if [the] main product changes a little bit, it affects us. It is conveyed to us, but not formally. We have to learn that and find it out the hard way."

\section{Employee Turnover}

Despite the need to reduce employee turnover for protecting confidential product information, the organisation is confronted with a higher than industry average employee turnover of $30 \%$. High employee turnover rates have a direct impact on the volume of training. Such high levels of turnover can be attributed to low-end testing work, uncompetitive compensation structure, the monotonous nature of the work in a product environment, and night shift work. Another reason is the delay in upgrading to a new technology and a web-based platform. Technology upgrades and the changing profile of skills supply in the external labour market would require the organisation to invest in new technology training for sustainable business growth. 


\section{Enterprise Size, Complexity, and Ownership}

Small enterprise size and a single product portfolio has contained the nature of its training needs. Further, its skills acquisition strategy from the external market further reduces the need for investing in generic and technical training. Its relatively flat management structure and low client interaction also reduces the need to invest in leadership and soft skills training. Finally, because it is a wholly US-owned subsidiary, whose current strategic focus is on maximising arbitrage opportunities in India, there is limited incentive for the ODC to invest in high-end technology skills.

\section{Temporal Dimension of Projects}

Minor product enhancements have a limited impact on its total volume of training. The reasons are as follows. First, only a select employee group is affected. For example, its testing and $\mathrm{C}++$ groups receive immediate training. Second, in a codevelopment model, given that the core team and development teams are involved in the development of the new release, they are well aware of the changes they have made to the functionality. With regard to new product versions, a project manager explained:

\footnotetext{
If it is major version we need to have a lot more training. I will give you one example. Like, we support dead body interface. So to attend the training, basically, I and three more developers went to Atlanta.... So they were flying us to Europe and to Atlanta. So, like our Atlanta team and the Indian team of four persons went in for the training.... We have to spend a lot of time on this because it is not like 3 months planning, it was a 1-year plan.
}

With regard to groups not involved in the development of new functionalities, the provision of training is not uniform, because firstly, not all roles require it. Secondly, given that the information sharing is an acknowledged problem due to poor functional integration, the employees in other groups learn by trial and error, by informal and incidental ways and, often, by "figuring it out", the "hard way"'.

\section{Geographical Dimension}

There is no impact of geographical location of clients or projects on the nature and extent of training. Firstly, technology cuts across borders and, secondly, it caters only to the US and Canada. The only technological change for Canadian clients pertains to minor changes in its date and time formats. 


\section{Strategic HRM Approach, Skills Level, and Workforce Composition}

It is evident from the above analysis that the organisation has a focused skills acquisition strategy, which significantly reduces its need to invest in technology training. Further, the use of specific technical and DISC profiling test, enables it to recruit people with a narrow range of skills and a high level of experience in a given skill competency area. This approach limits job rotation opportunities and promotes working in silos. Recruiting experienced staff for permanent roles to some extent helps to reduce employee turnover and protects its product confidentiality. However, the low-end and monotonous nature of work poses a retention challenge to the organisation. Performance management is quarterly and in line with the US practices. It follows a mix of competency-based and management-by-objectives approach, which is also used for identification of training needs.

\section{Specific Impact of Factors on Nature and Extent of Training}

The following section provides an analysis of how various factors impact on the extent (volume) and nature (externalisation, diversity, and formalisation) of training. Following Miles and Huberman (1994), a conceptually clustered matrix is developed (See Table 14).

\section{Extent of Training}

On average, the organisation spends 2 weeks on training. As noted above, training volume is positively affected by product knowledge, major new product releases, employee turnover, quality management processes, and technological change. Focused skills acquisition and the cost-reduction strategy mandated by its US owners have a negative impact on the volume of training.

\section{Nature of Training}

Organisation I's diversity of training is low given the small enterprise size and single product portfolio. Moreover, its skills acquisition strategy (recruiting from the external labour market) reduces its need to provide generic and soft skills training. Training formalisation is influenced by ISO processes and the need to be assessed on certain product functionalities. As it does not have a dedicated quality management group, it relies on external training providers for training on quality processes. 
Table 14.

Conceptually Clustered Matrix of Factors

\begin{tabular}{|c|c|c|c|c|}
\hline S.No. & Main factors and attributes & Intensity & Respondents & Researcher's notes \\
\hline 1 & $\begin{array}{l}\text { Workplace change } \\
\text {-structural } \\
\text {-ownership } \\
\text {-technological }\end{array}$ & $\begin{array}{l}\mathrm{L} \\
\mathrm{H} \\
\mathrm{H}\end{array}$ & $\begin{array}{l}1,2,3,4 \\
1,2,5 \\
1,2,3,4,6\end{array}$ & Planned move to a web-based product using. Net and MS-SQL server. \\
\hline 2 & $\begin{array}{l}\text { Market orientation (M0) } \\
\text {-information sensing } \\
\text {-information dissemination } \\
\text {-organisational response }\end{array}$ & $\begin{array}{l}\mathrm{M} \\
\mathrm{H} \\
\mathrm{M}-\mathrm{H}\end{array}$ & $\begin{array}{l}2,3,4,5 \\
2,3,4,5 \\
2,3,4,5\end{array}$ & $\begin{array}{l}\text { Medium to high levels of MO. Offshore model; follows quarterly } \\
\text { work schedules from its US parent. }\end{array}$ \\
\hline 3 & $\begin{array}{l}\text { Learning orientation (L0) } \\
\text {-commitment to learning } \\
\text {-open-mindedness } \\
\text {-shared organisational vision }\end{array}$ & $\begin{array}{l}L \\
L \\
M-L\end{array}$ & $\begin{array}{l}1,2,3,4,5 \\
1,2,3,4,5 \\
2,3,4,5\end{array}$ & $\begin{array}{l}\text { Limited evidence of critical thinking. People are recruited to } \\
\text { work in a narrow range. }\end{array}$ \\
\hline 4 & $\begin{array}{l}\text { Quality management systems (QMS) } \\
\text {-Commitment \& sharing information } \\
\text {-continuous improvement } \\
\text {-teamwork }\end{array}$ & $\begin{array}{l}\text { M } \\
M \\
M-L\end{array}$ & $\begin{array}{l}2,3,4,5 \\
1,2,3,4,5 \\
2,3,4\end{array}$ & ISO-certified processes, plus other processes developed by the US. \\
\hline 5 & Employee turnover & $\mathrm{H}$ & $1,2,3,4$ & $\begin{array}{l}\text { Higher than industry average. Testing roles, uncompetitive } \\
\text { wages and monotonous and shift work. }\end{array}$ \\
\hline 6 & $\begin{array}{l}\text { Enterprise size, complexity and } \\
\text { ownership }\end{array}$ & $\begin{array}{l}\mathrm{M}, \mathrm{M} \text {, } \\
\text { and MNC }\end{array}$ & $\begin{array}{l}1,3,4,6 \\
1,2,3,4 \\
2,3,4\end{array}$ & $\begin{array}{l}\text { Small single product organisation. Product knowledge and } \\
\text { US parent's mandate for focused training only. }\end{array}$ \\
\hline 7 & Temporal dimension & M-L & $2,3,4,5$ & $\begin{array}{l}\text { New product releases or customisation requests have minimal } \\
\text { impact on training volume and diversity. }\end{array}$ \\
\hline 8 & Geographical dimension & $\mathrm{N}$ & $2,3,4,5$ & Only US and Canadian clients. Technology cuts across regions. \\
\hline 9 & $\begin{array}{l}\text { Strategic HRM approach and workforce } \\
\text { composition } \\
\text {-rewards \& performance management systems } \\
\text {-career planning } \\
\text {-recruitment skills level \& operating roles; and } \\
\text {-use of non-standard employment }\end{array}$ & $\begin{array}{l}\mathrm{H} \\
\mathrm{L} \\
\mathrm{H} \\
\mathrm{N}\end{array}$ & $\begin{array}{l}1,2,3,4 \\
1,2,3,4,5 \\
1,3,4,5 \\
1,2,3,4,5\end{array}$ & $\begin{array}{l}\text { Uncompetitive salaries, strong performance management, } \\
\text { focussed recruitment, and limited career development opportunities } \\
\text { have a varying impact on training. } \\
\text { Permanent workforce, } 95 \% \text { in operational roles. }\end{array}$ \\
\hline
\end{tabular}

Legend: $\mathrm{H}=$ High; $\mathrm{M}=$ Medium; $\mathrm{L}=$ Low; $\mathrm{N}=\mathrm{Nil}$

\section{Discussion}

Based on the above analysis, different factors have varying levels of impact on the nature and extent of training. Organisation I's low LO and medium MO result in incremental 
and adaptive learning. Its strong product mindset and a large market potential affect its medium MO and low LO. However, recent changes in its product market require it to move to a web-based platform and accommodate customisation requests from its clients. This shift in clients' needs has been recently noted at the ODC, wherein its project managers are now working together with its US team to sense clients' customisation needs and to frame operational and training solutions. To help the ODC realise its full potential, such changes ought to be supported by further investment in soft skills and QMS, such as CMM Level-5, which it plans in the near future.

The more it moves from selling products to services, evident in the form of implementing customised solutions for its clients, the more its LO, MO and QMS will need to be developed. Thus, necessitating the development of new skills and learning to demonstrate generative or higher order learning. Building higher order learning and expertise would also enable ODC teams to engage in high-end application design and development, which is currently the focus of the US team. In this case, the training function was embedded in the operational teams, thus pointing towards a more operational role for training in such environments. Its HR infrastructure, from a training perspective, focuses more on developing skill inventories and monitoring the training budget.

\section{Conclusion}

A cost-reduction strategy provides a strong rationale for setting up the ODC. Overall, the case study shows how training decision making is influenced in a small software product ODC. It demonstrates that the work of a low-end nature and product environment requires low levels of LO and MO capabilities. Consequently, the organisation is mainly investing in process- and product-specific and limited soft skills training for meeting its immediate codevelopment needs. 


\section{CHAPTER 5.10}

\section{ORGANISATION $\mathbf{J}$}

\section{About Organisation J}

Set up in 2004, Organisation $\mathrm{J}$ is a small Bangalore-based captive offshore process centre (OPC) and an offshore development centre (ODC) for a large US-based SAP (an enterprise resource planning (ERP)) software solutions organisation. Organisation J's parent organisation, a SAP solutions provider, operates from multiple locations in the US and provides SAP-ERP maintenance, implementation, and support services and solutions. It specialises in providing SAP solutions to 11 industry sectors in the US, including manufacturing, banking and finance, insurance, chemicals, oil and gas, hi-technology, and federal and state governments. Within these industry sectors, it offers solutions in numerous specialisations using a range of SAP enterprise products, such as mySAP Business Suite, mySAP CRM (customer relationship management), mySAP SRM (supplier relationship management), mySAP SCM (supply chain management), mySAP PLM (product lifecycle management), mySAP ERP (which includes workforce planning, financial services, operations and corporate services), and SAP NetWeaver (which enables organisations to unify various technology components into a single platform).

The parent organisation's Indian subsidiary, Organisation J, employs about 36 people at its Bangalore facility, of which about 15 are in the OPC and the remaining 21 are employed in ODC. Its country manager, India Operations explained:

\footnotetext{
It's not just a back office. Back-office work is, I would say, just $40 \%$ of the work; $60 \%$ of the work is value-add, services like content creation, website creation, maintenance . . . all this is done from here, financial analytics, you know, those sort of things. OPC, that's processing. OPC is a captive, ODC is not a captive, ODC is part and parcel of the core business.
}

OPC focuses on recruitment and marketing processes for its US parent organisation, whereas ODC focuses on providing complete SAP solutions, from selling SAP products and licences to consulting and implementing solutions, through to post-implementation maintenance and support for the above noted industry sectors and specialisations. It helps clients in customising SAP applications and providing solutions to cater for their specific needs. 
The commonly understood purpose of an ERP application is to bring about an enterprisewide integration of business processes, data, and units into a unified system, with an expectation of bringing about efficiencies and possibly enhancing organisational decisionmaking capabilities. It reduces multiple and disparate software applications in an organisation into a single interface application and helps in standardisation. For one example, SAP ERP provides integration of two or more business functions, for example, payroll and accounting or inventory and sales.

Being a strong global ERP product, SAP has numerous services and solutions providers in India, and Organisation $\mathrm{J}$ is one of the many providers in the industry. In addition, SAP worldwide has had operations in India since 1996 and currently employs over 3500 people. Thus, in the last decade or so, there has been an accumulation of a critical mass of SAP-trained and -experienced professionals in the Indian labour market, making it attractive for SAP service providers worldwide to open ODCs like Organisation $\mathrm{J}$ in India.

\section{Organisation Structure and Processes}

Organisation $\mathrm{J}$ started off with 15 staff in 2004, and there are now 36. Rather than adopting an ethnocentric approach, it made a conscious effort to learn from its own mistakes, as some US practices were not applicable in the Indian cultural context and its wider labour markets. Most of its employee growth has been in the ODC business line. In the last 2 years of operation, it has streamlined its systems and processes and its order book position is firming up. Its parent organisation offers enterprise solutions to customers at various stages of their ERP applications' life cycle and transfers only certain projects to its Bangalore facility. The typical work structure of its ODC team is based on SAP specialisations. For example, entry level SAP developers are supervised by a lead developer (LD). The LD is supervised in turn by an assistant manager and a practice head $(\mathrm{PH})$, who heads the entire team. The $\mathrm{PH}$ has considerable technical and domain knowledge and experience. At any given point, the span of control does not exceed 1:4. Similarly, the OPC structure has four levels, process co-ordinator, analyst, manager, and PH. Specialisations are grouped on the technical and domain expertise of teams. 


\section{Business Processes, Strategy, and Work Organisation}

Organisation J's business development function is undertaken by its US parent, so there is minimal client interface between Organisation $J$ and its clients. Business is secured from clients who either have acquired and implemented a SAP-licensed product from a SAPauthorised business partner and are looking for enhancements and/or maintenance and support vendors or who would like to engage a solutions provider for a partial or an endto-end consulting, implementation, support and maintenance project. The nature of the projects undertaken includes fixed price, fixed scope, and time and materials projects. Broadly, its solutions can be grouped into consulting solutions, customisation solutions, and post-implementation maintenance and support. Consulting includes three key aspects: designing systems architecture, business process consulting (or business process reengineering), and technical configuration to allow for clients' business needs. Customisation is usually offered when the applications' existing modules or so-called "best-practice" modules are not suitable for clients' requirements. It requires customising the applications' functionality and features to suit the client's current and future needs, given that customisation projects are usually time-consuming, and the nature of the contract is usually time and materials with average costs ranging from US\$180-200 per hour. Here, the solution provider's domain expertise and industry knowledge, experience, and capabilities are of extreme importance. Organisation J's country manager, India stated:

\footnotetext{
We have [globally] 500-plus people, who are hard core SAP, with 8 years plus of experience. We have experience in all verticals. Today it's a question of who will focus on what verticals rather than any specific verticals. . . . Our people are our IP [Intellectual Property].
}

The nature and extent of the project complexity depend on whether a client is looking for a short-term project or a full-scale end-to-end solution. The project metrics and SLAs for delivery are negotiated accordingly. As its parent organisation has considerable SAP product experience, and domain and industry knowledge, it has the ability to negotiate fixed-price and fixed-scope projects, although most customisation projects are time and material projects. 
Regarding it business strategy, Organisation J's country manager stated:

You will say I want to attack these top ten companies in this industry in this area, typically look at revenues from this to this, try to find out what is their current IT radius. If they have this already there, ignore them. So strategy cannot be broad strokes. It has to be very aesthetic, boldly and aesthetically.

Client specifications and SLAs are negotiated by the US parent organisation. Organisation J provides analytic support through its OPC. The broad brief for Organisation $\mathrm{J}$ is execution of the projects undertaken (by ODC) and providing necessary process support and delivery (by OPC). The nature of services offered typifies a slightly differentiated services market (Boxall, 2003).

\section{Nature and Extent of Training Provision}

The training provision for both business lines (OPC and ODC) is different in both nature and extent. Figure 25 outlines the key emphases of training at Organisation J. Currently, the organisation does not have a dedicated training infrastructure as it is small, still evolving, and in the process of establishing its systems and processes. Thus, most learning and development is informal and incidental in nature. It has identified subject matter experts (SMEs) who act as mentors and coaches for its newly inducted employees. For instance, for its OPC, when a new employee starts, basic knowledge is provided in an orientation programme by SMEs. As noted above, OPC has a mix of value-added and non-value-added processes, and the focus initially is on developing process knowledge using its proprietary tools. Once the basic modules are covered employees are assigned to a mentor. Its country head- India, operations explains:

\footnotetext{
We have identified approximately two to three people at each level and department who are big brothers and big sisters. The minute any new employee comes in they are put onto one of those identified people. That buddy will see to it that everything about the process is clarified.
}

By means of its proprietary process automation and tracking tools, information about a new trainee's learning is recorded and used by others to find out the trainee's further training needs. For ODC, it is slightly different; after orientation, employees undergo detailed SAP technology training. The focus here is not just on technology, but also on the processes and delivery methodologies. 


\begin{tabular}{|lllll|}
\hline $\begin{array}{l}\text { New Hire } \\
\text { training }\end{array}$ & $\begin{array}{l}\text { Process, } \\
\text { application \& } \\
\text { technology training } \\
\text { (OPC\&ODC) }\end{array}$ & $\begin{array}{l}\text { Domain (ODC) } \\
\text { \& proprietary } \\
\text { tools training } \\
\text { (OPC\&ODC) }\end{array}$ & $\begin{array}{l}\text { Advanced } \\
\text { technology } \\
\text { \& soft skills } \\
\text { training (ODC) }\end{array}$ & $\begin{array}{c}\text { Coaching } \\
\text { \& } \\
\text { mentoring }\end{array}$ \\
\hline $\begin{array}{l}\text { 1 week } \\
\text { Informal } \\
\text { Process specific }\end{array}$ & \begin{tabular}{l} 
Formal \\
\multicolumn{7}{|l}{ Fo to 6-8 weeks }
\end{tabular} & $\begin{array}{l}\text { 2-3 weeks } \\
\text { Project dependent }\end{array}$ & $\begin{array}{l}\text { 1-2 weeks } \\
\text { Project } \\
\text { dependent }\end{array}$ & High \& ongoing \\
(Mostly informal for OPC; some training is highly structured for ODC business line.)
\end{tabular}

Figure 25. Temporal dimension of training: Changing emphases.

Next, employees are grouped based on their roles and assigned to technical SMEs for another week of training in their specialist areas. It employs specialist external training providers for technical training. All trainees are then tested in a simulated learning environment and work on dummy processes to check if learning is internalised and effective. Any gaps in their learning are identified informally and further training provided. An ODC training programme lasts for 6-8 weeks, compared to 3-4 weeks for OPC. The exact duration and nature of the training depends on the processes and specialist technology areas. In addition to the above, client-facing groups are given communication skills and soft skills training in both OPC and ODC groups. The extent to which the organisation provides technical and generic training for ODC and OPC are summarised by the country head below:

For ODC:

I would say, three-quarters is technology-orientated, more, maybe $80 \%$; only $20 \%$ is very generic, because I would count even processes is a part of your technology. Their processes are very different .... Soft skills, it is a very strategic thing. At a soft-skill level we don't make a distinction between one for technology team and one process team. When we do that we just put the whole caboodle in that, because we already know who are all the people who are going to be close to clients.

For OPC:

No, there it's more of 60/40 (technical/generic or soft skills). Two-thirds are processes because for them to know about where the company is positioning its strategy is very important because that has to translate into their work. It's very important. 


\section{Analysis of Factors Influencing Provision of Training}

\section{Workplace Change}

The change in technology from ABAP (Advanced Business Applications Programming) language to SAP NetWeaver has had a major influence on its training needs. Its recruitment strategy now focuses on hiring people with SAP NetWeaver knowledge, skills, and experience. This focus is critical given its small size and limited training infrastructure. It develops skills through the mentoring and coaching approach of its existing pool of trained and experienced personnel.

In late 2004, as the organisation was establishing processes through trial and error; it did not have a theory-in-use. Although the US parent set the tone and the overall strategic focus, Organisation J contextualised its systems and people management practices to suit the Indian cultural environment. Doing so particularly affected its recruitment and remuneration practices, which included abandoning the concept of non-standard employment and a high variable pay. This change is now reflected in its workforce composition of full-time permanent employees on a fixed salary package. Getting it right is critical for small start-ups like Organisation $\mathrm{J}$ because it has budgetary and operational constraints. Since its inception, it has learnt from its mistakes, thus exhibiting a culture of open-mindedness (albeit limited).

\section{Quality Management Systems (QMS)}

Although there is no formal quality infrastructure or accreditation, Organisation $\mathrm{J}$ has its unique quality methodology for SAP applications and uses its proprietary systems and tools to monitor its work flow, processes, and metrics for the quality of deliverables. One of the respondents explains:

\footnotetext{
See, the beauty about this system is that it is not only a process backbone, but it's also [a] quality monitoring tool. You don't capture it unless the process has been done, so we have made it the most fundamental requirement of the jobs. . . . In a person's job description, everything has to be through this process. If you don't, it's counted not done.
}

The processes require collection of project- and process-specific data. Because Organisation $\mathbf{J}$ is a small and evolving firm, it currently focuses more on fulfilling the 
clients' expressed SLAs, but is gradually beginning to focus on building process maturity capabilities. Accreditations such as CMM are planned for later in its growth cycle. One respondent stated:

\footnotetext{
We have our own processes. We do intend to go in for some quality certification, because in certain markets that we expand, as we go, Europe and all those places, they are asking, what sort of quality certifications do you have? ... In the next 18 months we will definitely be either an ISO- or CMM-certified; at least, especially for India it is a priority.
}

Its MO ability is strengthened by the use of its proprietary tools and systems, since clients' specifications are captured and disseminated in real-time using this system. Thus, its internal quality management processes are integrated at the various stages of information sensing, dissemination and framing a response. Its LO ability is also strengthened by the use of its quality systems and proprietary tools, wherein employees post queries, experiences and solutions about technology-related issues. Others contribute by critiquing the technology or building on the existing comments. Consequently, a small community of learning has started to emerge and people are beginning to use it as a resource for routine queries and enhancing their knowledge.

\section{Market Orientation}

Organisation J's MO ability is geographically and functionally distributed between the US and India. The project scope for OPC and ODC is driven largely by client requirements. Although its business strategy sets the tone for information sensing and the nature of projects to be undertaken, the bulk of this activity happens in the US and there is limited interaction between business development teams in the US and Indian operations at the time of signing new projects. However, its information dissemination is very strong because all client specifications that are sensed are disseminated real-time, through its proprietary systems and tools, in a way that enables all project team members to have a clear understanding of the project requirements. Tracking of project status, resources, key skill sets, and the number of people needed is done with the use of its automated proprietary tools. The country manager explained the process:

Everything is available as a cross-sectional slice, down the middle slice. Just slice and dice and get analytical. . . . Let's say IBM is the client, so our client module has IBM; it has the business intelligence and related information and updated contact lists, various places and people with whom we do our business. There will be the contact sheet, as soon as we get a 
requisition or a project for staffing, or requirements from one of these people. It gets linked to them, goes into opportunity module. It creates an opportunity for us, let's say we need 24 ABAP-skilled people. That goes to all our recruiting people. So once that goes to them, they take the people from their own system, which is the candidate module, and put a system through the opportunity module, maybe call it opportunity, and show the opportunity module it is submitted back to SAP or their client or, in this case, IBM.

The above system offers tight project management schedules and allocation of responsibility. Accordingly, project responses for business requirements and timelines are framed. The system also identifies the skills gap for a given project and considers external recruitment, in-house training, or a mix of both.

\section{Learning Orientation}

Owing to its still evolving nature of business processes, Organisation $\mathrm{J}$ is striving hard to fully articulate its theory-in-use through ongoing trial and error and learning processes. While client specifications are captured through its proprietary tools, its ability to bring about higher order learning will occur with a high LO ability and open-mindedness to deliver better service and ensure productivity gains. Organisation J does challenge some of its clients' and business development teams' assumptions, but only to a limited extent. A manager noted:

Every morning the sales and recruiting team gets together for one hour and they discuss all the high priority tasks that we do, you know, the projects, processes, etc. So a lot of the times these process-related issues come out right then and there .... We typically end up, you know, you, instead of giving a time frame of $25 \%$ of the project, I give you $5 \%$. Please don't over-commit. Why would a sales person give a guarantee to your customer for a 1week turnaround for a 24-person team? It's impossible.

The same manager described information sharing among different teams in India and the US and the clients:

See, we have this morning call, we have weekly digital call, where all the decoders in the region, reporting, consulting, and the sales people get together, and just get together to discuss the real issues. Again, there are some issues around so people get pulled in. So the director of, you know, the Board might be opposing, so the person who heads the recruiting function and some of the employees get pulled in. . . Well, there is lots of process questioning ... definitely, this will be because people have the right to say, it's a democratic process. 
Its commitment towards learning is gradually developing to establish a learning and development infrastructure. Currently, the focus is on providing essential training driven by clients' project requirements. Its ability to challenge clients is also affected by the nature of work received. In some cases, tight project requirements allow no latitude. Client specifications and nature of work influences the extent to which between LO and MO capabilities can be fully developed. One respondent noted:

\footnotetext{
See, technology side is only determined by the client's requirement, what part of the system they are exposed to, what are their requirements; everything is very client-directive. I am talking in terms [of] performing their job, specific tasks. OPC is gradually different that way-tactical environment. In ODC, we give them all that necessary access. Obviously it does not mean that that they can go and change everything and anything in the system. So whatever we need to do to the jobs in terms of logic we do that.
}

Through the use of proprietary tools for and through informal mentoring and coaching, employee development needs are addressed and captured. Overall, Organisation J appears to have medium to low levels of LO.

\section{Employee Turnover}

Owing to its small size, employee turnover does not seem to be of great concern to Organisation J. It is around 15-20\% per annum and does not appear to have any significant impact on training, except for its orientation and the subsequent product training that follows. As noted earlier, its small cohort of new employees learn in an informal and incidental manner. Such turnover levels are considered to be healthy by the organisation and are seen as a means of organisational renewal. Any training needed as a consequence of employee turnover at this level is considered as a "necessary evil". It was noted by one of the respondents that the organisation would benefit from the new learning and knowledge that new employees bring, a view consistent with Banerjee's (2004) notion of seeking profit from information-in-expectation from new hires.

\section{Enterprise Size, Complexity, and Ownership}

Because it is a small start-up, the focus of the organisation is on ramping up an operational facility with a resource base of SAP professionals. With further expansion 
planned, the organisation has committed to expanding its HR function for 2006. The country manager-India said:

[The] HR manager's mandate is to come up with a development planning document for the company and specific event-related definitions, and we'll have our culture-mapping training, technology training programme, safety training programme, and something on leadership development. A document is being developed. Yes, because now we have people, we have the critical mass to actually plan long-term. Now, we can be much more proactive because we've got a base and we know our people, and we know our daily routines, and we have them in substantial numbers.

Currently, the nature of projects is not complex, but the organisation anticipates that in the near future more complex and demanding projects will be executed, which will have an impact on its recruiting strategies, and training and skill requirements. Ownership has had a moderate impact on the formalisation of training, as is evident in the use of its proprietary tools.

\section{Temporal Dimension}

Although training needs were initially high (in 2004) for its OPC service line, they have now stabilised. Low employee turnover has further helped in containing employee costs and training volumes. Training volume is not affected by the temporal dimension of business processes. The country manager explained:

\footnotetext{
No, because OPC is a very simple thing, because stakeholders are within [the] company itself. The initial bump is there when you have to invest in a lot of time, and I must say the effectiveness is very low to start with. Only thing that we are learning is the new organisation. But now in 2005 we are pretty straight and systems are in place.
}

For ODC, since most projects have their unique business requirements in terms of skills and number of people, Organisation J's use of a focused recruitment strategy helps in minimising its investment in technical skills training.

\section{GEOGRAPHICAL DIMENSION}

All its clients are based in the US, and a single product technology is used in projects. Movement within the US markets does not appear to have a significant impact on the nature and extent of training. However, one respondent noted that demand would increase 
if they moved to European markets as there would be a need to understand the business knowledge and logic of European markets. Further, because the project teams have significant work experience in the US markets, learning has consolidated. The country manager for India noted:

I anticipate that we then need to identify, train, and maintain the set of resources focused more on Europe processes and projects. I think it's a little difficult to have a pool of resources which knows everything about everything. Maybe we might be better off having a core team which specialises in Europe.

\section{Strategic HRM Approach, Skills Level, and Workforce Composition}

Organisation $\mathrm{J}$ has recently appointed an HR manager. Prior to this, HR was undertaken by PHs with input from the country head. Processes for recruitments, performance management systems and formal training programmes are now being developed with its existing proprietary tools and systems. Its country head noted:

\footnotetext{
It's very easy for us to figure out what is wrong, what needs to be done. So, on that basis . . so we don't wait for like two months to connect 10 people to send to a programme. We find one guy lacking on one area; we find out whether it's a process problem or whether it's a person problem. If it's a process problem, we fix the process; if not, talk to the person. Go and find out whether it's a training issue, or it is a discipline issue. This is the way you should tell them: we didn't expect you to do this thing. You may be good, you might be doing well, you got to have [the] discipline to enter this into the system. So we know you are doing it. We don't know you are doing it because you are not making it obvious.
}

Given the cultural and contextual factors of the Indian labour market, all employees are on full-time permanent employment contracts. Thus, it was not possible to establish any relationship between casualisation or permanency and training. Recruiting skilled and experienced employees has a negative impact on the volume of training. However, there is still some need for organisation-, client-, and domain-specific training to be provided. A closer analysis of the types of recruitment the organisation is focusing on reveals that it is on building domain-specific expertise, using SAP applications. Typical examples of recruitment for Bangalore include SAP PP (Production Planning), MM (Materials Management), Sales, Finance, and HR consultants. For all these roles an experience range of 3-6 years is sought. For its technology roles, the minimum experience for SAP consultants is 5 years or more, with tight specifications for specific SAP modules. 


\section{Specific Impact of Factors on Nature and Extent of Training}

The following section provides an analysis of how various factors affect the nature (externalisation, diversity, transferability, and formalisation) and extent (volume) of training. As Miles and Huberman (1994) suggest, a conceptually clustered matrix is the first step in developing explanatory networks, because it provides a snapshot of the intensity and direction of the different factors under consideration (see Table 15) and includes notes from the researcher. It also shows the extent to which various respondents observed a phenomenon.

\section{Volume of Training}

On average, employees in OPC receive 3-4 weeks of training, as against 6-8 weeks in ODC. Its training volumes (see Figure 25) in ODC are driven directly by SAP applications knowledge and the project specifications it receives from its clients. For ODC, its focus is on either finding an exact match of the skill sets required or providing training of up to 8 weeks. Although the training volume has increased with growth in employee numbers, the extent to which it can be quantified is blurred by the informal learning that is provided. Further, the training volume varies according to the level of skills the organisation is able to recruit from the external labour market.

\section{Nature of training}

In terms of training diversity, the bulk is technical and focuses on product knowledge of SAP modules. As the organisation is currently undertaking less complex projects, its investment in advanced technical, domain-, and industry-specific training is limited. Knowledge of its proprietary system and quality monitoring further increase training volume and diversity, and they are likely to increase further when the organisation seeks for an external quality accreditation standard. Its current quality monitoring system is also a performance management tool, whereby any gaps in performance are identified and the necessary training provided. In terms of technical versus generic, the focus is more towards developing technical and project-specific training. There is limited support for soft-skills development or for further education. Investment in technical training and generic skills is likely to increase when the organisation procures projects with high 
complexity and specifications and when its need to interact directly with clients increases.

Table 15.

Conceptually Clustered Matrix of Factors

\begin{tabular}{|c|c|c|c|c|}
\hline S.No. & Main factors and attributes & Intensity & Respondents & Researcher's notes \\
\hline 1 & $\begin{array}{l}\text { Workplace change } \\
\text {-structural } \\
\text {-ownership } \\
\text {-technological }\end{array}$ & $\begin{array}{l}\mathrm{H} \\
\mathrm{L} \\
\mathrm{H}\end{array}$ & $\begin{array}{l}1,2 \\
1 \\
1,2\end{array}$ & $\begin{array}{l}\text { Numerous iterations in its systems \& structure. NetWeaver and } \\
\text { emerging technologies require considerable up-skilling. }\end{array}$ \\
\hline 2 & $\begin{array}{l}\text { Market orientation (M0) } \\
\text {-information sensing } \\
\text {-information dissemination } \\
\text {-organisational response }\end{array}$ & $\begin{array}{l}\mathrm{M} \\
\mathrm{M}-\mathrm{H} \\
\mathrm{M}-\mathrm{H}\end{array}$ & $\begin{array}{l}1,2 \\
1,2 \\
1\end{array}$ & On average, it has medium-to-high levels of M0. \\
\hline 3 & $\begin{array}{l}\text { Learning orientation (L0) } \\
\text {-commitment to learning } \\
\text {-open-mindedness } \\
\text {-shared organisational vision }\end{array}$ & $\begin{array}{l}\text { M-L } \\
M \\
M\end{array}$ & $\begin{array}{l}1,2 \\
1,2 \\
1,2\end{array}$ & $\begin{array}{l}\text { Limited evidence of critical thinking is present. It is also affected } \\
\text { by tight client requirements. }\end{array}$ \\
\hline 4 & $\begin{array}{l}\text { Quality management systems (QMS) } \\
\text { - Commitment \& sharing information } \\
\text {-continuous improvement } \\
\text {-teamwork }\end{array}$ & $\begin{array}{l}\mathrm{M} \\
\mathrm{M} \\
\mathrm{H}\end{array}$ & $\begin{array}{l}1,2 \\
1,2 \\
1\end{array}$ & $\begin{array}{l}\text { Well-developed process monitoring and automation tools serve } \\
\text { as its quality backbone. }\end{array}$ \\
\hline 5 & Employee turnover & $\mathrm{L}$ & 1,2 & $\begin{array}{l}\text { Small firm size. Low employee turnover; considered good for } \\
\text { organisational renewal. }\end{array}$ \\
\hline 6 & $\begin{array}{l}\text { Enterprise size, complexity and } \\
\text { ownership }\end{array}$ & $\begin{array}{l}\mathrm{L}, \mathrm{M}-\mathrm{H} \\
\text { and } \\
\mathrm{MNC}\end{array}$ & 1,2 & $\begin{array}{l}\text { Small-sized organisation where informal learning is strong; } \\
\text { moderate influence of ownership on training, medium } \\
\text { complexity projects, } 11 \text { verticals and multiple domains increase } \\
\text { skills requirements. Most are met by specific recruitments. }\end{array}$ \\
\hline 7 & Temporal dimension & M-L & 1 & $\begin{array}{l}\text { SLAs changes, technology and product upgrades impact on } \\
\text { training. Initial bump in training was noted in 2004-2005. }\end{array}$ \\
\hline 8 & Geographical dimension & $\mathrm{L}$ & 1 & Currently all projects are from the US markets. \\
\hline 9 & $\begin{array}{l}\text { Strategic HRM approach and workforce } \\
\text { composition } \\
\text {-rewards and performance management systems } \\
\text {-career planning } \\
\text {-recruitment skills level \& operating roles; and } \\
\text {-use of non-standard employment }\end{array}$ & $\begin{array}{l}\text { M-L } \\
\text { M-L } \\
\text { H } \\
\text { L }\end{array}$ & $\begin{array}{l}1,2 \\
1,2 \\
1,2 \\
1\end{array}$ & $\begin{array}{l}\text { Small HR infrastructure. PMS is still evolving. Uses its proprietary } \\
\text { tool for work flow monitoring and training needs identification. } \\
\text { Highly focused recruitment and selection for ODC. } \\
\text { No use of temporary and part-time. }\end{array}$ \\
\hline
\end{tabular}

Legend: $H=$ High; $M=$ Medium; $L=L o w ; N=N i l$

On analysing the skills and experience profile of its new hires, it appears that the focus is on acquiring diverse domain-specific skills. Consequently, the diversity of its current informal learning is likely to increase for project team members. 
Technological change is evident in an increased demand for NetWeaver projects, which in turn requires a new set of technical skills to be developed. SAP NetWeaver skills and experience are both hard to find and attract, even with higher wages. Its training diversity varies according to the specific skills levels and experience it can hire. QMS at Organisation $\mathrm{J}$ focus on inculcating process and project management discipline in its employees. Some generic project management and quality metrics have been developed that need to be covered via employee orientation and technical and project-specific training. As noted by one of its respondents, QMS will have a positive impact on the nature and extent of training when the organisation prepares itself for either a CMM-level or an ISO 9000 certification.

As noted above, the bulk of the OPC training is provided internally and is mostly informal and technical in nature. The organisation's small size and relatively less complex nature of projects are reasons for not investing in a training infrastructure. Reliance on external training providers is limited to specialist technology areas such as NetWeaver technologies and to developing soft skills for client-facing groups. The organisation anticipates that, as its QMS develop, its initial reliance on external training vendors for quality management training will increase.

Its ODC service uses its proprietary systems for managing project quality and performance and formally monitors, assesses, and delivers technology training. However, for $\mathrm{OPC}$, it has an extremely low level of training formalisation.

\section{Discussion}

The factors influencing training provision at Organisation $\mathrm{J}$ include project [client] specifications, quality management systems, and technological change. The nature of, and extent to which, such training is provided are affected by its performance management systems, skill levels of the employees it hires, project complexity, and the temporal dimension of its process and projects. LO and employee turnover have a limited impact on training. Its $\mathrm{MO}$ ability and proprietary quality and project management systems enable sensing and dissemination of client/project specifications. This information enables identification of gaps in skill sets. Technological change (demand for NetWeaver technology projects) has had an impact on the demand for new skills. It is expected that as 
SAP (globally) rolls out new products or product enhancements, the organisation will need to train its staff to ensure that they are familiar with changes to product functionalities.

Its medium to low LO and lack of any formal quality management accreditations affect its ability to undertake higher order learning. Consequently, it demonstrates adaptive learning. While a high MO is desirable, on its own it restricts the development of the organisation's internal capabilities, unless accompanied by a high LO and strong QMS. A high LO is possible if a culture that supports new ideas and challenges the status quo is promoted. Further, because the focus of its current QMS is on bringing about process efficiencies and project management discipline within a given SLA framework, its ability to enhance the organisation's overall project management capabilities and process maturity is limited.

The effect of firm size on training provision is masked by the low complexity of the projects it currently services and the high level of informal and incidental learning that happens at the workplace. The above analysis also suggests there is no impact from changes in clients' geographical locations because the organisation currently secures all of its business from the US. Organisation J's HR infrastructure, which is recently been set up, shows strong evidence of a focused approach to recruitment, supporting the "buy", rather than "make" strategy.

\section{Conclusion}

Organisation J's current recruitment focus suggests the need to develop domain-specific skills. Given the increasing number of SAP solutions providers and SAP India's expansion plans, the organisation will need to build, among other capabilities, a stronger internal learning and development infrastructure and move away from its current model of recruiting highly skilled and experienced SAP professionals at high market costs. If it develops an internal learning and development infrastructure, it can develop and train entry-level engineering graduates (or those at a lower level for SAP applications and technologies) to contain its HR costs.

Although a few specialist SAP-credentialed training providers fill some gaps, tertiary education programmes are still lacking in industry and domain knowledge of relevant 
programming technologies. Inclusion of some of these areas in their curriculum will benefit smaller players, such as Organisation $\mathrm{J}$, and enable them to utilise their limited resources more productively. 


\section{CHAPTER 6}

\section{CROSS CASE ANALYSIS}

\section{Introduction}

The purpose of this chapter is to present a cross case analysis by applying the analytical techniques outlined in chapter 4 . This chapter presents the patterns and themes of factors influencing training across the 10 cases and analyses the similarities and differences between them. Such an approach enhances the accuracy and validity of the analysis and allows for exploration of new patterns (Yin, 2003; Eisenhardt, 1989).

This chapter begins with an analysis of the nature and extent of training practised in the case organisations. Use of matrices, visual displays, and figures (see Figures 26 to 33 and Tables 16 and 17) allows for better analysis and understanding of the relationships between the nature and extent of training and different factors (Miles \& Huberman, 1994). Such an approach forces the researcher to look for replications (literal and theoretical) as well as consider rival views and provide explanations for rival patterns. Next, pattern matching of key factors across the case sites and their interaction with organisational capabilities in shaping the nature and extent of training are presented. Such an approach forms the basis for developing a theoretical framework (presented in Chapter 7) for understanding the phenomenon of enterprise training. This chapter concludes by drawing inferences from this analysis on the possible links between training provision and firm performance.

\section{Nature and Extent of Training}

\section{Extent of Training}

A major finding of this study is the diversity of measures employed by organisations in describing their volume of training. Most organisations studied did not employ direct monetary measures of training volume (such as training as a percentage of payroll) and other measures noted in the extant literature (such as extent of shop floor training or proportion of employees covered) (Betcherman et al., 1997; Dostie \& Montmarquette, 2007; Smith \& Hayton, 1999; Smith et al., 2002). The problem in measuring employer 
expenditure on training has recently been acknowledged in research from Australia (Smith et al., 2008).

The application of measures from extant literature to India's IT sector is problematic owing to the dynamic nature of the coordination that exists between service providers and their clients (Banerjee, 2004). The total training cost is hard to determine as some costs are included in the clients' project costs and such training is provided at a project level. Other training costs are funded by clients from their own resources, which may or may not be directly costed. In addition to a range of in-house training provided by some MNCs' offshore development centres (ODCs) (Organisations A, E, G, I, and J), additional training is offered by expatriates to staff in India. The cost of such training cannot be easily determined as it is usually in the form of internal transfers, which may or may not be exclusively earmarked under a "training" cost code. Finally, costing the "learning by doing" and informal and incidental learning (Watkins \& Marsick, 1992) that business development and project team members undergo during the project/process solutioning exercise is hard to compute.

Broadly, then, approaches to measuring the extent of training can be expressed in terms of time or money. Owing to the problems noted above, this thesis does not employ absolute monetary measures. Instead, it focuses on time, expressed in hours, days, or weeks per employee. Although such an approach does not capture the entire range of training activity, it covers a significant proportion of it.

The most commonly used measure of training volume in the Indian IT sector is number of days or weeks per employee. However, even the application of this measure was not straightforward; different groups of employees received different people days of training. For example, recent college graduates at large organisations (A, E, F, and G) received longer induction and training than lateral hires. Further, owing to the diverse nature of processes or projects that an organisation services, it is difficult to have a standardised training timeline for the entire organisation. Thus, a range is presented.

Training comprised mainly three types: the induction, project/process-specific training, and leadership and management development programmes (MDPs). It is important to note here that the term induction training has been used variously by organisations to describe the conventional human resource management induction, ranging from minimal 
employee orientation right through to an all-inclusive and exhaustive induction programme. The latter was evident in large organisations (A, E, F, and G). In addition to the above, few organisations ( $\mathrm{A}, \mathrm{B}, \mathrm{E}, \mathrm{F}, \mathrm{G}$, and $\mathrm{H}$ ) offered a minimum mandated number of training days or hours per annum for ongoing staff development. The focus of such training was on developing staff in certain role-based competencies, meeting their career aspirations, and managing employee turnover. A summary of the nature and extent of training provided can be found in Table 16.

\section{Nature of Training}

In this thesis, following extant literature (for example, Dawe, 2003; Dostie \& Montmarquette, 2007; Smith 1997; Smith \& Hayton, 1999; Ridoutt et al., 2002) the nature of training is assessed by four criteria. For reasons outlined earlier in chapter 5.0, learning support was dropped from the final analysis. The four criteria consider:

- whether there is reliance on internal or external training

- the extent to which the organisation has formalisation of its training activities

- the type of training - whether it is behavioural or technical

- the degree of transferability of the training.

Despite the diverse organisational settings, replications of the training arrangements were found. Brief descriptions of each criterion and their application to cases are presented as follows:

\section{Internal or External Training}

Internal training. Training that is provided internally, primarily using the organisation's existing resources and facilities, and the extent to which the organisation has a dedicated training infrastructure - evident in the form of a training facility and use of internal trainers. Overall, most case organisations provided some training internally. Apart from the strategic reasons outlined below for developing an internal training infrastructure, such an approach is also suitable for the typical adult learner in the Indian cultural environment, who prefers classroom-based instruction to self-paced and self-directed learning. Organisations A and F relied heavily on internal training and were looking at training as a strategic investment choice to minimise the overall costs of service delivery. Both these organisations had made significant training infrastructure investments in the 
early stages of their evolution and gradually added additional capacities and capabilities in a phased manner. The cost and capability barriers of these investments, their historical path dependence and social complexity, make it difficult to be imitated by a new player in the competitive environment of these firms (Barney, 1991; Leonard, 1998).

Organisations E and G, being ODCs of large ITSS MNCs, have a different focus. These firms decided to limit their fixed costs in learning and development and retain their flexibility to relocate operations, should there be a need to do so, owing to changes in the global geopolitical climate for offshoring. Following the prescriptions of a resource-based view of the firm, Organisations $E$ and $G$ run the risk of exposing their firm-specific knowledge through knowledge spillover, which can occur by employing external training vendors. Its path dependence and social complexity (Barney, 1991; Leonard, 1998) limits the extent of such exposures.

Medium to small enterprises (B, C, D, H, I, and J) had relatively limited resources for developing their own internal training infrastructures. Organisation B had an internal training infrastructure. Because they are relatively small in size and for reasons that follow, Organisations $\mathrm{C}, \mathrm{D}, \mathrm{I}$, and $\mathrm{J}$ relied highly on internal training provision. Organisation C's main area of operation was in a relatively new industry sector-real estate. With limited domain expertise available externally, it had to build its capabilities internally. Owing to a product environment and the need to protect its proprietary knowledge, Organisation I preferred internal provision of training. The outsourced vendor network model adopted by Organisation D needed certain specialist consulting and quality management skills that could be developed only internally. Reasons for Organisations H's reliance on external training providers are discussed below.

External training. In most cases, the reasons for reliance on external training providers were twofold: limited resources for developing a training and development infrastructure $(\mathrm{H}$ and $\mathrm{J})$ and the global geopolitical climate $(\mathrm{E}$ and $\mathrm{G})$. Case organisations that relied on external training providers $(\mathrm{E}, \mathrm{G})$ also had some form of internal training provision. For reasons noted above, Organisations $\mathrm{E}$ and $\mathrm{G}$, despite having their own corporate universities, relied significantly on external training providers for delivery of their content. Organisations $\mathrm{H}$ relied on external training provision because of its size and the evolving nature of its organisational capabilities. Similarly, Organisation J relied on a mix of informal and incidental learning (internal) and external provision. The need for 
specialist training and skills development is in areas where the organisation does not possess the internal capability. Such training focuses on new and emerging technologies, development of specialist consulting skills general leadership development, and people management programmes.

\section{Formalisation of Training}

Formalisation of training refers to an organisation's practices of formal training need identification, design, development, and assessment of all training provided. The extent to which an organisation demonstrated a formal approach to training was partly explained by the resources at its disposal, the influence of external regulations, and the degree to which its QMS were developed. The presence of a metrics culture, evident through an organisation's strong QMS, facilitated a formal and structured approach to the organisation's training need identification, design, development, delivery, and evaluation.

Organisations A, E, F, G, and I had high levels of training formalisation. Except for a small organisation (I), this finding concurs with Dawe's (2003) meta-analytic study of training in large Australian firms. Organisation I, being in a product environment and catering to the highly regulated health services sector, needed to formally comply with various medical laws and guidelines in the US (see for example, HIPPA and AAMT standards in chapter 5.4) to ensure all its employees understood compliance issues and were proficient in the various product modules of the software application they were working on. Organisations A, E, F, and G had highly developed QMS, requiring a formal and structured approach to learning and development. Further, as organisations E and G were relying on external training providers, they had to formally develop metrics to assess their training vendors' training performance. Well-developed QMS enabled the development of such metrics and included variable performance payments, with penalties for poor training delivery. The organisations' performance management systems formally captured key performance metrics based on client specifications, and any resultant training gaps were reported to the line and quality account managers so that training would be provided and the gaps closed.

The importance of an organisation's stages of evolution and its HR practices (Baird \& Meshoulam, 1988) are also relevant in explaining formalisation of training in the remaining five organisations. Organisations B and H's training infrastructure was being developed for formally assessing training effectiveness and developing certification for 
Table 16

Nature and Extent of Training

\begin{tabular}{|c|c|c|c|c|c|c|c|c|c|c|c|}
\hline \multicolumn{2}{|c|}{$\begin{array}{l}\text { Training characteristics } \\
\text { Extent (Volume) }\end{array}$} & A & B & $\mathrm{C}$ & $\mathrm{D}$ & $\mathrm{E}$ & $\mathrm{F}$ & $\mathrm{G}$ & $\mathrm{H}$ & $\mathrm{I}$ & $\bar{J}$ \\
\hline 1 & Induction (weeks) & $1-2$ & $1-2$ & 1 & 1 & 2 & 15 & $2-4$ & $2-6$ & 2 & 1 \\
\hline 2 & $\begin{array}{l}\text { Process/project specific } \\
\text { (weeks) }\end{array}$ & $3-12$ & $1-10$ & $1-3$ & $1-2$ & $6-8$ & $2-3$ & $4-6$ & $2-3$ & 1 & $3-8$ \\
\hline 3 & MDP (weeks) & $2-3$ & 1 & 0 & 0 & 2 & 2 & 2 & 0 & 0 & \\
\hline 4 & Total (weeks) & $6-17$ & $3-12$ & $2-3$ & 2 & 10-12 & 19-20 & 8-12 & 4-9 & 3 & 4-9 \\
\hline 5 & Classification & $\mathbf{H}$ & $\mathbf{M}$ & $\mathbf{L}$ & $\mathbf{L}$ & $\mathbf{H}$ & $\mathbf{H}$ & $\mathbf{H}$ & M-H & $\mathbf{L}$ & M-H \\
\hline 6 & $\begin{array}{l}\text { Mandated training (per } \\
\text { annum for all-post } \\
\text { induction) }\end{array}$ & $\begin{array}{l}80-100 \\
\text { hours }\end{array}$ & $\begin{array}{l}80 \\
\text { hours }\end{array}$ & & & $\begin{array}{l}80 \\
\text { hours }\end{array}$ & $\begin{array}{l}80 \\
\text { Hours }\end{array}$ & $\begin{array}{l}80 \\
\text { hours }\end{array}$ & $\begin{array}{l}40 \\
\text { hours }\end{array}$ & & \\
\hline \multicolumn{2}{|c|}{$\begin{array}{l}\text { Training characteristics } \\
\text { Nature (Diversity) }\end{array}$} & $\mathrm{A}$ & B & $\mathrm{C}$ & $\bar{D}$ & $\mathrm{E}$ & $\mathrm{F}$ & $\bar{G}$ & $\mathrm{H}$ & I & $\mathrm{J}$ \\
\hline 1 & Internal & $\mathrm{H}$ & $\mathrm{M}-\mathrm{H}$ & $\mathrm{M}$ & $\mathrm{M}$ & H-MIX & $\mathrm{H}$ & $\begin{array}{l}\text { H-MIX } \\
(50 \%) \\
\text { EACH }\end{array}$ & $\mathrm{L}$ & $\mathrm{M}-\mathrm{H}$ & $\mathrm{M}$ \\
\hline 2 & External & $\mathrm{L}$ & $\mathrm{L}$ & L-N & $\mathrm{L}$ & M-H & $\mathrm{L}$ & M-H & $\mathrm{H}$ & $\mathrm{L}$ & M-L \\
\hline 3 & Formalisation & $\mathrm{H}$ & $\mathrm{M}-\mathrm{H}$ & M-L & M-L & $\mathrm{H}$ & $\mathrm{H}$ & $\mathrm{H}$ & $\mathrm{M}-\mathrm{H}$ & $\mathrm{H}$ & $\mathrm{L}$ \\
\hline 4 & \multicolumn{11}{|l|}{ Training type } \\
\hline & \multirow{2}{*}{$\begin{array}{l}\text { - Technical (\%) } \\
\text { - Behavioural (\%) }\end{array}$} & 70 & 80 & 90 & 95 & $85-90$ & $85-90$ & 80 & 90 & 90 & $90-95$ \\
\hline & & 30 & 20 & 10 & 5 & $10-15$ & $10-15$ & 20 & 10 & 10 & $5-10$ \\
\hline
\end{tabular}


trainers. Train the Trainer certification programmes were conducted immediately following the induction and project-specific training to test employees' process/project understanding. Organisations $\mathrm{C}, \mathrm{D}$ and $\mathrm{J}$ were still in the process of developing their training and HR capabilities. Progress was affected by resource constraints.

\section{Training Type}

A commonly noted dichotomy in the training literature focuses on the extent to which an organisation invests in technical or behavioural training. Technical training directly enhances an employee's ability to perform core tasks of their job. For example, providing training on a technology or programming language would be considered technical for an IT professional's role, and knowledge of US accounting practices would be considered technical in an F\&A role in an ITeS/BPO services firm. Improving personal capacity for interpersonal communication, writing effective emails, problem solving, team work, leadership and people management skills are examples of behavioural training.

While the above classification is helpful, it is not always useful. Respondents often noted that some behavioural training can be technical owing to the focus of a role. For example, customer service and sales skills can be both technical and behavioural, depending on the role they are associated with. Thus, for the purpose of this study, training that directly supports an employee's ability to perform their immediate job tasks and activities is considered technical; whereas training that enhances an employee's ability to work effectively in the organisation's unique cultural environment and with its growth plans and values is considered behavioural.

Overall, the focus of most training was technical in nature and aimed at strengthening employees' immediate job performance. Examples of technical training varied with each organisation and the industry sector or technology area each was catering to. In ITeS/BPO firms, for example, Organisation D had a narrow focus on medical transcription standards and knowledge of tax and accounting standards in the US. Organisation A, which had its presence in numerous industry sectors and specialisations, provided training in voice services, non-voice back office accounting, HR, instructional design, IT support, quality management, consulting, and market analytics skills. Similarly, Organisation B had a mix of low-end customer service and sales skills training, and high-end in-depth financial analysis training for developing the sustainability ratings of firms in various sectors. 
Similar diversity in technical training was noted in ITSS (projects and product environment). For example, firms that were operating only in a product environment (Organisations $G$ and $I$ ) required a specialised and narrow set of skills suited to their products (for example, semiconductor and embedded software design skills in Organisation $\mathrm{G}$ ). Large firms (E and F), which were operating in a product and project environment, focused on developing competencies in a broad range of technology areas suitable for a broad range of industry sectors. Smaller or medium-sized firms (H and J) focused on developing technical competencies in a narrower domain.

Contrary to extant research (Smith et al., 2003; Smith \& Hayton, 1999), in the case organisations studied, quality management training was found to be an integral part of technical, rather than behavioural, training. By employing quality metrics or standards of performance, organisations can identify deviations in performance and focus on developing skills for improving performance in a narrow range of tasks and activities.

Most respondents viewed behavioural training as critical in supporting an organisation's growth plans and in enabling employees to interact with the organisation's clients. Organisations that were growing at a fast pace (A, B, E, F, and G) provided higher levels of behavioural training, as they needed to invest in developing leadership and managerial talent, business development, negotiation, developing a global mindset, cross-cultural training, and other soft skills for their growth and change management plans.

\section{Transferability of Training}

This aspect of training focuses on one of the predictions of human capital theory: that organisations will be reluctant to invest in general or transferable training compared with firm-specific training. The distinction between general and firm-specific training is not an issue for most employers, as the bulk of the training provided is generic and transferable in nature anyway and is provided because of a skills shortage. The firm-specific training provided was described as either too little (for example, a short orientation programme about the organisation and its evolution) or something that was difficult to measure or quantify. Firm-specific learning is the knowledge about an organisation's daily routines and processes embedded in its work culture. Such training is often provided on the job and informally. Firm-specific training is not likely to be fully transferable as the strategic milieu of each organisation is different and the content of such training is hard to codify. Further, as resource theorists argue, the nature of such knowledge is a result of an 
organisation's path dependence and social complexity (Barney, 1991; Leonard, 1998; Wernerfelt, 1984; Wright et al., 1994). Organisations A, E, F and G's strong work cultures and business processes are so customised to their specific strategic milieus that they create an economic barrier for replication by their competitors and thus can be viewed as a source of sustained competitive advantage.

An example of firm-specific training is Organisation A's ability to infuse and apply its Lean Six Sigma capabilities to almost every aspect of its service delivery and business routines. Although Six Sigma has a wider application within and outside the IT sector, it is how Organisation A deploys it and recombines it with other organisational capabilities that are unique and hard to fully replicate. Similarly, Organisation G's strong work culture, technology leadership (ability to miniaturise design and enhance the processing power of semiconductors), and high level of customisation of its business and technology processes make replication unviable.

Banerjee's (2004) job-switching thesis and the neo-human capital assumptions of Bartel and Lichtenberg (1987) together explain how training plays a critical role in the managing of costs of large organisations, such as Organisations A, E, F and, to a lesser extent, G. These organisations do not lose much from employee turnover at lower to middle levels, as employee turnover helps them to keep their labour costs down by enabling them to recruit more recent college graduates and manage the costs associated with wage increases in their internal labour markets. Further, as the comparative advantage of skilled employees declines because of knowledge accumulation, and as the capability and capacity of these organisations to train increase, they can recruit new hires from second and third tier colleges more cheaply and easily.

Neither the human capital theory (Becker, 1962) nor Acemoglou and Pischke's (1998a, b) neo-human capital theory assumptions fully explain the organisation's decision to invest in general or firm-specific training. The issue is at the heart of organisations' strategic milieus (both external and internal), the dynamic coordination that exists between a service provider and its clients, and the unique configurations of various organisational capabilities and practices that eventually shape the nature and extent of training. 


\section{Pattern Matching of Factors Influencing the Nature and Extent of Training}

The following section provides an analysis of various factors that influence the nature and extent of training. Table 17 provides a cross-case comparison of the intensity of various factors and their sub-elements. For ease of comparison and analysis, data about these factors have been reproduced from the tables in within-case analysis. The impact of competitive strategy in shaping a firm's strategic milieu is discussed first, next, the way competitive strategy informs the firm's HR strategy and in particular its skills strategy. Next, an analysis of key organisational values, behaviours and capabilities is presented to show how these individually and collectively shape the provision of training. Finally, the direct impact of certain organisational and external factors is presented.

\section{Competitive Strategy}

As noted in chapters 2 and 3, there are two broad divisions in the Indian IT sector: the product and services environments (Banerjee, 2004). Organisations adopt different business models (third-party outsourcing service providers, offshore development or process centres or a combination of both) for operating in each product or services market. Services constitute the bulk of business activity in both the ITeS/BPO and ITSS sub-sectors. Further, given the uneven profile of the IT sector (Heeks, 1998) and the high levels of performance achieved by certain large ITSS (product and services) and ITeS/BPO firms, some firm-level differences in performance cannot be ruled out (Ethiraj et al., 2005). These differences in performance can broadly be attributed to a range of strategic choices exercised by firms and to their development of certain organisational capabilities for achieving sustained competitive advantage or superior performance.

An organisation's strategy is best discerned by its behaviours or actions. These behaviours are driven by what a firm values most in terms of its competitive position in the market. Such a view is common in strategic management literature and forms a key basis for Sinkula et al.'s (1999) market-based organisational learning framework. Boxall and Purcell's (2003) distinction between business strategy and competitive strategy is useful here, as it is an organisation's competitive strategy that shapes its business strategy. Further, Porter's (1985) classification of generic competitive strategies (cost leadership, differentiation and focus) and Boxall's (2003) classification of market characteristics, 
competitive dynamics and HR strategy are starting points for understanding the strategic choices that organisations make in their chosen area of service or product markets. Using Boxall's (2003) classification, firms can compete in mass-service markets, a mix of massservice markets and slight value-added segments of the market, and/or highly differentiated service markets. The production function, HR strategy, and competitive dynamics that typify each of these three market segments vary with an organisation's strategic milieu and the competitive strategy(ies) it follows (see Figure 7, Chapter 3).

Following the within-case analysis, it is clear that these market segments are not mutually exclusive. Competition was based on price and quality. Various combinations of one or all three market segments and competitive strategies were found to exist in the organisations studied. This diversity is also reflected in the organisation of a firm's production function. However, the relationship between an organisation's competitive strategy and volume of training is not straightforward; among other factors, it is also influenced by the complexity of its products and services (Green et al., 2003; Mason, 2004, 2005). Using Boxall's typology the relationship between competitive strategy and training volume is depicted in Figure 26.

\section{Mass-Service Markets (MP)}

Organisation D is catering to mix of mass-service (medical transcription centre) and low to medium value-added service markets (F\&A back office). Both services followed a lowcost producer strategy. MT has a high volume of transactions, low employee discretion, minimal training provision, and a highly Taylorist work organisation. F\&A service line, which attracts higher billing rates, has an informal team-based structure, allows medium discretion, and offers a low volume of training.

\section{Slightly Differentiated Markets (SD)}

Organisations I and C, although competing in a slightly differentiated market, follow a blend of cost minimisation and differentiation strategies. Further, their relatively simple process/product portfolios required limited investment in training volume. Additionally, both organisations have a highly constrained and low-discretion work environment, typical of a Taylorist work setting, with training provision in a narrow range, one that focuses exclusively on job-specific skills. Relative to I and C, Organisations B, H, and J have medium to high levels of process complexity and cater to a slightly differentiated market (see Figure 27). In these case organisations, the average level of skills to perform 
the jobs is higher. People were hired with higher qualifications and skills, and, because of the mismatch between the educational curriculum, evolving skills infrastructure, and the nature of the work received, additional training was also needed to close the skills gap.

\section{Highly Differentiated Markets (HD)}

Organisations A, E, F and G have strong market shares in their respective services and product markets. Leveraging their QMS capabilities, Organisations A and F are following a cost leadership and differentiation strategy. The nature of work undertaken by these organisations ranges from simple to highly complex and involves delivering a range of business and technology solutions to numerous industry sectors. High process and project complexity require additional training (Green et al., 2003; Mason, 2004, 2005) on a continuum from simple to complex, albeit in a narrow range, through effective deployment of its quality management capabilities and reengineering jobs.

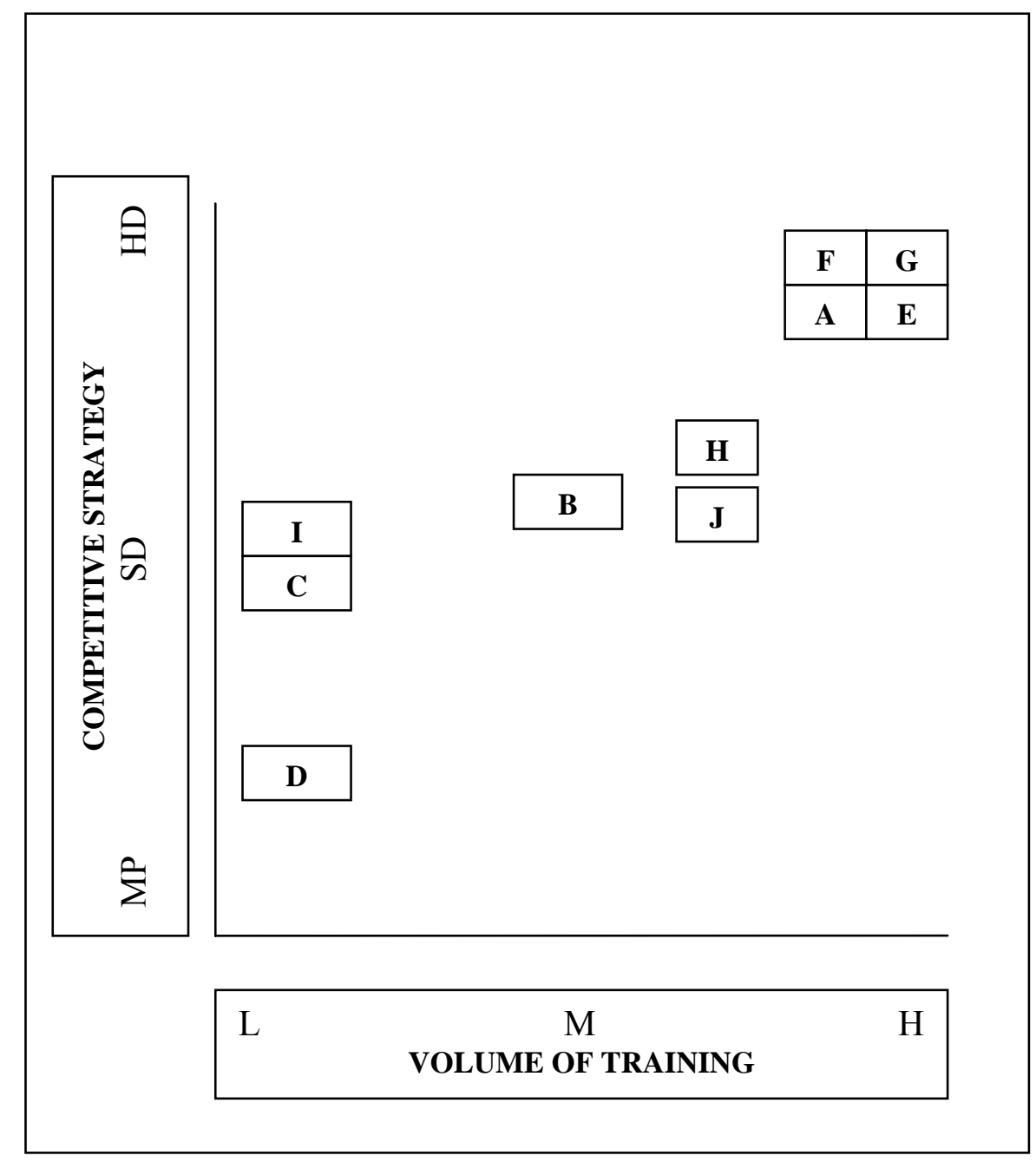

Figure 26. Competitive strategy and volume of training. 
Table 17

Factors Influencing Training

\begin{tabular}{|c|c|c|c|c|c|c|c|c|c|c|c|}
\hline S.No. & Main factors and attributes & A & B & C & D & $\mathbf{E}$ & $\mathbf{F}$ & G & $\mathbf{H}$ & I & $\mathbf{J}$ \\
\hline 1 & $\begin{array}{l}\text { Competitive strategy } \\
\mathrm{MP}=\text { Mass production } \\
\mathrm{SD}=\text { Slightly differentiated } \\
\mathrm{HD}=\text { Highly differentiated }\end{array}$ & HD & $\mathrm{SD}$ & SD & $\begin{array}{l}\text { MP\& } \\
\text { SD }\end{array}$ & HD & HD & HD & SD & SD & SD \\
\hline 2 & $\begin{array}{l}\text { Workplace change } \\
\text {-structural } \\
\text {-ownership } \\
\text {-technological } \\
\end{array}$ & $\begin{array}{l}\mathrm{H} \\
\mathrm{H} \\
\mathrm{M}\end{array}$ & $\begin{array}{l}\mathrm{H} \\
\mathrm{N} \\
\mathrm{H} \\
\end{array}$ & $\begin{array}{l}\mathrm{M} \\
\mathrm{H} \\
\mathrm{H}\end{array}$ & $\begin{array}{l}\mathrm{H} \\
\mathrm{L} \\
\mathrm{M}\end{array}$ & $\begin{array}{l}\mathrm{H} \\
\mathrm{H} \\
\mathrm{H}\end{array}$ & $\begin{array}{l}\mathrm{H} \\
\mathrm{M} \\
\mathrm{H}\end{array}$ & $\begin{array}{l}\mathrm{H} \\
\mathrm{H} \\
\mathrm{H} \\
\end{array}$ & $\begin{array}{l}\mathrm{M} \\
\mathrm{M} \\
\mathrm{H}\end{array}$ & $\begin{array}{l}\mathrm{L} \\
\mathrm{H} \\
\mathrm{H}\end{array}$ & $\begin{array}{l}\mathrm{H} \\
\mathrm{L} \\
\mathrm{H}\end{array}$ \\
\hline 3 & $\begin{array}{l}\text { Market orientation } \\
\text {-information sensing } \\
\text {-information dissemination } \\
\text {-organisational response }\end{array}$ & $\begin{array}{l}\mathrm{H} \\
\mathrm{H} \\
\mathrm{H}\end{array}$ & $\begin{array}{l}\mathrm{M}-\mathrm{H} \\
\mathrm{H} \\
\mathrm{M}\end{array}$ & $\begin{array}{l}\mathrm{H} \\
\mathrm{H} \\
\mathrm{M}\end{array}$ & $\begin{array}{l}\mathrm{M}-\mathrm{H} \\
\mathrm{M} \\
\mathrm{M}\end{array}$ & $\begin{array}{l}\mathrm{M}-\mathrm{H} \\
\mathrm{H} \\
\mathrm{H}\end{array}$ & $\begin{array}{l}\mathrm{H} \\
\mathrm{H} \\
\mathrm{H}\end{array}$ & $\begin{array}{l}\text { M } \\
\text { M-H } \\
\text { M-L }\end{array}$ & $\begin{array}{l}\mathrm{H} \\
\mathrm{M}-\mathrm{L} \\
\mathrm{M}-\mathrm{H}\end{array}$ & $\begin{array}{l}\mathrm{M} \\
\mathrm{H} \\
\mathrm{M}-\mathrm{H}\end{array}$ & $\begin{array}{l}\text { M } \\
\text { M-H } \\
\text { M-H }\end{array}$ \\
\hline 4 & $\begin{array}{l}\text { Learning orientation } \\
\text {-commitment to learning } \\
\text {-open-mindedness } \\
\text {-shared organisational vision } \\
\end{array}$ & $\begin{array}{l}\mathrm{H} \\
\mathrm{H} \\
\mathrm{H} \\
\end{array}$ & $\begin{array}{l}\mathrm{H} \\
\mathrm{M} \\
\mathrm{M}\end{array}$ & $\begin{array}{l}\mathrm{M} \\
\mathrm{H} \\
\mathrm{M}\end{array}$ & $\begin{array}{l}\text { M-L } \\
\text { M-L } \\
\text { M-L }\end{array}$ & $\begin{array}{l}\mathrm{H} \\
\mathrm{M} \\
\mathrm{H} \\
\end{array}$ & $\begin{array}{l}\mathrm{H} \\
\mathrm{M}-\mathrm{H} \\
\mathrm{H}\end{array}$ & $\begin{array}{l}\mathrm{H} \\
\mathrm{H} \\
\mathrm{H} \\
\end{array}$ & $\begin{array}{l}\text { M-L } \\
\text { M-L } \\
\text { M-L }\end{array}$ & $\begin{array}{l}\text { L } \\
\text { L-M } \\
\text { L-M }\end{array}$ & $\begin{array}{l}\mathrm{M}-\mathrm{L} \\
\mathrm{M} \\
\mathrm{M} \\
\end{array}$ \\
\hline 5 & $\begin{array}{l}\text { Quality management systems } \\
\text { - commitment \& sharing information } \\
\text {-continuous improvement } \\
\text {-teamwork }\end{array}$ & $\begin{array}{l}\mathrm{H} \\
\mathrm{H} \\
\mathrm{H}\end{array}$ & $\begin{array}{l}\text { M-H } \\
\text { M-H } \\
\text { M }\end{array}$ & $\begin{array}{l}\mathrm{M} \\
\mathrm{H} \\
\mathrm{M}\end{array}$ & $\begin{array}{l}\mathrm{M} \\
\mathrm{M} \\
\mathrm{H}\end{array}$ & $\begin{array}{l}\mathrm{H} \\
\mathrm{H} \\
\mathrm{M}-\mathrm{H}\end{array}$ & $\begin{array}{l}\mathrm{H} \\
\mathrm{H} \\
\mathrm{M}-\mathrm{H}\end{array}$ & $\begin{array}{l}\mathrm{H} \\
\mathrm{H} \\
\mathrm{H}\end{array}$ & $\begin{array}{l}\mathrm{M} \\
\mathrm{M}-\mathrm{H} \\
\mathrm{H}\end{array}$ & $\begin{array}{l}\text { M } \\
\mathrm{M} \\
\mathrm{M}-\mathrm{L}\end{array}$ & $\begin{array}{l}\mathrm{M} \\
\mathrm{M} \\
\mathrm{H}\end{array}$ \\
\hline 6 & Employee turnover & $\begin{array}{l}\mathrm{H} \text { to } \\
\mathrm{M} \text { to } \mathrm{L}\end{array}$ & $\mathrm{H}$ and $\mathrm{L}$ & $\begin{array}{l}\mathrm{H} \& \\
\mathrm{M} \text { to L }\end{array}$ & $\mathrm{L}$ & $\mathrm{H}$ & $\mathrm{H}$ & $\mathrm{L}-\mathrm{M}$ & $\mathrm{M}-\mathrm{L}$ & $\mathrm{H}$ & $\mathrm{M}$ \\
\hline 7 & $\begin{array}{l}\text { Enterprise size } \\
\text { Complexity } \\
\text { Ownership }\end{array}$ & $\begin{array}{l}\mathrm{H} \\
\mathrm{M}-\mathrm{H} \\
\mathrm{MNC} \\
\end{array}$ & $\begin{array}{l}\text { L-M } \\
\text { M-H } \\
\text { Indian }\end{array}$ & $\begin{array}{l}\mathrm{M} \\
\mathrm{M} \\
\mathrm{JV}(\text { Indian})\end{array}$ & $\begin{array}{l}\mathrm{L} \\
\mathrm{M}-\mathrm{L} \\
\text { Indian }\end{array}$ & $\begin{array}{l}\mathrm{H} \\
\mathrm{H} \\
\mathrm{MNC} \\
\end{array}$ & $\begin{array}{l}\mathrm{H} \\
\mathrm{H} \\
\text { Indian } \\
\end{array}$ & $\begin{array}{l}\mathrm{H} \\
\mathrm{H} \\
\mathrm{MNC}\end{array}$ & $\begin{array}{l}\mathrm{M} \\
\mathrm{M} \\
\text { Indian }\end{array}$ & $\begin{array}{l}\mathrm{M} \\
\mathrm{M} \\
\mathrm{MNC} \\
\end{array}$ & $\begin{array}{l}\mathrm{L} \\
\mathrm{M}-\mathrm{H} \\
\mathrm{MNC}\end{array}$ \\
\hline 8 & Temporal dimension & $\mathrm{H}$ & $\mathrm{H}$ & $\mathrm{H}$ & $\mathrm{L}$ & $\mathrm{M}-\mathrm{L}$ & $\mathrm{M}-\mathrm{H}$ & $\mathrm{M}-\mathrm{L}$ & $\mathrm{M}-\mathrm{L}$ & $\mathrm{M}-\mathrm{L}$ & $\mathrm{M}-\mathrm{L}$ \\
\hline 9 & Geographical dimension & $\mathrm{H}$ & $\mathrm{H}$ & $\mathrm{M}$ & $\mathrm{M}-\mathrm{L}$ & $\mathrm{L}$ & $\mathrm{L}$ & $\mathrm{L}$ & $\mathrm{M}-\mathrm{L}$ & $\mathrm{N}$ & $\mathrm{L}$ \\
\hline 10 & $\begin{array}{l}\text { Strategic HRM approach } \\
\text {-rewards and performance } \\
\text { management systems } \\
\text {-career planning } \\
\text {-recruitment skills level \&operating ro } \\
\text {-use of non-standard employment }\end{array}$ & $\begin{array}{l}\mathrm{H} \\
\mathrm{H} \\
\mathrm{H} \\
\mathrm{L}\end{array}$ & $\begin{array}{l}\mathrm{H} \\
\mathrm{M} \\
\mathrm{H} \\
\mathrm{N}\end{array}$ & $\begin{array}{l}\mathrm{H} \\
\mathrm{H} \\
\mathrm{H} \\
\mathrm{L}\end{array}$ & $\begin{array}{l}\mathrm{M}-\mathrm{H} \\
\mathrm{N} \\
\mathrm{H} \\
\mathrm{L}\end{array}$ & $\begin{array}{l}\mathrm{H} \\
\mathrm{H} \\
\mathrm{H} \\
\mathrm{L}\end{array}$ & $\begin{array}{l}\mathrm{H} \\
\mathrm{H} \\
\mathrm{H} \\
\mathrm{L}\end{array}$ & $\begin{array}{l}\mathrm{H} \\
\mathrm{M}-\mathrm{H} \\
\mathrm{M} \\
\mathrm{L}\end{array}$ & $\begin{array}{l}\text { M } \\
\text { M-L } \\
\text { M-H } \\
\text { M-L }\end{array}$ & $\begin{array}{l}\mathrm{H} \\
\mathrm{L} \\
\mathrm{H} \\
\mathrm{N}\end{array}$ & $\begin{array}{l}\text { M-LM- } \\
\text { LHL }\end{array}$ \\
\hline
\end{tabular}




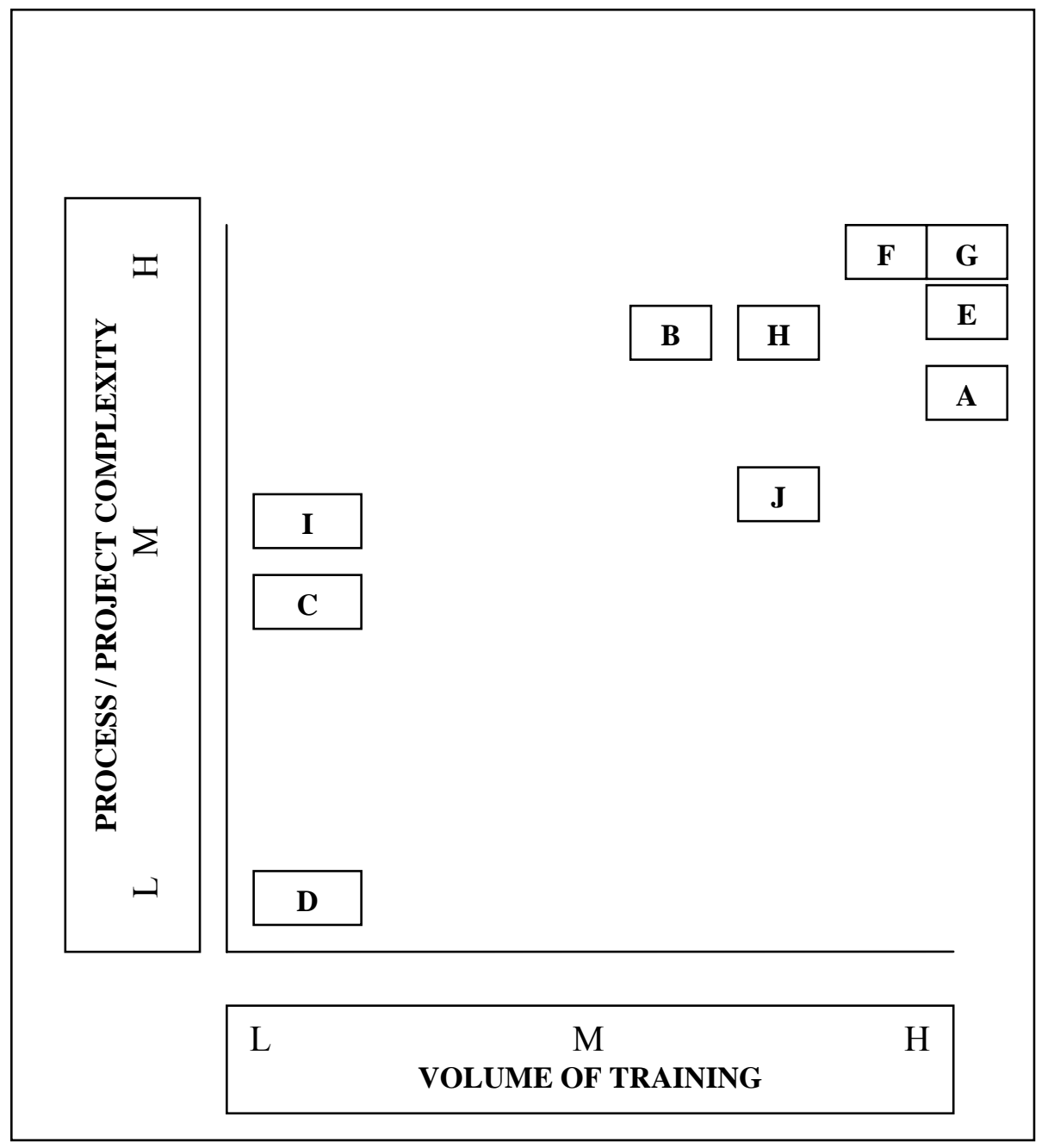

Figure 27. Process or project complexity and volume of training.

Following a flexible specialisation approach (Piore \& Sabel, 1984), all four organisations invested deliberately, in the early stages of their evolution, to develop strong training infrastructures and quality management systems, and implement complementary HRM practices (for example, by recruiting from campuses and, strategically developing and deploying people to different projects) that allowed them firms to compete effectively on low cost and high quality.

However, Organisations E and G have not fully realised their cost leadership and QMS abilities for their production function in India. To some extent, this complacency is due to the global geopolitical uncertainty about the future of offshoring and to their inability to fully transfer their parent organisations' path dependencies and social complexities to the Indian cultural settings. While competitive strategy sets the tone for operating in certain markets, it is not sufficient to fully explain the business strategy choices (HR, operational, marketing, and so on) that firms make to maintain high levels of performance. 
From a training and, more broadly, from an HR perspective, once an organisation has decided on the competitive market it wants to operate in, it has to exercise a choice whether to "make" (develop) or "buy" (acquire) resources from the external labour market to successfully deliver its products and services. While such a choice sets the tone for investment in training, the exact nature and extent of training is shaped by a number of internal and external influences in an organisation's strategic milieu. These influences are analysed below.

\section{HR Strategy}

An organisation's decision to "make" or "buy" resources has to support its cost leadership, or differentiation, or a mix of such strategies. Such decisions are also influenced by the nature of the markets in which organisations are operating. Although the Indian labour market is known to have deep pockets of qualified and skilled engineers and graduates, a closer analysis of their employability by the ITSS and ITeS/BPO sectors is still a concern (NTITSD, 2003; Narayanan \& Neethi, 2005). Moreover, the high rate of technology skills obsolescence and the dynamic nature of processes and projects necessitate an ongoing demand for training and upskilling.

As noted in chapter 2, post 2000, the competition in factor markets intensified. These changes in the strategic milieus of firms resulted in rising wage costs and exploration of new business models and hiring strategies. Consequently, some organisations invested in building training capabilities and others did not. The case organisations that did invest significantly in training also followed a combination of cost leadership and differentiation strategies in more than one facet of their service delivery. In the evolution of the Indian IT sector, the early movers or innovators (Wernerfelt, 1984) had foreseen the issue of cost and availability of skilled resources and thus invested heavily in developing their learning and development infrastructures.

As these early movers grew in size and scope, they were able to deliver an overall cost and quality advantage to their clients. In their business model, training is viewed as a strategic capability and, in most cases, has been inextricably intertwined with operations, rather than being an integral part of a firm's HRM infrastructure. This nexus between training and operations differs significantly from the extant literature, which traditionally 
sees training as an integral part of HRM and a litmus test for the presence of a strategic approach to HRM.

Training was found to be separate both structurally and functionally from the case organisations' HRM infrastructure. The only link to HR was where an organisation's shared HR services were managing the logistical and planning arrangements of training (room booking, trainee booking and follow-ups, cost centre charging, and evaluation, or developing a skills database and inventories for redeployment purposes). Even the training needs analysis, design, and development was usually undertaken by specialist training resources, and sometimes with the help of their quality management resources.

Nevertheless, there were significant linkages noted between HR and training, especially with focused recruitment strategies and in some large organisations (Organisation A, E, F, and $\mathrm{G}$ ), where there was a need to provide employees with avenues for career development. Here, too, retention and talent management were considered from a cost leadership angle. Figure 28 illustrates the relationship between training volume and recruitment strategy. To enable the realisation of Organisations A, E, F and G's integrated cost leadership strategy in human resources, they had a well-developed graduate training programme and, relative to their total hiring, relied more on recruitment of graduates. Consequently, although their overall investment in training volume was high, their ability to contain the costs was successful because the majority of the employees were graduates with low experience and wage costs. Similarly, Organisations C, D, I, and to a lesser extent $\mathrm{J}$, relied almost exclusively on experienced staff, had a low volume of training. Organisations $\mathrm{B}$ and $\mathrm{H}$ relied on a combination of experienced and recent college graduates and provided medium levels of training.

Despite the above strategy, there were notable differences in the shaping of training, even within the larger enterprises. For example, Organisations E and G, although providing high volumes of training, for reasons explained earlier did not have an internal training infrastructure. Similarly, Organisations $\mathrm{J}$ and $\mathrm{H}$ relied more on external training than other smaller organisations (C, D and I). The fact that Organisation $\mathrm{J}$ is a small ODC, and I, being in a product environment explains their reliance on high and low external training, respectively. Likewise, Organisation C's niche industry sector (real estate) and D's unique distributed vendor-based model decreased the need for any external training. 
To explain the dynamics of training decision making at a business level, it is critical to understand the differences among organisations' values and its behaviours, which

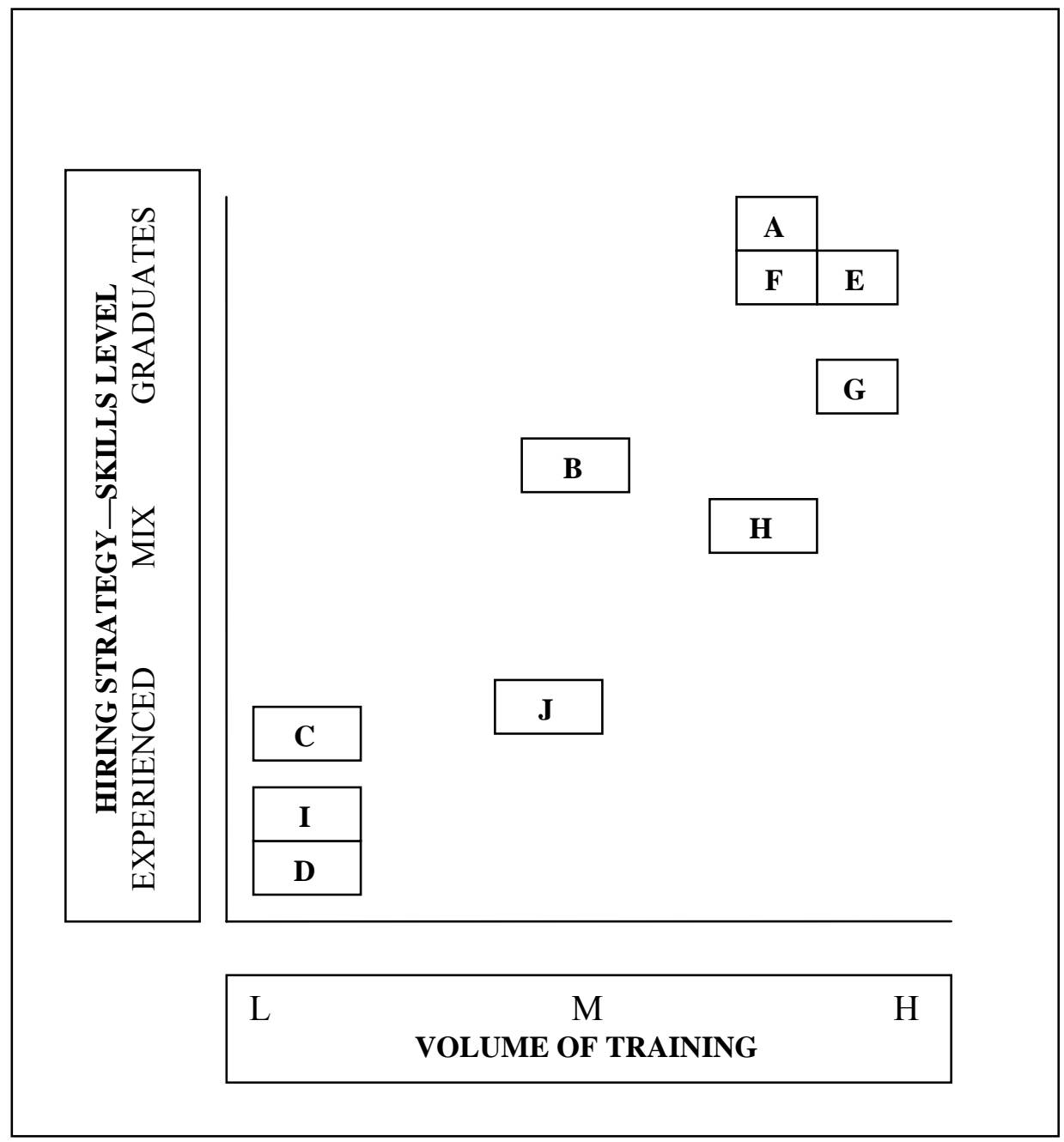

Figure 28. Recruitment strategy and volume of training.

are demonstrated by the strategic choices it makes and leads to the development of certain organisational capabilities. Further, it is the interaction between various factors that ultimately shapes the final nature and extent of training. On the assumption that values drive behaviours and actions, the following section analyses the role of an organisation's QMS and other capabilities such as learning orientation (knowledge-questioning values) and market orientation (knowledge-producing behaviours) using Sinkula et al.'s (1997) market-based organisational learning framework. In a dynamic services environment, development of these capabilities is critical, but not sufficient, as subsequent analysis will make clear. 


\section{Organisational Values, Behaviours, and Capabilities}

By use of Sinkula et al.'s (1997) market-based organisation learning framework, similarities and differences in the case organisations' MO and LO abilities are compared and differences in training provision analysed. Differences exist between firms' MO in a services and product environment. What is not clear is why firms have different levels of HRM, MO and LO capabilities. What is the enabler? What is the key variable? How can high MO and LO be developed? The reasons for these differences will be analysed and explained later in the discussion on quality management systems.

\section{Market Orientation (MO) and Client Specifications}

All ITeS/BPO firms (Organisations A, B, C, and D) noted client specifications as an important factor in influencing their training needs. Training was often organised around the key deliverables agreed with a client in their service level agreements. Thus, the role of information sensing and disseminating is critical in training needs identification for the delivery teams. However, for IT services and product firms, the impact of client specification on training, although evident, was not so strong. The reasoning behind this difference is the generic nature of technology and software applications: it cuts across borders. Client specifications impacted on training provision in ITSS firms only when the clients were using certain proprietary tools and applications, which have very limited use in the wider IT market. Such specialist tools and applications were used particularly by clients of Organisations E, F, and I. Understanding the domain and clients' business logic also required specialist training.

In an ITSS and ITeS/BPO sector context, firms can be grouped into shrink-wrap (product) firms (Organisation $G$ and I), and project or processes (services) firms (Organsiations A, B, C, D, E, F, H and J). Some of the larger firms, like Organisation E and F, offer end-to-end ITSS and ITeS/BPO solutions. Extant literature suggests that product environment firms typically have lower levels of MO than firms in a services environment (Cano et al., 2004). Support was weak for this finding as both product environment firms had to deliver customisation services (Organisation I because of customisation requests from hospitals) and redesign their products in line with the new customer demands (Organisation G's move for platformisation). This reasoning may explain the relatively medium to high levels of MO found in case organisations. 
Because of the collaborative nature of design and development work between teams in India and overseas, clients' information sensing is usually geographically distributed. Thus, this analysis provides an assessment of the MO capabilities of sites only in India. Where possible, insights about the parent firms' MO capabilities were also noted in the within-case analysis. The MO level referred to here is the extent to which a third-party service provider or a wholly owned offshore development centre facility senses information from the clients (external or parent organisation) about a given project or process, disseminates it to the delivery team in India, and frames appropriate operational responses.

To this end, all of these case organisations can be viewed as service firms with varying levels of MO and training volume. For example, Organisations C, I and D have medium to high levels of MO and low training volume. Although it is understandable for Organisation I (product environment) and to some extent D (distributed vendor-based model) to have a low training volume, Organisation C's low training volume can mostly be attributed to its relatively simple processes. Overall, there is no set pattern for a relationship between an organisation's MO and its training volume (see Figure 29), which suggests that, on its own, MO does not influence training volume. The extant literature suggests that high LO is helpful in developing an organisation's MO (Sinkula et al., 1997).

\section{Learning Orientation (LO)}

An organisation's commitment to learning, open-mindedness to new learning and information, and ability to develop a shared vision constitute its LO (Sinkula et al., 1997). LO represents an organisation's knowledge-questioning values. Organisations A, E, F and $\mathrm{G}$ have medium to high levels of LO and a high level of training volume (see Figure 30). The above organisations also demonstrate double-loop learning (Argyris \& Schon, 1978) in their daily routines, and have well-developed training infrastructures and informationsharing networks. In most cases, it is the nature of work that enables organisations to undertake critical thinking and thus to challenge clients and their own business assumptions. For example, Organisation G's high focus on technology leadership in the high-end semiconductor market requires its research, development, and design team in India to engage in a high degree of open-mindedness. 


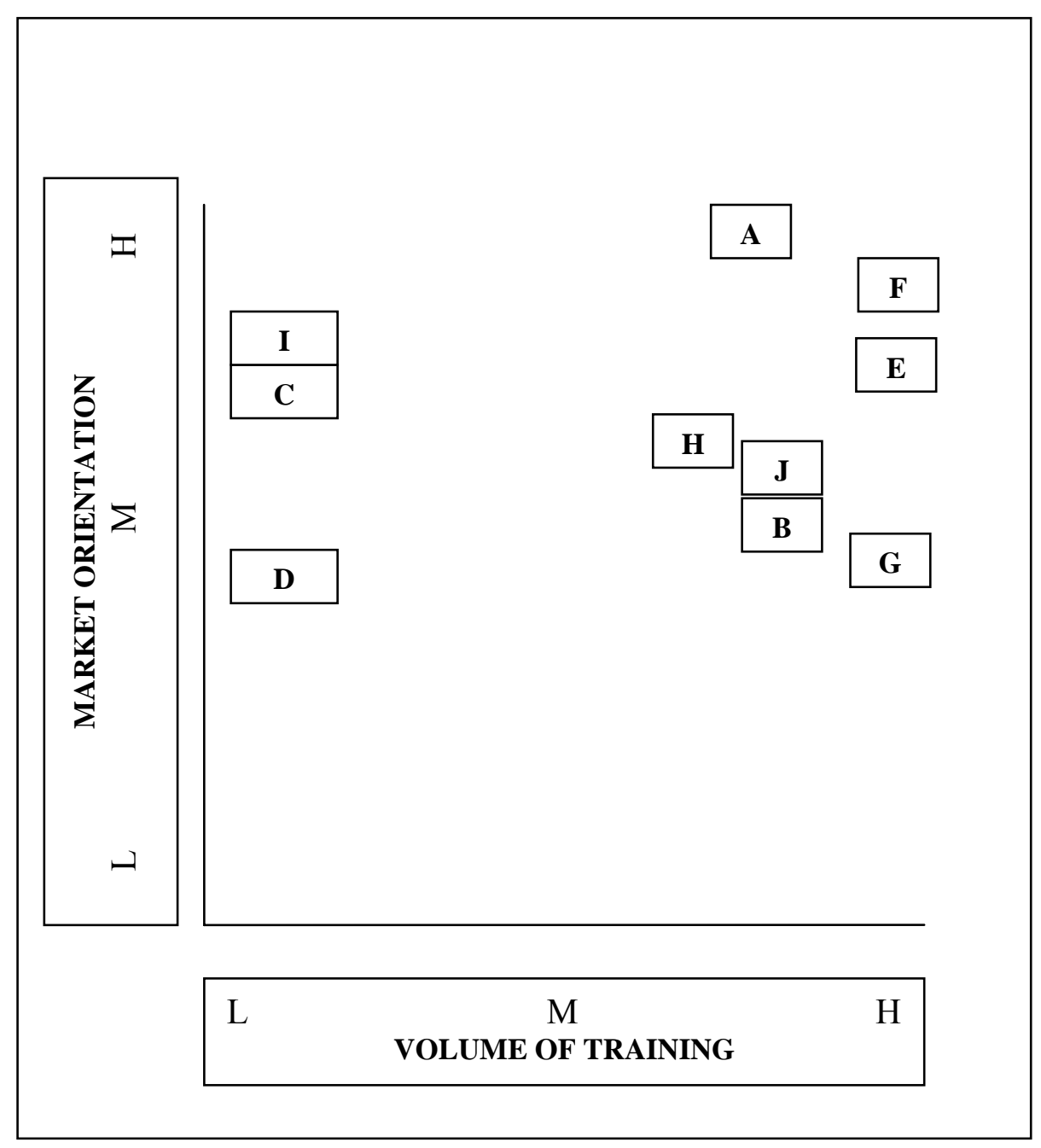

Figure 29. Market orientation and volume of training.

It is important to note here that even if an organisation has high levels of learning commitment and well-developed platforms for promoting a shared vision or key values, its overall LO can be adversely affected by its medium to low LO open-mindedness: its ability to critically challenge its clients' assumptions. This does not suggest that only organisations with high work complexity can challenge their clients' and their own business assumptions or firms with low complexity do not engage in open-mindedness. Instead, the opportunities to engage in such learning are limited in certain circumstances. Similarly, the ability to develop a shared vision is also critical to infuse values.

A low level of open-mindedness can be due to many reasons. First, it may result from a clients' unwillingness to accept high quality solutions suggested by the service provider. Second, the service provider may be inexperienced in a given process or domain and lack the capability to engage in higher order learning. Finally, if there is limited economic 
return for engaging in higher level learning processes for both the organisation and the client, especially where the nature of work is at the low end, there is no incentive for engaging in higher order learning. In all the above cases, the overall learning orientation is influenced by the strength of an organisation's quality management capabilities.

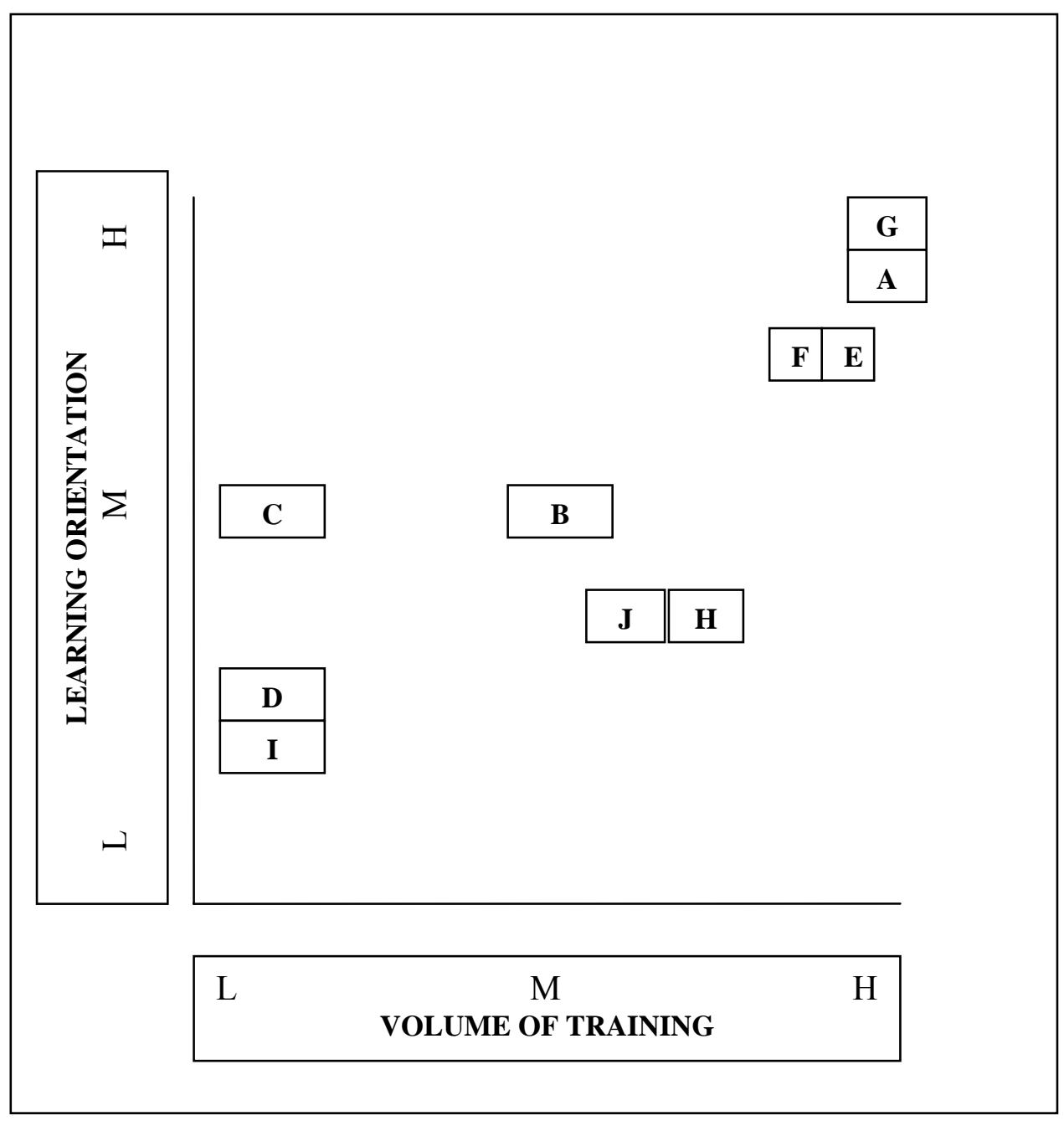

Figure 30. Learning orientation and training.

Thus, although Organisation D and I have relatively simple work processes, their limited internal capabilities (such as quality management systems), their limited ability to service clients' needs, and the nature of their work organisation appear to explain the relatively low volume of training. Likewise, Organisations B, H and J's medium to low LO and medium to high training volume correspond to their evolving organisational capabilities. The case findings suggest that an organisation's quality management system is the critical and unexplored link in the development of an organisation's LO and MO. This finding is a departure from the market-based organisational learning framework (Sinkula et al., 1997), which argues that strong LO influences MO and that the interaction between LO 
and MO has a synergistic effect on an organisation's performance. The following analysis demonstrates how an organisation's quality management system, which, like LO and MO, exists on a continuum, helps in developing its LO, MO and other organisational capabilities, which ultimately have an impact on training provision.

\section{Quality Management Systems (QMS)}

As noted in Chapter 3, India's IT sector has adopted numerous quality management standards, such as ISO 9000, CMM (Levels 1-5), CMMI (Levels 1-5), COPC, Six Sigma, Lean Six-Sigma, and so on (Kumar, 2001; NASSCOM, 2006). Further, the literature on training drivers (Dawe, 2003; Ridoutt et al., 2002; Smith \& Hayton, 1999; Smith et al., 2004) and HPWPs, which often includes quality management systems as one of the key work practices in the bundle of practices (Ashton \& Sung, 2002; 2005; Snell \& Dean, 1992; Osterman, 1995), suggests that investment in quality is a strong driver for training. What is not adequately explained in these studies is how and why quality drives training. Extant literature suggests that adoption of quality accreditation banners such as ISO 9000 requires investment in some mandated training as part of the accreditation requirements. While this is true also in the Indian experience, there is a lot more about how and why that needs further exploration and analysis.

At this stage of the discussion, it is important to note that, like other organisational capabilities, an organisation's quality management capability exists on a continuum. The nature of these capabilities and the extent to which they can be implemented are not the focus of this study. However, as the within-case and this analysis suggest, there are strong linkages between an organisation's competitive strategy and the strength of its QMS, as the latter helps in defining the competitive position a firm may take (Belohlav, 1993; Morgan \& Piercy, 1996; Prajogo, 2007). Such investments do not imply exclusively that only formal forms of quality accreditation systems are worthy of attention. Following Prajogo and Brown (2004), it is more the TQM principles that have been developed and the extent to which these can be applied and embedded in an organisation's production function (see for example, Organisation $G$ ) that matter and form the focus of ensuing analysis. Figure 31 shows the relationship between an organisation's QMS and its training volume. 
Following Reed at al. (2000), TQM content can focus on internal and external dimensions of product or service quality. While the former focuses on process efficiencies and effectively contributes to an organisation's cost leadership strategy, the latter focuses on developing a basis for differentiation and improving customer satisfaction. Reed et al. further argue that although TQM content may offer competitive advantage, it is the process of how an organisation deploys its QMS that can create sustained competitive advantage.

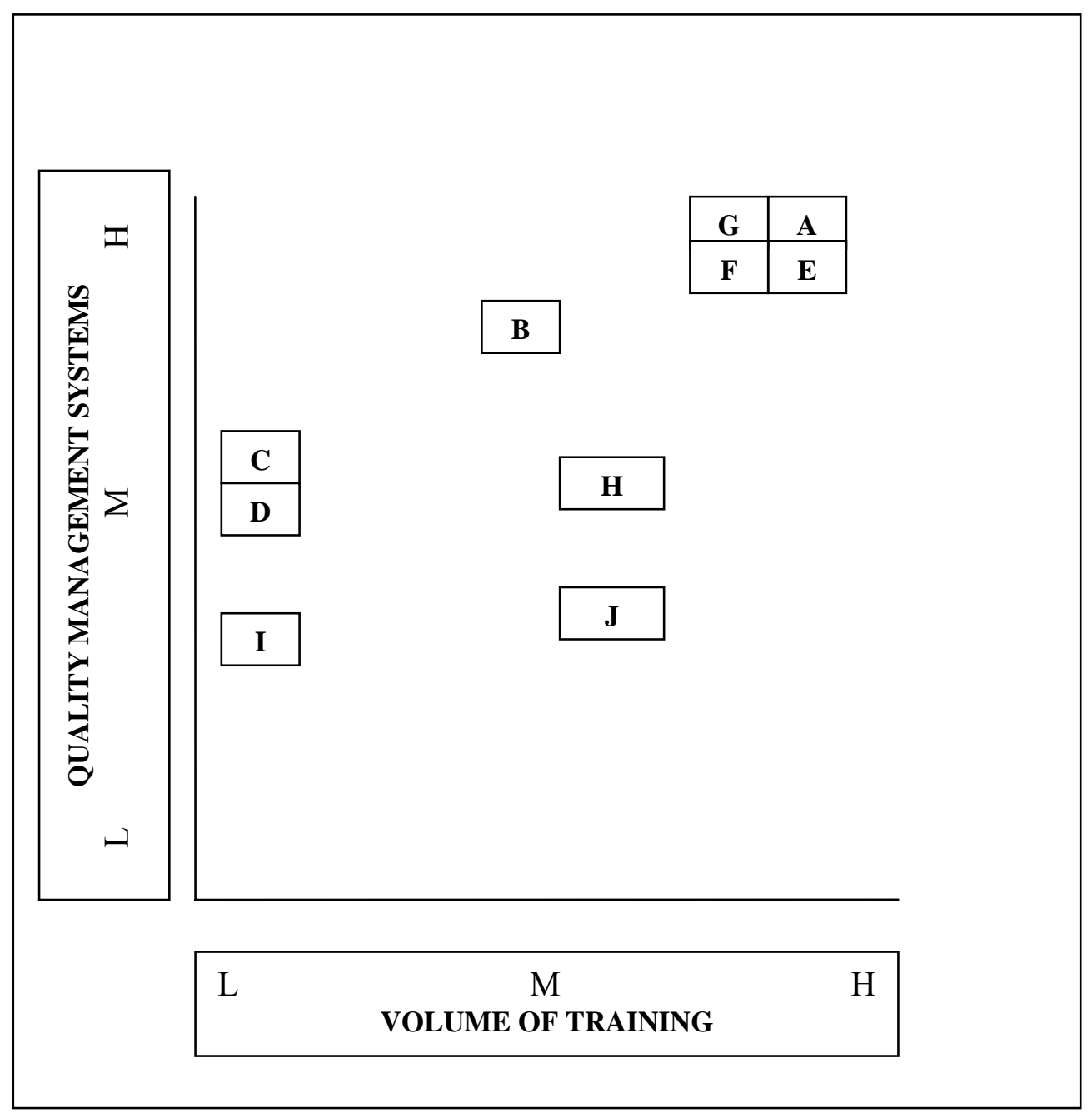

Figure 31. Quality management systems and training.

QMS impacts on MO and LO capability development at different stages. First, using QMS tools and methodologies, an organisation can develop strong internal standards of performance, which help in interpreting information and developing organisational memory (not covered in Sinkula et al.'s (1997) empirical framework). These standards guide the sensing of specific information from clients for proposing solutions, which may contribute to high project/process efficiency levels. Second, when the information is disseminated, it is critical to select and disseminate those metrics that are critical to 
quality or performance of the process/project, thus enabling project teams to focus on specific areas of delivery response. Teamwork enables dissemination and inter-functional coordination in framing appropriate responses.

Similarly, for LO, if an organisation has well-developed standards of performance and processes for various business processes, its ability to develop a shared vision for such metrics is much easier. Unclear performance expectations and confusion over what is doable creates problems in training need analysis and consequently affect its commitment to learning. Finally, an organisation cannot successfully demonstrate open-mindedness, or its ability to critically challenge its own and clients' business processes if its internal benchmarks or standards of performance are lacking.

Organisations A, E, F and G have all invested heavily in their quality management infrastructure. Although an accreditation standard sets the tone for different levels of quality standards, it does not prescribe exactly how an organisation can apply these standards to their own work circumstances. For example, although Six Sigma principles have existed for nearly three decades (Fazzari \& Levitt, 2008; Breyfogle, 1999), how organisations develop and deploy them is critical in providing competitive benefits and advantage (see for example, Appendix 5). Such benefits have to be significant. Organisations would not be making sustained investments of millions of dollars or rupees in building a quality management infrastructure merely for the sake of improved marketsignalling. For example, Organisation A's capability in deploying SS techniques to all its business processes evolved over a period of time and was partly influenced by its parent organisation's beliefs. Organisation A was able to implement them in India (with more than 200 SS-certified professionals) because of its evolving capabilities and the relatively low labour cost of implementing such practices in India.

Similarly, Organisation F invested heavily in quality management standards such as Malcolm Baldridge, ISO, SS, and CMM more than a decade ago. There was vision in the leadership of both Organisations A and F and they realised the critical role investment in quality can play in increasing predictability of service delivery and, consequently, in efficient and effective large-scale people management, especially if the work covers a diverse range of projects. Quality management systems enable predictable standards of delivery (standardisation) by following established internal benchmarks and standards, 
and streamline continuous improvement and cost reduction efforts by implementing statistical process control and SS methodologies.

While it does not follow any popular quality management accreditation, Organisation E has, over a long period of time, established its own standards relevant to the semiconductor industry. These standards are unique to the organisation and a highly customised to its business and product development processes. Thus, although there is a high level of standardisation, these standards are difficult to replicate by others, because they are highly customised to the organisation's internal business and technology processes.

Organisation B has committed resources to quality management accreditations. However, because it is a relatively new organisation and does not have well developed internal quality standards and information-sharing mechanisms for the various stages of its process life cycle, its ability to bring about improvements is limited. Consequently, the need to provide training is less. The QMS of Organisations C, D, and $\mathrm{H}$ are at medium level and, as their portfolio of services is relatively simple and process complexity is medium to low, the volume of training is also low. Finally, Organisation $\mathrm{J}$ has a medium to high training volume since it is in the early stages of its evolution and a lot of new learning is being consolidated through trial and error. What is interesting to note from the above analysis is that firms operating in a product environment (Organisations G, I, and to a lesser extent, E) have not adopted popular quality management accreditations. The reasons are their perceived product superiority and their strong internal standards (Organisations G, E, and to a lesser extent I) for software product development processes. These standards have been refined over a long period of time. Only when these firms enter into the services market do they feel the need to look at additional forms of quality management standards, for market signalling as well as for delivering quality from a customer's viewpoint. This trend was evident in Organisations E and G.

It follows from the above analysis that it is the ability to apply and deploy quality management resources that creates differences in organisational capabilities and training. The earlier relationships between LO and MO (Sinkula et al., 1997) are better explained by the strength of an organisation's QMS. Organisations that did not possess strong QMS also had relatively weak LO and MO abilities. The presence of strong quality management systems also explains a high level of training formalisation. 


\section{Other Factors}

The following section analyses the impact of various other factors that influence training provision and shows how workplace change and work organisation interact with an organisation's LO, MO, and QMS capabilities.

\section{Workplace Change}

Researchers from Australia, the US and the UK (Ashton \& Sung, 2002; Cappelli, 1994; Green et al., 2003; Osterman, 1995; Smith et al., 2003; Ridoutt et al., 2002) and studies from other OECD countries (OECD/CERI, 1986, 1988) found workplace changetechnological or structural - and the implementation of new management practices or HPWPs (such as learning organisation, team work, TQM, lean production, and business process reengineering) to have a profound impact on training provision. Overall, this study confirms the above findings that technological and structural change influences training.

The exceptions were Organisations C, D, and I, where there were medium to high levels of structural and technological changes but the impact on training volume was limited. Organisation C's relatively simple portfolio and stabilised business processes reduced the overall demand for training. Although Organisation D went through a major restructure, its resultant need for training was low as it transferred its training costs to its network of external medical transcription service vendors in different parts of India. Organisation I wanted to move to a newer technology platform (.Net) for its single product portfolio. The overall impact on training was not high because only a select group of employees were involved in such training. This suggests that if the technical change impacts on the wider work organisation rather than on a certain process or a certain group of employees, training demand is high.

\section{Work Organisation}

Nature of work typified digital Taylorism, especially in voice and low-end transaction processing of non-voice services. Organisations that followed a differentiated competitive strategy followed a mix of Taylorist and digital Taylorist structures. Depending on the organisation's ability to simplify processes and organise roles based on the simple to 
complex tasks continuum, a Fordist or a variant of a post-Fordist work organisation was evident. Such work organisation and task continuum were not just limited to ITeS/BPO firms; they were also evident in ITSS firms, where the Waterfall Model or similar software development lifecycle models were followed. Firms that had a better understanding of the exact nature of tasks to be performed for different roles/competencies at various stages of a process or project's lifecycle also had well developed QMS. QMS help in the simplification and standardisation of tasks and activities and in adopting a "best practice" approach for service delivery.

The linkages between an organisation's work design, QMS, hiring strategy, and training capabilities are illustrated in Figure 32 below. Larger organisations with well-developed training infrastructures tended to deploy cheaper and less skilled resources (mainly graduates) at entry level to undertake simpler tasks, leaving the more complex tasks to skilled and experienced resources. Because it costs more to recruit people with greater work experience relative to graduates, Organisations A, E, F, and G hired mostly for entry level roles and utilised the capabilities of their training infrastructures to train graduates in a narrow range of competencies, and provided them with opportunities for gradual career development. The extent to which these organisations could hire graduates with lesser skills or from Tiers 2 and 3 educational institutions depended partly on their capability to train people with low knowledge and skills and, partly on their ability to simplify and standardise tasks using their QMS. Job streaming (simple to complex roles) was made possible through the strength of its QMS and associated competency mapping exercise. This demonstrates how QMS support a firm's cost leadership and differentiation strategy in operation. This approach is logical as a significant percentage of development work sourced to India is still at the lower end of the continuum of the software development lifecycle.

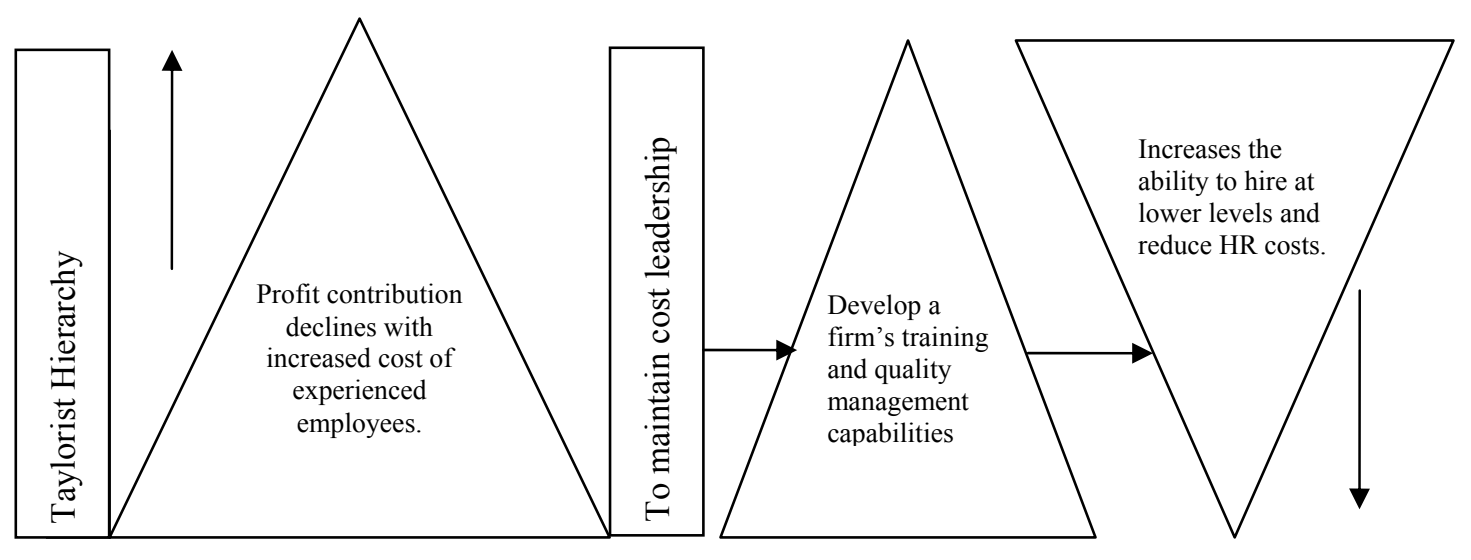

Figure 32. Evolving training capabilities and human resource costs. 
Thus, the focus of large firms' production function is to ensure predictability of service delivery at competitive prices. To ensure predictability, the majority of employees are trained in a narrow range of tasks. These employees are seldom aware of the "bigger picture". Only a small percentage of supervisory and managerial staff had to be trained in people management, interpersonal skills, quality management, and other general skills. Although employees are part of work teams, the amount of discretion they can exercise is limited by the complexity of tasks performed.

Empowerment and autonomy were evident to a limited extent and were mostly exercised by supervisory and managerial staff or by staff in highly complex and new roles. Except for organisations A, E, F, and G, none of the organisations actively promoted job rotation. Thus, the ability the organisations that did so to redeploy resources quickly were much stronger and met the needs of a dynamic services environment.

\section{Employee Turnover}

Employee turnover and training volume were medium to high in most case organisations studied and impacted positively on training volume and diversity. Further, employee turnover levels varied within different service lines in a firm. Employee turnover varied according to the nature of the process and the demand for those particular skills in the external labour market. With the exception of Organisations C and I, all firms that reported high employee turnover also reported a high training volume. Organisations C's relatively simple and stable service portfolios and Organisation I's single product environment did not necessitate high volumes of training. Organisation D's outsourced vendor-distributed model reduced the overall employee turnover and consequently meant low training volume. As a small start-up firm, Organisation $\mathrm{J}$ had negligible employee turnover but medium levels of training because its systems and processes were gradually evolving.

\section{Temporal and Geographical Dimension of Training}

The inability of extant training demand models to explain the variation in training between firms remains. To better understand this phenomenon, the temporal dimension of a process's or projects' lifecycle is needed. Findings from the cross-case analyses 
confirms significant impact the temporal dimension of a process or project has in explaining training variation in the case organisations studied.

If one applies the classic organisational lifecycle " $\mathrm{S}$ " curve to learning and development and follows the neo-human capital assumptions of Bartel and Litcehberg (1987), an organisation's need for learning should decline with experience on a given process or technology. While this theory may hold true for stable and simple process environments, it is falsified in a dynamic outsourcing services environment that is witnessing high growth rates. The following section explains why this is the case.

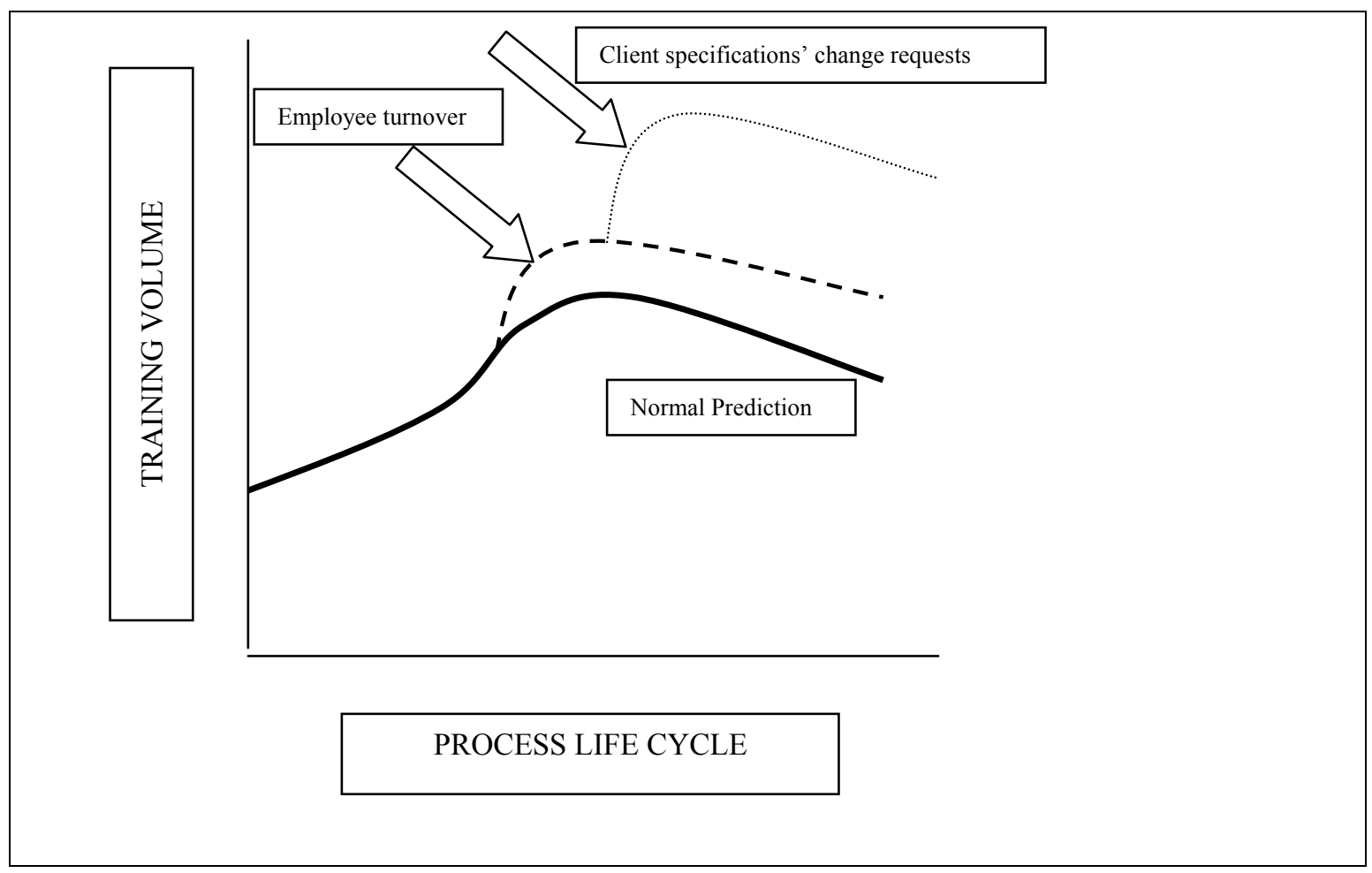

Figure 33. Temporal dimension of process life cycle and training volume.

Although an organisation's training needs should decline as it develops maturity in a given process or technology, additional training is needed because of two key factorshigh and ongoing employee turnover and the constant process, product, or project enhancements requested by the clients. The nature of the changes requested has a stronger impact on training in the ITeS/BPO sector than in ITSS, as the context and the nature of process knowledge is not static as it is in software development, where technology, software applications and the programming logic and language used cut across geographical borders. Figure 33 above captures the dynamism of a temporal dimension of training. 
Overall, in Organisations A, B, and C, high turnover and requests for process changes or enhancements demanded further training. Organisation D's stable processes and an outsourced vendor network model minimise the extent to which it needs to directly invest in training. If the size of the change requested is small, it usually results in refresher training. Major changes to a process, product, or the service level agreement more often results in the development of formal training programmes.

Although the ITeS/BPO and IT software (service) firms start off with a typical "S" curve, additional training "spikes" or new "S" curves are formed owing to ongoing employee turnover and changes to a client's processes. However, a different pattern is observed in software product development firms (Organisations E, F - in one of the software product lines-and I). The product development service lines do not follow a typical "S" curve; their learning and development needs remain constant during the initial stages of product development, as the development team learns by working and in an informal way. Only when the product is launched do the organisation's development and maintenance teams and its clients undergo formal training. Training in ITeS/BPO organisations is also influenced by the geographical location of their clients. Differences in home country legislation, accent, technology applications, and unique cultural factors influence the nature and extent of training when a firm transitions processes from different countries to India. The impact on training remains even if the process is in the same domain (for example, an accounting and finance process for the insurance or telecom industry sectors in the UK and the US).

\section{Firm Performance or Training?}

As noted in the first chapter of this thesis, the focus is to understand the drivers of training rather than how training impacts on organisational performance. The researcher believes that organisational performance is far too complex a phenomenon to be attributed exclusively to training capabilities, as it is influenced by a range of internal and external factors. Although this thesis departs from the extant models that attempt to link training outcomes to firm performance, some inferences on the linkage between the two could be offered in view of the findings that emerge from this study. 
Firms from the US and the UK are known for their short-term focus on return on investment and the need to service one of their key stakeholders- the shareholders (Finegold, 1992). The case analysis revealed issues in developing performance measures in a dynamic outsourcing and offshoring environment. For example, most

Table 18

Comparative Table of Growth in Employee Numbers of Case Organisations

\begin{tabular}{|c|c|c|c|}
\hline Organisation & $\begin{array}{l}\text { Employee numbers } \\
\text { (December2005- } \\
\text { January 2006) }\end{array}$ & $\begin{array}{l}\text { Employee numbers } \\
\text { (December 2008) }\end{array}$ & Researcher's notes \\
\hline A & 26,000 & $37,500+$ & Upbeat on further expansion. \\
\hline B & $\begin{array}{l}1500 \\
(900 \text { in ITeS /BP0) }\end{array}$ & $\begin{array}{l}\text { Not available. New } \\
\text { legal entity. }\end{array}$ & $\begin{array}{l}\text { Leased its ITeS/BP0 business to another Indian ITeS/BP0 } \\
\text { firm in mid-2006 for a period of } 10 \text { years. Retained its IT } \\
\text { networking services business. }\end{array}$ \\
\hline $\mathrm{C}$ & 200 & $450+$ & Growth in ODC business line. \\
\hline D & 70 & $\begin{array}{l}\text { Not available. New } \\
\text { legal entity. }\end{array}$ & $\begin{array}{l}\text { Sold to a small venture capitalist specialising in ITeS/BP0 } \\
\text { following the departure of key senior management. }\end{array}$ \\
\hline $\mathrm{E}$ & 35,000 & $56,000+$ & Wait-and-watch stance for future expansion. \\
\hline $\mathrm{F}$ & 40,000 & $100,000+$. & $\begin{array}{l}\text { Post credit crunch the projected annual growth in employee } \\
\text { numbers for } 2009 \text { is about } 20,000 \text {, of which } 70-80 \% \text { is } \\
\text { expected to be from graduate engineering colleges. }\end{array}$ \\
\hline G & 2,700 & $15,000+$. & $\begin{array}{l}\text { Increased reliance on campus recruitments. Cautious stance } \\
\text { for future expansion. }\end{array}$ \\
\hline $\mathrm{H}$ & 800 & Not available. & Closed in mid-2008 \\
\hline I & 150 & 200 & Modest growth. \\
\hline $\mathrm{J}$ & 36 & $\begin{array}{l}\text { Not available. New } \\
\text { legal entity. }\end{array}$ & $\begin{array}{l}\text { In 2006, the numbers grew to } 75 \text {. In early 2007, it was sold to } \\
\text { a large diversified MNC with SAP specialisation. }\end{array}$ \\
\hline
\end{tabular}

Note: Researcher's notes were compiled using media releases and follow-up discussions with some senior HR and country managers of the operational organisations. 
ODCs (Organisations A, C, E, G, I, and J), while cost centres of MNCs, ultimately contribute to their parent organisation's profitability. They do not employ the conventional performance measures noted in the extant literature. As the case analysis suggests, there is a high employee utilisation rate (85-90\%) mandated by service providers. Thus, any growth in employee numbers can be seen as corresponding to growth in a firm's revenues. A synopsis analysis of the comparison between employee numbers at the time of data collection in the December 2005/January 2006 period and those at the end of 2008 is presented in Table 18.

It appears that firms that had well-developed training infrastructure (A, E, F, and G) and strong QMS also reported growth in employee numbers and business activity. Further, organisations that were developing their training and organisational capabilities $(\mathrm{C}$ and $\mathrm{I})$ were able to maintain modest employee growth rates. The remaining firms $(B, D, H$, and I) who had either relatively weak or evolving organisational capabilities were unable to sustain growth and have been either closed or leased/sold to other firms. One inference that can be drawn from such data is that interaction between the strength of certain organisational capabilities, such as firms' training infrastructure, LO, MO, and QMS capabilities appear to offer superior performance, which may be a source of a firm's sustained competitive advantage.

\section{Conclusion}

This chapter provided a cross-case analysis of firms in India's IT sector. The analysis reveals great diversity in the nature and extent of training in case enterprises yet notable patterns of similarity between them. The problems in measuring the volume of training and firm performance were noted and alternative strategies suggested. The training provided was predominantly technical in nature and the content was fast- changing because of technological and process obsolescence and client requests.

A firm's competitive strategy and QMS have a strong impact on the development of numerous organisational capabilities and consequently shaped the nature and extent of training provision. The linkages between organisations' competitive strategy and HR strategy were noted and in large and well-established firms a close link was found with operations. Despite the hype about "knowledge work" in high technology firms such as 
IT firms, there was strong evidence of digital Taylorism and an application of labour process theory principles using an organisation's quality management capabilities.

Finally, the chapter provided an analysis of various factors that appear to influence provision of training. Technological change, QMS, client specifications, and employee turnover were noted as critical factors in explaining training provision. Geographical location and the temporal dimension of a process life cycle were also examined to explain the differences in training at various stages of a process life cycle and how they impact on training provision. Their impact varied for different sub-sectors (ITeS/BPO and ITSS). 


\section{CHAPTER 7}

\section{DISCUSSION}

\section{Introduction}

Informed by theoretical perspectives used in this study and findings from the within- and cross-case analysis, this chapter answers the study's main research questions and presents a theoretical framework for understanding of the factors that influence provision of training in India's IT sector. This chapter concludes by offering an explanation for variation in training between firms.

\section{Research Questions and Developing a Theoretical Framework}

As expressed in chapter 3, the study identified four research questions. A summary of key factors identified in the preceding chapters answers the first research question:

\section{What are the main factors that influence a firm's decision to invest in training in IT sector, within the Indian context?}

Contrary to the earlier conceptualisations that considered that training is driven primarily by factors that are internal to the firm (Ashton \& Sung, 2006; Smith \& Hayton, 1999; Ridoutt et al., 2002), this study found that it is a combination of, and complex interaction between, various internal and external drivers that influence training decision making in the organisations studied. Internal factors that influence a firm's training decision making are as follows:

- competitive strategy

- workplace change and organisation of the production function

- organisational capabilities

- market orientation

- learning orientation

- quality management systems

— HRM practices

- the temporal dimension of a process

- employee turnover 
- enterprise size, age and ownership.

External factors that influence a firm's training decision making are as follows:

- client specifications

- process/project complexity

- the geographical dimension

- gaps in the educational curriculum in skills needed by the IT industry

- external labour market dynamics.

Strong support was found for the second research question:

\section{Do an organisation's clients/customers have an impact on investment in training? If so, why and how do they influence it?}

Relative to ITSS organisations-product (Organisations F, G, and I) and services (Organisations E, F, H, and J), significant evidence was noted in ITeS/BPO organisations (Organisations A, B, C, and D) of the influence of clients' specifications on the nature and extent of training. The nature of the business model (mostly third-party services) and the diverse nature of business processes had a strong influence on ITeS/BPO firms' training demands. Differences in national cultures, technologies in use, legislation, software applications, and the standard operating procedures and business processes of firms were common drivers for providing client-specific training.

In ITSS projects, the impact of client specifications on training was not as strong as it was in ITeS/BPO firms, as software applications and programming languages are generic and transferable across borders. Nevertheless, depending on the nature of the project, organisations reported a need to provide client-driven training to project members in using certain proprietary tools and applications that were specific to a client organisation or a technology not commonly used by the service provider. Thus, for solution design and architecture teams of ITSS firms, further training was necessary to understand such tools and clients' business logic and processes before software solutions could be designed.

The above is a simple account of a much more complex level of negotiation and exchange between the client and the service provider before final client specifications (through a service level agreement) are mutually agreed between the two. Such 
negotiation occurs through dynamic coordination (Banerjee, 2004) and is linked to the varying levels of an organisation's capabilities. Organisations exercise choices to invest in developing certain capabilities. These capabilities evolve over time and through dynamic coordination between the service provider and the client organisation. In addition to the interactions between various internal and external factors, the aspect of dynamic coordination and strategic demand management (Banerjee, 2004) is explored in detail in the following section to answer the study's third research question.

\section{How do various factors-internal and external to the firm-interact with one another in the provision of training in India's IT sector?}

The interaction between various internal and external factors takes complex forms and is to some extent explained by the different strategic milieus that organisations operate in. Figure 34 presents a theoretical framework of the key factors that influence the provision of training in India's IT sector. The theoretical framework for enterprise training developed in this study is grounded in the confluence of various contributing, useful, and novel theories (Swanson, 2007). Most of the above factors can be linked back to different disciplines and theoretical orientations (for example, economics (neo-HCT, competitive and business strategy), marketing and management (market-based organisational learning and quality management), and human resource management (studies of HPWPs, SHRM practices, and resource-based view of the firm)). The framework presented provides answers to the third and fourth research questions. For ease of understanding, the framework is explained as follows:

- the key elements and interaction between internal and external factors

- the variation in training between firms.

\section{Key Elements and Interaction between Internal and External Factors}

The two dotted lines in Figure 34 represent the porous nature of the boundaries of the information and of the resource exchanges that occur between the IT sector of India, its local labour market, and the global off-shoring and outsourcing client firms. It is in this dynamic coordination and global context that enterprise training needs to be understood. 
Figure 34. A theoretical framework of influences on enterprise training

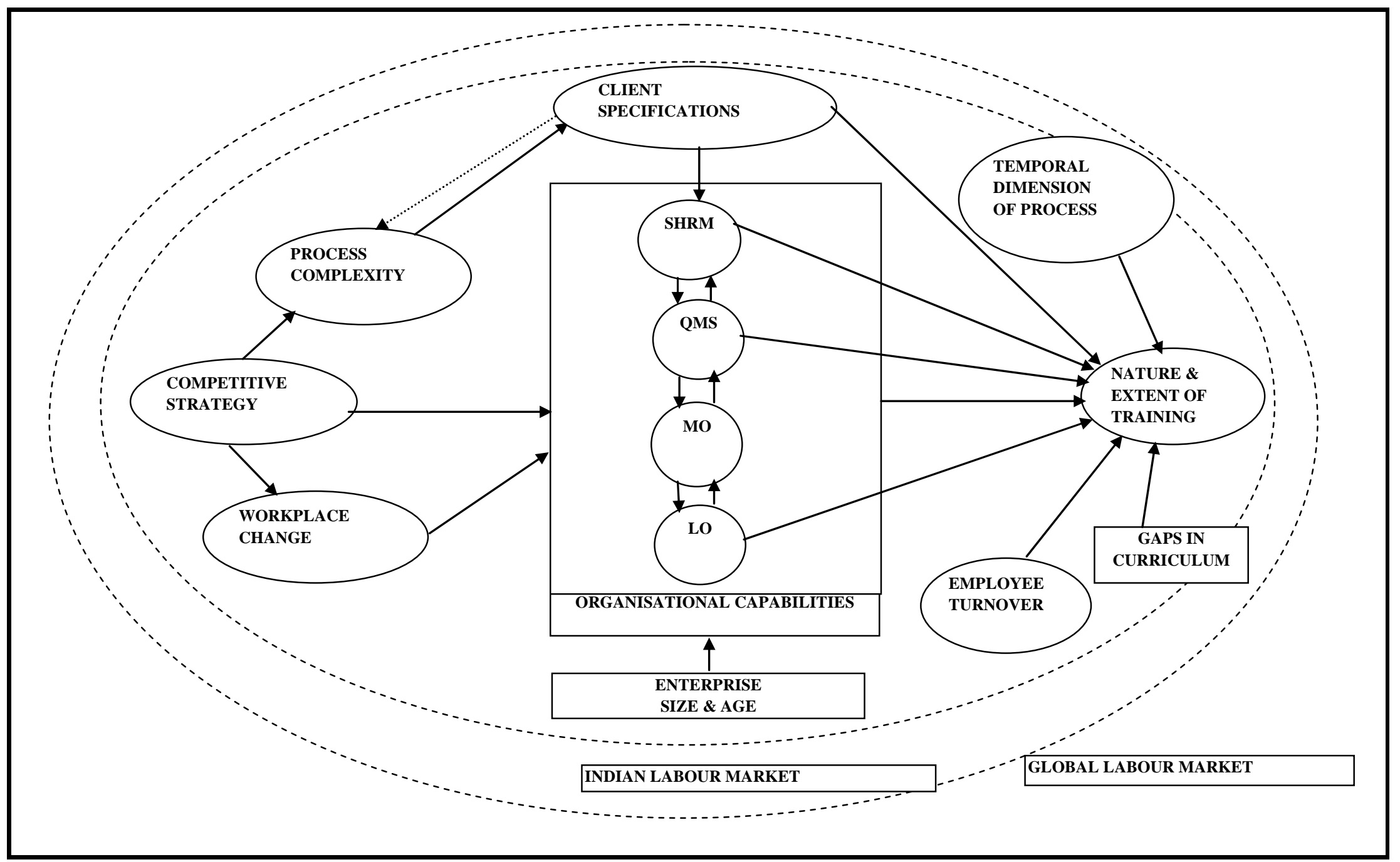




\section{Competitive Strategy}

Although a firm's competitive strategy sets the tone for the markets a firm operates in and may represent one or a combination of Porter's (1985) three generic strategies, there was always an element of emergent strategy noted, which co-evolves as a result of the dynamic coordination between the client and service provider firms (Banerjee, 2004, 2006). It is evident from the case analysis that firms elected at the outset to compete in either mass service, slightly differentiated, or highly differentiated markets or a combination of them (Boxall, 2003). Later, as the opportunities emerge as a result of the dynamic coordination between the client and the service providers' capabilities, the strategies to service in different markets co-evolve (Banerjee, 2004, 2006).

The above service differentiation sets the tone for an organisation's business strategies such as its people management and skills strategy, which has a profound impact on training decision making. As noted in the previous chapter, firms in mass service or in slightly differentiated service markets invested relatively less in training volume than firms operating in highly differentiated service markets. The choice of a firm's competitive strategy and service markets informed the nature of the projects or processes it sought to service. Depending on the service markets in which a firm operates, process or project complexity varies. Relative to the slightly and highly differentiated services, process complexity is generally low in mass service markets. Consequently, clients' specifications vary in complexity.

\section{Workplace Change and Work Organisation}

As noted in chapter 6, technological changes and investment in the development of certain organisational capabilities necessitate the development of new skills. Technical, rather than structural, workplace change seems to impact more on training diversity and volume. Technological changes have an ongoing impact on training provision for ITSS firms owing to the high rate of technological obsolescence characteristic of the sector.

There was little evidence of workforce restructuring to reduce employee numbers. On the contrary, the focus was on expanding operations in industry-specific specialisations to gain economies of scale and develop in-depth domain expertise. Changes from one 
business model to another (from an outsourcing to an offshore development centre model or to a combination of both) also had a profound impact on training provision, as was amply demonstrated in Organisations A, B, and C.

The more an organisation developed its quality management and training infrastructure, the more easily it was able to implement modified Taylorist forms of work organisation and hire recent college graduates. This ability was particularly true for large ITSS firms; for example, Organisations E, F, and G had well-developed QMS and large training infrastructures. To service their competitive strategies, investment in QMS and a modified Fordist or Taylorist work organisation was appropriate.

In ITeS/BPO firms, the nature of most services being studied was simple, so a lot of work organisation followed Taylorist principles. Under this approach, the level of employee discretion is generally low and employees work on well-defined tasks and are provided with focused training to complete their tasks. Focus on career development is absent or limited. Service lines that apparently adopted a more modern, team-based approach were typically firms or business lines representing new and highly complex services.

The business processes for these services were gradually maturing, and the nature of learning for these processes relied more on informal and incidental forms (Watkins \& Marsick, 1992). Further, the ability of the organisations to develop internal service standards for new services was limited, at least initially. The above process teams started with an informal, team-based structure (which gives the impression that employees have more discretion and say over how work needs to be completed and what learning needs to be provided). Nevertheless, with time and experience, and keeping in mind an organisation's capability to standardise operations, the training needs of these teams are expected to decline, although they do not always do so. This theoretical anomaly is explored further in the next paragraph.

\section{Temporal Dimension and Employee Turnover}

In a dynamic outsourcing environment, the temporal dimension of a process's life cycle does not follow the neo-human capital assumptions (Bartel \& Litechenberg, 1987) of a decreasing need to train with increases in experience of a technology/process. Instead, 
training needs are constantly driven by changes in clients' process specifications and high levels of employee turnover. As the sector is witnessing high growth rates and an increasing in the number of new firms are setting up their shops in India, there is a tendency amongst new firms to poach trained resources from established firms. Thus, increased competition in factor markets and high employee turnover in established firms have a direct and ongoing impact on the nature and extent of training. The extent to which changes to client specifications are implemented in a process is influenced by the complex interaction of a firm's capabilities. An understanding of the interactions between organisations' LO, MO, QMS and HRM systems is necessary to demonstrate how the nature and extent of training is finally shaped.

\section{Organisational Capabilities}

Organisations possess numerous capabilities that serve different purposes. For the purposes of the study and based on the data collection and analysis, this thesis focuses broadly on two sets of capabilities: people and client management capabilities. These capabilities exist on a continuum, from highly developed to minimally developed. In particular, the interactions among an organisation's quality management systems (QMS), its learning orientation (LO), market orientation (MO), and the human resource management (HRM) infrastructure are discussed here. It is neither desirable nor practicable to discuss here these four organisational capabilities in isolation; instead, an integrative approach is considered pragmatic. Such an approach allows for a holistic understanding of the systematic interactions between the various organisation capabilities.

The analysis found that the critical and unexplored link between an organisation's MO and LO abilities is the enabling role that its QMS play in sensing, disseminating and framing organisational responses. It is important to note that the position of an organisation's QMS (on a continuum from weak to strong) affects the development of its $\mathrm{MO}$ and LO abilities.

When a new process or project is received by an organisation, its ability to sense and disseminate the process/project information is affected by the strength of its MO, LO and quality management capabilities. Although LO and MO have been linked in earlier frameworks (Sinkula et al., 1997), there is no evidence of how these capabilities are 
developed in a dynamic services environment. Within- and cross-case analyses suggest that an organisation's QMS appear to be mediating the development of an organisation's MO and LO capabilities. Depending on where these capabilities are on a continuum of being highly developed to being minimally developed, the operational and training responses will differ.

For example, if an organisation has high MO and medium to low LO and QMS, it is likely to demonstrate adaptive learning, as the extent to which it can engage in critically challenging its clients' assumptions depends on the extent to which its internal benchmarks of performance are developed. To be able to challenge clients' assumptions (typical of strong LO), it is necessary first to have well-developed internal process benchmarks, performance standards, or prior experience of a process as the basis on which to challenge the clients' assumptions. The development of such standards is enabled by the presence of a strong QMS or internal performance standards developed over a period of time. For example, Organisations B and C did not have strong QMS or performance standards and were therefore unable to develop the client metrics necessary for engaging in higher order learning and framing high quality responses. This does not mean that the organisations produced low quality work, but rather that the quality of their service deliverables reflected the maturity of their QMS capabilities and was what they were able to negotiate with their clients in their SLAs.

However, as Boxall (2003) argues, the opportunity to invest in quality is high in firms competing in highly differentiated service markets, where there is an opportunity to realise economic gains found partial support. The implementation and development of organisational capabilities should have a business impact to be sustainable in the long run. For example, if a client does not want high level of investment in the quality of its business processes, then all the service provider can do is to improve the productivity of their internal processes and realise cost savings. Similarly, processes that are close to mass service or slightly differentiated markets do not provide full economic rationale for sustained investment in QMS or a bundle of HPWPs. Overinvestment in QMS can be counterproductive.

Boxall (2003) further argues that certain capabilities that are unique in sustaining competitive advantage over other firms may be emulated by other firms in the sector and may then become a given or a "table stake". It follows from the case analysis that 
the increasing proliferation of quality management systems is now becoming a "table stake" in a dynamic outsourcing environment for enhanced market signalling, cost reduction, and service excellence. However, mere adoption of a particular quality management standard or system does not guarantee sustained high performance or differentiation. QMS, like other capabilities exist on a continuum. The extent to which it is developed and the unique organisational configurations in which it is applied, is what matters and what creates differentiation. Resource theorists (Barney, 1991; Leonard, 1998; Wernerfelt, 1984; Wright et al., 1994) argue that the ability to deliver sustained high performance is the function of the organisation's ability to recombine its capabilities with other resources, which also depends on the organisation's path dependence, leadership commitment, and social complexity.

Where an organisation's QMS are well developed, stronger LO and MO abilities can be developed, as was noted strongly in Organisations A, E, F, and G. Strong QMS, which focus on predictable service delivery in this context, paradoxically also help in developing generative and transformational learning (Argyris \& Schon, 1978; Senge, 1990). Here, it appears that QMS have strong links with an organisation's ability to standardise and innovate and, with its learning culture and temporal dimension of processes or projects.

The presence of a metrics-driven language evident in QMS set the tone for MO and LO. For example, the quality management systems of Organisation A were extremely well developed and had a flow-on effect on to MO and LO at various stages of the processes' acquisition, transfer, delivery, and performance. From a training perspective, for example, Organisation A's ability to precisely identify the training needs was much stronger than the ability of Organisations C, D, I, and J. The latter organisations were still in the process of adopting and developing their QMS and these had limited ability to define metrics to influence and improve service delivery.

QMS directly influence LO and MO because they also involve collection and dissemination of information based on pre-established standards of performance. QMS focus both on the hard aspects (cost, continuous improvement, top management commitment) and soft aspects (team working, training, information sharing) aspects of performance. In other words, QMS enable the development of standards of performance using a range of tools and techniques from its stable. The ability to granulise the 
information and reflect on it develops with experience of numerous quality methodologies and business processes. An organisation can find out what is do-able and where process improvements can be made through use of these quality tools and experience of QMS. The ability of Organisations A, E, F, and G to make use of such quality tools in framing their solutions has increased with experience. The ability of smaller organisations like C, D, I, and $\mathrm{J}$ is likely to improve over time and with continued investment in building its QMS.

QMS also influence the degree of control management can exercise over work processes and employees' activities. The findings suggest that for processes that are more complex in nature or more differentiated (Boxall, 2003), an organisation can exercise control only to the extent to which it can simplify these processes. This finding is also critical for an organisation's recruitment and selection, performance management, and skills strategy.

If an organisation can simplify its processes into narrow tasks and activities, it can continue to deliver predictable levels of service at lower cost. It can do so by hiring people at entry level with limited experience, certain base qualifications, and by training them in a narrow range of competency areas, as was borne out by most large organisations (A, E, F, and G). All of these organisations had extremely well-developed QMS, strong LO and MO, and a focused HR and skills development strategy, with training infrastructure to support it.

In addition to the enabling role QMS has on the development of certain capabilities, the findings of this thesis depart from the extant literature in relation to the way training and development is structured in an organisation. Traditionally, training has been seen as part of the bundle of a firm's HRM activities; however, in most organisations studied (A, B, C, D, F, G, H, and I), training was structured as an integral and inextricable part of operations. Organisation E used its Global Campus corporate university content and relied strongly on external training providers. Although it relied on a mix of internal (mostly through informal learning) and few external (formal training) providers, Organisation $\mathrm{J}$ had decided to set up a training infrastructure in the short term. Strategic HRM practices were focused more on recruitment of a specific group of employees and retaining them by managing their career development needs. 


\section{Gaps in the Educational Curriculum}

Firms in the Indian IT sector have to constantly confront the gap between what the industry needs and what the educational curriculum of engineering and non-engineering graduates produces (NTITSD, 2003). Only $20-25 \%$ of the overall graduates are directly employable with minimal training input. In most cases, their ability is limited to undertaking relatively simple tasks. Thus, providing process- and domain-specific training and some project experience is inevitable.

\section{Enterprise Age and Size}

What also follows from the analysis is the organisation's ability to invest in certain critical capabilities that consequently shape training has an element of path dependence and social complexity in it (Barney, 1991; Leonard, 1998; Wernerfelt, 1984). In line with the resource-based view of the firm, large organisations (A, E, F, and G) amply demonstrated the significance of enterprise age and size, more specifically, its process complexity and capabilities, and how they influence skills development. The following section attempts to answer the final research question of this thesis.

\section{Why do some firms invest more than others even if they are in the same industry sector?}

\section{Variation in Training between Firms}

In answering the study's last question, it is important to understand the reasons why firms in the Indian IT sector invest in training. Once the reasons are understood, explanations for the variation in training between firms can be advanced. From the study's analysis, the main reasons for investing in training by firms in India's IT sector are as follows:

\section{Employee performance}

All case organisations needed to equip employees with the necessary skills to perform tasks in new processes and technologies. Training was often provided in narrow role competency areas by large organisations (A, E, F, and G) to reap economies of scale 
from their training infrastructure. Even smaller organisations $(C, D, I$, and $J)$ provided role- and process-specific training.

\section{Costs and strategy}

Large organisations (A, E, F, and G) considered training as a strategic vehicle for reducing the cost of delivering services. These organisations invested heavily in their training infrastructures and had a strong graduate recruitment programme. The nature and extent of the training and the extent to which they invested in other capabilities, reflected their competitive and business strategies.

\section{Clients' specifications}

In a dynamic outsourcing environment, ITeS/BPO firms (A, B, C, and D) and, to a lesser extent, ITSS product (E, G, and I) and service firms (F, H, and J) considered training on client specifications critical in satisfying clients' latent and expressed needs. Such needs were met by the varying levels of an organisation's people and client management capabilities.

\section{People and client management capabilities}

Training supported the development of strong QMS and LO, MO and HRM capabilities. Large organisations (A, E, F, and G) were able to demonstrate high growth rates and deliver high quality services and solutions. Those in the process of developing the above capabilities (Organisations B, C, D and J) were also able to post growth rates.

\section{Employee retention and career aspirations}

Larger organisations growing at a higher-than-average rate (A, E, F and G) needed to retain and add more employees on an annual basis. Paradoxically, employee retention increased as firms improved the marketability of their employees. Paying attention to career aspirations meant providing employees with opportunities to develop new sets of technical, management and leadership skills. Further, given the high rate of skills obsolescence in technological areas (as noted in Organisations I and J), employees do 
not want themselves to be seen as "stuck in the middle" or "working on an old technology".

\section{Temporal Dimension of Process and Projects}

Variation in training is better explained by the complex interactions between an organisation's competitive strategy, external and internal labour market dynamics, the temporal dimension of processes, and the organisation's evolving set of people and client management capabilities. For example, an organisation may be operating in a range of service lines that typify slightly and highly differentiated service markets, thus requiring different levels of training provision. Further, according to the extent to which an organisation has accumulated experience in a service line and to which process changes are requested, various levels of training will be necessary. If the rate at which a particular service line is experiencing growth is high, the demand for skills in that service area will increase in the external labour market. Consequently, high employee attrition rates limit retention and affect a firm's ability to hire effectively from a finite pool of campus graduates. The effect is higher levels of training provision in certain industry specialisations, either internally or externally.

\section{Conclusion}

This chapter provided answers to this study's research questions. In answering the study's questions, a conceptual framework for understanding the factors that influence provision of training was presented. The decision to invest internally or externally is linked to the HR and skills strategy and is influenced by the organisation's people and client management capabilities. There is no straightforward explanation for variation in training between firms; it is dependent on the firms' specific internal and external strategic milieus. Thus, according to the extent to which an organisation's LO, MO, QMS and HRM capabilities are developed; varying effects on training volume and diversity are expected. The chapter concluded with reasons for the differences in training provision between firms operating in similar strategic milieus. 


\section{CHAPTER 8}

\section{CONCLUSION}

\section{Introduction}

This final chapter builds on the findings and analysis from the previous two chapters. First, it discusses the implications for policy-makers, practitioners, and theory-building. Second, limitations of the study are highlighted. Finally, this chapter concludes with propositions for future research. These propositions can form the basis for further case study-based or survey research.

\section{Implications for Policy and Practice}

The findings from this study have direct implications for practitioners and policy makers. Despite the number of national taskforce analyses focusing on the human resource development needs of the IT sector of India (NTITSD, 1998, 2003; NASSCOM-KPMG, 2004), the findings still indicate that the curriculum is not responsive to market needs. A major change in mindset by educational providers and policy makers is needed. While curriculum content is critical, equally, it is how the curriculum is taught and the speed with which it can respond to market needs are considerations for policy makers. Industry- ready talent requires a mix of general, technical, domain and industry-specific skills. Although recent initiatives by the industry's professional and policy development body, NASSCOM, are steps in the right direction, their focus on application and moving up the value chain is rather limited.

Larger firms with well-developed training infrastructures may not be as affected as the new start-ups or relatively small and medium-sized firms. The latter group relies on skills acquisition from an already competitive labour market. Consequently, the wages increase and the comparative labour-cost advantage that India had is gradually eroding. The following section discusses NASSCOM's key IT sector initiatives (NASSCOM, 2009) and how well they address the policy implications. 


\section{Capacity Building}

First, the Ministry of Human Resource Development has included in the 11th five-year plan the addition of 20 more Indian Institutes of Information Technology (IIIT). Secondly, NASSCOM and the Government of India plan to issue targeted $\mathrm{PhD}$ fellowships in computer science and information technology. Further, to bridge the gaps in curriculum, faculty development programmes have been launched at a national and state level. While the problem of supply may be resolved in the medium to long term, the issue of application of concepts, and knowledge and application of emerging technology still exists. Although the students may be conceptually strong, most have never worked in a real software development project environment before graduating. This is an area where industry-academia partnerships will be valuable.

\section{National Assessment Centres}

To create an industry-ready talent pool, NASSCOM and large IT companies have identified generic competency areas necessary for employment in ITSS and ITeS/BPO sectors. Assessment centres in 13 states in the country have been established and close to 21,000 students have undertaken assessment so far. The objectives of this exercise are to create a national repository of skilled graduates, reduce screening costs and time, and identify competency gaps. While this may be a smart move by the industry and useful for small and large players in reducing their recruitment and possible training costs, the downside of this initiative is the transferring of costs for assessment and any resultant training to the individual. Consequently, it may reduce the uptake of these assessments.

\section{Capability Development}

Other NASSCOM initiatives focus on providing continued professional development courses for specific sectors and developing finishing schools for honing in on IT and soft skills, using self-paced and open and distance learning delivery modes. While these initiatives will certainly help in overall capability development, the issue of costs transfer and responsibility might become a deterrent to the uptake of such schemes. Development of additional knowledge and skills should be embedded in the teaching content and its delivery. 
In addition to the above policy issues, much of the focus in both ITSS and ITeS/BPO firms is to cater to the low-end services continuum. The large Indian IT firms have yet to make an impact by developing world class software products. High upfront costs and limited expertise in research and development are significant deterrents. Given the credibility the sector currently enjoys, further investment in new areas of research and development would be timely to take the growth and skills development trajectory to the next level. Global collaborative ventures between industry, government, and academia are needed to enhance the sector's technological prowess.

Organisations and practitioners can benefit from understanding the critical factors that enable the development of strong learning cultures and organisational capabilities necessary for higher order learning. Further, an appreciation of the relationships between different levels of an organisation's capabilities is necessary to understand differences in firm performance between small and medium to large firms. Practitioners need to be aware that in dynamic outsourcing environments, almost $95 \%$ of employees are billable resources. Employing more than 5\% staff in enabling functions is considered as a sign of complacency. Further, having about $5-10 \%$ of employees on "bench time" for switching ease (from product or project environment), is a luxury only larger firms can afford due to their efficient production function and organisational capabilities.

Although certain quality management accreditations and tools may offer cost benefits, however, the extent to which an organisation recombines its resources to produce higher order competencies, different levels of benefits will be achieved. Practitioners also need to be aware of training dynamics in firms with short product and process lifecycles. The implication is that firms will need to invest more in skills and capability building when they have shorter product and process lifecycles, especially if process/project diversity and dynamism is high.

Despite the significant amount spent on training, training evaluation wasn't well developed. Most training evaluation was comparable to Kirkpatrick's (1998) first two levels. Evaluation had a narrow focus and relied on tools from quality management frameworks. The findings also indicate that employee turnover does not always have negative effects. Knowledge spillovers and the resultant growth of the IT industry have been beneficial (Banerjee, 2004) and in some cases support the cost leadership strategy. 
Moreover, in a dynamic and high growth environment, a preoccupation with containing employee turnover may be futile. Nevertheless, depending on an organisation's strategic milieu, better job design, motivating employees, and providing them with opportunities to improve their employability may be better retention strategies.

\section{Implications for Theory Building}

This thesis examined a number of theories that explain why firms invest in training. Findings were analysed using the study's contributing, useful and novel theories (see Chapter 3 for details). Implications for theory development are presented in this section. In general, theories from economics, management, marketing, and operations management literature were examined. The following section considers the extent to which the study's findings can be generalised to the above theoretical perspectives (Yin, 2003; Eisenhardt, 1989). For this purpose, it would be timely to reproduce the main propositions of the study, its theoretical orientations, and the extent to which the study's findings offer full, partial, or no support (see Table 19). Next, implications for theory follow.

Table 19.

Key propositions and study's findings

\begin{tabular}{|c|c|c|}
\hline Propositions & $\begin{array}{l}\text { Theoretical } \\
\text { orientations }\end{array}$ & Findings \\
\hline $\begin{array}{l}\text { Proposition 1: Firms will invest more in firm-specific training than } \\
\text { general skills training. }\end{array}$ & HCT & Not supported \\
\hline $\begin{array}{l}\text { Proposition 2: Firms will invest in general skills training due to } \\
\text { asymmetrical market information about their new employees. }\end{array}$ & Neo-HCT & Not supported \\
\hline $\begin{array}{l}\text { Proposition 3: The demand for highly skilled workers will decline } \\
\text { with experience on a given technology. }\end{array}$ & Neo-HCT & Not supported \\
\hline $\begin{array}{l}\text { Proposition 4: Where the focus of implementing new management } \\
\text { practices is on increasing employee autonomy and discretion, firms } \\
\text { are more likely to invest in general and technical training. Where the } \\
\text { focus of such practices is on high employee control and low } \\
\text { employee discretion, firms will invest in technical training. }\end{array}$ & $\begin{array}{l}\text { HPWPs and } \\
\text { SHRM }\end{array}$ & Partial support \\
\hline $\begin{array}{l}\text { Proposition 5: Firms focusing on a cost leadership strategy will } \\
\text { invest less in training and quality management systems. }\end{array}$ & $\begin{array}{l}\text { Competitive } \\
\text { strategy \& } \\
\text { TQM }\end{array}$ & Partial support \\
\hline $\begin{array}{l}\text { Proposition 6: Firms servicing slightly and highly differentiated } \\
\text { service markets will invest more in training and quality management } \\
\text { systems than firms servicing mass service markets. }\end{array}$ & $\begin{array}{l}\text { Competitive } \\
\text { strategy, } \\
\text { SHRM \& } \\
\text { TQM }\end{array}$ & $\begin{array}{l}\text { Partial support. } \\
\text { Mixed results. }\end{array}$ \\
\hline $\begin{array}{l}\text { Proposition 7: The strength of an organisation's quality } \\
\text { management systems helps in shaping the nature and extent of } \\
\text { training provision. }\end{array}$ & TQM & $\begin{array}{l}\text { Strongly } \\
\text { supported }\end{array}$ \\
\hline $\begin{array}{l}\text { Proposition 8: In high-tech services environment, high levels of } \\
\text { market and learning orientation will require higher levels of } \\
\text { investment in technical and general skills training. }\end{array}$ & $\begin{array}{l}\text { Strategic } \\
\text { marketing and } \\
\text { management }\end{array}$ & Partial support \\
\hline
\end{tabular}




\section{HCT}

Under HCT, the distinction between generic and firm-specific skills development suggests that firms will invest more in firm-specific skills development than in generic and transferable skills development. The case findings concur with earlier issues regarding the blurred distinctions between generic and firm-specific skills (Maglen, 1990; Strober, 1990). Further, in an outsourcing environment, the above distinction is not relevant because of the ongoing need to invest in skills that are not only new, but also not readily available from the external labour market. These skills include understanding clients' unique standard operating procedures, business process specifications, new technologies, and legislative changes in different cultural contexts (see, for example, the influence of client specifications and temporal dimensions of a process/project in Figure 34 on the nature and extent of training). Further, to accommodate employees' aspirations and maintain market leadership, investment is made in emerging technologies and new applications training, even if there is no immediate benefit realised. Thus, the first proposition is not supported.

Although measuring the impact of training and education on firm performance and productivity is not the focus of this study, inferences can be made from case analysis about importance of several organisational and external influences on productivity, which are outside of the purview of orthodox HCT. This raises concerns for policy makers who are pursuing the "high-skill and high wage route" to national competitiveness and prosperity. The above policy approach assumes that developing countries will be unable to compete for highly skilled jobs and thus, high-wages. However, the above view ignores the impact of an "over supply" of highly skilled resources on "high wages". There is sufficient evidence from China and India that challenges such assumptions.

\section{Neo-HCT}

Acemoglu and Pischke's (1998a, b) neo-human capital assumptions of asymmetrical market information are not relevant here. First, there is well-developed information regarding what the engineering curriculum delivers and what the IT sector requires (National Taskforce on Information Technology and Development, 2003; Narayanan \& Neethi, 2005). Second, most firms have numerous pre-employment tests to assess the 
suitability of prospective applicants. Moreover, the strength of an organisation's capabilities and path dependence (see Figure 34) impact the extent to which firms have asymmetrical market information about potential applicants. Overall, the information asymmetry was found to be low. Organisations (especially large ones) are well aware of the gaps and do not have much choice but to invest in industry-specific human capital and other transferable general training. Firms need to invest in general skills to keep up with changing business models and clients' needs. Thus, the second proposition is not supported.

Bartel and Litchenberg's (1987) thesis that relative to less educated workers, the comparative advantage of highly educated workers declines over time with experience in a technology, is partially supported. It follows that such a prediction may be possible in stable environments, but not in high-growth dynamic outsourcing environments, where employee turnover rates are high and the clients request frequent changes to a process/product in its life cycle, thus necessitating sustained investments in training. This finding is a significant departure from the neo-human capital assumptions above. Even though the overall pool of skilled and qualified workers has doubled globally (Brown et al., 2008), its impact in sectors such as IT in developing countries like India still necessitates sustained investments in training and education. Thus, the third proposition cannot be upheld in a dynamic outsourcing environment. Dynamism in process/project lifecycle and its impact on training is illustrated in Figures 33 and 34.

In line with earlier findings (Cappelli, 1993; OECD/CERI, 1986, 1988), ongoing workplace and technological changes needed investment in training. Proponents of neoHCT (Wozniak, 1984, 1987) found high human capital to be necessary in adopting workplace innovations and new management practices. Smith et al. (2004) found that workplace change associated with the adoption of new management practices that focused on empowering employees and allowing them discretion often resulted in the provision of both behavioural and technical training. In contrast, workplace change and new management practices or HPWPs that focused on employee and work process monitoring and control often resulted in the development of mere technical skills (Smith et al., 2004). Case analysis suggests that there is no simple relationship between workplace change, the focus of new HPWPs, and provision of technical or behavioural training. 
Overall, firms invested more in technical training and less in developing behavioural skills. The findings show partial support for the fourth proposition, because neither high employee control and monitoring, nor high employee empowerment and discretion have a relationship with the provision of technical and behavioural training. There are three main dimensions: career aspirations, role-based skills development, and service complexity. Only where there is scope for, and a need to assume, higher line management responsibilities and client-facing roles do firms provide behavioural training. Behavioural training was also noted in firms operating in a highly differentiated market and whose operations were expanding. This trend is consistent with the opportunity to apply behavioural skills. An understanding of the wider contextual environment in which a firm's production function is organised, helps us make sense of training decision-making.

\section{HRM}

Having training in an organisation is often considered a critical practice in the bundle of universalistic HRM practices (Beer et al., 1984; Kochan et al., 1986; Pfeffer et al, 1998). Further, training and investment in skills for career aspirations is considered a win-win strategy for gaining employee commitment and achieving positive HR and organisational outcomes. Case analysis does not fully support this view. Training and development practices in case organisations were not in an integrated bundle of HR practices. Training delivery was strongly intertwined with firms' operations management in most cases. The above finding has implications for HRM models and theories where, integration of training in HRM practices is considered to be the litmus test for a strategic approach to HRM. Despite the apparent challenges for HRM theory noted above, the findings present opportunities for HR practitioners to engage in evidence-based practice by employing quality management tools. By developing distinctive strategic contributions that a firm's training capability can offer, HRM can regain its prominence and place in executive decision-making.

In some smaller organisations $(\mathrm{C}, \mathrm{D}$, and $\mathrm{J})$, despite their non-existent or minimalist $\mathrm{HR}$ infrastructure, medium levels of training volume and moderate levels of training design and delivery practices were evident. In larger organisations (A, E, F, and G), the role of $\mathrm{HR}$ in relation to training was present, but it generally was confined to the management of its logistical arrangements and the administration of training evaluations. 
Studies of best fit HRM models (Boxall, 2003; Fombrun et al., 1984; Schuler \& Jackson, 1987) show a reliance on contingency factors and the adoption of certain competitive strategies. Although support is found for the fifth and sixth propositions in relation to the nature of competitive strategy, an organisation's service markets (Boxall, 2003) and its impact on training, the relationship is not straightforward.

Firms studied confirm the coexistence of cost leadership and differentiation strategies. The distinction in the literature regarding adopting an either/or (cost leadership or differentiation) competitive strategy to avoid being "stuck in the middle" is questionable (Boxall \& Purcell 2008; Porter, 1985) because most case organisations were competing on price and quality of services. Findings from this study are akin to the economics of high quality production in Japan's automobile manufacturing sector, employing for example - Toyota Production Systems - or applying quality management practices and new automated technologies for improving product (service) quality as well as reducing the cost of product (service) delivery. Further, contrary to extant literature (Boxall \& Purcell, 2008), competition in highly differentiated services do not always require high level of employee discretion and the basis of competitive position is not just based on quality; it also focuses on price. Rather, the competition now requires firms offering highly differentiated services to provide the best overall value proposition (which includes cost and quality).

Further, the dynamic coordination between service provider, client needs, and the external market dynamics constantly reshape the development of these strategies (Banerjee, 2004). This co-evolving nature of competitive strategy influences the level of process/service complexity and the nature organisational changes necessary for supporting the firm's new strategic direction (See Figure 34). Training provision relied on the strengths of a firm's people and client management competencies and capabilities, a finding that is consistent with the resource-based view of the firm (Barney, 1991; Wright et al., 1994). Proposition seven, regarding the influence of a firm's quality management system (QMS) on the nature and extent of training, found strong support. The study also found that a firm's QMS impacts on the development of people and client management capabilities. 
A significant contribution made by this thesis is to link the critical and unexplored roles of an organisation's QMS in Sinkula et al's (1997) market-based organisational learning framework. Partial support was found for the last proposition. Instead of LO on its own, case findings suggest that it is the strength of an organisation's QMS that develops an organisation's LO, MO, interpretation and, thus, its business and training responses (actions). Further, like LO and MO, an organisation's QMS exist on a continuum. Depending on the extent to which the QMS are developed, an organisation's MO and LO can be improved. In a dynamic and high growth outsourcing environment, an organisation's QMS (for example, Six Sigma, Lean Six Sigma, or Malcolm Baldridge frameworks) direct the collection and dissemination of highly granulised information that is critical to service delivery from an internal and external (clients') perspective. Such information and customer orientation provide a focus for shaping the nature and extent of learning that needs to be sensed and disseminated widely in the organisation.

Although Day (1994) has long asserted the need to include techniques from the umbrella of TQM for developing market-based capabilities and decision-making, there is a general paucity of research that examines the impact of quality management orientation on learning and market orientation for improving marketing outcomes. Only recently, research from a developing country context has explored the impact of quality management systems on the development of market-based and organisational capabilities (Malik, 2009). Case findings suggest that not only do an organisation's QMS help in developing its LO and MO, they also shape an organisation's ability to standardise its labour processes and establish strong internal benchmarks for performance. These benchmarks and standards provide the basis for performance management, training needs analysis, and continuous improvement projects, thereby indirectly influencing the development of an organisation's HRM practices. Thus, an understanding of the above capabilities and of their interaction with other factors, and the resource-based view of the firm are critical to our understanding of training determinants.

\section{Limitations and Future Research}

Owing to the complexity of training decision-making in firms, Smith (2000) puts forth a compelling case for more case study research to inform future investigations. 
Nevertheless, common criticisms of case study research are researcher bias and lack of generalisability. The researcher has taken a number of precautions to prevent bias. Measures to enhance the study's validity and reliability include: data and source triangulation; developing a case study protocol; multi-case embedded case study design; and seeking feedback from participating case organisations on case drafts. The study's findings cannot be generalised to the wider population of India's IT sector or other sectors. In case study research, the focus is not on the generalisability of findings to the population; instead, the findings can be generalised or falsified according to the predictions of a theory.

One of the limitations of this research is the extent of transferability to other countries because the research is grounded in India's unique contextual environment and particularly in the strategic milieus of its IT firms. Nevertheless, some commonality in HR practices between Indian and overseas firms suggest the potential for transferability to other country contexts. For example, Organisations A, E, and G (all having overseas ownership) had similar approaches to training, quality and recruitment as Organisation F, an Indian-owned firm. Secondly, the timing of the study may have a bearing on future theory-building efforts. This study was undertaken at a time when the industry was witnessing high growth rates $(25-40 \%$ per annum). The explanations offered may not hold true when the growth rates decline and/or the industry begins to reel under pressure from an appreciating rupee, wage increases, the changing global geopolitical climate for offshoring and outsourcing, and more recently, the global credit crisis.

Since the data collection and analysis, the global economic outlook has worsened. One of the main industry sectors that the Indian IT sector was servicing-BFSI (Banking, Financial Services and Insurance) - has witnessed the collapse of major international banking and insurance firms. The immediate repercussions of the credit crisis are being felt by the sector, and there are early signs of revenue losses by some of the larger players whose exposure to the BFSI sector was high.

\section{Propositions for Future Research}

The propositions for future research that follow are premised on a dynamic and highgrowth outsourcing environment. The above changes in the global economic landscape offer a rich opportunity for comparison and development of rival explanations should 
the wider economic parameters of performance and trade change. Although this study is set in the Indian cultural context, the researcher believes that owing to the "born global" nature of this industry, the propositions for future research could be tested in similar high growth and dynamic services environments in different countries.

Future Research Proposition 1: ITeS/BPO firms will invest more in client-specific training than firms operating in ITSS.

Case findings from Organisations A, B, C, and D support the view that clientspecifications influence training provision. Organisations E, F, G, H, I, and J, however, demonstrate that client specifications have a relatively limited influence on training because application of information technology is generic and cuts across borders. This proposition has application for knowledge firms operating in business-to-business environments. Knowledge firms servicing multiple industry sectors, with differentiated services and a heterogeneous client base are more likely to invest in training because of differences in client specifications.

Future Research Proposition 2: Firms operating in slightly and highly differentiated markets and witnessing high growth rates will invest more in behavioural training than firms operating in mass service markets.

Service providers that are operating in value-added services often require frequent interactions with the client firm. The nature of the interactions and the need to develop managerial talent to foster organisational growth also necessitate investment in behavioural skills.

Future Research Proposition 3: Process and project specific training provision in dynamic and high-growth outsourcing services environments is not an integral part of a firm's human resource management infrastructure. Process and project-specific training is an inextricable part of operations.

Process and project-specific training was found to be intertwined with operations in the case organisations studied. This aspect needs to be explored in different cultural contexts. The nexus between operations and training will strengthen in establishing the legitimacy of HRD research. Only organisations that supported the development of 
leadership and management skills had some elements of such training as part of the HRM infrastructure. The nexus between operations and training was stronger in firms that had well-developed quality management systems. This finding gives rise to the next proposition.

Future Research Proposition 4: Firms in dynamic outsourcing environments that have well-developed quality management systems are more likely to have training and development integrated into their operations management functions than into their HRM infrastructure.

Case organisations found support for the above proposition as organisations' QMS, operations team, and process/project specific trainers worked collaboratively on assessing and delivering training and were structured outside the HRM infrastructure. HRM infrastructure was focusing more on the logistical arrangements of training.

Future Research Proposition 5: Outsourcing firms that have well-developed QMS and are following cost and differentiation strategies are likely to invest more in the nature and extent of training.

The case analysis amply demonstrated support for the above proposition. There are numerous professional service and knowledge firms operating in dynamic environments that offer fertile ground for testing this proposition.

Future Research Proposition 6: Firms operating in slightly and highly differentiated markets and having strong QMS will invest more in the training volume and diversity than firms competing only in mass service markets in dynamic outsourcing environments.

To accommodate changes in client specifications, legislation, and external labour markets - locally and globally - the variety and volume of training needed is high. This finding gives rise to a related proposition to examine the degree to which process or project complexity of services, instead of enterprise size, impacts on training volume and diversity. 
Future Research Proposition 7: High levels of business and process complexity rather than enterprise size, expressed in terms of number of employees, require high levels of training volume and diversity.

Organisations specialising in a narrow range and focusing on simple processes and low business complexity noted a reduced demand for training. On the contrary, intricate and complex processes and high overall business complexity required higher volumes and diversity of training provision.

Finally, the critical and unexplored synergistic relationship between an organisation's QMS and its LO, MO and, consequently, training gives rise to the last proposition of this thesis.

Future Research Proposition 8: The strength of an organisation's QMS influences the development of its learning and market orientation capabilities.

Although the above finding has been demonstrated in the case organisations and Figure 34, it is important to state that Figure 34 is a simple representation of relationships, that can be better uncovered through further research. Future research can use existing measurement scales for QMS, MO, and LO to test the impact on training provision and organisational performance. Ex-post facto research employing survey methodologies are suitable for testing the strength and direction of such relationships and for generalising the findings to the wider population.

\section{Summary and Conclusion}

Using case study as the research strategy, this thesis analysed the factors that influence provision of training in India's IT sector. Following within- and cross-case analyses, the main factors influencing training decision-making were uncovered and, a theoretical framework was developed for understanding training decision-making in IT firms in India's cultural context.

This concluding chapter discussed the implications for practice, policy and theory. Further, the study's limitations and propositions for future research were identified. Propositions for future research follow from the research findings and analysis. The 
findings suggest that training decision-making is complex and is shaped by the interaction of various factors - internal and external - in an organisation's strategic milieu and by the extent of dynamic coordination between clients and service provider and the organisational capabilities that they have developed over time. 


\section{REFERENCES}

Abramotvitz, M., \& Paul, A. D. (1996). Technological change and the rise of intangible investments: The U.S. economy's growth-path in the twentieth century. In OECD, Employment and growth in the knowledge-based economy (pp. 35-60). Paris: OECD.

Acemoglu, D., \& Pischke, J. (1998a). Why do firms train? Theory and evidence. Quarterly Journal of Economics, 113, 79-119.

Acemoglu, D., \& Pischke, J. (1998b). Beyond Becker: Training in imperfect markets. The Economic Journal, 109, 112-142.

Amit R., \& Schoemaker, P. J. (1993). Strategic assets and organizational rent. Strategic Management Journal, 14(1), 33-46.

Applebaum, E., Bailey, T., Berg, P., \& Kalleberg, A. L. (2000). Manufacturing advantage. Ithaca, NY: Cornell University Press.

Argyris C., \& Schon, D. A. (1978). Organizational learning: A theory of action perspective. Reading, MA: Addison-Wesley.

Arora, A. (2006). The Indian software industry and its prospects. The Heinz School of Public Policy and Management, Carnegie Mellon University.

Arora, A., Asundi, J. V., \& Fernandes, R. (2001). The Indian software services industry. Research Policy, 30(8), 1267-1287.

Arora, A., \& Athreye, S. (2002). The software industry and India's economic development. Information Economics and Policy, 14, 253-273.

Arora, A., Gambardella, A., \& Torrisi, S. (2001). In the footsteps of the Silicon Valley? Indian and Irish software in the international division of labour. [Discussion Paper No. 00-41] Stanford, CA: Stanford Institute for Economic Policy Research.

Arrow, K. (1973). Higher education as a filter. Journal of Public Economics, 2, 193-216.

Ashton, D., \& Green, F. (1996). Education, training and the global economy. Cheltenham, England: Edward Elgar.

Ashton, D., \& Sung, J. (2002). Supporting workplace learning for high performance working. Geneva, Switzerland: ILO.

Ashton, D., \& Sung, J. (2006). How competitive strategy matters? Understanding the drivers of training, learning and performance at the firm level (Research Paper No. 66). Oxford, England: Oxford and Warwick Universities, Centre for Skills, Knowledge and Organisational Performance.

Asundi, J., \& Arora, A. (2002, December). Quality certification and the economics of contract software development: A study of the Indian software industry (NBER 
Working Paper No. 7260). Pittsburgh, PA: H John Heinz III School of Public Policy and Management and Carnegie Mellon University.

Athreye, A. (2004). "The role of transnational corporations in the evolution of a high-tech industry: The case of India's software industry"-A comment. World Development, $32(3), 555-560$.

Athreye, A. (2005). The Indian software industry and its evolving service capability. Industrial and Corporate Change, 14(3), 393-418.

Audretsch, D. B., \& Feldman, M. P. (1996). Knowledge spillovers and the geography of innovation and production. American Economic Review, 86, 630-640.

Baird, L., \& Meshoulam, I. (1988). Managing two fits of strategic human resource management. Academy of Management Review, 13(1), 116-22.

Baker, W. E., \& Sinkula, J. M. (1999a). Learning orientation, market orientation, and innovation: Integrating and extending models of organisational performance. Journal of Market-Focussed Management, 4(4), 295-307.

Baker, W. E., \& Sinkula, J. M. (1999b). The synergistic effects of market orientation and learning orientation on organisational performance. Journal of the Academy of Marketing Science, 27(4), 411-427.

Baker, W. E., \& Sinkula, J. M. (2002). Market orientation, learning orientation and product innovation: Delving into the organisation's black box. Journal of Market-Focussed Management, 5(1), 5-23.

Baker, W. E., \& Sinkula, J. M. (2005). Market orientation and the new product paradox. Journal of Product Innovation Management, 22, 483-502.

Baker, W. E., \& Sinkula, J. M. (2007). Does market orientation facilitate balanced innovation programs? An organizational learning perspective. The Journal of Product Innovation Management, 24, 316-334.

Baldry, C., Bain, P., \& Taylor, P. (1998). "Bright satanic offices": Intensification, control and team Taylorism. In P. Thompson \& C. Warhurst (Eds.), Workplaces of the future (pp. 163-183). London: Macmillan Press.

Banerjee, P. (2004). The Indian software industry: Business strategy and dynamic coordination. New Delhi, India: Palgrave-MacMillan.

Banerjee, P. (2006). Strategies of outsourcing: From de-risking to outsourcing. In H. S. Kehal \& V. P. Singh (Eds.), Outsourcing and offshoring in the 21st century: A socioeconomic perspective. London: Idea Group.

Barney J. (1991). Firm resources and sustained competitive advantage. Journal of Management, 17(1), 99-120. 
Bartel, A. (2000). Measuring the employer's return on investments in training: Evidence from the literature. Industrial Relations, 39(3).

Bartel, A. P., \& Lichtenberg, F. R. (1987). The comparative advantage of educated workers in implementing new technology. The Review of Economics and Statistics, 69(1), 111.

Bartel, A. P., \& Sicherman, N. (1995). Technological change and skill acquisition of young workers (Working Paper No. 5107). Cambridge, MA: NBER.

Barrie, J., \& Pace, R. W. (1998). Learning for organizational effectiveness: Philosophy of education and human resource development. Human Resource Development Quarterly, 9, 39-54.

Barrie, J., \& Pace, R. W. (1999). Learning and performance: Just the end of the beginning-A rejoinder to Kuchinke. Human Resource Development Quarterly, 10, $293-296$.

Batt, R., \& Moynihan, L. (2002). The viability of alternative call centre production models. Human Resource Management Journal, 12(4), 14-34.

Becker, G. (1962). Investment in human capital: A theoretical analysis. Journal of Political Economy, 70, 9-49.

Becker, G. (1964). Human capital: A theoretical and empirical analysis Princeton, NJ: Princeton University Press.

Becker, B. E., \& Husled, M. A. (1998). High performance work systems and firm performance: A synthesis of research and managerial implications, Research in Personnel and Human Resource Management, 16, 53-101.

Beer, M., Spector, B., Lawrence, P. R., Quinn, M., \& Walton, R. E. (1984). Managing human assets. New York: The Free Press.

Belohlav, J. A. (1993). Quality, strategy, and competitiveness. California Management Review, 35(3), 55-69.

Benbasat, I., Goldstein, D. K., \& Mead, M. (1987). The case research strategy in studies of information systems. MIS Quarterly, 11(3), 159-182.

Benbasat, I., \& Zmud, R. (1999). Empirical research in IS: The practice of relevance. MIS Quarterly, 23(1), 3-16

Betcherman, G., Leckie, G., \& McMullen, K. (1997). Developing skills in the Canadian workplace, Ottawa, Canada: CPRN.

Bierema, L. L. (1997). Development of individuals leads to a more productive workplace. In R. Rowden (Ed.), Workplace learning: Debating five critical questions of theory and practice (pp. 21-28). San Francisco, CA: Jossey-Bass. 
Bing, J. W., Kehrhahn, M., \& Short, D. C. (2003). Challenges to the field of human resources development. Advances in Developing Human Resources, 5(3), 342-351.

Bishop, J. (1991). On the job training of new hires. In D. Stern \& J. Rizen (Eds.), Market failure in training? New economic analysis and evidence on training of adult employees. New York: Springer-Verlag.

Bishop, J. H. (1994). The incidence and payoff to employer training. New York: Cornell University, Centre for Advanced Human Resource Studies.

Boxall, P. (2003). HR strategy and competitive advantage in the service sector. Human Resource Management Journal, 13(3), 5-20.

Boxall, P., \& Purcell, P. (2003). Strategy and human resource management. New York: Palgrave Macmillan.

Boxall, P., \& Purcell, P. (2008). Strategy and human resource management. 2/e. New York: Palgrave Macmillan.

Braverman, H. (1974). Labor and monopoly capital: The degradation of work in the twentieth century. London: Monthly Review Press.

Bresnahan, T., Gambardella, A., \& Saxenian, A. (2001). Old economy' inputs for 'new economy' outcomes: Cluster formation in the new silicon valleys. Industrial and Corporate Change, 10(4), 835-860.

Breyfogle, F. W., III. (1999). Implementing Six Sigma: Smarter solutions using statistical methods. New York: Wiley.

Bright, J. R. (1958, July-August). Does automation raise skill requirements? Harvard Business Review., 36(4),85-98.

Brown, P., Ashton, D., Lauder, H., \& Tohlen, G. (2008). Towards a high-skilled, lowwaged workforce? A review of global trends in education, employment and labour market. Monograph No. 10, Centre on Skills, Knowledge, and Organisational Performance. Cardiff and Oxford Universities: Cardiff, UK.

Bryson, J., \& O’Neil, P. (2008, May). Developing human capability: Employment institutions, organisations and individuals. Discussion Paper. Wellington: Industrial Relations Centre, Victoria University of Wellington.

Burgess, J., \& Connell, J. (2004). Emerging developments in call centre research. Labour \& Industry, 14(3), 1-13.

Canadian Apprenticeship Forum. (2006). Return on apprenticeship training investment. Retrieved January 21, 2008 from http:/www.caffca.org/conf2k6/pres/SunJune4/CostROTI.pdf 
Cano, C. R., Carrillat, F. A., \& Jaramillo, F. (2004). A meta-analysis of the relationship between market orientation and business performance: Evidence from five continents. International Journal of Research in Marketing, 21, 179-200.

Cappelli, P., (1993). Are skills requirements rising? Evidence from production and clerical jobs. Industrial and Labour Relations Review, 46(3), 515-530.

Cappelli, P. (1994). Training and development in public and private policy. Brookfield, MA: Dartmouth.

Cappelli, P. (1995). Rethinking employment. British Journal of Industrial Relations, 33(4), $515-530$.

Cappelli, P., \& Rogovsky, N. (1994). New work systems and work requirements. International Labour Review, 133(2), 205-220.

Carton, P., Gannon, M., Jerrard, M., \& Shah, K. (2004). Matching the training program to the call centre model for best practice, International Employment Relations Review, 10(2), 85-106.

Caves, R. E. (1974). Multinational firms, competition and productivity in host-country markets. Economica, 41(162), 176-193.

Chang, C., \& Wang, Y. (1995). A framework for understanding differences in labor turnover and human capital investments. Journal of Economic Behaviour, 28, 91-105.

Chang, C., \& Wang, Y. (1996). Human capital investment under asymmetric information: The Pigovian conjecture revisited. Journal of Labour Economics, 16, 505-519.

Chermack, T. J., Lynham, S. A., \& Ruona, W. E. (2003). Critical uncertainities confronting human resource development. Advances in Developing Human Resources, 5(3), 257271.

Cohen, S. (1987). A labour process to nowhere? New Left Review, 165, 34-50.

Creswell, J. W. (2007). Qualitative inquiry and research design: Choosing among five approaches. London: Sage.

Davis, G. F., McAdam, D., Scott, W. R., \& Zald, M. N. (Eds.). (2005). Social movements and organization theory, New York: Cambridge University Press.

Dawe, S. (2003). Determinants of successful training practices in large Australian firms. Leabrook, SA: NCVER.

Day, G. S. (1994). The capabilities of market-driven organizations. Journal of Marketing, 58(October), 37-52.

De Cieri, H., \& Holland, P. (2006). The strategic role of human resource development. In P. $\&$ H. De Cieri (Eds.), Contemporary issues in human resource development: Holland (pp. 3-24). Frenchs Forest, NSW: Pearson Education Australia. 
de Geus, A. P. (1988, March/April). Planning as learning. Harvard Business Review, 66, $70-74$.

de Menezes, L. M., \& Wood, S. (2006). The reality of flexible work systems in Britain. International Journal of Human Resource Management, 17(1), 106-138.

Dean, J. W., Jr., \& Snell, S. A. (1991). Integrated manufacturing and job design: Moderating effects of organisational inertia. Academy of Management Journal, 34(4), 776-804.

Dearden, L., Reed, H., \& Van Reenen, J. (2006). The impact of training on productivity and wages: Evidence from British panel data. Oxford Bulletin of Economic and Social Research, 68(4), 397-421.

Denzin, N. K., \& Lincoln, Y. S. (2005). The Sage handbook of qualitative research. Thousand Oaks, CA: Sage.

Deshpande, R., \& Webster, F. E. (1989, Fall). Organizational culture and marketing: Defining the research agenda. Journal of Marketing, 47, 101-110.

Deshpande, R., John U. F., \& Webster, F. E. (1993, January). Corporate culture, customer orientation, and innovativeness in Japanese firms: A quadrad analysis. Journal of Marketing, 57(1), 23-37.

Dickson, P. (1996, October). The static and dynamic mechanics of competition: A comment on Hunt and Morgan's competitive advantage theory. Journal of Marketing, 60, 102106.

Dion, R. (1993, July). Process improvement and the corporate balance sheet, IEEE Software, 28-35.

Dirkx, J. M. (1997). Human resource development as adult education: Fostering an educative workplace. In R. Rowden (Ed.), Workplace learning: Debating five critical questions of theory and practice (pp. 41-47). San Francisco, CA: Jossey-Bass.

Dooley, L. M. (2002, August). Case study research and theory building. Advances in Developing Human Resources, 4, 335-354.

Dossani, R. (2005). Origins and growth of the software industry in India (Working paper). Palo Alto, CA: Stanford University, Asia-Pacific Research Centre.

Dostie, B., \& Montmarquette, C. (2007). Employer sponsored training in Canada: Synthesis of using data from the workplace and employee survey. Quebec, Canada: Human Resources and Social Development Canada.

Dossani, R. (2005). Origins and growth of the software industry in India (Working paper). Palo Alto, CA: Stanford University, Asia-Pacific Research Centre. 
DTI. (2003). Innovation report-Competing in the global economy: The innovation challenge. London: Department of Trade and Industry.

Dyer, L. (1984). Studying human resource strategy: An approach and an agenda. Industrial Relations, 23(2), 156-169.

Dube, L., \& Pare, G. (2003). Rigor in IS positivist case study research: Current practices, trends, and recommendations. MIS Quarterly, 27(4), 597-635.

Dubin, R. (1978). Theory building. New York: New Free Press.

Economic Times. (2007, March). \$2bn spent for IT/ITeS Co's on training in '07. Retrieved April 2, 2007, from www.economictimes.com

Edwards, P. K. (1995). From industrial relations to the employment relationship: The development of research in Britain. Relations Industrielles, 50(1), 39-63.

Eisenhardt, K. M. (1989). Building theories from case study research. Academy of Management Review, 14(4), 532-550.

Eisenhardt, K. M., \& Martin, J. A. (2000). Dynamic capabilities: What are they? Strategic Management Journal, 21, 1504-1511.

Ethiraj, S. E., Kale, P., Krishnan, M. S., \& Singh, J. V. (2005). Where do capabilities come from and how do they matter? A study in the software services industry. Strategic Management Journal, 26, 25-45.

Fazzari, A. J., \& Levitt, K. (2008). Human resources as a strategic partner: Sitting at the table with Six Sigma. Human Resource Development Quarterly, 19(2), 171-180.

Felstead, A., \& Ashton, D. (2000). Tracing the link: Organisational structures and skill demands. Human Resource Management Journal, 10(3), 5-21.

Felstead, A., \& Gallie, D. (2002). For better or worse? Non-standard jobs and high involvement work systems (SKOPE Research Paper No. 29). Oxford, England: Oxford and Warwick Universities, SCOPE.

Felstead, A., Gallie, D., \& Green, F. (2002). Work skills In Britain 1986-2001. Nottingham, England: DfES.

Felstead, A., \& Green, F. (1994). Cycles of training? Evidence from the British recession of the early 1990s. In A. Booth \& D. Snower (Eds.), The skills gap and economic activity. Cambridge, England: Cambridge University Press.

Fernie, S., \& Metcalfe, D. (1998). Not hanging on the telephone: Payment systems in the new sweatshops (Discussion Paper No. 390). London: London School of Economics.

Finegold, D. (1991). Institutional incentives and skill creation: Preconditions for high skills equilibrium. In P. Ryan (Ed.), International comparisons of vocational education and training for intermediate skills. London: Falmer. 
Finegold, D. (1992). The changing international economy and its impact on education and training. Oxford Studies in Comparative Education, 2(2), 57-82.

Finegold, D. (1999). Creating self-sustaining, high-skill ecosystems. Oxford Review of Economic Policy, 15(1), 60-72.

Finegold, D., \& Soskice, D. (1988). The failure of British training: Analysis and prescriptions. Oxford Review of Economic Policy, 4, 21-53.

Fiol, C. M., \& Lyles, M. A. (1985). Organisational learning. Academy of Management Review, 10(4), 656-670.

Flyvberg, B. (2006). Five misunderstandings about case study research. Qualitative Inquiry, 12(2), 219-245.

Fombrun, C. J., Tichy, N. M., \& Devanna, M. A. (1984). Strategic human resource management. New York: Wiley.

Gallie, D. (1991). Patterns of skill change: Upskilling, deskilling or polarisation of skills? Work, Employment and Society, 5(3), 319-51.

Garavan, T. (1997). Training, development, education, and learning: Different or the same? Journal of European Industrial Training, 21(2), 39-50.

Garavan, T. N., Gunnigle, P., \& Morley, M. (2000). Contemporary HRD research: A triarchy of theoretical perspectives and their prescriptions. Journal of European Industrial Training, 24(1-4), 65-93.

Garvin, D. A. (1993, September/October). Building a learning organization. Harvard Business Review, 71, 53-58.

Githens, R. P., Dirani, K., Gitonga, J., \& Teng, Y. (2008). Technology-related research in HRD publications: an analysis of content and metaperspectives from 2000 to 2006 . Human Resource Development Quarterly,19(3), 191-216.

Gorg, H., \& Strobl, E. (2002a). Multinational companies and indigenous development: An empirical analysis. European Economic Review, 46, 1305-1322.

Gorg, H., \& Strobl, E. (2002b). Spillovers from foreign firms through worker mobility: An empirical analysis (Discussion Paper No. 591). Bonn, Germany: IZA.

Green, F., Mayhew, K., \& Molloy, E. (2003). Employers’ perspectives survey, 2000. Sheffield, England: Department for Education and Skills.

Groot, W. (1997). Enterprise related training: A survey. Melbourne, Australia: Monash University, ACER.

Guest, D. (2000, July). HR and the bottom Line: 'Has the penny dropped?'. People Management, 20, 26-31. 
Guest, D. (2001). Human resource management: When research confronts theory. International Journal of Human Resource Management, 12(7), 1092-1106.

Guest, D., Michie, J., Conway, N., \& Sheehan, M. (2003). Human resource management and corporate performance. British Journal of Industrial Relations, 41(2), 291-315.

Hall, R., Bretherton, T., \& Buchanan, J. (2000). It's not my problem: The growth of nonstandard work and its impact on vocational education and training in Australia.

Hamel, G., \& Prahalad, C. K. (1990, May-June). The core competences of the corporation. Harvard Business Review, 79-91.

Hamlin, R. (2007). An evidence based perspective on HRD. Advances in Developing Human Resources, 9(1), 42-57.

Harrison, R., \& Kessels. J. (2004). Human resource development in a knowledge economy. New York: Palgrave McMillan.

Harter, D. E., Krishnan, M. S., \& Slaughter, S. A. (2000). Effects of process maturity on quality, cycle time and effort in software development. Management Science, 46(4), $451-467$.

Hatcher. T. (2000). A Study of the Influence of the Theoretical Foundations of Human Resource Development on Research and Practice. Presented at the Academy of Human Resource Development International Conference, Research Triangle, N.C.: AHRD.

Hayton, G., McIntyre, J., Smart, R., McDonald, R., Noble, C., Smith, A., et al. (1996). Final report: Enterprise training in Australia, Melbourne, Australia: Office of Training and Further Education.

Heeks, R., (1996), Indian software industry: State policy, liberalisation and industrial development, New Delhi, India: Sage.

Heeks, R. (1998, October). The uneven profile of Indian software exports (Working Paper). Manchester, England: University of Manchester, IDPM. Retrieved January 27, 2009, from http://www.man.ac.uk/idpm

Hendry, C. (1991). Training and corporate strategy. In J. Stevens, \& R. MacKay (Eds.), Training and competitiveness. London: Kogan Page.

Herzenberg, S., Alic, J., \& Wial, H. (1998). New rules for a new economy: Employment and opportunity in postindustrial America, Ithaca, NY: ILR Press.

Heyes, J., \& Stuart, M. (1996). Does training matter? Employee experiences and attitudes. Human Resource Management Journal, 6(3), 7-21.

Hill, C. W. L. (1988). Differentiation versus low cost or differentiation and low cost: A contingency framework. Academy of Management Review, 13(3), 401-412. 
Holton, E. F. (2002). Theoretical assumptions underlying performance paradigm of human resource development. Human Resource Development International, 5(2), 199-215.

Holton, E. F. (2004). Implementing evidence-based practices: Time for a national movement? Human Resource Development Review, 3(3), 187-188.

Huber, G. P. (1991, February). Organizational learning: The contributing processes and the literatures. Organization Science, 2, 88-115.

Humphrey, W. S., Snyder, T. R., \& Willis, R. R. (1991, July). Software process improvement at Hughes Aircraft. IEEE Software, 11-23.

Hunt, S. D. (1994). On re-thinking marketing: Our discipline, our practice, our methods. European Journal of Marketing, 23(3), 13-25.

Hunt, S. D., \& Morgan, R. M. (1996, October). The resource-advantage theory of competition: Dynamics, dependencies, and evolutionary dimensions. Journal of Marketing, 60, 107-114.

Hutchinson, S., Purcell, J., \& Kinnie, N. (2001). Evolving high commitment management and the experience of the RAC call centre. Human Resources Management Journal, 10(1), 63-78.

ILO. (2001). World employment report 2001: Life at work in the information economy. Geneva, Switzerland: Author.

Kaplan, D. M. \& Lerouge, C. (2007). Managing on the edge of change: human resource management of information technology employees. Human Resource Management, 46(3), 325-330.

Kaplan, B., \& Maxwell, J. A. (1994). Qualitative research methods for evaluating computer information systems. In J. G. Anderson, C. E. Aydin, \& S. J. Jay (Eds.), Evaluating health care information systems: Methods and applications (pp. 45-68). Thousand Oaks, CA: Sage.

Katz, E., \& Ziderman, A. (1990). Investment in general training: The role of information and labour mobility. Economic Journal, C, 1478-1158.

Keep, E. (1999). UK's VET policy and the 'Third Way': Following a high skills

trajectory or running up a dead end street? Journal of Education and Work, 12(3), 323-346.

Keep, E. (2000). Upskilling Scotland. Edinburgh, Scotland: Centre for Scottish Public Policy.

Keep, E., \& Mayhew, K. (1996). Towards a learning society: Definition and measurement. Policy Studies, 17(3), 215-232.

Ketter, P. (2006). Investing in learning: Looking for performance, $T+D, 60(12)$. 
Kirkpatrick, D. L. (1998). Evaluating training programs: The four levels. (2nd ed.). San Francisco, CA: Berrett-Koehler.

Kitchin, J., \& Blackburn, R. (2002). The nature of training and motivation to train in small firms (Research Report No. RR330). Nottingham: Department for Education and Skills.

Knowles, M. S., Holton, E. F., III., \& Swanson, R.A. (1998). The adult learner: The definitive classic in adult education and human resource development (5th ed.). Houston, Texas: Gulf Publishing Company.

Kochan, T., Katz, T., \& McKersie, R. (1986). The transformation of American industrial relations. New York: Basic Books.

Kohli, A. K., \& Jaworski, B. J. (1990, April). Market orientation: The construct, research propositions, and managerial implications. Journal of Marketing, 54, 1-18.

Kohli, A. K., Jaworski, B. J., \& Kumar, A. (1993, November). MARKOR: A measure of market orientation. Journal of Marketing Research, 30, 467-477.

Krishnan, M. S., Kriebel, C. H., Kekre, S., \& Mukhopadhyay, T. (2000, June). An empirical analysis of productivity and quality in software products. Management Science, 46(6), $745-759$

Kuckinke, P. K., (2001). Why HRD is not an academic discipline. Human Resource Development International, 4(3), 291-94.

Kumar, N. (2001). Developing countries in international division of labour in software and service industry: Lessons from the Indian experience. World employment report 2001: Life at work in the information economy [CD-ROM]. Geneva: ILO.

Kumar, P. (2000). Rethinking high-performance work systems (Current issues series). Kingston, Ontario, Canada: Queen's University, Industrial Relations Centre.

Lazear, E. P., \& Moore, R. L. (1984). Incentives, productivity and labour contracts. Quarterly Journal of Economics, LXXVII, 275-295.

Lee, A. S. (1989). A scientific methodology for MIS case studies. MIS Quarterly, 13(1), $33-52$.

Lee, M. (2001). A refusal to define HRD. Human Resource Development International, 3(4), 327-341.

Leimbach, M. P., \& Baldwin, T. T. (1997). How research contributes to the HRD value chain, In R. A. Swanson \& E. F. Holton, III (Eds.), Human resource development: A research handbook. San Francisco, CA: Berrett-Koehler.

Leonard, D. (1998). Wellsprings of knowledge: Building and sustaining sources of innovation. Boston, MA: Harvard Business School Press. 
Lepak, D. P., \& Snell, S. A. (2003). Managing the human resource architecture for knowledge-based competition. In S. E. Jackson, H. A. Hitt, \& A. S. DeNisi (Eds.), Managing knowledge for sustained competitive advantage (pp. 127-154). San Fransisco, CA: Jossey-Bass.

Lincoln, Y. S., \& Guba, E. G. (2000). Paradigmatic controversies, Contradictions, and emerging confluences. In N. K. Denzin \& Y. S. Lincoln, The Sage handbook of qualitative research (2nd ed., pp. 163-187). Thousand Oaks, CA: Sage.

Lloyd, C., \& Payne, J. (2004). Just another bandwagon? A critical look at the role of the high performance workplace as a vehicle for the UK high skills projec. (SKOPE Working Paper No. 49). Oxford, England: Oxford and Warwick Universities, SKOPE. Lloyd, C., \& Payne, J. (2005). High performance work organisation: A driver for the high skills vision? (SKOPE Working Paper No. 49). Oxford, England: Oxford and Warwick Universities, SKOPE

Lynch, L. M. (1991). The role of off-the-job vs. on-the-job training for the mobility of women workers. American Economic Review, 81(2), 151-56.

Lynham, S. A. (2000). Theory building in the human resource development profession. Human Resource Development Quarterly, 11(2), 159-178.

Lynham, S. A. (2002). The general method of theory building research in applied disciplines. Advances in Developing Human Resources, 4(3), 221-241.

Maglen, L. (1990, December). Challenging the human capital orthodoxy: The educationproductivity link re-examined. The Economic Record, 281-294.

Malik, A. (2009). Training drivers, competitive strategy and clients' needs: Case studies of three business process outsourcing organisations. Journal of European Industrial Training, 33(2), 160-177.

Malik, A., \& Nilakant, V. (2008). What drives training in India's IT-enabled and business process outsourcing industry? Visual presentation at the 2008 Academy of Management Meeting, Anaheim, USA.

Mankin, D. (2001). A model for human resource development. Human Resource Development International, 4(1), 65-86.

Marchington, M., \& Grugulis, I. (2000). "Best practice" human resource management: Perfect opportunity or dangerous illusion?'. International Journal of Human Resource Management, 11(6), 1104-1124.

Marshall, A. (1920). Principles of economics (8th ed.). London: Macmillan.

Mason, G. (2005, May). In search of high value added production: How important are skills? [Research Report No. 663] Nottingham: Department for Education and Skills. 
Mason, G. (2004). Enterprise product strategies and employer demand for skills in Britain: Evidence from the employers' skill surveys (SKOPE Working Paper No. 50). Warwick, England: University of Warwick.

May, G. L., Sherlock, J. J., \& Marby, C. K. (2003). Drive for shareholder value and potential implications for HRD. Advances in Developing Human Resources 5(3), 321331.

McGoldrick, J., Stewart, J., \& Watson, S. (2001). Theorising human resource development, Human Resource Development International, 4(3), 343-356.

McIntyre, J., Petocz, P., Hayton, G., Smith, A., \& Roberts, P. (1996). Enterprise training in Australia: National survey of training. Melbourne, Australia: Office of Training and Further Education.

McLaglan, P. A. (1989). The models: A volume of models for HRD practice. Alexandria, VA: American Society for Training and Development.

McLean, G. N. (1998). HRD: A three-legged stool, an octopus or a centipede? Human Resource Development International, 1(4), 375-377.

McLean, G. N. (1999). Get out the drill, glue and more legs. Human Resource Development International, 2(1), 6-7.

McLean, G. A. (2001). Contrasting three modes of inquiry. Unpublished manuscript. McLean, G. N., \& McLean, L. (2001). If we can't define HRD in one country, how can we define it in an international context? Human Resource Development International, 4(3), 313-326.

McNabb, R., \& Whitfield, K (1994). The market for training: An overview. In R. McNabb $\& \mathrm{~K}$. Whitfield (Eds.), The market for training: International perspectives on theory, policy and methodology (pp. 1-18). Aldershot, England: Avebury.

MHRD. (2007). Selected educational statistics-2004-05. New Delhi, India: Ministry of Human Resource Development, Department of Higher Education, Statistics Division.

Miles, B. M., and Huberman, A. M. (1994). Qualitative data analysis: An expanded sourcebook (2nd ed.). Thousand Oaks, CA: Sage.

Mincer, J. (1974). Schooling, experience and earnings. New York: Columbia University Press.

MIT. (2002). Study team report on human resource development for 10th Five Year Plan2002-2007. New Delhi, India: Ministry Of Information Technology.

Morgan, N. A., \& Piercy, N. F. (1996). Competitive advantage, quality strategy, and the role of marketing. British Journal of Management, 7(3), 231-245. 
Nafukho, F. M., Hairston, N. R., \& Brooks, K. (2004). Human capital theory: Implications for human resource development. Human Resource Development International, 7(4), $545-551$.

Narayanan, R., \& Neethi, S. (2005). Creating human resources for information technology: A systemic study (Report submitted to NASSCOM IT Workforce Development Group, Version 2). Trivandrum, India: Tata Consultancy Services.

Narver, J. C., \& Slater, S. F. (1990, October). The effect of a market orientation on business profitability. Journal of Marketing, 54, 20-35.

Narver, J. C., Slater, S. F., MacLachlan, D. L. (2004). Responsive and proactive market orientation. Product Development \& Management Association, 21, 334-347.

NASSCOM. (2005a). Key statistics: ITeS/BPO sector. Retrieved January 7, 2005, from www.nasscom.org

NASSCOM. (2005b). Key statistics: IT sector. Retrieved January 7, 2005, from Www.nasscom.org

NASSCOM (2006a). Why India?. Retrieved July 12, 2006, from www.nasscom.org

NASSCOM (2006b). Knowledge professionals. Retrieved July 12, 2006, from www.nasscom.org

NASSCOM (2007). IT industry factsheet: August 07. Retrieved September 25, 2007, from www.nasscom.org

NASSCOM \& KPMG. (2004). Strengthening the human resource foundations of the Indian IT enabled services/IT Industry. New Delhi, India: NASSCOM.

NASSCOM-McKinsey. (2005). NASSCOM-McKinsey Report 2005. New Delhi, India: NASSCOM.

Nelson, R. R. (1991, Winter). Why do firms differ, and how does it matter? Strategic Management Journal, 12, 61-74.

Nelson, R., \& Phelps, E. (1966). Investment in humans, technological diffusion, and economic growth. American Economic Review, 56, 69-75.

Nelson, R., Peck, M., \& Kalacheck, E. (1967). Technology, economic growth, and public policy. Washington, DC: Brookings.

Nelson, R. R., \& Winter, S. (1982). An evolutionary theory of economic change. Cambridge, MA: Belknap Press.

Nilakant, V. (2005, August). Institutional dynamics in the evolution of the Indian software industry. Paper presented at the Academy of Management Conference, Hawaii, U.S.A. 
NTITSD. (2003). Task force on meeting the human resource challenge for IT and IT enabled services: Report and recommendations. New Delhi, India: Department of Information Technology, Ministry of Communication and Information Technology, Government of India.

OECD. (1994). The OECD jobs study: Evidence and explanations. Paris: Author.

OECD/CERI. (1986). New technology and HRD in automobile industry. Paris: OECD.

OECD/CERI. (1988). Human resources and corporate strategy: Technology change in banks and insurance companies. Paris: OECD.

Osterman, P. (1994). How common is workplace transformation and who adopts it? Industrial and Labour Relations Review. 47(2), 173-188.

Osterman, P. (1995). Skills, training, and work organisation in American establishments. Industrial Relations, 34(2), 125-46.

Ottosson, S., \& Wang, W. (1997). Product life cycles and continuing education implications. European Journal of Engineering Education, 22(4), 427-343.

Packar, A. H., \& Sharrar, G. K. (2003). Lifelong learning, corporate social responsibility and the changing nature of work. Advances in Developing Human Resources, 5(3), $332-341$.

Pare, G. (2004). Investigating information systems with positivist case study research. Communications of the Association for Information Systems, 13, 233-264.

Parzinger, M. (1997). A stage-wise application of total quality management through the product life cycle. Industrial Management \& Data Systems, 97(3), 125-130.

Patibandla, M., \& Petersen, B. (2002). Role of transnational corporations in the evolution of a high-tech industry: The case of India's software industry. World Development, 30(9), 1561-1577.

Patton, M. Q. (2002). Qualitative evaluation and research methods (3rd ed.). Newbury Park, CA: Sage.

Paulk, M. C., Curtis, B., Chrissin, M. B., \& Weber, C. V. (1993). Capability maturity model, Version 1.1. IEEE Software, 10(4), 18-27.

Prajogo, D. I. (2007). The relationship between competitive strategies and product quality. Industrial Management \& Data Systems,107(1), 69-83.

Prajogo, D. I. \& Brown, A. (2004). The relationship between TQM practices and quality performance and the role of formal TQM programs: An Australian empirical study. The Quality Management Journal, 11(4), 31-42. 
Prajogo, D. I., \& McDermott, C. M. (2006). The relationship between total quality management and organizational culture. International Journal of Operations \& Production Management, 25(11), 1101-1122.

Penrose, E. T. (1959). The theory of the growth of the firm. New York: Wiley.

Peteraf, M., \& Shanley, M. (1997). Getting to know you: A theory of strategic group identity. Strategic Management Journal, 18(S), 165-186.

Pettigrew, A., Sparrow, P., \& Hendry, C. (1988, December). The forces that trigger training. Personnel Management, 28-32.

Pfeffer, J. (1994). Competitive advantage through people: Unleashing the power of the workforce. Boston, MA: Harvard Business School Press.

Pfeffer, J. (1998). The human equation: Building profits by putting people first. Boston, MA: Harvard Business School Press.

Porter, M. (1985). Competitive advantage: Creating and sustaining superior performance. New York: Free Press.

Porter, M. E., Schwab, K., Sala-I-Martin, X., \& Lopez-Claros, A. (2004). The global competitiveness report. Geneva, Switzerland: World Economic Forum.

Purcell, J. (1999). The search for "best practice" and "best fit": Chimera or cul-de-sac. Human Resource Management Journal 9(3), 26-41.

Purcell, J., Kinnie, N., Hutchinson, S., Rayton, B., \& Swart, J. (2003). Understanding the people and performance link: Unlocking the black box. London: CIPD.

Radice, R. (1995). ISO9001 interpreted for software organizations. Andover, MA: Paradoxicon.

Rainbird, H. (1994). Continuing training. In K. Sission (Ed.), Personnel Management (2nd ed). Oxford, England: Blackwell.

Rao, T. V. (2004). Human resource development as national policy in India. Advances in Developing Human Resources, 6(3), 288-296.

Rao, T. V., \& Abhraham, E. (1986). HRD practices in Indian industries: A trend report. Management and Labour Studies, 2, 73-85.

Rao, T. V. \& Varghese, S. (2009). Trends and challenges of developing human capital in India. Human Resource Development International,12(1), 15-34.

Reed, R., Lemak, D. J., \& Mero, N. P. (2000). Total quality management and sustainable competitive advantage. Journal of Quality Management, 5, 5-26.

Reed, R., Lemak, D. J., \& Montgomery, J. C. (1996). Beyond process: TQM content and firm performance. Academy of Management Review, 21, 173-202. 
Richards, L., \& Morse, J. M. (2007). Read me first for a user's guide to qualitative methods. London: Sage.

Ridoutt, L., Dutneall, R., Hummel, K., \& Smith, A. (2002). Factors influencing training and learning in the workplace. Leabrook, SA: NCVER.

Ritzer, G. (2004). The McDonaldisation of Society: Revised new century edition. California: Pine Forge Press.

Rouna, W. E., Lynham, S. A., \& Chermack, T. J. (2003). Insights on emerging trends and the future of human resource development. Advances in Developing Human Resources, 5(3), 272-282.

Russell, C. (1999). Innovation and quality. The Quality Magazine, 8(4), 51-52.

Sambrook, S., \& Stewart, J. (1998). Talking of HRD. Human Resource Development International, 3(2), 159-78.

Saxenian, A. (1994). Regional advantage, culture and competition in Silicon Valley and Route 128. Cambridge, MA: Harvard University Press.

Saxenian, A. (2001). Bangalore: The Silicon Valley of Asia? (Working Paper No. 91). Palo Alto, CA: Stanford University, Center for Research on Economic Development and Policy Reform.

Schuler R. S., \& Jackson, S. E. (1997). Linking competitive strategies with human resource management practices. Academy of Management Executive, 1(3), 209-213.

Scott, W. R., Ruef, M., Mendel, P. J., \& Caronna, C. A. (2000). Institutional change and healthcare organizations: From professional dominance to managed care. Chicago, IL: The University of Chicago Press.

Senge, P. (1990). The fifth discipline: The art and practice of learning organisation. New York: Doubleday.

Shah, K., \& Jerrard, M. (2004). Searching best practice training programs in Indian call centres: A pilot study. In G. Elkin (Ed.), Proceedings of the $18^{\text {th }}$ Annual Conference of the Australian and New Zealand Academy of Management (ANZAM). Dunedin, New Zealand: University of Otago.

Short, D. (2006). The gap between research and practice in HRD: A summary of the discussion 1995-2005. In Proceedings of the 2006 International Academy of HRD Conference. Bowling Green, OH: AHRD.

Shultz, T. (1961, March). Investment in human capital. American Economic Review, 1-17.

Singh, S. (2003, November 10). Infotec: Home run. Business World. Retrieved 26 January 2005 www.businessworldindia.com/Nov1003/coverstory01.asp. 
Sinkula, J. M. (1994, January). Market information processing and organisational learning. Journal of Marketing, 58, 46-55.

Sinkula, J. M. (2002). Market-based success, organisational routines, and unlearning. Journal of Business and Industrial Marketing, 17(4), 253-269.

Sinkula, J. M., Baker, W. E., \& Noordeweir, T. (1997). A framework for market-based organisational learning: Linking values, knowledge, and behaviour. Journal of the Academy of Marketing Science, 25, 305-318.

Sitkin, S. B., Sutcliffe, K. M., \& Schroeder, R. G. (1994). Distinguishing control from learning in total quality management: A contingency perspective. Academy of Management Review, 19(3), 537-564.

Slater, S. F., \& Narver, J. C. (1995, July). Market orientation and the learning organization. Journal of Marketing. 59, 63-74.

Smith, A. (2000). Casing the joint: Case study methodology in VET research at the organisational level. Australian and New Zealand Journal of Vocational Education Research, 8(1), 73-91.

Smith, A., \& Dowling, P. J. (2001). Analysing firm training: Five propositions for future research. Human Resource Development Quarterly, 12(2), 147-167.

Smith, A., \& Hayton, G. (1999). What drives enterprise training? Evidence from Australia. The International Journal of Human Resource Management, 10(2), 251272.

Smith, A., Oczkowksi, E., Macklin, R., \& Noble, C. (2004). The impact of organisational change on the nature and extent of training in Australian enterprises. International Journal of Training and Development, 8(2), 2-15.

Smith, A., Oczkowksi, E., Noble, C., \& Macklin, R. (2003). Organisational change and management of training in Australian enterprises. International Journal of Training and Development, 7(1), 94-110.

Smith, A., Burke, G., Long, M., \& Dumbrell, T. (2008). Approaches to measuring and understanding employer training expenditure. Adelaide, SA: NCVER.

Snell, S. A., \& Dean, J. W. (1992). Integrated manufacturing and human resource management: A human capital perspective. Academy of Management Journal, 35(3), 467-504.

Sparrow, J., \& Pettigrew, A. (1985). Britain's training problems: The search for a strategic HRM approach. Human Resource Management, 26(1), 109-127.

Spence, M. (1973). Job market signalling. Quarterly Journal of Economics, LXXXVII, 355375. 
Steedman, H., \& Wagner, K. (1987, November). A second look at productivity, machinery and skills in Britain and Germany. National Institute Economic Review, 84-95.

Stake, R. (1995). The art of case study research. London: Sage.

Stewart, J., \& Mavondo, F. (2005). Organisational learning profiles and their effect on market orientation, innovation and performance. In Proceedings of the 2005 British Academy of Management Conference. Oxford, England: University of Oxford.

Storey, J. (1989). New perspectives in human resource management. London: Routledge.

Strober, M. (1990). Human capital theory: Implications for HR managers. Industrial Relations, 29(2), 214-239.

Subramanian, C. R. (1992). India and the computer: A study of planned development. NewDelhi, India: Oxford University Press.

Sung, J., \& Ashton, D. (2005). Achieving best practice in your business: High performance work practices: Linking strategy and skills to performance outcomes. London: DTI

Swanson, R. A. (1997). TADDS short (theory application deficit disorder). Human Resource Development Quarterly, 8(3), 193-195.

Swanson, R. A. (1999). HRD theory: Real or imagined? Human Resource Development International, 2(1), 2-5.

Swanson, R. A. (2001). Human resource development and its underlying theory. Human Resource Development International, 4(3), 299-312.

Swanson, R. (2007). Theory framework for applied disciplines: Boundaries, contributing, core, useful, novel, and irrelevant components. Human Resource Development Review, 6(3), 321-339.

Swanson, R. A., \& Holton, E. F., III. (1997). Human resource development research handbook. San Fransisco, CA: Berrett-Koehler.

Swanson, R. A., \& Holton, E. F., III. (2001). Foundations of human resource development. San Fransisco, CA: Berrett-Koehler.

Swanson, R. A., Lynham, S. A., Rouna, W. E., \& Torraco, R. J. (2000). Theory building researching HRD: Pushing the envelope! In Proceedings of the Academy of Human Resource Development, Research Triangle, NC: AHRD.

Taylor, S. (1998). Emotional labour and the new workplace. In P. Thompson \& C. Warhurst (Eds.), Workplaces of the future. London: Macmillan.

Taylor, P., \& Bain, P. (1999). "An assembly line in the head": Work and employee relations in the call centre. Industrial Relations Journal, 30(2), 101-117.

Taylor, P., \& Bain, P. (2001). Trade unions, workers rights and the frontier of control in UK call centres. Economic and Industrial Democracy, 22(1), 39-66. 
Taylor, P., \& Bain, P. (2004, April). Call centre offshoring to India: The revenge of history? Labour \& Industry, 14(3), 15-24.

Taylor, S., \& Patton, R. (2002). Corporate universities-historical development, conceptual analysis and relations with public sector higher education. London: The Observatory on Borderless Higher Education.

Teece, D. J., Pisano, G., \& Schuen, A. (1997). Dynamic capabilities and strategic management. Strategic Management Journal, 18, 509-533.

Turcotte, J., \& Rennison, L. W. (2004, Fall). Productivity and wages: Measuring the effect of human capital and technology use from linked employer-employee data. International Productivity Monitor, (9), 25-36.

VandenHeuvel, A., \& Wooden, M. (1999). Casualisation and outsourcing: Trends and implications for work-related training. Adelaide, Australia: NCVER.

van den Broek, D. (2002). Monitoring and surveillance in call centres: Some responses from Australian workers. Labour \& Industry, 12(3), 43-58.

Vogel, N. (2000, September). "Dispelling the myth". Momentum, 2, 12-19.

Walton, J. (1999). Strategic human resource development. Harlow, Canada: Financial Times/Prentice Hall.

Walton, J. (1999a). Human resource development and the corporate university. In J. Walton, Strategic human resource development. London: Pearson Education.

Walton, R. E. (1985). From control to commitment in the workplace. Harvard Business Review, 63(2), 77-84.

Wang, G. G. (2003). On the two-way customer service model in HRD. Human Resource Development Review, 2(4), 453-459.

Watkins, K., \& Marsick, V. (1992). Towards a theory of informal and incidental learning in organisations, International Journal of Lifelong Education, 11(2), 287-300.

Weick, K. E. (1979). Engaged scholarship: Creating knowledge for science and practice. Cambridge, England: Oxford University Press.

Welch, F. (1970). Education in production. Journal of Political Economy, 78, 35-59.

Wernerfelt, B. (1984). A resource-based view of the firm. Strategic Management Journal, $5,795-815$.

Willis, V. J. (1997). HRD as evolutionary system: From pyramid building to space walking around. In Proceedings of the Academy of Human Resource Development, Atlanta, GA: Atlanta, GA. Academy of Human Resource Development.

Wilkinson, F. (1983). Productive systems. Cambridge Journal of Economics, 7, 413-429.

Wilkinson, F. (2002). Productive systems and the structuring role of economic and social 
theories (Working Paper No. 225). Cambridge, England: University of Cambridge, ESRC Centre for Business Research.

Winter, S. G. (2003). Understanding dynamic capabilities. Strategic Management Journal, 24(10), 991-995.

Wright, P. M., \& McMahan, G. C. (1992). Theoretical perspectives for strategic human resource management. Journal of Management , 18(2), 295-320.

Wright, P. M., McMahan, G. C., \& McWilliams, A. (1994). Human resources and sustained competitive advantage: A resource-based perspective. The International Journal of Human Resource Management, 5(2), 301-326.

Womack, J. J., Jones, D., \& Roos, D. (1990). The machine that changed the world. New York: Rawson-Macmillan.

Wood, S. (1999, September). Getting the measure of the transformed high-performance organization. British Journal of Industrial Relations, 37(3), 391-417.

Wood, S., de Menezes, L., \& Lasaosa, A. (2001, May). High involvement management (Working Paper). Leicester, England: University of Leicester, Centre for Labour Market Studies.

Woodall, J. (2005, December). Beyond "good practice" in HRD: Balancing integrity, context and credibility. Keynote speech presented at the 4th Asian Conference of the Academy of HRD, Taipei, Taiwan.

World Bank. (2007). India: Data and statistics. Retrieved September 24, 2007, from www.worldbank.org.in

Wozniak, G. (1984). The adoption of interrelated innovations: a human capital approach. The Review of Economics and Statistics, 66(1), 70-79.

Wozniak, G. (1987). Human capital formation and early adoption of technology. Journal of Human Resources, 22(1), 101-112.

Yadapadithaya, P. S. (2001). Evaluating corporate training and development: an Indian experience, International Journal of Training and Development, 5 (4), 261-74.

Yang, B. (2004, May). Can adult learning theory provide a foundation for human resource development? Advances in Developing Human Resources, 6(2), 129-145.

Yin, R. K. (2003). Case study research: Design and methods. (3rd ed.). Thousand Oaks, CA: Sage.

Yorks, L. (2005). Nothing so practical as a good theory. Human Resource Development Review, 4(2), 111-113. 
Date:

\section{Dear Participant,}

Subject: Consent for conducting case study research.

Please permit me take this opportunity to introduce myself. I am currently Head of the Centre for Management at The Open Polytechnic of New Zealand and a PhD candidate at Victoria Management School, Victoria University of Wellington, New Zealand. In fulfilment of the requirements of this degree, I am undertaking a research project leading to a thesis, the title of which is: Factors Influencing the Provision of Enterprise Training: A Study of India's Information Technology (IT) Sector.

Regarding the specifics of this research project, developing human capital is vital to India's economic growth, especially for the IT sector of India, which has contributed significantly to the country's GDP, exports revenues, and employment growth. For this reason, the Government of India has accorded the sector a high priority and has set up a National Task Force looking at its human resource development needs to ensure that current growth rates are sustained and that the sector maintains its global competitiveness. In this regard, extant research suggests that improvements at a national level can only occur in the context of improved training provision at a firm level. With these factors in mind, this research project assesses the impact of factors thought to influence the provision of training at the enterprise level among firms in India's IT sector. The research project will also analyse the diversity and extent of training that is carried out among firms in India's IT sector, and the extent to which these firms rely on external or internal training providers for different types of training.

As the sampling frame for this research project, I am using NASSCOM's (National Association of Software and Service Companies) members' directory of IT and IT-enabled services companies. The nature of this study necessitates the use of questionnaires for collecting factual data and case studies for gaining an in-depth understanding of how training is organised and for determining the main factors that influence its provision. For these purposes, I, therefore, request formal consent from your organisation to enable me to conduct this research. 
Essentially, your organisation's participation in this will involve my being permitted access to human resource management and training professionals, project leaders, and a limited number project employees. In addition, this research will require some observation within your organisation and my having access to your organisation's human resource and training policies. It is envisaged that all of this will require that I have access to the aforementioned personnel and documents for a period of around 2 to 3 days.

Information derived using the aforementioned methods will form the basis of my research project and will be presented in aggregate form as a case study report. Any such information will remain confidential and measures have and will be taken to ensure that it will not be possible for you or your organisation to be identified individually from the reported results. All supporting documents will be destroyed after the completion of this research project. Moreover, please note that Victoria University requires that ethics approval be obtained for research involving human participants. To this end, this research project has received such approval from the University's Human Ethics Committee. Nevertheless, should any participant feel the need to withdraw from the project, they may do so, without question, at any time before 31 December 2006.

The final thesis will be submitted for marking to Victoria Management School and deposited in the University Library. It is intended that one or more articles based on the finding from this study will be submitted for publication in scholarly journals and/or presented at conferences/seminars. In addition, I will present a summary of the case study report to each respondent to confirm and verify accuracy of the relevant information contained therein. However, no person other than my supervisors, Dr Stephen Blumenfeld and Professor George Lafferty, and I will have access to the actual case study reports.

If you have any questions or would like to receive further information about the project, please contact me at Ashish.Malik@vuw.ac.nz or my supervisors, Dr Stephen Blumenfeld and Professor George Lafferty, at Stephen.Blumenfeld@vuw.ac.nz and George.Lafferty@vuw.ac.nz, respectively.

Ashish Malik

MA-PM\&IR (TISS, Mumbai)

$\mathrm{PhD}$ Candidate

Victoria Management School, Victoria University of Wellington, New Zealand

Phone: 00-64-4-913 5482 (W), 00-64-4-5682980 (H)

Enclosed: Consent Form 


\section{Victoria \\ Te Whare Wānanga \\ o te Ūpoko o te Ika a Māui

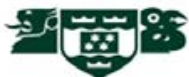

CONSENT FORM

APPENDIX-2

Title of project: Factors Influencing the Provision of Enterprise Training: A Study of India's Information Technology (IT) Sector.

I have been given sufficient information and explanation about this research project. I have had an opportunity to ask questions and have had them answered to my satisfaction. I understand that I may withdraw myself (or any information I have provided) from this project before 31 December 2006, without having to give reasons.

Further, I understand that any information I provide will be kept confidential to the researcher and his supervisors and that the published results will not use my name nor the organisation's name. I understand that the results of this research may be published or disseminated in professional or academic journals, seminars, conference proceedings, working paper, a summary report to case study participants, and as a thesis to be placed in the University's library.

Finally,

I would like to receive a summary report of the results of this research when it is completed.

I agree to take part in this research

Signed:

Name of participant:

Name of the organisation:

(Please print clearly) Date: 
1. Purpose and research questions

2. Key contributions of the project

3. The case study method

4. Organisation of this protocol

5. Case study questions (guiding notes)
a. Type of questions
b. Sources of evidence/data
c. Three key principles of data collection
d. Sampling strategy

6. Better understanding of the constructs
a. summary of questions
b. data sources

7. Relationship questions

8. Analysis plan and case study reports 


\section{CASE STUDY GUIDE}

\section{PURPOSE AND RESEARCH QUESTIONS (ex proposal document)}

- Chapters 1 and 3

\section{KEY CONTRIBUTIONS OF THE PROJECT}

Chapters 1 and 3

\section{THE CASE STUDY METHOD}

This study follows Yin's (2003) and Eisenhardt's (1989) case study research approaches. For details about case study research design (Yin, 2003) and theory building steps using case study research (Eisenhardt, 1989) refer to Chapter 4.

\section{ORGANISATION OF THIS PROTOCOL}

- Initial scheduling

\section{Sampling criteria: refer to Chapter 4}

- Contact case study organisations

-already contacted HR directors, Head - HR, CEOs of the above case study organisations and written consent received from all organisations

\begin{tabular}{|l|l|l|l|l|l|l|l|l|}
\hline Firms & $\begin{array}{l}\text { CEO/ } \\
\text { Country } \\
\text { Manager }\end{array}$ & $\begin{array}{l}\text { HR } \\
\text { Manager }\end{array}$ & $\begin{array}{l}\text { T\&D } \\
\text { Manager }\end{array}$ & $\begin{array}{l}\text { Project } \\
\text { Manager }\end{array}$ & $\begin{array}{l}\text { Quality } \\
\text { Manager }\end{array}$ & Employees & BD & Total \\
\hline A & & 2 & 1 & 3 & 2 & $\begin{array}{l}2 \text { (Informal } \\
\text { discussion) }\end{array}$ & & 10 \\
\hline B & & 1 & 2 & 2 & 1 & 2 & & 8 \\
\hline C & 1 & 1 & 1 & 2 & 1 & 6 & 1 & 13 \\
\hline D & $1 *$ & & & 2 & 1 & 6 & $1 *$ & 10 \\
\hline E & & 2 & & 2 & 1 & 1 & & 6 \\
\hline F & & 1 & 1 & 3 & 1 & & & 6 \\
\hline G & & 2 & 1 & 1 & 1 & 1 & & 6 \\
\hline H & & 2 & & 2 & 1 & 1 & & 6 \\
\hline I & 1 & 2 & & 3 & & 2 & & 8 \\
\hline J & $1 *$ & 1 & & 2 & & $\begin{array}{l}3 \text { (Informal } \\
\text { discussion) }\end{array}$ & $1 *$ & 7 \\
\hline Total & 4 & 14 & 6 & 22 & 9 & 24 & $1+(2 *)$ & 80 \\
\hline
\end{tabular}

Note: * are only counted once as the CEO/Country Manager was also the business development (BD) manager in Organisations $D$ and $J$

- Determining respondents/informants

-HR professionals

-Training managers

—Quality managers 
-Project team members

-Business development managers

-Project/process employees

\section{CASE STUDY QUESTIONS}

(to be used as prompts)

- Questions

- Semi-structured questions based on a priori constructs

— Additional questions to allow for flexibility for exploration of any emergent factors

- Sources of evidence/data

-Semi-structured Interviews

-Organisational documents and external publications about the organisation

-Direct observation

- Three key principles of data collection: See Chapter 4 for rationale

- Using multiple, not single, sources of evidence

- Creating a case study database

- Maintaining a chain of evidence

- Sampling strategy:

-Purposive maximum variation sampling

\section{BETTER UNDERSTANDING OF THE CONSTRUCTS}

- Use of a priori constructs

- Respondent's view of constructs

- Summary of questions

Note: These are prompts for semi-structured questions to be asked from one or more from the above list of possible informants/respondents.

- Data sources other than interview questions: annual report; induction manuals, website/ other published information, product catalogues/ project stories/case studies, business requirement specification documents for a project/ resource allocation/ team member composition/ target allocations/ charts etc.

Questions:

1. Background of the company: start by learning about the company's background.

2. Industry group: use literature categorisation or new; could emerge from background as well. 
3. Ownership: use extant literature categorisation or as described.

4. Nature of the main business activity: where available list activities by $\%$ of turnover.

5. Business strategy and main domains of business: industry verticals and domains; why certain verticals and domains only?

\section{Nature of competition}

- How would you describe the nature of competition for your products and services?

- Is it more from domestic or overseas competitors?

- How does the organisation gather information about its competitors?

- Does the organisation have an understanding of the strengths/weaknesses, long-term capabilities of its competitors

- Is the competition more for quality of services/products or price? Why?

- Is the market competing for certain skill sets?

\section{Enterprise size:}

- What is the total number of employees at this worksite as well as for the organisation as a whole?

\section{Nature of workforce:}

Work organisation: strategic/technical/administrative

- In terms of the key job roles performed, how would you describe the main categories of employees in your organisation?

- What is the percentage mix of this categorisation?

- In terms of a structure, how are projects teams/business units organised?

- What is the extent to which teams exercise work autonomy?

- Do they have freedom to carry out how to do a task?

\section{Workforce composition:}

- In terms of the nature of the employment, e.g. F/T P; P/T P; casual; contract, etc, how would you describe the current composition in your organisation?

- Why more full-time and low casual/contract/temps workers?

- In case of casual and part-time workers, do they have limited access to training opportunities? If not why?

9. Unionisation: little to non-existent

- If not, why are unions absent?

- What does the legislation say about it? Or is it a case of HR best practice?

- If present, what role do unions play in developing the capabilities of their staff members in terms of their on-the-job training and professional development

- What does the organisation perceive unions' role to be? 
10. Employee mobility (turnover):

- For the categories of staff identified before, what was the employee turnover rate for the last 12 months

- strategic

- technical

- admin \& support

- Other

- Common reasons for high/low employee turnover

- How does HR manage this high attrition rate?

- Is it becoming an industry norm to buy versus make?

- How do organisations retain/recruit people with new technology skills

\section{SHRM practices:}

\section{Integration of HR strategy with business strategy}

- Thinking about the organisation's current strategy, how would you describe its core strengths and direction for the future?

- How does it link with the organisation's people management strategy?

- Does the organisation analyse a list of competencies it needs for supporting its current and future strategy? If so, how?

- Do HR professionals get involved at the strategy formulation stage or only at the implementation stage? If the latter, why?

\section{Recruiting highly skilled employees}

- Are HR and line managers closely involved in the recruitment process?

- Is the focus of recruitment more on narrow range of skill sets that underpin a role or more on a broad set of competencies that underpin its existing/future strategy(ies) or both?

- How does the organisation manage recruiting for new or future skills sets? Do line managers who see potential in specific skill sets also feed such requirement to $H R$ ?

- Is the focus on recruiting at a high level of skills or are you focusing more on generic skill sets and looking at applicant's trainability?

- Do business development managers also have an input in the recruitment process?

- Do specific client requirements form the basis for input in the recruitment process?

- How do you go about the recruitment and selection process? Selection ratios 
Managing performance using the notion of core competencies

- What type of performance management system do you follow?

- Do you manage performance based on its core competencies?

- How closely is performance linked and assessed around an organisation's current strategy?

\section{Aligning an organisation's training to its business strategy}

- Does the organisation build its learning and development plans based on a pre-determined business strategy?

- How does the organisation ensure that alignment of training plans occurs at various levels

- Does a client's expectation indirectly influence the HR strategy and the training plans?

- How often do you have to align your training activities to your clients' requirements? If so, how do you go about doing that?

\section{Skills level}

- For the above composition of workforce described, would you classify their level of skill sets as high, medium, or low?

- Explore “make” versus "buy” decisions.

- Do employees have broad (generic) set of skill sets, such as - analytic ability, communication, problem solving, and supervision skills, or specialised (technical) skill sets, or is it a mix of both for different levels?

- Is trainability of employees a criterion when you are recruiting? If so, is it because of flexibility?

- $\quad$ Experience levels- high...medium ...low

\section{Volume}

- What is the extent of training provided as a \% of payroll

- Average number of training hours per employee?

- Any other training metrics

\section{Diversity}

o How is training organised in the organisation?

o What is the nature of training programmes provided by the organisation?

o Why is the focus more on certain types of training programmes?

o Do these training programmes reflect inadequacies in the education and formal training system or these are new skill requirements that are typical to the industry? 


\section{Externalisation}

- Does the organisation rely on external training providers? If so, why?

- What types of external training providers are used and their main focus on - behavioural or technical skills development?

- How often does training occur or is provided at clients' sites and by clients?

- Industry seminars?

- Does these programmes underpin job specific skills or broad and generic skill sets?

16. Type

- Describe the main categories for which training is provided

- Is the focus of these training programmes more on technical or generic skills?

\section{Formalisation}

- How does the organisation assess its training and learning needs?

- Is there a formal system for assessing/analysing training needs?

- How do you evaluate the training provided and in what terms?

- In such a dynamic environment, and given the continuously changing skill requirements, how does the organisation ensure skill sets it needs are current and relevant to the market and customers' needs?

- Are there any formal systems in place to identify and map the future competencies/skills needed?

\section{Learning Support}

- Does the organisation provide any financial and time support to employees for furthering their knowledge and skills? How does it work?

o What is the focus of such learning? - Firm and job specific or generic and broad based skills for personal development?

\section{Workplace change}

- What are the main changes that the organisation has witnessed in the last three years? And why? (Cover additional points such as downsizing, takeovers, new projects, processes services/products, introduction of team processes etc)

- Has the change affected all levels of workforce?(use the categories of workforce identified earlier)?

- How does the organisation communicates the change?

- How is it implemented? How often do these changes occur? 
- How does the organisation prepare its employees in managing the change process? Does it provide training to successfully implement a change initiative?

\section{Quality management approach}

- What is your organisation's overall approach to quality?

- Is investment in accredited quality programmes such as ...also seen as a marketing tool?

- How does investment in quality improve the organisation's business outcomes

- What is the main focus of various quality management initiatives- (explore individually customers- continuous improvement- improved productivityinfusing discipline in routines- looking at the organisation as a system)?

- How does the organisation ensure quality is implemented at various levels?

- How do employees ensure that customers get quality product/service?

- How does the organisation communicate the awareness across the organisation?

- In terms of its impact on training, which type of training is more associated with the adoption of quality management programmes?

\section{LO: commitment to learning; open-mindedness ; shared vision}

-What is your organisation's philosophy towards promoting a learning culture? How is it communicated?

-How does the organisation ensure that learning is shared?

-Are current knowledge, assumptions, and learning continuously challenged?

-Does the organisation critically reflect upon the information sensed and disseminated?

-How responsive is it in terms of its actions?

- Are strategies put in place to address any immediate issues such as errors, low service levels, new technologies?

\section{Team working: extent and autonomy}

- What percentage of your total workforce is organised around project/product teams. Are teams organised around specific skill sets?

- What is the frequency at which teams members rotate tasks within and between teams

- On an average, how many projects does a project team work on in any given year

- How do teams take decisions on issues such as organising work, targets, working with customer needs, skills requirements for a project, scheduling work etc

\section{Temporal and geographical dimension of training}

- How many projects/products/processes/ enhancements have been introduced 
in the last two years?

- How many of these projects/products/processes were new and how many were mere enhancements?

- How many of these projects/products/processes/ enhancements would you describe to be in the growth phase of the business cycle?

- How many of these products were from different countries /clients?

- How would you describe the process of product/ project development?

- How long does it take for a typical project to go live?

- What happens if the organisation does not have some of the skill sets for delivering/developing a project/product?

- Does the client provide any training support?

- Is the uptake of training, and developing competencies/skills, high in the initial phases of a project/product/process/ enhancements' business life cycle and if so why?

\section{Market orientation: information sensing; dissemination and responsiveness}

- How does your organisation go about collecting market and customer information about the products/ services in your organisation's portfolio?

- What is the focus of sensing this information- is it: market competition; customer needs; emerging technologies, new regulation or other...

- Where does the organisation collect this information from- existing customers, recruiting employees from competition, technology updates, etc

- How does the organisation ensure that the information sensed/collected is disseminated at various levels in the organisation?

- What sorts of communication strategies are employed for this?

- In what ways does the organisation respond to the information collected and disseminated - e.g. development of new products/ enhancements, improving processes, team meetings with project managers, HR and other functional policy changes, etc

\section{RELATIONSHIP QUESTIONS}

\section{Business activities (industry sector)}

- Does having a portfolio of numerous product/projects, as against one or few product/projects have an impact on (separately) diversity, volume and formalisation of training. If so, how?

\section{Ownership}


- Does ownership (use ownership details e.g. PVT Ltd/MNC / Indian Ltd etc) have an impact on training outcomes such as volume, diversity, externalisation, formalisation, externalisation and learning support? If so, why?

\section{Enterprise size}

- Do number of employees in the organisation impact on the volume of training? If so, why?

\section{Technological innovation}

- Does introduction of new products/ projects/ processes have an impact on the volume and diversity of training? If so, why? How does it impact the training infrastructure- formalisation / externalisation?

\section{QMS}

- Does the presence of a TQM philosophy have an impact on the volume and nature of training? If so, how?

- Does the presence of TQM philosophy require investment in certain types of technical and behavioural skills training? If so, what are these?

\section{Learning orientation}

- Does the presence of a learning culture have an impact on the volume and diversity of training? If so, why?

- Does the presence of a learning culture impact on the intelligence sensing, dissemination and responsiveness to a client's needs? If so, how?

\section{Market orientation}

- Does information sensing, dissemination and response have an impact on the number of new projects/products/processes in the organisation. If so, how many in the last 2 years?

- Does MO have an impact on learning culture and consequently introduction of new products? If so, how?

- Does MO influence the nature and extent of training provided? If so, how?

\section{Employee mobility}

- Does employee mobility have an impact on SHRM practices (recruitment, training, performance management)? If so, how?

- Does employee mobility have an impact on the types of training provided? If so, what are these?

- Does employee mobility have an impact on the volume and diversity of 
training?

\section{Skills level}

- Does level of skills have an impact on the volume and diversity of training? If so, how?

- Does level of skills have an impact on SHRM practices? If so, how?

\section{SHRM practices, workforce composition and workforce permanency}

- Does SHRM practices have an influence on workforce composition and permanency? If so, why?

- Does workforce permanency have an impact on the nature and extent of training?

- Does workforce composition have an impact on the type of training provided? If so, what are these types against different categories of employees?

\section{ANALYSIS PLAN AND CASE STUDY REPORTS}

-Details in Chapters 4, 5, and 6. 


\title{
PARTICIPATING CASE STUDY ORGANISATION'S FEEDBACK REQUEST FORM
}

\author{
Dear Case Study Participant,
}

In order to validate my understanding of various aspects that I studied about your organisation, I would welcome your reactions to the discussion and analysis presented in the case report. Your views, clarifications, opinions, corrections will provide me with more confidence in the casual figures and conclusions that have been presented in this report. Your input can show me where my explanations are partial or mistaken and need to be revised for accuracy, before the report is published and circulated to participating case study organisations. This is also part of the standard research practice to ensure validity and reliability of the analysis and conclusions presented (Miles \& Huberman, 1994).

Kindly note that I am requesting that you to consider the above analysis in light of that and the way things were in (Month, Year); the respondents whom I met; and what led up to that state of affairs.

1. Considering the text from 'Organisation X' case study report:

a. What are the main errors of fact that you see?

b. What are the main differences in interpretation that you have?

2. Considering the matrix and casual figures, and particularly looking at Tables and Figures, please answer the following questions:

a. I it an accurate version of the network; at least as it would have been in (Month, Year)? Kindly provide some narrative to record your reactions.

b. Are there any other key or mediating factors that I have missed and that you feel have an important impact on training provision? Please say a little about these in your response.

c. Looking at Figures, are any of these factors not important or of little significance? Kindly identify them with a ' $X$ ' and list any additional factors alongside or separately for each figure. For example, in the Figures, if there is an important factor '...' that seems to have been missed out, you may like to suggest, "It should be placed immediately before "size and complexity' and after 'quality' factors". 
d. Looking at Table 1 for any of the factors, have I provided an incorrect 'intensity' rating? Kindly correct this and provide any related explanation in the 'researcher's notes' column or separately.

e. Looking at Figures, do you think the direction of the arrows is accurate? If not please cross out the arrows and draw in new arrows. You may like to cross out the arrows with an 'X'. The track changes function in red is enabled and will help in identifying the new links.

$f$. Is the discussion at the end of each causal map accurate from your point of view? What other assumptions and, suggestions would you like to make to make it more accurate?

g. Would you like to draw a new map? If so, please feel free to do so here. You may have fewer or more factors in your revised/new map. But kindly keep in consideration the timing of the study, the respondents' views, and the researcher's interpretations of the latter. I would appreciate a practitioner's viewpoint.

Do you have any other concluding comments or suggestions to improve the accuracy of the draft? If so please write them down.

Yours sincerely

Ashish Malik

$\mathrm{PhD}$ Candidate

Victoria University of Wellington

New Zealand

ashish.malik@vuw.ac.nz 


\section{Appendix 5}

\section{Applying Six Sigma to a Simple Voice Process}

Presented here is an application of six sigma methodology to a simple voice process. Given the sensitive nature of information contained, preliminary data pertaining to agents' performance has been deleted to maintain anonymity and confidentiality of the respondents and case organisation. The data presented here is from one of the four ITeS/BPO organisations. Some key analyses are reproduced from one of the weekly reports. The report excerpts contain the following:

1. Quality sample audit sheet and performance report

2. Process defect Pareto

3. Stratification

4. Process dispositions

5. Process scorecard

6. Process wellness chart 
Quality Sample Audit Sheet and Performance Report

Executive:

er's Phone No:

Audit Category:

Week:

Agent ID: - Team Leader:

Call Type:

Manager:

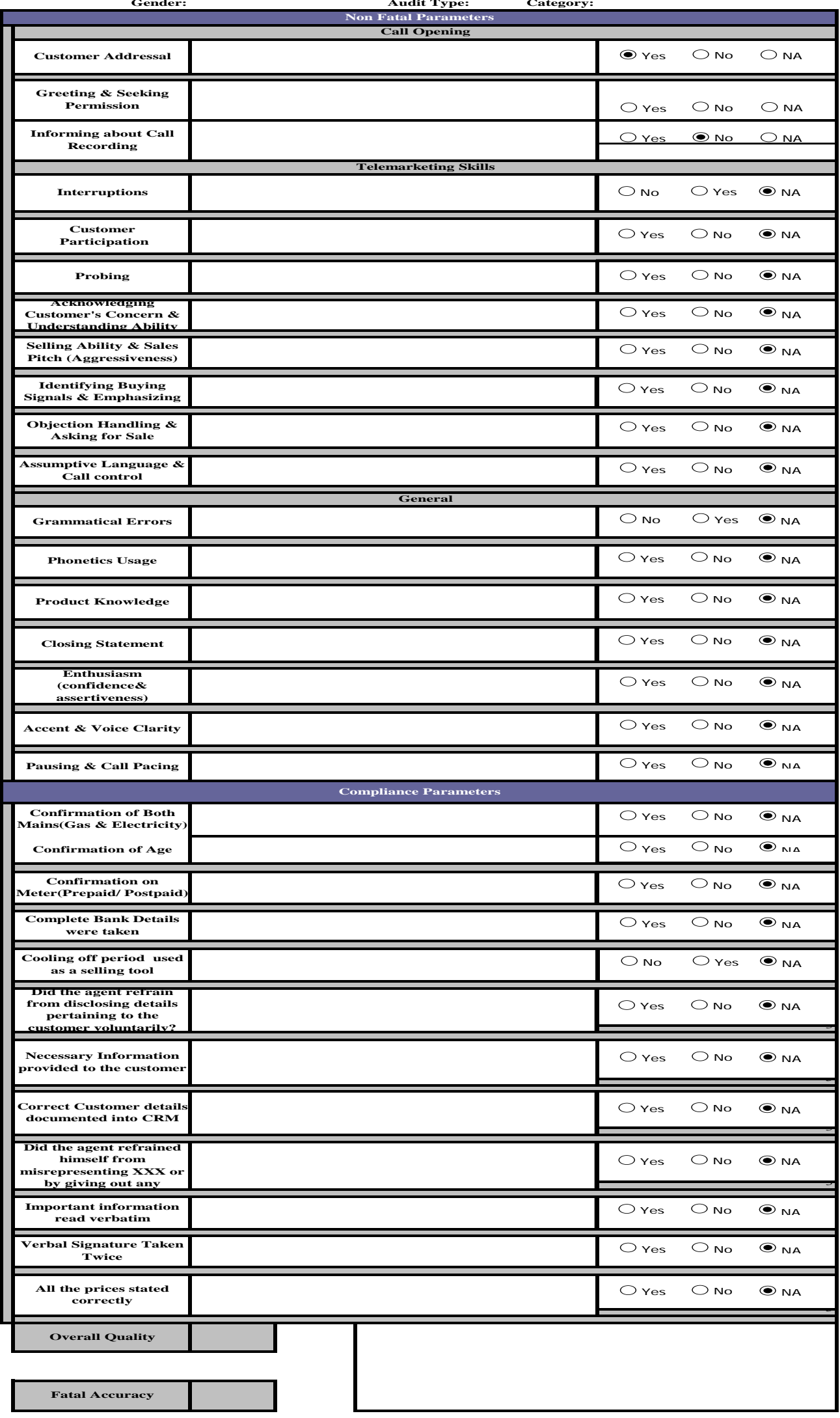




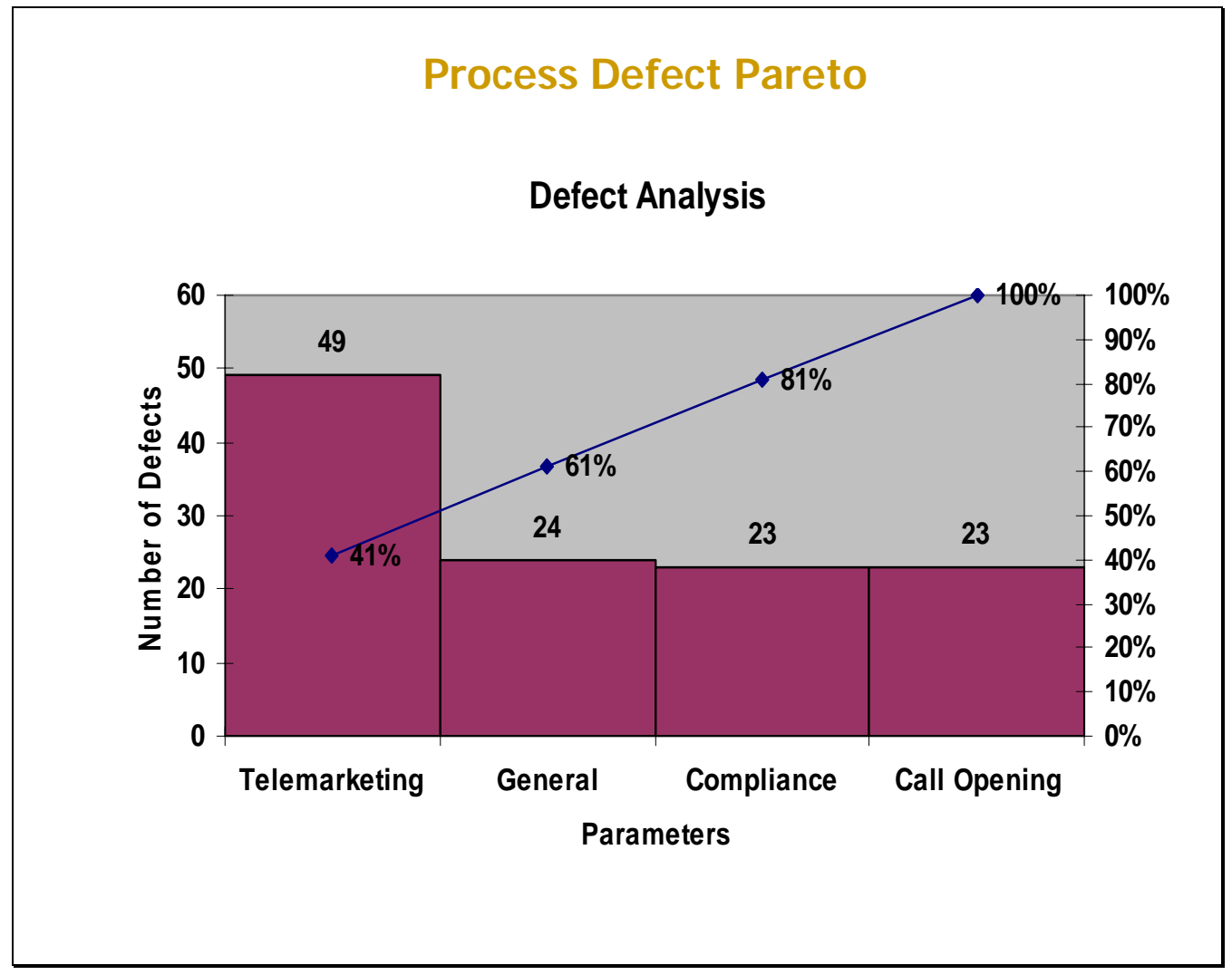




\section{Stratification}

\section{Telemarketing Defects}

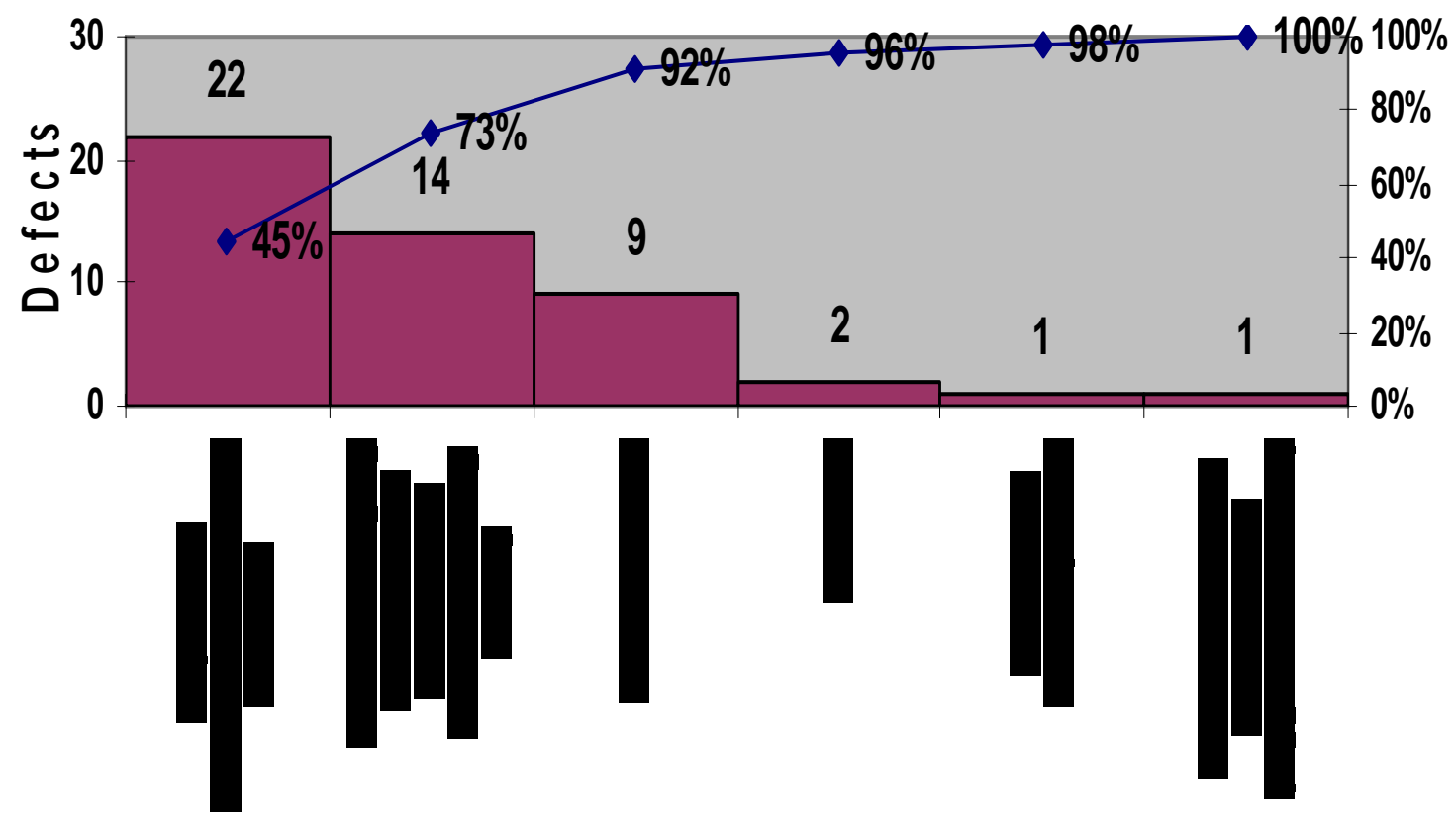

Parameters 


\section{Process Dispositions}

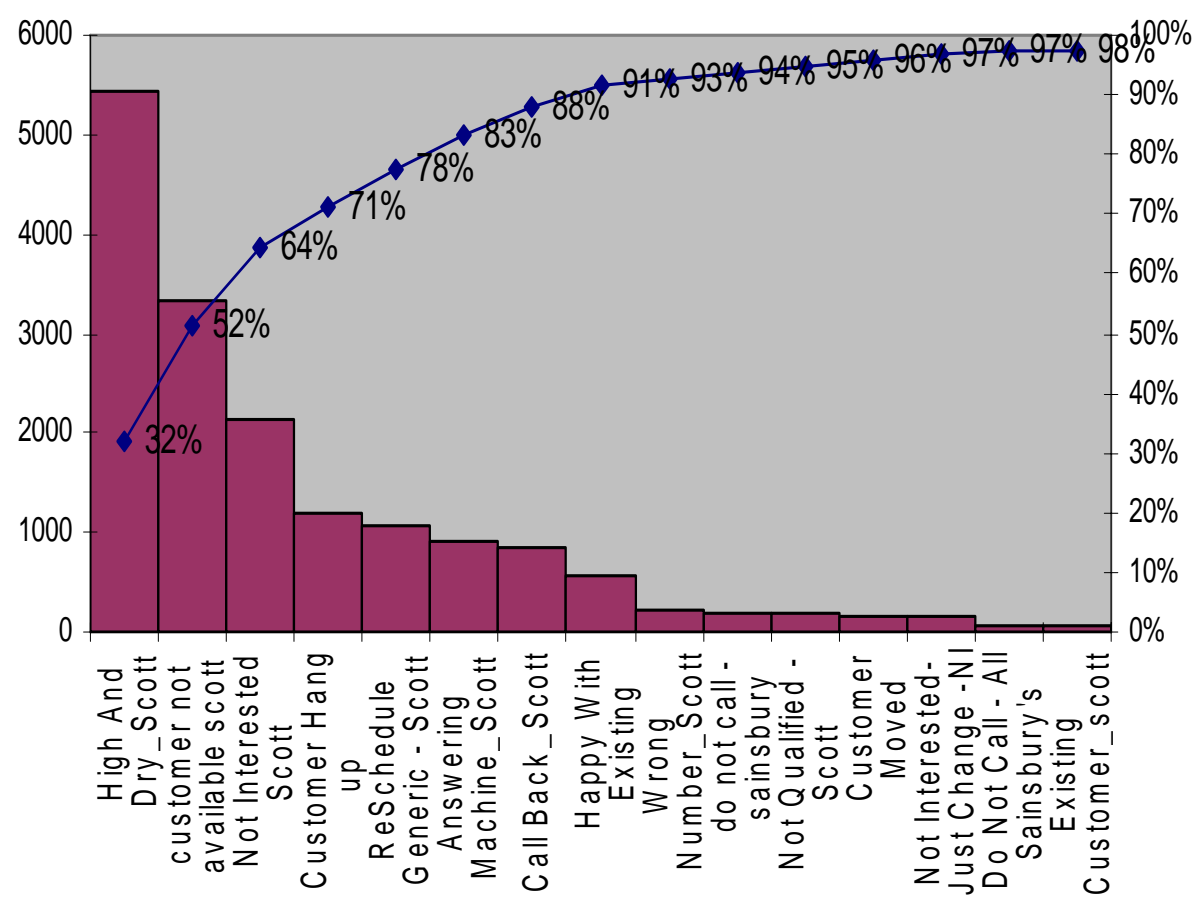




\section{Process Scorecard}

\begin{tabular}{|c|c|c|c|c|c|c|c|c|c|}
\hline S.No. & Required Metric & $\begin{array}{l}\text { Operational } \\
\text { Definition }\end{array}$ & $\begin{array}{c}\text { Reason for Selecting the } \\
\text { target }\end{array}$ & Direction of Good & $\begin{array}{l}\text { Target for } \\
\text { the Metric }\end{array}$ & Achieved & $\begin{array}{c}\text { Data captured } \\
\text { by }\end{array}$ & Percentage & SCORE \\
\hline 1 & Sales Per Hour & $\begin{array}{c}\text { Number of Sales } \\
\text { made by a agent }\end{array}$ & SLA & UP & 0.18 & 0.05 & MIS & $27.78 \%$ & 5 \\
\hline 2 & $\begin{array}{l}\text { Completes Per } \\
\text { Sale }\end{array}$ & $\begin{array}{c}\text { Agents } \\
\text { Completes Per } \\
\text { Sale }\end{array}$ & SLA & DOWN & 100 & 223.26 & MIS & $44.79 \%$ & 5 \\
\hline 3 & $\begin{array}{l}\text { Contacts Per } \\
\text { Hour }\end{array}$ & $\begin{array}{c}\text { Number of } \\
\text { contacts in a day }\end{array}$ & Internal Definition & UP & 15 & 10.42 & MIS & $69.47 \%$ & 3 \\
\hline 4 & Conversion & $\begin{array}{c}\text { Ratio of Sales to } \\
\text { Contacts }\end{array}$ & Internal Definition & UP & $1 \%$ & $0.49 \%$ & MIS & $49.00 \%$ & 5 \\
\hline 5 & Login Hours & $\begin{array}{c}\text { Production } \\
\text { Hours Clocked }\end{array}$ & SLA & UP & 1055 & 453.8 & MIS & $43.01 \%$ & 5 \\
\hline 6 & Talk Time & $\begin{array}{l}\text { Customer Facing } \\
\text { Time out of the } \\
\text { total login hours }\end{array}$ & Internal Definition & UP & $75 \%$ & $66.94 \%$ & MIS & $89.25 \%$ & 2 \\
\hline 7 & Wrap Time & $\begin{array}{c}\text { Time taken to fill } \\
\text { in the CRM or } \\
\text { assign } \\
\text { disposition }\end{array}$ & Internal Definition & DOWN & $10 \%$ & $16.92 \%$ & MIS & $59.10 \%$ & 3 \\
\hline 8 & Idle Time & \begin{tabular}{c|} 
Time between \\
calls
\end{tabular} & Internal Definition & DOWN & $15 \%$ & $16.12 \%$ & MIS & $93.05 \%$ & 2 \\
\hline
\end{tabular}




\section{Process Wellness Chart}

\begin{tabular}{|l|c|c|c|}
\hline & Category Significance & Performance Against Plans & Index Contribution \\
\hline Productivity & 40 & 27.78 & 11.112 \\
\hline Rate of Improvement & 25 & 0 & 0.00 \\
\hline Leads per sale & 15 & 44.79 & 6.72 \\
\hline Defect Rate & 10 & 12.28 & 1.23 \\
\hline Customer Satisfaction & 10 & 0 & 0.0 \\
\hline
\end{tabular}

\begin{tabular}{|l|c|}
\hline Process Wellness & 19.06 \\
\hline Process Defects Per Unit & 2.76 \\
\hline Process Defects Per Million Oppurtunities & 122,807 \\
\hline Process Sigma Level & 2.66 \\
\hline
\end{tabular}


